\title{
Induced pluripotent stem cell-derived cardiomyocytes as model for studying CPVT caused by mutations in RYR2
}

\section{Doctoral Thesis}

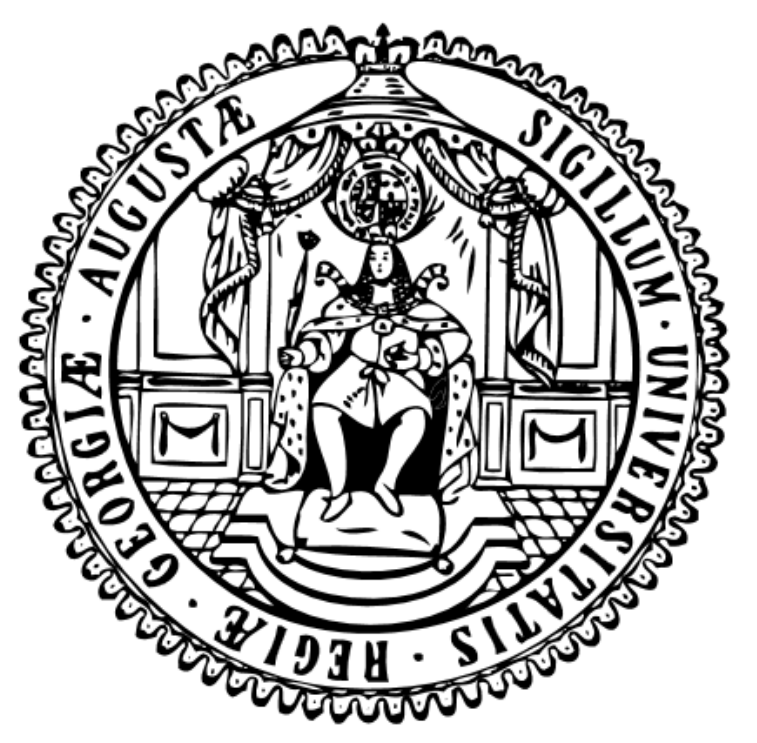

In partial fulfillment of the requirements for the degree

"Doctor rerum naturalium (Dr. rer. nat.)"

in the Molecular Medicine Study Program

at the Georg-August University Göttingen

\author{
submitted by \\ Sarah Henze
}

born in Göttingen, Germany 
Members of the Thesis Committee

First member of the thesis committee/ supervisor:

Prof. Dr. Kaomei Guan

Institute of Pharmacology and Toxicology

Technische Universität Dresden

Second member of the thesis committee:

Prof. Dr. Martin Oppermann

Institute for Cellular and Molecular Immunology

University Medical Center, Göttingen

Third member of the thesis committee:

Prof. Dr. Walter Stühmer

Department of Molecular Biology of Neuronal Signals

Max-Planck-Institute for Experimental Medicine, Göttingen

Date of Disputation: $29^{\text {th }}$ November 2016 


\section{AFFIDAVIT}

Here I declare that my doctoral thesis entitled "Induced pluripotent stem cell-derived cardiomyocytes as model for studying CPVT caused by mutations in RYR2" has been written independently with no other sources and aids than quoted.

Göttingen, October 2016 


\section{Table of Contents}

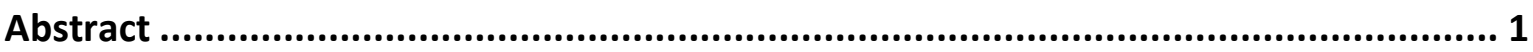

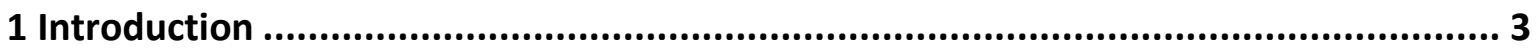

1.1 Catecholaminergic polymorphic ventricular tachycardia ........................................ 3

1.1.1 Clinical profile of catecholaminergic polymorphic ventricular tachycardia ......... 3

1.1.2 Ryanodine receptors in the context of CPVT ................................................ 4

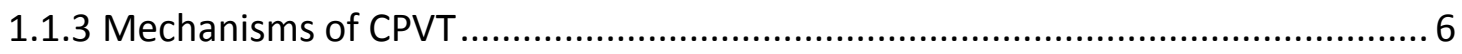

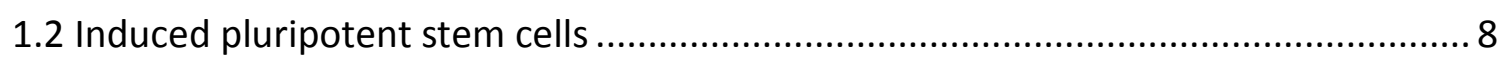

1.2.1 Modeling diseases with human pluripotent stem cells .................................. 10

1.2.2 Drug screening and cardiac toxicity tests using hiPSCs ................................... 13

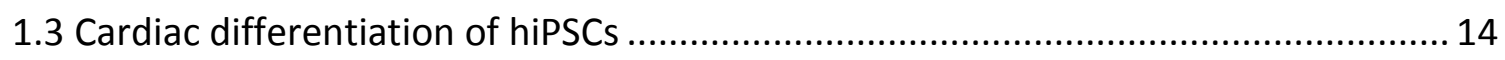

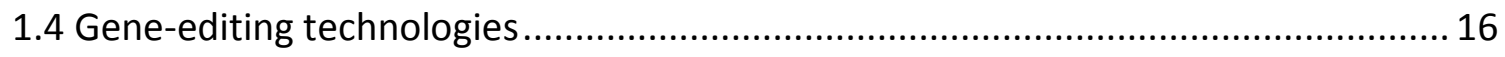

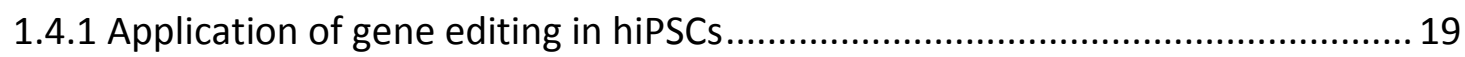

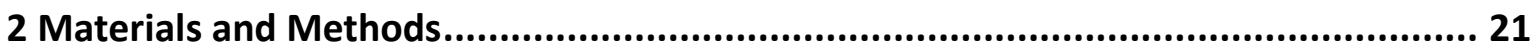

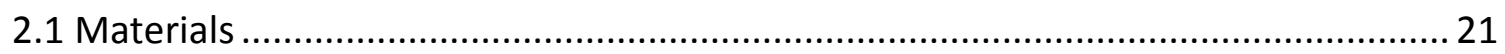

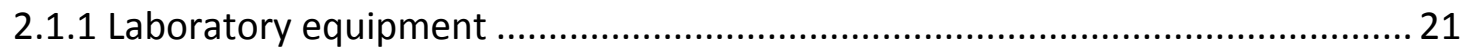

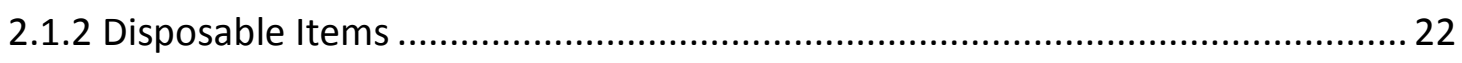

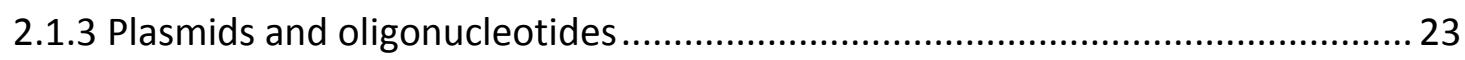

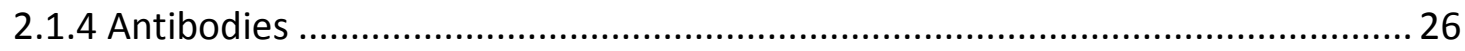

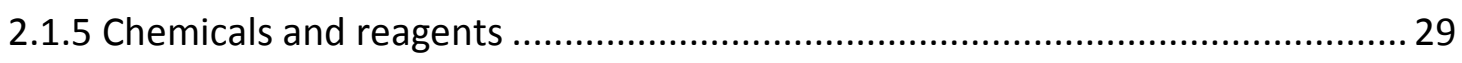

2.1.6 Solutions, buffers and bacteria material for molecular and protein analyses ... 31

2.1.7 Chemicals, solutions, and media for cell culture ............................................. 33

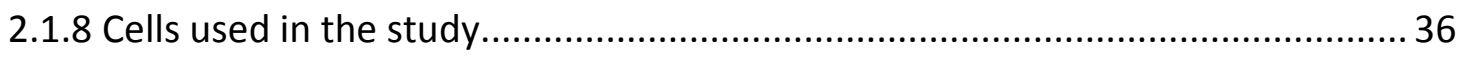

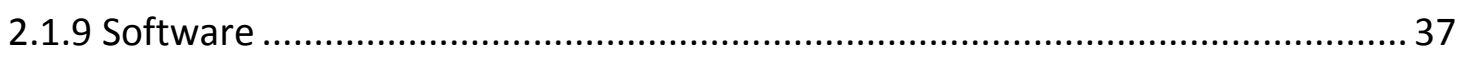

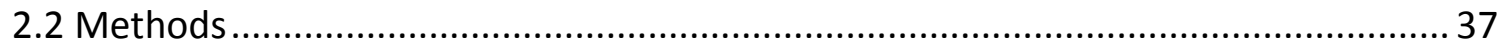

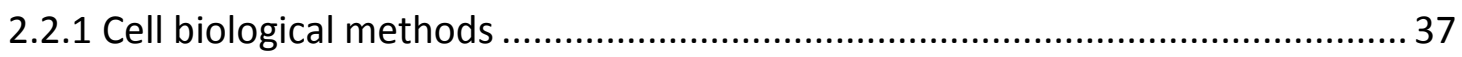

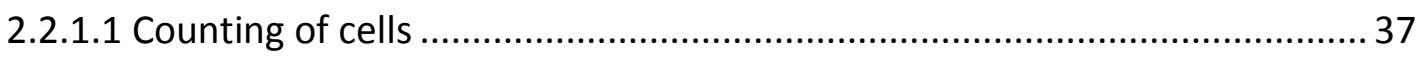

2.2.1.2 Cultivation and inactivation of mouse embryonic fibroblasts ..................... 37

2.2.1.3 Isolation and cultivation of human fibroblasts .......................................... 38

2.2.1.4 Generation of hiPSCs using CytoTune-iPS 2.0 Sendai reprogramming kit... 38

2.2.1.5 Cultivation of hiPSCs with feeder cells ..................................................... 39

2.2.1.6 Preparation of Geltrex-coated dishes and glass coverslips......................... 39 
2.2.1.7 Transfer of hiPSCs from feeder layer onto Geltrex-coated dish .................. 39

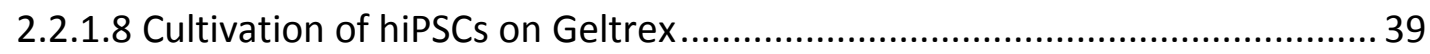

2.2.1.9 Spontaneous differentiation of hiPSCs in vitro ........................................ 40

2.2.1.10 Directed differentiation of hiPSCs into cardiomyocytes .......................... 40

2.2.1.11 Digestion and selection of iPSC-derived CMs........................................ 40



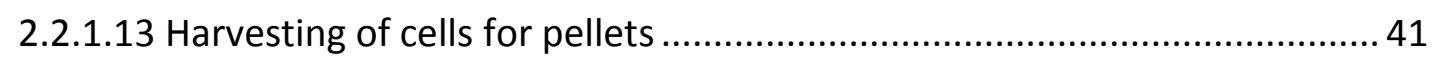

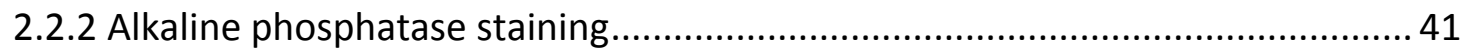

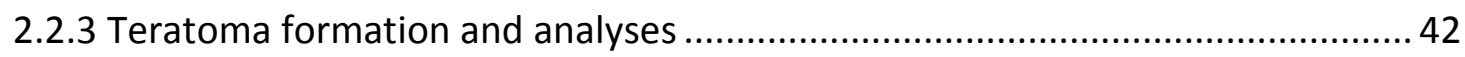





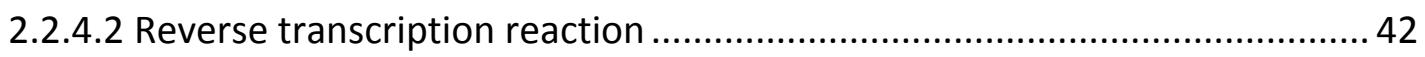

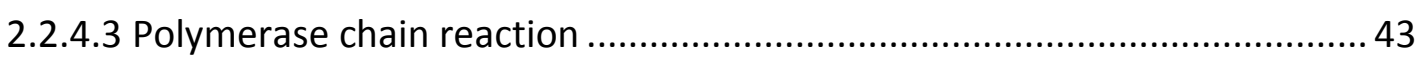

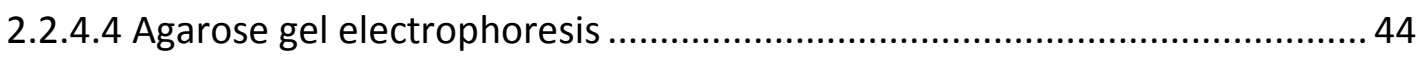

2.2.4.5 Purification of DNA fragments from PCR ............................................... 44

2.2.4.6 Heat-shock transformation of chemo-competent E. coli........................... 44

2.2.4.7 Plasmid purification from E. coli cultures................................................... 45

2.2.4.8 Genomic DNA isolation for DNA sequencing .......................................... 45

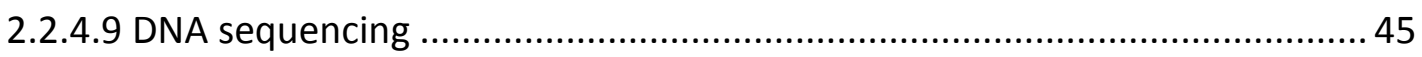

2.2.4.10 Allele-specific expression analysis of the $R Y R 2$ gene .............................. 45

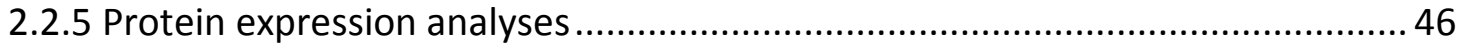

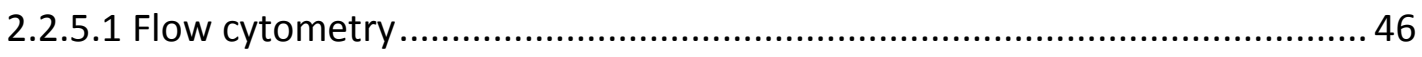

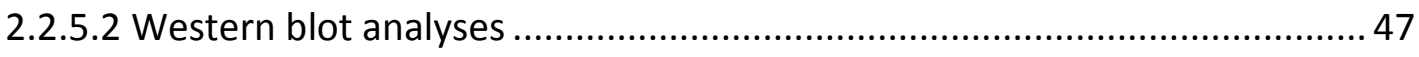

2.2.5.2.1 Preparation of cell lysates ................................................................. 47

2.2.5.2.2 SDS-polyacrylamide gel electrophoresis ........................................... 47

2.2.5.2.3 Protein transfer and detection ....................................................... 48

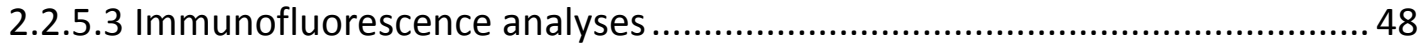

2.2.6 Generation of cell clones edited by CRISPR/Cas9 ......................................... 49

2.2.6.1 Transfection of HEK293T cells using calcium-phosphate ........................... 49

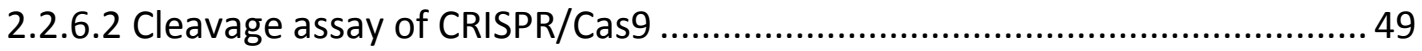

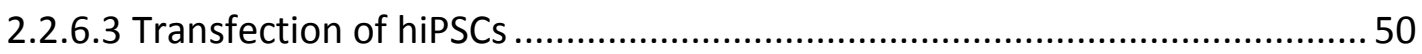

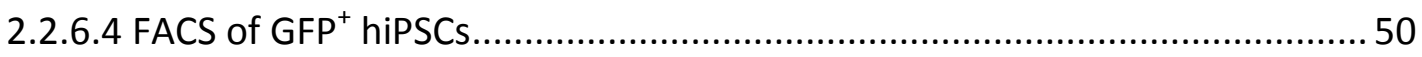

2.2.6.5 Expansion of cell clones edited by CRISPR/Cas9 …................................... 50 
2.2.6.6 Genomic DNA isolation and screening of cell clones edited by CRISPR/Cas9 51

2.2.6.7 Subcloning of sequenced CRISPR/Cas9 edited cell clones ......................... 51





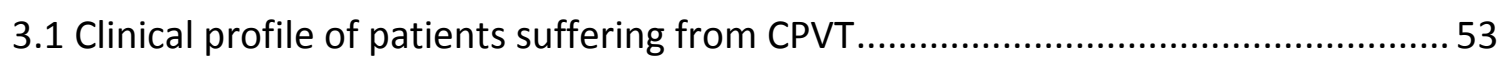

3.2 Generation of CPVT- hiPSCs and proof of their pluripotency.................................. 55

3.2.1 Generation of CPVT-hiPSCs from 3 different CPVT patients............................ 55

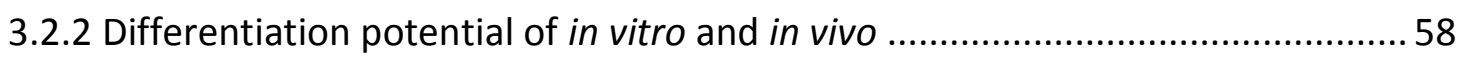

3.2.3 Verification of the RYR2 missense mutation in CPVT-hiPSCs .......................... 61

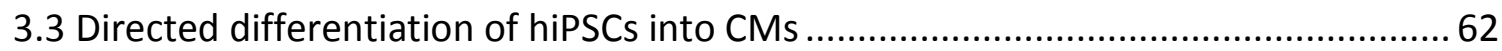

3.4 Role of heterogeneous RYR2 missense mutations in the pathogenesis of CPVT ...... 67

3.4.1 Allele-specific expression of RYR2 in CPVT-CMs on mRNA level .........................68

3.4.2 RYR2 expression and phosphorylation in CPVT-CMs .......................................69

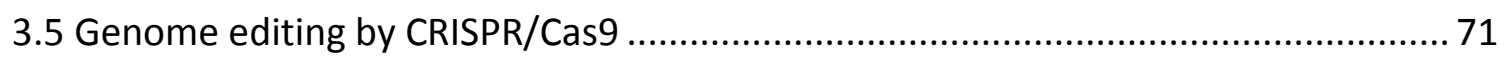



3.5.2 Generation of CRISPR/Cas9-targeted hiPSCs ................................................ 72

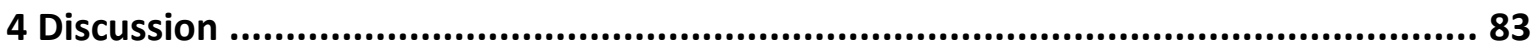

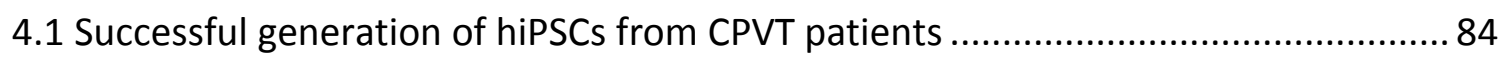

4.2 HiPSCs are able to differentiate directly into functional CMs ................................ 86

4.3 CPVT-CMs as model for studying disease-specific phenotypes in vitro ..................... 88

4.3.1 Pathophysiological comparison of clinical data and hiPSC-derived CPVT-CMs.. 89

4.3.2 Understanding the mechanism of CPVT disease .......................................... 92

4.3.3 Phosphorylation of Ser2808 and Ser2814 in CPVT-CMs.................................. 93

4.4 CRISPR/Cas9 as tool for efficient genome editing in hiPSCs .................................. 96

4.4.1 Generation of CRISPR/Cas9-edited CMs to study RYR2 function in hiPSCs........ 98

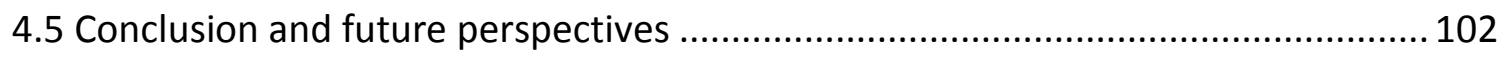

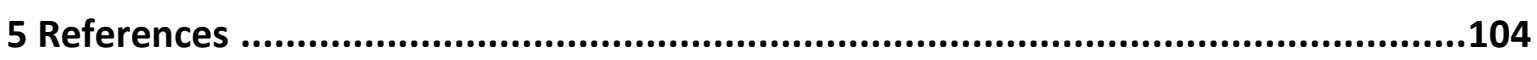

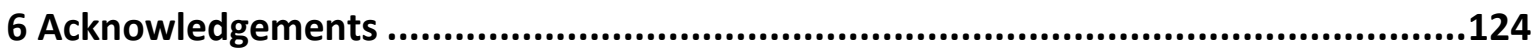

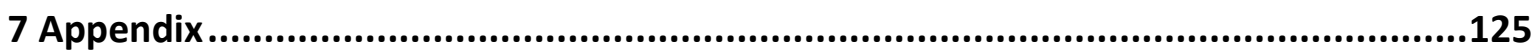

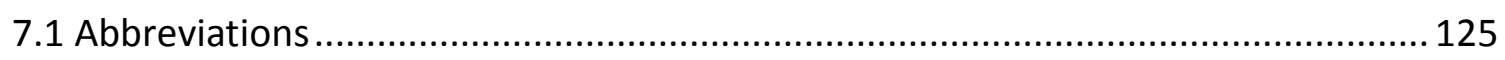

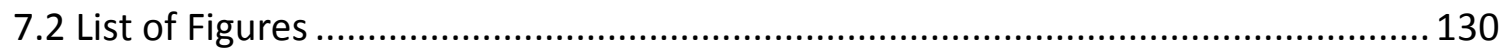

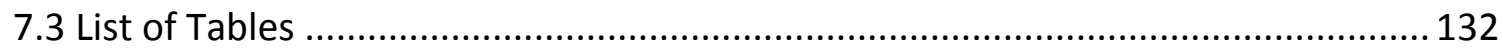









\section{Abstract}

Catecholaminergic polymorphic ventricular tachycardia (CPVT) is a severe hereditary cardiac ion-channel disease, which is caused by mutations in ryanodine receptor 2 (RYR2) or calsequestrin 2 (CASQ2). After $\beta$-adrenergic stimulation, these mutations lead to unwanted $\mathrm{Ca}^{2+}$ release from the sarcoplasmic reticulum (SR), which can cause life threatening arrhythmias, and sudden cardiac death. However, the underlying mechanisms how exactly point mutations in RYR2 induce arrhythmias have not been completely understood. Aim of this project was to establish in vitro disease models using human induced pluripotent stem cells (hiPSCs) as unlimited source for cardiomyocytes (CMs) and to study the molecular mechanism of CPVT.

In this study, hiPSCs were generated from three patients with CPVT symptoms caused by the heterozygous point mutations, A2254V and E4076K, in the gene RYR2. The generated hiPSCs showed pluripotency and were able to differentiate spontaneously into derivatives of all three germ layers. CPVT-hiPSCs and Ctrl-hiPSCs from healthy subjects were directly differentiated into functional ventricular $\mathrm{CMs}$ and tested for their purity by their expression of cardiac-specific marker proteins. Allele-specific sequencing showed balanced allelic expression of the disease-causing allele and healthy allele of $R Y R 2$ in CPVT-CMs. Western blot analysis showed that CPVT-CMs expressed similar levels of RYR2 in comparison to Ctrl-CMs. RYR2 phosphorylation (Ser2808, Ser2814) was not altered in CPVT-CMs carrying mutations R420W, A2254V and E4076K at basal or isoprenalinestimulated conditions when compared to Ctrl-CMs. This result refutes the hypothesis that stress induces hyperphosphorylation of RYR2 due to missense mutations in the RYR2 gene, at least not in CPVT-CMs with the mutations analyzed.

Furthermore, the CRISPR/Cas9 system was utilized for genome editing in hiPSCs. Homogeneous as well as heterogeneous CRISPR/Cas9-edited hiPSCs were generated and differentiated into CMs. CMs derived from RYR2 ${ }^{+/ \phi}-\mathrm{T} 42$-hiPSCs, which contain a heterogeneous deletion of $17 \mathrm{bps}$ in the $R Y R 2$ gene leading to a premature termination codon, manifested downregulated mRNA expression of the CPVT-causing allele. However, RYR2 expression in RYR2 ${ }^{+/ \varnothing}-\mathrm{T} 42-\mathrm{CMs}$ was not altered and comparable to CPVT-CMs. Moreover, RYR2 ${ }^{+/ \varnothing}-$ T42-CMs showed significantly reduced $\mathrm{Ca}^{2+}$ sparks, which were comparable to Ctrl-CMs, suggesting a potential healthy phenotype of CRISPR/Cas9-edited $\mathrm{RYR2}^{+/ \varnothing}$-T42-CMs. In contrast, CRISPR/Cas9-edited CMs with a homozygous deletion in RYR2 (RYR2 ${ }^{\varnothing / \varnothing}$-A3-CMs) showed normal RYR2 mRNA expression but no expression of the RYR2 protein. Interestingly, RYR2 ${ }^{\varnothing / \varnothing}-A 3-C M s$ showed nearly no SR $\mathrm{Ca}^{2+}$ leak due to the missing RYR2 protein. 
Taken together, the data demonstrate that patient-specific hiPSCs can be used to model CPVT. In CPVT-CMs with the mutations analyzed, the $\mathrm{Ca}^{2+}$ leak is not due to stressinduced hyperphosphorylation of RYR2. Knockdown of the CPVT-causing RYR2 allele can rescue, at least partially, the disease phenotype in CPVT-CMs. In addition, the data suggest that RYR2 is not required for the initiation of differentiation from hiPSCs into CMs. 


\section{Introduction}

\subsection{Catecholaminergic polymorphic ventricular tachycardia}

\subsubsection{Clinical profile of catecholaminergic polymorphic ventricular tachycardia}

Catecholaminergic polymorphic ventricular tachycardia (CPVT) is a severe inheritable cardiac disorder associated with life-threatening cardiac arrhythmias, syncope, seizures or sudden cardiac death in response to physical exercise or emotional stress (Coumel et al., 1978,; Jiang et al., 2002b). The prevalence of CPVT is estimated as 1:10,000 but its actual frequency in the general population is unknown (Faggioni and Knollmann, 2012). When the disease is untreated, approximately $30 \%$ of the affected subjects will develop symptoms before the age of 10 years and the majority of patients will have one or more arrhythmic patterns before age 40 (Leenhardt et al., 1995; Postma et al., 2005). Patients with CPVT have a structurally normal heart and normal 12-lead electrocardiography (ECG) recording at resting conditions (Leenhardt et al., 1995). Diagnosis of CPVT is demonstrated by ventricular arrhythmias such as bidirectional ventricular tachycardia (VT) during ECG recording with exercise testing. Due to the catecholamine induced phenotype of the disease, intravenous epinephrine infusion is used to aid the diagnosis of CPVT (Sy et al., 2011). In addition, screening for genetic mutations in cardiac genes associated with CPVT is often necessary for a correct diagnosis and an optimal therapy.

In the first step of therapy, CPVT patients should avoid exhausting exercise and stress. To reduce arrhythmic events, $\beta$-blockers such as nadolol, which is a non- selective $\beta$-blocker, or metoprolol and bisoprolol, cardioselective $\beta 1$-adenergic receptor blockers, are given as first-line treatment therapy. However, up to $40 \%$ of CPVT patients suffer again from cardiac events during long-lasting treatments with $\beta$-blockers (van der Werf et al., 2012). Administration of anti-arrhythmic drugs such as flecainide is the next step to suppress exercise-induced ventricular arrhythmias during therapy (van der Werf et al., 2011). Flecainide works by blocking the Nav1.5 sodium channel in the heart, slowing the upstroke of the cardiac action potential (AP) (Kvam et al., 1984; Ramos and O'Leary, 2004). Moreover, flecainide inhibits opening of potassium channels, especially the rapid component of the delayed rectifier $\mathrm{K}^{+}$current $\left(\mathrm{I}_{\mathrm{Kr}}\right)$ which prolongs the action potential duration (APD) in ventricular and atrial muscle fibers (Campbell and Vaughan, 1983). Recent data suggest that flecainide also blocks ryanodine receptor (RYR) opening, thereby reducing spontaneous sarcoplasmic reticulum (SR) calcium $\left(\mathrm{Ca}^{2+}\right)$ release, which potentially results in delayed afterdepolarization (DADs) and triggered activity (Watanabe et al., 2009; Liu et al., 2011). In new antiarrhythmic approaches Rycals such as JTV-519, 
S36 and S107 are used, which are 1,4-benzothiazepine derivatives that stabilize complexes of RYR and FK506-binding proteins (FKBP12.6) to prevent unwanted $\mathrm{Ca}^{2+}$ release (Yano et al., 2003; Wehrens et al., 2004a; Shan et al., 2012; Mohamed et al., 2015).

Most CPVT cases are predominantly linked to autosomal dominant point mutations in the RYR2 gene encoding the cardiac ryanodine receptor 2 (named CPVT1) or the autosomal recessive mutation in the gene encoding calsequestrin 2 (CASQ2) (named CPVT2) (Laitinen et al., 2003). Recently, mutations in the cardiac genes encoding calmodulin (CALM1) and triadin (TRDN) were also linked to CPVT (Nyegaard et al., 2012; Rooryck et al., 2015). Both autosomal dominant and autosomal recessive mutations alter the $\mathrm{Ca}^{2+}$ homeostasis of cardiomyocytes (CMs), thereby leading to ventricular arrhythmia.

\subsubsection{Ryanodine receptors in the context of CPVT}

RYRs are the largest known ion channels and mostly known for their important regulatory role in $\mathrm{Ca}^{2+}$ signaling that is essential for muscle contraction. The RYR protein is located in the transmembrane and forms homotetrameric assemblies with a total molecular mass of 2.2 MDa. Each monomer consists of $\sim 5,000$ amino acid (aa) residues from which approximately 4,300 aa forming the large cytosolic domain while $\sim 500$ aa are located in the membrane at the C-terminal region (Inui et al., 1987; Lai et al., 1988). In mammalians, RYRs are expressed in a wide variety of cell types, including CMs, skeletal muscle cells, neurons, exocrine cells, epithelial cells, lymphocytes, and many other cell types (Lanner et al., 2010). RYRs are named after the plant alkaloid ryanodine that binds with high affinity to the receptor and blocks its opening at high concentrations (Meissner, 1986). Three different isoforms (RYR1-3) have been identified, which share about $65 \%$ sequence identity. RYR1 is widely expressed in skeletal muscle (Takeshima et al., 1989). RYR2 is primarily found in the heart (Nakai et al., 1990), and RYR3 was originally identified in the brain in hippocampal neurons (Hakamata et al., 1992), although each isoform is found in lower amounts in several cell types. The cardiac RYR2 plays a central role in excitationcontraction coupling (ECC) of CMs. In this process, an action potential depolarizes the membrane and causes the release of $\mathrm{Ca}^{2+}$ ions from SR Ca ${ }^{2+}$ stores via RYR2, which then bind to the myofilaments to initiate contraction (systole). Following contraction, $\mathrm{Ca}^{2+}$ is pumped back into the SR by the $\mathrm{Ca}^{2+}$ ATPase SERCA (sarco/endoplasmic reticulum $\mathrm{Ca}^{2+}$ ATPase) inducing relaxation (diastole), which is essential for refilling the ventricles with blood (Fig. 1). In response to $\beta$-adrenergic stimulation during stress or exercise, one key feature of CPVT implies spontaneous aberrant efflux of $\mathrm{Ca}^{2+}$ escaping the SR via RYR2 during diastole. Increasing levels of intracellular $\mathrm{Ca}^{2+}$ gradually depolarize the membrane 
potential by activation of $\mathrm{Na}^{+} / \mathrm{Ca}^{2+}$ exchanger (NCX) and generate triggered early after depolarizations (EADs) or delayed after depolarizations (DADs). If the membrane potential reaches the threshold, a premature full AP is generated which in turn leads to severe arrhythmias. For the precise modulation of the channel gating, RYR2 associates with numerous binding proteins in the large cytosolic part including FKBP12.6, which stabilizes the receptor and calmodulin ( $\mathrm{CaM}$ ) and sorcin, which are calcium-binding proteins. On the luminal side, RYR2 binds to CASQ2, which is the major calcium storage protein in the $\mathrm{SR}$, as well as junctin and triadin, which interact with CASQ2, thereby, forming the combined SR calcium release unit (CRU) (Fig. 2). In vitro studies showed that RYR2 and CASQ2 mutations induce CRUs to open spontaneously, thereby, leading to intracellular $\mathrm{Ca}^{2+}$ overload, which in turn could trigger EADs (Paavola et al., 2007; Kujala et al., 2012) or DADs (Paavola et al., 2007).



Figure 1. $\mathrm{Ca}^{2+}$-induced $\mathrm{Ca}^{2+}$ release and triggered arrhythmias. When $\mathrm{CMs}$ are depolarized by an $\mathrm{AP}, \mathrm{Ca}^{2+}$ ions enter the cell through activated voltage-gated L-type $\mathrm{Ca}^{2+}$ channels (LTCC) located on the sarcolemma. A small amount of $\mathrm{Ca}^{2+}$ enters the SR and triggers a subsequent release of calcium that is stored in the SR through RYR2 by $\mathrm{Ca}^{2+}$-induced $\mathrm{Ca}^{2+}$ release (CICR, blue). $\mathrm{Ca}^{2+}$ released from the SR increases the intracellular $\mathrm{Ca}^{2+}$ concentration and free $\mathrm{Ca}^{2+}$ binds to troponin $\mathrm{C}$ that is attached to thin myofilaments and induces contraction. Relaxation is achieved by lowering the $\mathrm{Ca}^{2+}$ levels to diastolic values by SERCA for reuptake into the SR and by NCX that transports $\mathrm{Ca}^{2+}$ out of the cell. During stress situations, $\beta$-adrenergic signaling is activated leading to elevated levels of CAMP. CAMP functions as second messenger and activates protein kinase $A$ (PKA). Besides RYR2, PKA phosphorylates phospholamban (PLB) and LTCC, causing an increased $\mathrm{Ca}^{2+}$ uptake in the SR. When the threshold is passed, store overload-induced $\mathrm{Ca}^{2+}$ release occurs. Abnormal $\mathrm{Ca}^{2+}$ release can activate NCX which then may lead to DADs and arrhythmias (Modified from Priori and Chen, 2011). 


\subsubsection{Mechanisms of CPVT}

To date more than 150 CPVT-associated RYR2 mutations have been identified. Most RYR2 mutations were found as clusters in the four hot-spot regions called domain I/a (aa 44466), II/b (aa 2246-2534), III/c (aa 3778-4201), und IV/ d (aa 4497-4959) (Priori and Chen, 2011) (Fig. 2). However, exact mechanisms how different point mutations in $R Y R 2$ induce arrhythmias are not well defined. Several potential mechanisms have been proposed to explain why mutations in RYR2 channels lead to excessive release of $\mathrm{Ca}^{2+}$ during diastole, thereby causing life threatening arrhythmias. First, Jiang et al. proposed that CPVT mutations may lower the threshold for spontaneous $\mathrm{Ca}^{2+}$ release. After adrenergic stimulation, the concentration of SR free $\mathrm{Ca}^{2+}\left(\left[\mathrm{Ca}^{2+}\right] \mathrm{SR}\right)$ is physiologically increased. When $\left[\mathrm{Ca}^{2+}\right] \mathrm{SR}$ reaches a critical threshold, RYR2 opens and spontaneous $\mathrm{Ca}^{2+}$ release can happen even in the presence of normal channels. This process is called store overload induced $\mathrm{Ca}^{2+}$ release (SOICR) (Fernandez-Velasco et al., 2009).



Figure 2. Structure of RYR2 including hot-spot regions of mutations. The schematic illustration shows the 2D-structure of a single RYR2 monomer, including interaction sites with ancillary proteins, phosphorylation sites, and clusters of registered mutations called "hot-spot" domains. On the cytosolic part, RYR2 interacts with the protein phosphatases PP1 and PP2A, FKBP12.6 also called calstabin2, PKA, and CaM. The membrane proteins junctin and triadin are closely associated to the C-terminal part of RYR2 and regulate the $\mathrm{Ca}^{2+}$ levels by binding to CASQ2. Clusters of mutations in RYR2 are represented by violet lines and located at four "hot-spot" regions, which are named a/I (N-terminal), b/II (cytosolic), c/III (cytosolic), and d/IV (channel region) (Modified from Priori and Napolitano, 2005).

Moreover, CMs harboring RYR2 mutations present higher spontaneous $\mathrm{Ca}^{2+}$ release during diastole due to a dramatic increase in $\mathrm{Ca}^{2+}$ sensitivity of the RYR2 (Jiang et al., 2004; Fernandez-Velasco et al., 2009). More recently, studies demonstrated that RYR2 acts as sensor and is responsible for $\mathrm{Ca}^{2+}$ storage of the luminal $\mathrm{Ca}^{2+}$ and SOICR. 
Furthermore, defective RYR2 inter-domain interactions were reported to trigger CPVT episodes. In this scheme, mutated RYR2 channels are believed to get hyperactive due to defective inter-domain interactions that "zip" N-terminal and central regions of RYR2, thereby causing diastolic $\mathrm{Ca}^{2+}$ leakage (Ikemoto and Yamamoto, 2002; Tateishi et al., 2009).

One important regulator of RYR2 is the $12 \mathrm{kDa}$ protein FKBP12.6 which binds to RYR2, thereby stabilizing the closed state during diastole to prevent $\mathrm{Ca}^{2+}$ release from the SR. Former studies supposed that mutant RYR2 channels have a decreased binding affinity to FKBP12.6 which might cause triggered activity of RYR2 (Marx et al., 2000; Wehrens et al., 2004a). However, this mechanism seems to be mutation-specific because other studies showed normal interaction of mutated RYR2 with FKBP12.6 (George et al., 2003). Protein kinases such as protein kinase $\mathrm{A}$ (PKA) and $\mathrm{Ca}^{2+} / \mathrm{calmodulin}^{2}$-dependent protein kinase II (CaMKII) play important roles in the calcium homeostasis by phosphorylating $\mathrm{Ca}^{2+}$ regulatory proteins such as phospholamban (PLB) and RYR2. Alterations of phosphorylation could alter the function of RYR2 channel leading to cardiac diseases, heart failure, and arrhythmias by enhanced $\mathrm{Ca}^{2+}$ leak. For this reason, another hypothesis supposed that hyperphosphorylation and loss of phosphatases is responsible for the increased $\mathrm{Ca}^{2+}$ leak. Increased phosphorylation could lead to dissociation of FKBP12.6 from RYR2, thereby causing hyperactive leaky channels (Marx et al., 2000; Wehrens et al., 2006; Shan et al., 2010). Currently, RYR2 has three well-defined phosphorylation sites, Ser2030, Ser2808, and Ser2814 (Ser2809 and Ser2815 in rabbit) although there might be others (Rodriguez et al., 2003; Xiao et al., 2005). The degree of basal phosphorylation depends on a dynamic balance between multiple protein kinases and phosphatases (Heijman et al., 2013). Ser2808 and Ser2030 are mainly phosphorylated by PKA (Marx et al., 2000; Xiao et al., 2006). CaMKII regulates predominantly phosphorylation of RYR2 residue Ser2814 (Wehrens et al., 2004b). It is also likely that other serine/threonine kinases can phosphorylate RYR2 (Ather et al., 2013; Marx and Marks, 2013). Protein phosphatases PP1 and PP2A associate with RYR2 via the anchoring proteins spinophilin and PR130, respectively, thereby regulating dephosphorylation of RYR2 (Marx et al., 2001). Current studies had controversial results regarding the phosphorylation status of distinct sites in different disease model systems (mouse, rabbit, human) and the role of RYR2 phosphorylation and dephosphorylation seems to be very complex.

The majority of CPVT-linked RYR2 mutations generate hyperactive leaky channels, also called "gain-of-function", but hypoactive channels with decreased open probabilities were also reported and named "loss-of-function". Examples for RYR2 mutations causing gain-of-function include among several others: R176Q and L433P located in the Nterminal region; S2246L and R2474S located in the central region; as well as S4153R, 
Q4201R and 14867M located in the C-terminal region (Jiang et al., 2005; Zhabyeyev et al., 2013). HEK293 cells expressing recombinant RYR2, including these mutations, displayed enhanced propensity for spontaneous $\mathrm{Ca}^{2+}$ release and reduced threshold for SOICR, which are common features of CPVT-linked gain-of-function RYR2 mutations (Jiang et al., 2005).

In contrast, heterozygous Ryr2 mutations A4860G and 14855M are described as loss-offunction mutation in mice. Ventricular myocytes isolated from mice carrying the Ryr2 mutation A4860G have constitutively lower $\mathrm{Ca}^{2+}$ release than WT mice, and undergo apparently random episodes of prolonged systolic $\mathrm{Ca}^{2+}$ release upon $\beta$-adrenergic stimulation, which give rise to EADs (Jiang et al., 2007; Zhao et al., 2015). HEK293 cells expressing recombinant RYR2-14855M exhibited inhibited caffeine-induced $\mathrm{Ca}^{2+}$ release (Roston et al., 2016). The closely located Ryr2 mutation E4782Q completely protects against SOICR-induced arrhythmias in a CPVT mouse model harboring the disease-causing Ryr2 mutation R4496C (Chen et al., 2014).

\subsection{Induced pluripotent stem cells}

The human induced pluripotent stem cell (hiPSC) technology provides a powerful tool in which somatic cells are reprogrammed with pluripotent associated transcription factors into a pluripotent state with unlimited proliferation and differentiation ability (Takahashi et al., 2007) (Fig. 3). The first set of reprogramming factors was composed of OCT4 (octamer-binding transcription factor 4), SOX2 (sex determining region $Y$ box 2), KLF4 (Krüppel-like factor 4) and C-MYC (v-Myc myelocytomatosis avian viral oncogene homolog) and introduced by retroviral and lentiviral transduction methods (Takahashi et al., 2007). Other combinations such as OCT4, SOX2, NANOG and LIN28 were also sufficient to induce pluripotency (Yu et al., 2007). HiPSCs have quite similar properties to human embryonic stem cells (hESCs) in terms of morphology, proliferation ability, surface marker, gene expression, promoter activities and telomerase activity (Takahashi et al., 2007). Like hESCs, hiPSCs can differentiate in vitro into derivatives of all three primary germ layers (ectoderm, mesoderm, and endoderm) and form teratoma following subcutaneous injection into immunodeficient mice (Takahashi et al., 2007). In contrast to hESCs, which are derived from the inner cell mass of developing embryos, hiPSCs do not give rise to ethical concerns and have the potential for future clinical transplantations. Until now, several reprogramming techniques were developed including viral-mediated transgene overexpression and viral-independent approaches such as plasmids, episomal, protein or mRNA-mediated overexpression (Stadtfeld et al., 2008; Kim et al., 2009; Jia et al., 2010; Warren et al., 2010). 




Figure 3. Generation of patient-specific hiPSCs for disease modeling and drug screening. HiPSCs can be generated from patient-specific somatic cells and then transformed into specialized cells by directed differentiation. In vitro differentiated human cells provide an easy accessible source for applications in disease modeling to study the molecular phenotypes of diseases, or for screening assays that enable drug discovery as well as toxicity tests for various somatic sells. These applications can enrich the knowledge of new drugs to improve therapies of serious diseases (Bellin et al., 2012).

High throughput and efficient generation of hiPSCs can be achieved by the usage of the single polycistronic lentiviral vector STEMCCA that compromises the four reprogramming factors in a single plasmid (Sommer et al., 2009). However, retroviruses and lentiviruses integrate into the cell genome and can interfere with the coding sequence, thus affecting transcription and protein function. Another serious problem is the gene set itself, which is used for reprogramming, because it could induce spontaneous tumor formation in case of future clinical administration. The expression of OCT4, SOX2, KLF4, and C-MYC is associated with the development of multiple tumors known in oncogenetics (Okita et al., 2007; Ben-Porath et al., 2008). Expression of C-MYC is observed in $70 \%$ of human cancers (Kuttler and Mai, 2006). For this reason, it is not possible to use hiPSCs generated with lenti- or retroviruses in future clinical applications for cell replacement therapies.

Currently, integration-free techniques for reprogramming such as Sendai virus (SeV) are commonly preferred. The F-deficient SeV is a non-transmissible, negative sense, singlestranded RNA virus, which replicates in the cytoplasm of targeted cells without integrating into the host genome (Fusaki et al., 2009a). Nevertheless, transductions with $\mathrm{SeV}$ are expensive and require more biosafety standards compared to non-viral methods. Moreover, viral material persists for longer periods in cell culture. The latest integrationfree as well as cost-effective method is the CoMiP vector system that compromises all 4 reprogramming factors in one plasmid and overcomes the problem of viral material 
(Diecke et al., 2015). Various chemical substances, such as valproic acid, which is a histone acetylase inhibitor, were applied to enhance reprogramming efficiency (Huangfu et al., 2008). In addition, recent studies showed that supplementary nutrition factors, such as sodium butyrate and ascorbic acid, contribute to reprogramming efficiency and hiPSC quality (Esteban et al., 2010; Mali et al., 2010). With the use of different methods, various cell types were effectively reprogrammed, including mesenchymal stem cells (MSCs), fibroblasts, peripheral blood mononuclear cells (PBMCs), and keratinocytes (Churko et al., 2013). Reprogramming of MSCs into hiPSCs is more efficient compared to the usage of fibroblasts or keratinocytes (Streckfuss-Bomeke et al., 2013). However, it is more difficult to obtain MSCs from patients compared to other cell sources.

In the first years of hiPSC research, culture media for derivation and expansion of hiPSCs was based on culture conditions for hESCs. These conditions include the cultivation of hiPSCs on murine-derived feeder cells in combination with medium containing knockout serum replacement. Preparation of feeder cells requires significant time and effort, and they are cultured in fetal bovine serum (FBS)-containing medium, which has often large batch-to-batch variations. In the last years, feeder-free culture systems for hESCs and hiPSCs were developed to prevent spontaneous and unwanted differentiation during cultivation on feeder cells, thus improving the quality of conventional culture systems. Feeder cells were replaced by various matrices such as Matrigel, CELLstart, Geltrex and recombinant proteins such as vitronectin and laminin as well as synthetic polymers (Rodin et al., 2010; Chen et al., 2011b; Stover and Schwartz, 2011). Chemically defined media are nowadays commercially available, including in particular TeSR2, Essential 8 (E8) medium, and NutriStem (Chen et al., 2014).

\subsubsection{Modeling diseases with human pluripotent stem cells}

Reprogramming of cells obtained from patients into hiPSCs opens many possibilities for disease modeling. Patient-specific hiPSCs retain the genetic characteristics of their donors which enable the study of genotype-dependent disorders at cellular and molecular levels. In the last years, hiPSCs as well as hESCs were used to investigate various severe genetic diseases and brought new cellular and molecular mechanisms as well as genetic phenotypes forward.

Genetic diseases can be divided into different types such as monogenetic, chromosomal, and complex diseases. Monogenetic diseases develop from mutations in single genes, whereas chromosomal diseases arise from either loss or addition of whole chromosomes or parts of it. Complex disorders originate from alterations in different genes or have an 
unknown genetic cause. Diseases from all three types were successfully modeled using hiPSCs. Monogenetic diseases include among others the Lesh-Nyhan disease and the fragile $X$ syndrome (Urbach et al., 2004; Eiges et al., 2007). Chromosomal diseases include modeling of the Turner and Down syndrome and examples for complex disorders are autism spectrum disorder and schizophrenia (Urbach and Benvenisty, 2009; Brennand et al., 2011; DeRosa et al., 2012; Briggs et al., 2013). Especially for early onset diseases, which occur during fetal development and childhood, hiPSC models are well suited because several differentiated cell types from hiPSCs resemble more an embryonic rather than an adult phenotype. Modeling of late onset diseases, such as Alzheimer disease, is more difficult and cellular maturation as well as ageing must be enhanced using sophisticated differentiation protocols or longer culture periods (Studer et al., 2015).

In the last years, hiPSC technology gained strong attraction for cardiovascular medicine due to limited numbers of well-suited disease models. CMs isolated from patient biopsies can be used as tools but cardiac cells are difficult to obtain in adequate quantities and cannot be kept in culture for prolonged time periods or expanded in vitro. Rodent models carrying mutations causing cardiac diseases are actively used as alternative model to study mechanisms of diseases (Salama and London, 2007; Zaragoza et al., 2011). However, these models have some restrictions due to differences in cardiovascular physiology between rodents and humans, such as distinct physiological beating rates ( 600 beats per minute in mice), repolarization phase of action potentials, and ion channel composition. Due to the same genetic background in rodent models, their use is limited for disease models because animal models can often not explain why patients carrying similar disease-causing mutations vary remarkable in their phenotype severity and drug response.

These obstacles can be overcome by modeling heart diseases with patient-derived hiPSCs that are directly differentiated into large quantities of CMs. In 2010, the LEOPARD syndrome was modeled as first heart disease using patient-derived hiPSCs which were differentiated into CMs (Carvajal-Vergara et al., 2010). The following years, several inherited cardiac arrhythmogenic disorders, such as different subtypes of the long-QT syndrome (LQTS), Brugada syndrome and CPVT, were analyzed regarding their disease phenotypes and drug rescue (Moretti et al., 2010; Itzhaki et al., 2011a; Itzhaki et al., 2012; Kujala et al., 2012; Caspi et al., 2013; Cerrone et al., 2014). To investigate the phenotype of these channelopathies, electrical properties of cardiomyocytes can be measured by multielectrode arrays (MEAs) and patch clamp recordings and compared to CMs derived from healthy subjects. Disorders that cause defective structures, contractility and survival including dilated cardiomyopathy, hypertrophic cardiomyopathy, Barth syndrome and 
arrhythmogenic right ventricular dysplasia (ARVD), have been also modeled (Sun et al., 2012; Caspi et al., 2013; Dudek et al., 2013; Lan et al., 2013).

Besides the advantages of hiPSC-based disease models, hiPSC-derived CMs are often criticized due to their immature phenotype resembling embryonic CMs when compared with native $\mathrm{CMs}$ in regards to molecular, structural, metabolic, and functional aspects. HiPSC- and hESC-derived CMs cultured in 2D-culture are much smaller in size, round or multangular in shape, and have often a more flattened structure when compared with adult CMs. Proportional to their smaller cell surface area, hiPSC-derived CMs have lower membrane capacitance with 49-68 $\pm 6.89 \mathrm{pF}$ (Stauske et al. unpublished data) in comparison to adult ventricular CMs that have approximately 150 pF (Drouin et al., 1995; Zhu et al., 2010). In addition, ultrastructure studies revealed that hiPSC-derived CMs lack a fully developed transverse tubule system and have disorganized shorter sarcomeres (Lieu et al., 2009; Gherghiceanu et al., 2011). Electrophysiological differences include spontaneous contraction, less negative maximum diastolic membrane potential, low maximal upstroke velocity and prolonged APD compared with adult CMs that do not contract spontaneously. Differences were also found in gene expression and cell function. For example, hiPSC-derived CMs have lower amounts of mitochondria which are located in the perinuclear region while adult CMs have randomly distributed mitochondria which occupy about $20-40 \%$ of the cell volume (Zhang et al., 2015). Transcriptome analysis reported that gene expression of hiPSC-derived CMs was similar to the first trisemester of the human fetal heart (van den Berg et al., 2015). Expression of MYH6 ( $\alpha-\mathrm{MHC}$ ), TNNT1 (fetal SsTNI) and titin isoform N2BA predominates in hiPSC-derived CMs while MYH7 ( $\beta$ MHC), TNNI3 (cTNI) and titin isoform N2B are expressed mainly in adult CMs (Denning et al., 2016).

Prolonged culturing periods of hiPSC-derived CMs (80-120 days) demonstrated electrophysiological phenotype maturation with more hyperpolarized diastolic potential, faster upstroke velocity, increased connexin 43 (CX43) expression, and higher rates of calcium release and uptake (Lundy et al., 2013). Adding of supplements such as triiodothyronine, which is important for cardiac development, during prolonged culture periods increased the maturation of $\mathrm{CMs}$ including enhanced oxygen consumption and force development (Yang et al., 2014). In addition, electrical stimulation with $1 \mathrm{~Hz}$ for 14 days mimicked fetal heart development and promoted the maturation of hiPSC-derived $\mathrm{CMs}$. The increased maturation status was recognized by the upregulation of the potassium channel KIR2.1 and sacromeric proteins, the maturation of the AP shape, the increased number of quiescent cells and mature $\mathrm{Ca}^{2+}$ handling properties (Lieu et al., 2013). In another study, maturation was enhanced when fetal CMs were cultured in a 
3D-matrix and subjected to mechanical stress (Zimmermann et al., 2002; Soong et al., 2012).

Nevertheless, complex mechanisms of terminal differentiation are far from being fully understood and the achieved increased maturation of hiPSC-CMs is still more similar to the fetal-like phenotype, than to adult CMs. However, several studies of cardiac disease modeling showed that hiPSC-derived CMs work fine as additional tools for human models, but cannot replace completely in vivo studies using animal models. As mentioned before, animal models have also large limitations when they are used for studying human phenotypes.

\subsubsection{Drug screening and cardiac toxicity tests using hiPSCs}

Conventional drug studies use animal models and artificial manipulated cell systems to screen drugs for monogenic diseases, cancer, neurodegenerative diseases, congenital heart diseases as well as cardiovascular diseases (Rubin and Haston, 2011). Interestingly, 95\% of new drugs, which were screened using artificial manipulated cells, were withdrawn due to off-target effects and cardiac toxicity (Munos, 2009). During the last decades of extensive research in the field of cardiovascular medicine, few inventions have significantly improved the survival of heart failure patients (Chandrasekera and Pippin, 2015). The commonly prescribed treatments, such as $\beta$-adrenergic receptor blockers, act mainly by delayering the disease progression and could have severe side effects such as fainting, seizures or brachycardia (Gersh et al., 2011; Frishman, 2013). Current cardiac toxicity tests use mostly animal models or immortalized human cell lines overexpressing recombinant human Ether-à-go-go-Related Gene (hERG) channel to evaluate druginduced blockade of membrane channels leading to side effects of candidate drugs. Inhibition of hERG activity is the major cause for drug-induced arrhythmias and cardiac arrest because its inhibition causes LQTS which manifests by elongation of the QT interval. However, hERG assays have several limitations because the waveform of cardiac APs results from ions crossing the plasma membrane through a variety of other ion channels. Evaluation of APs from cell lines expressing recombinant hERG channels is not accurate enough for the recapitulation of the complex channel biology. Furthermore, recombinant expression systems are not able to mimic all side effects of drug candidates such as occurrence of organ toxicity. Until now hiPSC-derived CMs are the closed substitutes for human cardiac cells and thus appear to be a more predictive model for human cardiac toxicity. Comparative studies of hiPSC-derived CMs and hERG expressing HEK293 cells demonstrated that hiPSC-CMs were able to predict cardiac toxicity of multiple drugs including verapamil and alfuzosin in same efficiencies as their traditional 
counterparts (Liang et al., 2013). These data suggest that hiPSC-derived CMs are able to function as advanced model for the prediction of cardiac toxicity. Moreover, high-throughput platforms such as automatic robotic patch clamp and MEA systems can be used to screen large chemical libraries simultaneously in 96- or 384-formats for the discovery of novel drugs.

Recent studies of hiPSC-derived CMs from CPVT patients showed that the drug dantrolene, which was former administrated as muscle relaxant for the treatment of malignant hyperthermia, is able to prevent DADs in hiPSC-CMs from CPVT patients by restoring normal $\mathrm{Ca}^{2+}$ handling (Jung et al., 2012; Penttinen et al., 2015). Other studies with hiPSC-derived patient-CMs having mutations in lamin A/C (LMNA) showed that the cancer drugs $\mathrm{U} 0126$ and selumetinib block the MEK1 pathway, thereby improving the cell death of CMs with LMNA induced dilated cardiomyopathy (Siu et al., 2012).

\subsection{Cardiac differentiation of hiPSCs}

During the last 15 years, cardiac differentiation of pluripotent stem cells has been incredibly improved from originally 5-10\% to over 90\% efficiency (Burridge et al., 2012). Original protocols for the differentiation of $\mathrm{CMs}$ were based on spontaneous differentiation of three dimensional pluripotent stem cell aggregates called embryoid bodies (EBs) (Itskovitz-Eldor et al., 2000). To enrich the proportion of CMs using these protocols, percoll separation or cell sorting with cell surface markers such as VCAM1 was performed (Xu et al., 2002; van Hoof et al., 2010; Uosaki et al., 2011).

Over the past years, numerous direct differentiation methods were developed for CMs. To this end, hiPSCs are cultured in a 2D monolayer and growth factors or small molecules are directly added to the medium to induce directed differentiation into the cardiac lineage. In contrast to EBs, 2D monolayer techniques allow scalable differentiation for large quantities of $\mathrm{CMs}$ with yields around $85-95 \%$. Culturing differentiated CMs in medium containing lactate instead of glucose was found to favor the survival of CMs instead of other cell types. For this reason, purification of differentiated CMs is facilitated using these distinct metabolic differences of lactate and glucose metabolism between $\mathrm{CMs}$ and other differentiated cell types, thereby generating high yields of pure CMs (Tohyama et al., 2013).

During CM differentiation, canonical WNT/ $\beta$-catenin signaling acts in a biphasic role. Activation of WNT signaling induces mesodermal commitment and the following inhibition of WNT induces the formation of cardiac mesoderm (Paige et al., 2010). Treatment with special WNT inhibitors is able to boost cardiac development. However, suppression of WNT signaling at early stages or stimulation of WNT after mesoderm 
formation decreases the yield of CMs significantly. First studies focused on the manipulation of signaling pathways by mimicking embryonic developmental signals that control mesoderm induction such as application of activin, BMP, and FGF and subsequent inhibition of WNT, BMP and TGF $\beta$ pathways (Kattman et al., 2011; Burridge et al., 2012; Willems et al., 2012).

In 2012, it was demonstrated that differentiation of CMs could be started by activation of canonical WNT/ $\beta$-catenin signaling through addition of GSK3-inhibitor CHIR99021 to the culture medium RPMI together with B27 supplement without insulin. Suppression of WNT/ $\beta$-catenin signaling after mesoderm formation is sufficient for development of CMs. Inhibition of WNT signaling is achieved by using WNT inhibitor DKK1, or chemically produced small molecules, such as IWP2, IWP4 or IWR1 (Ren et al., 2011; Uosaki et al., 2011; Lian et al., 2012, 2012; Willems et al., 2012). Furthermore, it was shown that CM differentiation and culture is even successful using just RPMI medium containing ascorbic acid and human albumin in combination with the appropriate GSK3 inhibitor for two days following by the WNT inhibitor for the next two days (Burridge et al., 2014). In general, activation of canonical WNT signaling facilitate the transcription of essential mesodermal transcription factors such as brachyury which in turn stimulate MESP1 that functions as a master regulator of cardiogenic mesoderm formation. Inhibition of canonical WNT signaling through MESP1-activated gene DKK1 leads to upregulation of cardiac progenitor marker such as NKX2-5 and T-box transcription factor TBX5. In the final step of cardiac differentiation, NKX2-5 and TBX5 mediate the activation of connexin 40, NPPA and sarcomere-specific proteins which initiate the formation of atrial, ventricular and pacemaker/nodal CMs. Common cardiac differentiation protocols create a mixture of these cell types. 30-35 days after starting differentiation, most of the CMs develop into a ventricular-like phenotype followed by small amounts of atrial and pacemaker-like CMs (Burridge et al., 2014). Treatment with retinoic acid (RA) can increase the proportion of atrial-like $\mathrm{CMs}$ during $\mathrm{CM}$ differentiation, whereas inhibition of RA signaling increases the proportion of ventricular-like phenotypes (Zhang et al., 2011). Inhibition of NRG1ß/ERBB signaling can enhance the population of nodal-like CMs (Zhu et al., 2010) (Fig. 4).

In future, nodal-like CMs could be potentially utilized for the formation of biological pacemakers in patients, while ventricular CMs may be used for recovery from myocardial infarction. However, many issues, including tumorigenesis of remaining hiPSCs, further maturation of $\mathrm{CMs}$ and immune reaction of the graft, have to be addressed until hiPSCderived CMs could be useful for clinical applications (Lee et al., 2009; Pearl et al., 2011). Moreover, optimal grafts will require 3D-engineered tissues that are composed of different cell types such as cardiomyocytes, endothelial cells and fibroblasts. Sufficient vascularization will keep the graft alive and electrical coupling with existing CMs would 
minimize additional graft-induced arrhythmias (Caspi et al., 2007; Boudoulas and Hatzopoulos, 2009).



Figure 4. Key steps sufficient for cardiac differentiation of hiPSCs. Cardiogenesis is induced by canonical WNT, activin, BMP and FGF signaling and inhibited by exposure to insulin. First mesodermal markers include brachyury, eomesodermin, FOXC1 and MIXL1. Specification of cardiac progenitors is promoted by non-canonical WNTs and inhibited by canonical WNT, activin and BMP signaling. NKX2-5 and TBX5 co-regulate together with other factors to further cardiac differentiation. Neurogenin (NRG1), RA and specific microRNAs direct specification of cardiomyocyte subtypes. Maturation of differentiated $\mathrm{CMs}$ is not fully understood, but could be enhanced by hormones, electrical and mechanical stimulation or organized 3D structures of engineered heart tissues (Cyganek et al., 2013).

\subsection{Gene-editing technologies}

The development of nuclease-mediated gene editing technologies, including zinc finger nucleases (ZFNs), transcription activator-like nucleases (TALENs), and the clustered regularly interspaced short palindromic repeats (CRISPR)/CRISPR-associated protein 9 (Cas9) systems have introduced a new era for gene targeting, especially in the field of hiPSCs. Nuclease-mediated gene editing possesses various advantages compared to the conventional homologous recombination (HR)-mediated gene targeting which was historically limited to mouse ESCs. New nuclease-mediated genome editing tools are less time-consuming and facilitate higher efficiencies of gene targeting at endogenous loci. In principle, DNA double strand breaks (DSBs) are precisely introduced by nucleases which active the endogenous cellular DNA repair machinery to facilitate desired genomic modifications (Jasin, 1996; Vasquez et al., 2001). DSBs can be repaired by either error prone non-homologous end joining (NHEJ) or homology-directed repair (HDR) pathways. NHEJ repair often leads to small insertions or deletions (indels) at the targeted site, while the less frequent HDR pathway leads to perfect repair or precise genetic modification, but requires a homology-containing donor DNA sequence as repair template. HDR can be utilized to repair specific genetic loci; by contrast, NHEJ can be used to disrupt or abolish the function of target genes, for example, by frame-shifting indels. While these sitespecific nuclease technologies have made important advances in genetic engineering, each system has associated with advantages and disadvantages such as costs and 
difficulty of synthesis. In 2001, first ZFNs-mediated gene targeting was reported in living cells by injection of ZFN plasmids together with exogenous DNA fragments into the oocyte nuclei of Xenopus laevis (Bibikova et al., 2001; Bibikova et al., 2003). ZFNs consist of two domains: a DNA recognition domain and a nonspecific DNA cleavage domain of Fokl endonuclease (Kim et al., 1996). The DNA recognition domain contains three or more $\mathrm{Cys}_{2} \mathrm{His}_{2}$ zinc fingers, and each finger interacts with three consecutive DNA base pairs (bps) (Smith et al., 2000). The Fokl endonuclease is activated as a dimer when two individual ZFNs heterodimerize in an inverted orientation at the particular genomic locus to produce DSBs in the target DNA region.

TALENs were reported as a new technique for sequence specific gene targeting in 2010 (Christian et al., 2010). Similar to ZFNs, TALENs are composed of a TALE DNA binding domain and a Fokl endonuclease that induces DSBs. The central TALE DNA binding domain is composed of 33-35 aa repeats. These repeats only differ from each other by two aa, known as the repeat-variable di-residue. To engineer DNA-binding domains with novel DNA-binding specificities, individual TALEN repeats are assembled into an array that is designed to recognize the target DNA sequence. TALENs present several advantages over ZFNs. First, ZFNs only recognize three continuous base pairs, while each repeat in TALENs binds to a single bp. For this reason, TALENs can theoretically target any DNA sequence. Second, TALENs have comparable or higher efficiency to ZFNs. In addition, offtarget effects as well as cytotoxicity are very low (Hockemeyer et al., 2011; Moore et al., 2012). Until now, TALEN-mediated gene editing, including genetic deletions or insertions, has been successfully used in various mammals, such as rats (Tesson et al., 2011), mice (Sung et al., 2013), rabbit (Song et al., 2013), human cell lines (Hockemeyer et al., 2011) and monkeys (Liu et al., 2014b).

In 2013, the novel RNA-guided endonuclease system CRISPR/Cas9 from Streptococcus pyogenes was introduced as latest tool for genome engineering and acquired popularity as new revolution in biomedical research (Cong et al., 2013). The first CRISPR repeats were discovered when the iap gene from E. coli was sequenced (Ishino et al., 1987). Then, these CRISPR repeats were identified in most archaea and bacteria and functioned together with CRISPR-associated (Cas) genes as acquired immunity to eliminate invading genetic material from viruses and phages by specific DNA recognition and cleavage (Barrangou et al., 2007; Brouns et al., 2008). Based on the diversity of Cas proteins, CRISPR/Cas systems were classified into three categories: type I, II and III. The type II system requires just one Cas protein (Cas9) and is therefore chosen for genome editing techniques (Makarova et al., 2011). The Cas9 endonuclease forms together with two naturally occurring RNA species, CRISPR RNA (crRNA) and trans-activating CRISPR RNA (tracrRNA), a complex that recognizes complementary sequences. This CRISPR target 
sequence includes 20-24 bps and is located in close proximity to the protospacer adjacent motif (PAM) that resembles a 5'-NGG-3' sequence. After binding to the target region, Cas9 introduces specifically DSBs by the $\mathrm{HNH}$ nuclease domain and RuvC-like nuclease domain (Haurwitz et al., 2010; van der Oost et al., 2014) (Fig. 5).



Figure 5. Genome editing using CRISPR/Cas9. CRISPR/Cas9 is a RNA-guided DNA endonuclease system, in which Cas9 endonuclease forms a complex with the guide RNA consisting of two RNA species called crRNA and tracrRNA. This complex targets specific DNA sequences complementary to the 20-24 bp guide RNA sequence including a PAM motif and introduces specifically DSBs by Cas9. DSBs are repaired through either NHEJ or HDR. NHEJ repair often leads to indels at the targeted site, while HDR pathway leads to perfect repair or precise genetic modification (Modified from www.neb.com).

In comparison to previous genome editing tools, the design of the CRISPR/Cas9 system is easier as well as the targeting efficiency of the genome is reported to be higher (Ding et al., 2013). Especially, hiPSCs were difficult to be engineered using classical genome editing strategies (Hockemeyer and Jaenisch, 2010). First reports of CRISPR/Cas9 demonstrated targeted efficiencies between $2 \%$ and $4 \%$ of hiPSCs when assayed by deep sequencing (Mali et al., 2013). Like the TALEN technique, CRISPR/Cas9 was applied in various species for the generation of gene knockout (KO) and site-specific knockin, thereby other genomic editing methods such as ZFN and TALEN were often replaced due to the easier application of CRISPR/Cas9. Recent studies have observed off-target cleavage by CRISPR/Cas9 with up to 5 mismatches in regions that are similar to CRISPR/Cas9 binding sites (Fu et al., 2013). To improve the specificity of CRISPR/Cas9, a modified Cas9 (Cas9n) was developed which induces only single strand DNA breaks, also known as nicks. To introduce a DSB with this technique, it is necessary to have two Cas9n-induced nicks adjacent to each other, which greatly reduces off target-effects (Ran et al., 2013). Furthermore, CRISPR/Cas9 can be fused to protein complexes and provide a new platform for targeted gene inhibition (CRISPRi), activation (CRISPRa), as well as spatiotemporal or conditional gene regulation with higher specificity and reproducibility compared to 
systems such as RNA interference or tamoxifen-induced gene expression (Larson et al., 2013; Yang et al., 2013; Gersbach and Perez-Pinera, 2014; Nihongaki et al., 2015).

Although CRISPR/Cas9 is reported to be more efficient than ZFN and TALENs, general target efficiency of nuclease-induced genome editing is highly variable in different genes. For instance, one report described that ZFN technique was able to target OCT4 with over 90\% efficiency, while target efficiency of the gene PIX3 (paired-like homeodomain 3) reached only $8-11 \%$ (Hockemeyer et al., 2009).

\subsubsection{Application of gene editing in hiPSCs}

Genome-editing of hiPSCs by CRISPR/Cas9 provides a clinically relevant platform in order to understand human development and to investigate pathophysiology of diseases. CRISPR/Cas9 and other gene editing tools have been applied in various hESC- and hiPSCbased disease models and enable the creation of isogenic hESC or hiPSC lines that differ only at specific loci, while all other genomic locations remain unchanged. Consequently, the effects of the introduced mutation can be dissected from modifying effects of different genetic backgrounds. CRISPR/Cas9-mediated genome editing can be utilized to correct patient-specific monogenetic disease-causing mutations in hiPSC models. In contrast to previous methods using allele-specific downregulation of the disease-causing allele by RNA interference, CRISPR/Cas9-mediated correction of mutations is able to change the genome permanently, thereby generating isogenic controls with a persistent healthy phenotype in differentiated hiPSC-derived cell types (Chang et al., 2015; Huang et al., 2015; Li et al., 2016). These published studies provide mostly a proof of principle that the observed phenotypes are caused by a specific mutation. Karakikes et al. corrected the PLB gene mutation R14del, which was associated with impaired $\mathrm{Ca}^{2+}$ handling and PLB distribution. TALEN-based correction of the PLB mutation improved abnormalities significantly (Karakikes et al., 2015). Genome editing can be also used to introduce disease-causing mutations into hiPSCs from healthy donors to study the mechanisms of extremely rare disorders. HiPSC technology in combination with genome editing can be utilized for genetic screening to identify essential genes that interfere with specific cellular processes. For example, Hinson et al. were able to generate cardiac microtissues using hiPSC-derived CMs to evaluate the pathogenic mechanism of truncated titin gene variants, which are associated with dilated cardiomyopathy. Truncated titin variants were introduced into healthy hiPSCs, which were differentiated into cardiac microtissues. These microtissues had diminished contractile abilities and impaired responses to mechanical and adrenergic stress among other defects (Hinson et al., 2015). Missense mutations in the $H B B$ gene causing sickle cell disease and in the JAK3 gene causing severe 
combined immunodeficiency were successfully genetically corrected using CRISPR/Cas9 and healthy phenotypes were observed (Chang et al., 2015; Huang et al., 2015; Li et al., 2016). To overcome difficulties of gene corrections based on vulnerable hiPSCs, reprogramming and episomal vectors as well as CRISPR/Cas9 components were simultaneously introduced into dermal fibroblasts and generated gene-corrected hiPSCs in one step from patients suffering from fibrodysplasia ossificans progressiva syndrome (Kim et al., 2016). Despite these diverse application possibilities of engineered hiPSCs, genome editing utilized in complex polygenetic disorders will be a greater task because each patient may harbor a substantial number of functionally connected but unknown mutations that interfere with the disease phenotype.

\subsection{Aim of this thesis}

The aim of this project was to establish an in vitro cell culture system using the patientspecific hiPSC technology as a disease model to study the molecular mechanisms of CPVT. Six CPVT patients carrying heterozygous missense mutations (R420W, A2254V, E4076K, and $\mathrm{H} 4742 \mathrm{Y}$ ) in the 4 different domains of the $R Y R 2$ gene were recruited in the study. The scientific and technological objectives of the work included:

1. Generation and characterization of CPVT-hiPSC lines from 3 different patients having mutations in RYR2 at two different loci (A2254V, E4076K)

2. Direct differentiation of CPVT- and Ctrl-hiPSCs into functional CMs

3. Analysis of RYR2 expression and phosphorylation of hiPSC-derived CPVT-CMs compared to Ctrl-CMs

4. Genome editing by CRISPR/Cas9 using single-stranded oligodeoxynucleotides (sSODNs) to facilitate the introduction of mutations into CPVT-hiPSCs and/or the generation of isogenic controls having a potential healthy phenotype

5. Differentiation and characterization of CRISPR/Cas9-engineered hiPSC-derived CMs 


\section{Materials and Methods}

\subsection{Materials}

\subsubsection{Laboratory equipment}

Table 1: List of laboratory equipment

\begin{tabular}{|c|c|c|}
\hline Description & Name & Provider \\
\hline Autoclave & Sterilizator SN30 & Memmert \\
\hline \multirow[t]{2}{*}{ Balances } & Extend ED153-CW & Sartorius \\
\hline & CPA225D & Sartorius \\
\hline Blotting chamber & Mini Trans-Blot Cell & Bio-Rad \\
\hline \multirow[t]{3}{*}{ Centrifuges } & $5810 R$ & Eppendorf \\
\hline & 5415D & Eppendorf \\
\hline & $5415 R$ & Eppendorf \\
\hline Cell stimulator & MyoPacer ES & IonOptix \\
\hline \multirow{2}{*}{$\begin{array}{l}\text { Chemiluminescence } \\
\text { detection system }\end{array}$} & ChemiDoc MP Imaging & Bio-Rad \\
\hline & System Universal Hood III & \\
\hline \multirow[t]{2}{*}{ Confocal microscope } & LSM 710 confocal & Carl Zeiss \\
\hline & microscopic system & \\
\hline \multirow[t]{3}{*}{ Cooling units } & $4^{\circ} \mathrm{C}$ Comfort & Liebherr \\
\hline & $-20^{\circ} \mathrm{C}$ Premium & Liebherr \\
\hline & $-80^{\circ} \mathrm{C}$ VIP series & Sanyo \\
\hline Counting chamber & Thoma & Marienfeld Superior \\
\hline DNA Isolation & DNA Maxwell 16 System & Promega \\
\hline Electrophoresis & Mini-PROTEAN Tetra & Bio-Rad \\
\hline \multirow[t]{2}{*}{ chambers } & Vertical Electrophoresis & \\
\hline & Cell & \\
\hline Flow cytometer & FACS Canto II & $\mathrm{BD}$ \\
\hline Freezing box & Mr. Frosty & Thermo Fisher Scientific \\
\hline Gel documentation & Multilmage Light Cabinet & Alpha Innotech Corporation \\
\hline $\begin{array}{l}\text { Heated magnetic } \\
\text { stirrer }\end{array}$ & MR $3001 \mathrm{~K}$ & Heidolph \\
\hline Ice machine & Ziegra Ice maker & Ziegra Eismaschinen \\
\hline Incubators & BBD6620 & Heraeus Instruments \\
\hline \multirow[t]{4}{*}{ Microscopes } & Axio Oberserver A1 & Carl Zeiss \\
\hline & Axio Oberserver Z1 & \\
\hline & Primo Vert & \\
\hline & Axiovert 25 & \\
\hline
\end{tabular}




\begin{tabular}{lll}
\hline Microtome & - & Leica Biosystems \\
\hline NanoDrop & $\begin{array}{l}\text { 2000c/2000 UV-Vis } \\
\text { Spectrophotometer }\end{array}$ & Thermo Fisher Scientific \\
\hline PCR cycler & Thermocycler 48 & SensoQuest \\
\hline pH meter & inoLab pH 7110 & WTW \\
\hline Photometer & 96-well photometer & Bio-tek Instruments \\
\hline Pipet controller & Accu-jet pro & Brand \\
\hline Pipettes & Reference, Research plus & Eppendorf \\
& (10/100/1000 $\mu$ I) & \\
\hline Power supply & Power Pac 3000-Power & Bio-Rad \\
& supply & \\
\hline Shaker & Polymax 1040 & Heidolph \\
\hline Sterile work bench & MSC-Advantage series & Thermo Fisher Scientific \\
\hline Tissue embedding & Benchtop Tissue Processor & Leica Biosystems \\
system & 1020 & \\
\hline Transfection unit & Amaxa Nucleofector II & Lonza \\
& device & \\
\hline Vortexer & Vortex-Genie 2 & VWR \\
\hline Water bath & Type 003-2702 & Haake \\
\hline Water preparation & Milli-Q Reference & Merck Millipore \\
system & & \\
\hline
\end{tabular}

\subsubsection{Disposable Items}

Table 2: List of disposable items

\begin{tabular}{lll}
\hline Name & Type & Provider and Order number \\
\hline Cell culture plates & 6-cm dish, TC-treated & CytoOne Starlab \#CC7682-3359 \\
& 6-cm dish, untreated & Sarstedt \#82.1194.500 \\
& 10-cm dish, TC-treated & CytoOne Starlab \#CC7682-3394 \\
& 6-well plate, TC- treated & CytoOne Starlab \#CC7682-7506 \\
& 12-well plate, TC-treated & CytoOne Starlab \#CC7682-7512 \\
& 24-well plate, TC-treated & CytoOne Starlab \#CC7682-7524 \\
& 48-well plate, TC-treated & Eppendorf \#0030723112 \\
& 96-well plate, TC-treated & Eppendorf \#0030730119 \\
\hline Cell scraper & 2-Posit. Blade 25 & Sarstedt \#83.1830 \\
\hline Centrifuge tubes & $0.2 \mathrm{ml}, 0.5 \mathrm{ml}, 1.5 \mathrm{ml}, 2 \mathrm{ml}$ & Eppendorf \\
\hline Cryo tubes & 2 ml & Greiner \#126263 \\
\hline Falcon tubes & 15 ml & Sarstedt \#62.554.002 \\
& $50 \mathrm{ml}$ & Sarstedt \#62.547.004 \\
\hline
\end{tabular}




\begin{tabular}{|c|c|c|}
\hline Filcons & $50 \mu \mathrm{m}$ Filters & $\begin{array}{l}\text { Süd-Laborbedarf Gauting } \\
\# 150.47 \text { s }\end{array}$ \\
\hline Filter tips & $0.1-1000 \mu \mathrm{l}$ & $\begin{array}{l}\text { Starlab \#S1120-3810, } \\
\text { \#S1122-1830, \#S1120-1840 }\end{array}$ \\
\hline $\begin{array}{l}\text { Flow cytometry } \\
\text { tube }\end{array}$ & $\begin{array}{l}5 \mathrm{ml} \text { Polystyrene Round- } \\
\text { Bottom Tubes }\end{array}$ & BD Falcon \#352058 \\
\hline Pipette tips & $0.1-1000 \mu \mathrm{l}$ & $\begin{array}{l}\text { Starlab \#S1111-3700, } \\
\text { \#S1111-1706, \#S1112-1720 }\end{array}$ \\
\hline Pipettes & $5 \mathrm{ml}, 10 \mathrm{ml}, 25 \mathrm{ml}$ & $\begin{array}{l}\text { Sarstedt \#86.1253.001, } \\
\# 86.1254 .001, \# 86.1685 .001\end{array}$ \\
\hline PVDF membrane & $\begin{array}{l}\text { Amersham Hybond } \mathrm{P} \\
\text { Western blotting membranes }\end{array}$ & Sigma-Aldrich \#GE10600023 \\
\hline $\begin{array}{l}\text { Slides and } \\
\text { coverslips }\end{array}$ & $\begin{array}{l}76 \times 26 \mathrm{~mm} \\
18 \times 18 \mathrm{~mm} \\
\text { Round } 25 \mathrm{~mm}\end{array}$ & $\begin{array}{l}\text { Thermo Fisher Scientific } \\
\# 10143562 C E, \\
\text { Thermo Fisher Scientific } \\
\# 4004672 \\
\text { R. Langenbrinck, \#1049251 }\end{array}$ \\
\hline Sterile filters & $\begin{array}{l}\text { Millex-GS, } 0.22 \mu \mathrm{m} \\
\text { Steriflip } 50 \mathrm{ml} 0.22 \mu \mathrm{m} \\
\text { Steritops } 500 \mathrm{ml} 0.22 \mu \mathrm{m}\end{array}$ & $\begin{array}{l}\text { Merck Millipore \#GLGS0250S } \\
\text { Merck Millipore \#SCGP00525 } \\
\text { Merck Millipore \# SCGPT05RE }\end{array}$ \\
\hline $\begin{array}{l}\text { Whatman gel } \\
\text { blotting paper }\end{array}$ & Grade GB003 & Sigma-Aldrich \#WHA10426890 \\
\hline
\end{tabular}

\subsubsection{Plasmids and oligonucleotides}

Combined CRISPR/Cas9-Plasmids (Fig. 6) were designed and generated from SigmaAldrich and listed in Table 3.

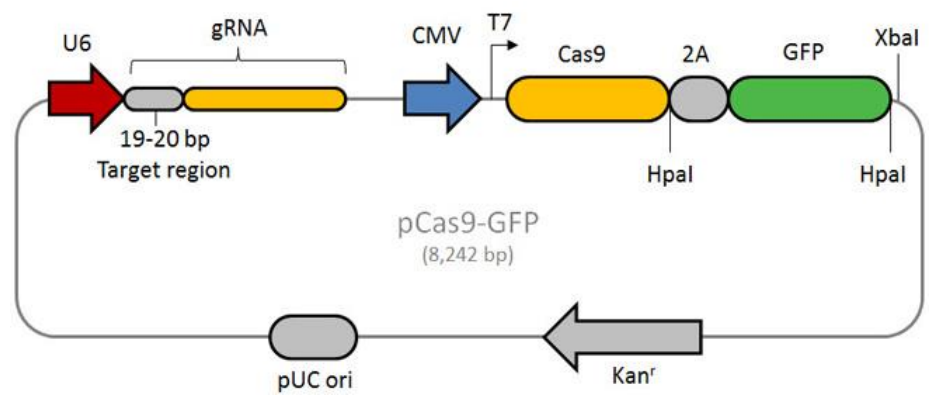

Figure 6. CRISPR/Cas9 plasmid containing the sequence of gRNA, Cas9, GFP and kanamycin.

All oligonucleotides were synthesized from Microsynth AG. The sequences are listed in alphabetical order together with the amplified fragment length (F), annealing temperature (TA), and number of cycles. Table 4 shows the list of oligonucleotides used for reverse transcription PCR. Table 5 shows oligonucleotides used for genome editing 
with CRISPR/Cas9. Table 6 shows oligonucleotides used for the screening of CRISPR/Cas9 clones. Table 7 shows oligonucleotides used for allele specific sequencing.

Table 3: List of CRISPR/Cas9-plasmids

\begin{tabular}{|c|c|c|c|}
\hline Name & CRISPR-binding site & $\begin{array}{l}\text { Calculated } \\
\text { Off-targets }\end{array}$ & Order Number \\
\hline CRISPR-B-1/Cas9 & $\begin{array}{l}\text { CCGGATCTAGAAAAGGTG } \\
\text { AGCA }\end{array}$ & 1 at $n=3$ & humanRyr2B_0_18_CCG \\
\hline CRISPR-C-1/Cas9 & $\begin{array}{l}\text { ACGAACTCTTCGTAGTCG } \\
\text { AGGG }\end{array}$ & 0 at $n=3$ & $\begin{array}{l}\text { HS0000065369, } \\
\text { predesigned }\end{array}$ \\
\hline
\end{tabular}

Table 4: List of oligonucleotides for reverse transcription-PCR analysis

\begin{tabular}{|c|c|c|c|c|}
\hline Name & Sequence & $\begin{array}{l}F \\
{[b p]}\end{array}$ & $\mathrm{T}_{\mathrm{A}}\left[{ }^{\circ} \mathrm{C}\right]$ & Cycles \\
\hline \multirow[t]{2}{*}{$A F P$} & For: ACTCCAGTAAACCCTGGTGTTG & 255 & 60 & 33 \\
\hline & Rev: GAAATCTGCAATGACAGCCTCA & & & \\
\hline \multirow[t]{2}{*}{$A L B$} & For: CCTTTGGCACAATGAAGTGGGTAACC & 355 & 62 & 35 \\
\hline & Rev: CAGCAGTCAGCCATTTCACCATAG & & & \\
\hline \multirow[t]{2}{*}{ CACNA1C } & For: ACCTGGAATGTCTGAAGCGA & 248 & 60 & 30 \\
\hline & Rev: TTTCTCACTGGACTCGACCC & & & \\
\hline \multirow[t]{2}{*}{ CASQ2 } & For: GGTCACGCAAAAACAGTTCC & 284 & 60 & 30 \\
\hline & Rev: CGAAGGCTTGGACTTCCAGT & & & \\
\hline \multirow[t]{2}{*}{ cTNT } & For: GACAGAGCGGAAAAGTGGGA & 305 & 56 & 35 \\
\hline & Rev: TGAAGGAGGCCAGGCTCTAT & & & \\
\hline \multirow{2}{*}{ FOXD3 } & For: GTGAAGCCGCCTTACTCGTAC & 353 & 60 & 38 \\
\hline & Rev: CCGAAGCTCTGCATCATGAG & & & \\
\hline \multirow[t]{2}{*}{ GAPDH } & For: AGAGGCAGGGATGATGTTCT & 258 & 60 & 30 \\
\hline & Rev: TCTGCTGATGCCCCCATGTT & & & \\
\hline \multirow[t]{2}{*}{ GDF3 } & For: TTCGCTTTCTCCCAGACCAAGGTTTC & 331 & 54 & 30 \\
\hline & Rev: TACATCCAGCAGGTTGAAGTGAACAGCACC & & & \\
\hline \multirow[t]{2}{*}{ IP3R1 } & For: GACCTTCGGGACGAAGAGAG & 230 & 60 & 30 \\
\hline & Rev: AATGCTTTCATGGAACACTCGGTC & & & \\
\hline \multirow[t]{2}{*}{ IP3R2 } & For: AGCAACATCCAAAGCATATTGTGT & 198 & 59 & 30 \\
\hline & Rev: AATGCTTTCATGGAACACTCGGTC & & & \\
\hline \multirow[t]{2}{*}{ LIN28 } & For: AGTAAGCTGCACATGGAAGG & 410 & 52 & 30 \\
\hline & Rev: ATTGTGGCTCAATTCTGTGC & & & \\
\hline \multirow[t]{2}{*}{$M L C 2 A$} & For: GAAGGTGAGTGTCCCAGAGG & 289 & 58 & 30 \\
\hline & Rev: CTTGTAGTCGATGTTCCCCG & & & \\
\hline$M L C 2 V$ & For: GGCGAGTGAACGTGAAAAAT & 200 & 56 & 30 \\
\hline
\end{tabular}




\begin{tabular}{|c|c|c|c|c|}
\hline & Rev: CAGCATTTCCCGAACGTAAT & & & \\
\hline \multirow[t]{2}{*}{ NANOG } & For: AGTCCCAAAGGCAAACAACCCACTTC & 164 & 64 & 36 \\
\hline & Rev: ATCTGCTGGAGGCTGAGGTATTTCTGTCTC & & & \\
\hline \multirow[t]{2}{*}{ OCT4 } & For: GACAACAATGAAAATCTTCAGGAGA & 113 & 54 & 34 \\
\hline & Rev: TTCTGGCGCCGGTTACAGAACCA & & & \\
\hline \multirow[t]{2}{*}{ RYR2 } & For: ATCCACAAAGAACAACAGAAGCTAT & 509 & 60 & 30 \\
\hline & Rev: CCATAAGACAAGTGCAAGTACCTTT & & & \\
\hline \multirow[t]{2}{*}{ SOX2 } & For: ATGCACCGCTACGACGTGA & 437 & 60 & 34 \\
\hline & Rev: CTTTTGCACCССТCССАTTT & & & \\
\hline \multirow[t]{2}{*}{ SYN } & For: TGCAGAACAAGTACCGAGAG & 297 & 57 & 34 \\
\hline & Rev: CTGTCTCCTTAAACACGAACC & & & \\
\hline \multirow[t]{2}{*}{$\mathrm{TH}$} & For: GCGGTTCATTGGGCGCAGG & 215 & 60 & 34 \\
\hline & Rev: CAAACACCTTCACAGCTCG & & & \\
\hline \multirow[t]{2}{*}{$\alpha-A C T$} & For: AGGAGGAAGAATGGCCTGAT & 291 & 60 & 30 \\
\hline & Rev: GATGCAGTACTGGGCCTGAT & & & \\
\hline \multirow[t]{2}{*}{$\alpha-M H C$} & For: GTCATTGCTGAAACCGAGAATG & 413 & 60 & 35 \\
\hline & Rev: GCAAAGTACTGGATGACACGCT & & & \\
\hline \multicolumn{5}{|c|}{ 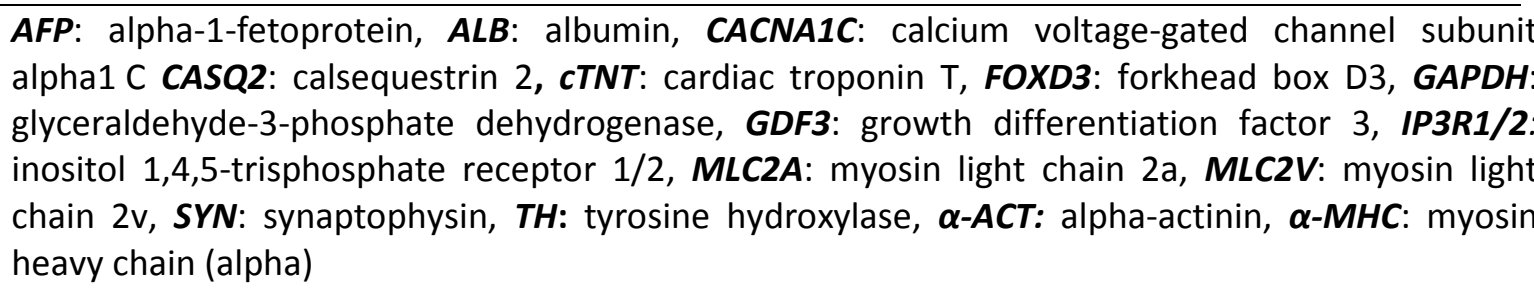 } \\
\hline
\end{tabular}

Table 5: Single-stranded oligodesoxynucleotides (ssODNs) used for CRISPR/Cas9 technology

\begin{tabular}{lll}
\hline Name & Sequence & Additional mutations \\
\hline CRISPR- & GTCAGAAACGGAATTCCTTTTGTC & Correction: G \\
Oligo-C & TTGTGCGGAGACGGATGAGAATG & Silent mutation for EcoRI: T>C \\
& AAACCCTCGACTACGAAGAGTTC & (TTT > TTC) \\
& GTCAAACGCTTCCACGAACCTGCG & \\
& AAGGACATCGGCTTCA & \\
\hline CRISPR- & GTCAGAAACGGAATTCTTTTGC & Correction: G \\
Oligo-c- & TTGTGCGGAGACGGATGAGAATG & Silent mutation for EcoRI: G >A \\
new1 & AAACTCTCGACTACGAAGAATTC & (GAG> GAA) \\
& GTCAAACGCTTCCACGAACCTGCG & Binding prevention at PAM: C >T \\
& AAGGACATCGGCTTCA & (ACC > ACT) \\
\hline CRISPR- & GTCAGAAACGGAATTTCTTTGTC & Correction: G \\
Oligo-C- & TTGTGCGGAGACGGATGAGAATG & Silent mutation for EcoRI: G>A \\
new2 & AAACTCTAGATTATGAAGAATTC & (GAG > GAA) \\
& GTCAAACGCTTCCACGAACCTGCG & Binding prevention at PAM: C>T \\
& AAGGACATCGGCTTCA & (ACC > ACT) \\
& & Additional binding prevention: \\
& & (CTC > CTA) \\
\hline
\end{tabular}




\begin{tabular}{|c|c|c|c|c|c|}
\hline \multirow{6}{*}{$\begin{array}{l} \\
\text { CRISPR- } \\
\text { Oligo-b-1 }\end{array}$} & & \multicolumn{4}{|c|}{$\begin{array}{l}(G A C>G A T) \\
(T A C>T A T)\end{array}$} \\
\hline & GCCTCCCCAGCTATGAGAGGTTC & \multicolumn{4}{|c|}{ Correction: $\mathrm{C}$} \\
\hline & AACACCACTGGATGTGGCTGCAG & \multicolumn{4}{|c|}{ CPVT-Mutation (A2254V) } \\
\hline & CTTCGGTGATGGATAATAATGAA & \multicolumn{4}{|c|}{ (GTA instead of GCA) leads to the } \\
\hline & CTAGCATTAGCTCTGCGTGAGCCG & \multicolumn{4}{|c|}{ generation of Spel ( $\underline{\text { ACTAGT}})$ restriction } \\
\hline & GATCTAGAAAAGGT & \multicolumn{4}{|l|}{ site } \\
\hline \multirow{2}{*}{\multicolumn{6}{|c|}{$\begin{array}{l}\text { The CRISPR/Cas9 binding site is highlighted in blue. Point mutations that should be correc } \\
\text { highlighted in in red. Additional mutations are marked in purple. Underlined sequen } \\
\text { recognized by restriction enzymes. } \\
\text { Table 6: List of oligonucleotides used for screening of CRISPR/Cas9-edited clones }\end{array}$}} \\
\hline & & & & & \\
\hline Name & \multicolumn{2}{|l|}{ Sequence } & $F[b p]$ & $\mathrm{T}_{\mathrm{A}}\left[{ }^{\circ} \mathrm{C}\right]$ & Cycles \\
\hline \multirow{2}{*}{$\begin{array}{l}\text { CPVTb-Seq } \\
(\mathrm{C} 90 / 91)\end{array}$} & \multicolumn{2}{|c|}{ For: ACACTATGGATGGTGTTTAGAA } & 560 & 57 & 35 \\
\hline & \multicolumn{2}{|l|}{ Rev: AGAACGTTGGTTCTCCTTCC } & & & \\
\hline CPVTc-Seq & \multicolumn{2}{|l|}{ For: TCTTCCAACAACGTGGAGAT } & 368 & 57 & 35 \\
\hline (C92/C93) & \multicolumn{2}{|l|}{ Rev: TAATTCAGGACGCTCTCTGC } & & & \\
\hline \multirow[t]{2}{*}{ EcoRI-g } & \multicolumn{2}{|c|}{ For: CCAGGCTTTGTGGAGGTAGG } & 865 & 60 & 35 \\
\hline & \multicolumn{2}{|c|}{ Rev: GGGTGAAGTCCTGTCTGAGC } & & & \\
\hline \multirow[t]{2}{*}{ RYR2c-1021 } & \multicolumn{2}{|c|}{ For: AGAGTGAGAGATAAACAGGGACAT } & 1021 & 59 & 40 \\
\hline & \multicolumn{2}{|c|}{ Rev: GGATGAAAAGAAGGCCGTGAC } & & & \\
\hline
\end{tabular}

Table 7: List of oligonucleotides for allele-specific sequencing

\begin{tabular}{lllll}
\hline Name & Sequence & $\mathbf{F}(\mathbf{b p})$ & $\left.\mathbf{T}_{\mathbf{A}}{ }^{\circ} \mathbf{C}\right]$ & Cycles \\
\hline RYR2-A-new & $\begin{array}{l}\text { For: GTGGACGTGAAATCCGTGAGA } \\
\text { Rev: GGGGTGGAAGTAGCCAATGAG }\end{array}$ & 267 & 60 & 25 \\
& For: AGTGTTGGTCTTGCCTCCC & 267 & 60 & 25 \\
& Rev: CTCCACACTCTCCCCATTACAG & & & \\
\hline RYR2-B-RT & For: CGATGGCAAGGGAGTCATTTC & 267 & 57 & 28 \\
& Rev: AGGACGCTCTCTGCTAATTCC & & & \\
\hline RYR2-D-RT & $\begin{array}{l}\text { For: AGCCAAAGAAAGACAGCTCCT } \\
\text { Rev: AATGCCACCACAGTGTATAGGT }\end{array}$ & 298 & 57 & 28 \\
& & & & \\
\hline
\end{tabular}

\subsubsection{Antibodies}

Primary and secondary antibodies are listed in Tables $\mathbf{8}$ and 9, respectively, and were used for immunofluorescence (IF) and western blot (WB) analyses. 
Table 8: List of primary antibodies

\begin{tabular}{|c|c|c|c|c|}
\hline Antigen & Host & Dilution & Blocking & Provider \\
\hline AFP & $\begin{array}{l}\text { Rabbit } \\
\text { (lgG) }\end{array}$ & IF: $1: 100$ & IF: $1 \%$ BSA/DPBS & Dako \#A0008-4oC \\
\hline cTNT & $\begin{array}{l}\text { Mouse } \\
(\operatorname{lgG} 1)\end{array}$ & $\begin{array}{l}\text { IF: } 1: 500 \\
\text { WB: } 1: 10.000 \text { in } 1 \% \\
\text { BSA/TBS-T }\end{array}$ & $\begin{array}{l}\text { IF: } 1 \% \text { BSA/DPBS } \\
\text { WB: } 3 \% \text { BSA/TBS-T }\end{array}$ & $\begin{array}{l}\text { Thermo Fisher } \\
\text { Scientific } \\
\text { \#MS295PABX 13-11 }\end{array}$ \\
\hline CX43 & $\begin{array}{l}\text { Rabbit } \\
(\lg G)\end{array}$ & IF: 1:1000 & IF: $1 \%$ BSA/DPBS & $\begin{array}{l}\text { Abcam } \\
\text { \#ab11370 (GJA1) }\end{array}$ \\
\hline GAPDH & $\begin{array}{l}\text { Rabbit } \\
\text { (IgG) }\end{array}$ & $\begin{array}{l}\text { WB: } 1: 5000 \text { in } 1 \% \\
\text { BSA/TBS-T }\end{array}$ & WB: $3 \%$ BSA/TBS-T & $\begin{array}{l}\text { Thermo Fisher } \\
\text { Scientific \#PA1-987 }\end{array}$ \\
\hline IP3R & $\begin{array}{l}\text { Rabbit } \\
(\operatorname{lgG})\end{array}$ & $\begin{array}{l}\text { WB: } 1: 750 \text { in } 1 \% \\
\text { BSA/TBS-T }\end{array}$ & WB: $3 \%$ BSA/TBS-T & $\begin{array}{l}\text { Merck Millipore } \\
\# 07-1210\end{array}$ \\
\hline LIN28 & $\begin{array}{l}\text { Goat } \\
\text { (lgG) }\end{array}$ & IF: $1: 300$ & IF: $1 \%$ BSA/DPBS & $\begin{array}{l}\text { R\&D systems } \\
\text { \#AF3757 }\end{array}$ \\
\hline MLC2A & $\begin{array}{l}\text { Mouse } \\
\text { (lgG2b) }\end{array}$ & $1: 200$ & IF: $1 \%$ BSA/DPBS & $\begin{array}{l}\text { Synaptic Systems } \\
\# 311-011 \text { (56F5) }\end{array}$ \\
\hline MLC2V & $\begin{array}{l}\text { Rabbit } \\
(\lg G)\end{array}$ & IF: 1:200 & IF: $1 \%$ BSA/DPBS & $\begin{array}{l}\text { Protein tech } \\
\# 10906-1-A P\end{array}$ \\
\hline NANOG & $\begin{array}{l}\text { Goat } \\
(\lg G)\end{array}$ & IF: $1: 200$ & IF: $1 \%$ BSA/DPBS & Abcam \#PA5-18406 \\
\hline NCX1 & $\begin{array}{l}\text { Mouse } \\
\text { (lgG2b) }\end{array}$ & $\begin{array}{l}\text { WB: } 1: 1000 \text { in } 1 \% \\
\text { BSA/TBST }\end{array}$ & WB: $3 \%$ BSA/TBS-T & Novus \#NB300-127 \\
\hline OCT4 & $\begin{array}{l}\text { Goat } \\
(\lg G)\end{array}$ & IF. 1:40 & IF: $1 \%$ BSA/DPBS & $\begin{array}{l}\text { R\&D systems } \\
\text { \#AF1759 }\end{array}$ \\
\hline RYR2 & $\begin{array}{l}\text { Rabbit } \\
\text { (lgG) }\end{array}$ & $\begin{array}{l}\text { IF: } 1: 500 \\
\text { WB: } 1: 5000 \text { in } 1 \% \\
\text { BSA/TBS-T }\end{array}$ & WB: $3 \%$ BSA/TBS-T & $\begin{array}{l}\text { Sigma-Aldrich } \\
\text { Prestige \#HPA020028 }\end{array}$ \\
\hline RYR2 & $\begin{array}{l}\text { Mouse } \\
(\lg G)\end{array}$ & $\begin{array}{l}\text { WB: } 1: 1000 \text { in } 1 \% \\
\text { BSA/TBS-T }\end{array}$ & WB: $3 \%$ BSA/TBS-T & $\begin{array}{l}\text { Pierce antibodies } \\
\text { \#MA3-916 C3-33 }\end{array}$ \\
\hline $\begin{array}{l}\text { RYR2- } \\
\text { pSer2030 }\end{array}$ & $\begin{array}{l}\text { Rabbit } \\
\text { (IgG) }\end{array}$ & $\begin{array}{l}\text { WB: just unspecific } \\
\text { binding (1:300- } \\
1: 1000)\end{array}$ & 3-6\% BSA/TBS-T & Badrilla \#A010-32 \\
\hline $\begin{array}{l}\text { RYR2- } \\
\text { pSer2808 }\end{array}$ & $\begin{array}{l}\text { Rabbit } \\
(\lg G)\end{array}$ & $\begin{array}{l}\text { WB: } 1: 5000 \text { in } 3 \% \\
\text { BSA/TBS-T }\end{array}$ & WB: $6 \%$ BSA/TBS-T & Badrilla \#A010-30 \\
\hline $\begin{array}{l}\text { RYR2- } \\
\text { pSer2814 }\end{array}$ & $\begin{array}{l}\text { Rabbit } \\
(\lg G)\end{array}$ & $\begin{array}{l}\text { WB: } 1: 5000 \text { in } 3 \% \\
\text { BSA/TBS-T }\end{array}$ & WB: $6 \%$ BSA/TBS-T & Badrilla \#A010-31 \\
\hline $\begin{array}{l}\text { SERCA2 } \\
\text { ATPase }\end{array}$ & $\begin{array}{l}\text { Mouse } \\
\text { (IgG2a) }\end{array}$ & $\begin{array}{l}\text { WB: } 1: 1000 \text { in } \\
1 \% \text { BSA/TBST }\end{array}$ & WB: $3 \%$ BSA/TBS-T & $\begin{array}{l}\text { Thermo Fisher } \\
\text { Scientific \#MA3-919 }\end{array}$ \\
\hline SOX2 & Mouse & IF: $1: 50$ & IF: $1 \%$ BSA/DPBS & R\&D systems \\
\hline
\end{tabular}




\begin{tabular}{|c|c|c|c|c|}
\hline & $(\lg G)$ & & & \#MAB2018 \\
\hline SSEA-4 & $\begin{array}{l}\text { Mouse } \\
\text { (IgG) }\end{array}$ & IF: $1: 200$ & IF: $1 \%$ BSA/DPBS & Abcam \#MC813 \\
\hline TRA-1-60 & $\begin{array}{l}\text { Mouse } \\
(\operatorname{lgM})\end{array}$ & IF: $1: 200$ & IF: $1 \%$ BSA/DPBS & $\begin{array}{l}\text { R\&D systems } \\
\text { \#MAB4770 }\end{array}$ \\
\hline$\alpha$-ACTININ & $\begin{array}{l}\text { Mouse } \\
\text { (IgG1) }\end{array}$ & $\begin{array}{l}\text { IF: } 1: 1000 \\
\text { WB: } 1: 5000 \text { in } 2.5 \% \\
\text { nonfat dry milk in } \\
\text { TBS-T }\end{array}$ & $\begin{array}{l}\text { IF: } 1 \% \text { BSA/DPBS } \\
\text { WB: } 5 \% \text { nonfat dry } \\
\text { milk in TBS-T }\end{array}$ & Sigma-Aldrich \#A7811 \\
\hline$\alpha-S M A$ & $\begin{array}{l}\text { Mouse } \\
\text { (IgG2A) }\end{array}$ & IF: $1: 3000$ & IF: $1 \%$ BSA/DPBS & Sigma-Aldrich \#A2547 \\
\hline $\begin{array}{l}\beta-I I I- \\
\text { TUBULIN }\end{array}$ & $\begin{array}{l}\text { Mouse } \\
\text { (IgG2A/ } \\
\text { K) }\end{array}$ & IF: $1: 2000$ & IF: $1 \%$ BSA/DPBS & Covance \#MMS-435P \\
\hline
\end{tabular}

$\alpha$-SMA: $\alpha$ - smooth muscle actin, SSEA4: stage-specific embryonic antigen 4

Table 9: List of secondary antibodies

\begin{tabular}{|c|c|c|c|}
\hline $\begin{array}{l}\text { Fluorophore \& } \\
\text { Antigen }\end{array}$ & Host & Dilution & Company \\
\hline $\begin{array}{l}\text { Alexa Fluor 488- } \\
\text { anti-mouse }\end{array}$ & Donkey (IgG) & $1: 1000$ & Thermo Fisher Scientific \#A21202 \\
\hline $\begin{array}{l}\text { Alexa Fluor 555- } \\
\text { anti-goat }\end{array}$ & Donkey (IgG) & 1:1000 & Thermo Fisher Scientific \#A21432 \\
\hline $\begin{array}{l}\text { Alexa Fluor 647- } \\
\text { anti-mouse }\end{array}$ & Donkey (IgG) & $1: 1000$ & Thermo Fisher Scientific \#A31571 \\
\hline Cy3-anti-goat & $\begin{array}{l}\text { Donkey } \\
\text { (IgG) }\end{array}$ & $1: 500$ & Jackson ImmunoResearch \#705-165-147 \\
\hline Cy3-anti-mouse & $\begin{array}{l}\text { Goat } \\
(\lg G+\lg M)\end{array}$ & $1: 300$ & Jackson ImmunoResearch \#115-165-068 \\
\hline Cy3-anti-rabbit & $\begin{array}{l}\text { Goat } \\
\text { (IgG) }\end{array}$ & $1: 600$ & Jackson ImmunoResearch \#111-165-003 \\
\hline Cy5-anti-mouse & Donkey(lgG) & $1: 300$ & Jackson ImmunoResearch \#715-175-150 \\
\hline FITC anti-rabbit & Donkey (IgG) & $1: 200$ & Jackson ImmunoResearch \#711-095-152 \\
\hline FITC-anti-mouse & Goat (IgG) & $1: 100$ & Jackson ImmunoResearch \#115-096-072 \\
\hline HRP-anti-mouse & Donkey (IgG) & $1: 10.000$ & Thermo Fisher Scientific \#A16011 \\
\hline HRP-anti-rabbit & Donkey (IgG) & $1: 10.000$ & Thermo Fisher Scientific \#A16023 \\
\hline PE-anti mouse & Donkey (IgG) & $1: 200$ & Jackson ImmunoResearch \#703-116-155 \\
\hline
\end{tabular}




\subsubsection{Chemicals and reagents}

All chemicals, reagents, kits and enzymes used for molecular and protein biological experiments are listed in Table 10 together with the name of the provider.

Table 10: List of molecular biological reagents

\begin{tabular}{|c|c|}
\hline Name & Provider \\
\hline Agar & Serva Electrophoresis \#200201 \\
\hline Alkaline phosphatase staining kit & Sigma-Aldrich \#86R-1KT \\
\hline Ammonium persulfate (APS) & Roth \#9178 \\
\hline AmpliTaq DNA polymerase with buffer II & Thermo Fisher Scientific \#N808-0167 \\
\hline Boric acid & Sigma-Aldrich \#15663 \\
\hline Bromphenol blue & Roth \#A512.2 \\
\hline Calcium chloride dihydrate & Roth \#HN04 \\
\hline Calyculin A & Santa Cruz \#sc-24000 \\
\hline cOmplete (protease inhibitor cocktail tablets) & Roche \#04693132001 \\
\hline $\begin{array}{l}\text { DAPI (4', 6-Diamidino-2-phenylindole } \\
\text { dihydrochloride) }\end{array}$ & Sigma-Aldrich \#D9542 \\
\hline $\begin{array}{l}\text { Di-Sodium hydrogen phosphate dihydrate } \\
\left(\mathrm{Na}_{2} \mathrm{HPO}_{4} \cdot 2 \mathrm{H}_{2} \mathrm{O}\right)\end{array}$ & Roth \#T877 \\
\hline Dithiothreitol (DTT) & Roth \#6908 \\
\hline dNTP mix & Bioline \#BIO-39029 \\
\hline EcoRI-HF & New England Biolabs \#R3101S \\
\hline Ficoll 400 & Roth \#CN90.1 \\
\hline Fluoromount-G & eBioscience \#00-4958-02 \\
\hline Fluo 4/AM, cell permeant & Thermo Fisher Scientific \#F14201 \\
\hline Formalin (37 \%) & Merck Millipore \#1039991000 \\
\hline GeneRuler 100 bp Plus DNA Ladder & Thermo Fisher Scientific \#0321 \\
\hline Glucose & Sigma-Aldrich \#G8270 \\
\hline Glycerol & Roth \#3783 \\
\hline Glycine & Roth \#3908 \\
\hline GoTaq G2 DNA polymerase & Promega \#M7845 \\
\hline HEPES & Roth \#9105 \\
\hline Hydrochloric acid fuming $37 \%$ & Merck Millipore \#100317 \\
\hline IGEPAL CA-630 & Sigma-Aldrich \#I3021 \\
\hline Isoprenaline hydrochloride & Sigma-Aldrich \#I5627 \\
\hline Isopropanol & Merck Millipore \#109634 \\
\hline Kanamycin sulfate & Roth \#T832 \\
\hline
\end{tabular}




\begin{tabular}{|c|c|}
\hline $\mathrm{KCl}$ & Sigma-Aldrich \#P9541 \\
\hline$L-755,507$ & Tocris Bioscience \#2197 \\
\hline Luminol & Sigma-Aldrich \#123072 \\
\hline Magnesium chloride & Sigma-Aldrich \#M8266 \\
\hline Maxwell 16 cell DNA purification kit & Promega \#AS1020 \\
\hline Methanol & Merck Millipore \#106009 \\
\hline Midori Green Advance & Biozym \#617004 \\
\hline MuLV reverse transcriptase $(50 \mathrm{U} / \mu \mathrm{l})$ & Thermo Fisher Scientific \#N808-0018 \\
\hline Nonfat dry milk & Roth \#T145 \\
\hline Nuclease-free water & Thermo Fisher Scientific \#AM9932 \\
\hline Oligo $d(T) 16(50 \mu \mathrm{M})$ & Thermo Fisher Scientific \#N808-0128 \\
\hline Paraformaldehyde (PFA) & Sigma-Aldrich \#158127 \\
\hline P-coumaric acid & Sigma-Aldrich \#C9008 \\
\hline PeqGold protein marker $\mathrm{V}$ & Peqlab \#27-2210 \\
\hline peqGold universal agarose & Peqlab \#35-1020 \\
\hline $\begin{array}{l}\text { PhosStop (phosphatase inhibitor cocktail } \\
\text { tablets) }\end{array}$ & Roche $\# 04906837001$ \\
\hline Pierce BCA protein assay kit & Thermo Fisher Scientific \#23225 \\
\hline Plasmid Maxi Kit & Qiagen \#12162 \\
\hline Pluronic F-127 & Thermo Fisher Scientific \#P3000MP \\
\hline QIAquick gel extraction kit & Qiagen \#28706 \\
\hline QIAquick PCR purification kit & Qiagen \#28104 \\
\hline QuickExtract DNA Extraction Solution 1.0 & Biozym \#101094 \\
\hline RNase inhibitor $(20 \mathrm{U} / \mu \mathrm{l})$ & Thermo Fisher Scientific \#N808-0119 \\
\hline Rotiphorese gel 30 & Roth \#3029 \\
\hline Sodium azide & Sigma-Aldrich \#S2002 \\
\hline Sodium chloride & Roth \#9265.1 \\
\hline Sodium dodecyl sulfate (SDS) & Roth \#2326 \\
\hline Sodium fluoride (NaF) & Roth \#P756 \\
\hline Spel-HF & New England Biolabs \#R3133S \\
\hline SV total RNA isolation system & Promega \#Z3105 \\
\hline Tetramethylethylenediamine (TEMED) & Roth \#2367 \\
\hline Tris & Roth \#5429 \\
\hline Triton X-100 & Sigma-Aldrich \#3051.3 \\
\hline Trypton & Roth \#6681.1 \\
\hline Tween 20 & Bio-Rad \#170-6531 \\
\hline Yeast extract & Roth \#2363.1 \\
\hline
\end{tabular}




\subsubsection{Solutions, buffers and bacteria material for molecular and protein analyses}

Table 11: List of components for molecular biological methods, and protein analyses

\begin{tabular}{|c|c|}
\hline Solution & Components \\
\hline \multirow[t]{4}{*}{ 1x Transfer buffer } & $32 \mathrm{mM}$ Tris, $\mathrm{pH} 8.3$ \\
\hline & 19 mM Glycine \\
\hline & $10 \%$ Methanol \\
\hline & $0.05 \%$ SDS \\
\hline \multirow[t]{5}{*}{ 2x HBS solution } & $280.0 \mathrm{mM} \mathrm{NaCl}$ \\
\hline & 50.0 mM HEPES \\
\hline & $0.15 \mathrm{mM} \mathrm{Na}_{2} \mathrm{HPO}_{4}$ \\
\hline & 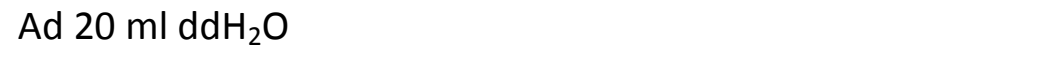 \\
\hline & $\mathrm{pH} 7.1$ \\
\hline 4x Resolving gel buffer & $0.4 \%$ SDS diluted in $1.5 \mathrm{M}$ Tris, $\mathrm{pH} 8.8$ \\
\hline $4 \times$ Stacking gel buffer & $0.4 \%$ SDS diluted in $0.5 \mathrm{M}$ Tris, $\mathrm{pH} 6.8$ \\
\hline \multirow[t]{6}{*}{$5 x$ Loading buffer } & 0.313 M Tris- $\mathrm{HCl}$ pH 6.8 \\
\hline & $10 \%$ SDS \\
\hline & 0.05\% Bromophenol blue \\
\hline & $50 \%$ Glycerol \\
\hline & 5 mM EDTA \\
\hline & $150 \mathrm{mM}$ DTT \\
\hline 5x Transfer buffer & $163 \mathrm{mM}$ Tris pH 8.3, 96 mM Glycine \\
\hline APS $(10 \% \mathrm{w} / \mathrm{v})$ & 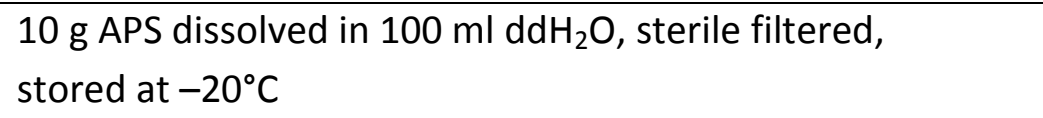 \\
\hline Cell lysis buffer & $\begin{array}{l}20 \mathrm{mM} \text { Tris- } \mathrm{HCl} \mathrm{pH} \mathrm{7.4,} 200 \mathrm{mM} \mathrm{NaCl} 20 \mathrm{mM} \mathrm{NaF} \text {, } \\
1 \% \text { IGEPAL CA-630, } 1 \mathrm{mM} \mathrm{Na}_{3} \mathrm{VO}_{4}, 1 \mathrm{mM} \mathrm{DTT}, 1 \text { tablet } \\
\text { PhosStop for } 10 \mathrm{ml}, 1 \text { tablet cOmplete (EDTA-free) for } 20 \mathrm{ml} \text {, } \\
\text { freshly prepared }\end{array}$ \\
\hline DAPI & $\begin{array}{l}\text { Stock solution: } 2 \mathrm{mg} / \mathrm{ml} \\
\text { Working solution: } 1: 50.000 \text { in } \mathrm{dd}_{2} \mathrm{O} \text {, } \\
\text { stored at } 4^{\circ} \mathrm{C} \text { under exclusion of light }\end{array}$ \\
\hline Enhanced & $4 \mathrm{ml}$ Solution SA \\
\hline luminescence (ECL) & $400 \mu \mathrm{l}$ Solution SB \\
\hline solution & $1.2 \mu \mathrm{l} 35 \% \mathrm{H}_{2} \mathrm{O}_{2}$ \\
\hline $\begin{array}{l}\text { Ficoll-loading-buffer } \\
(6 x)\end{array}$ & 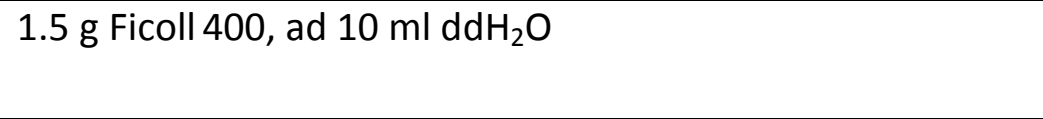 \\
\hline \multirow[t]{4}{*}{ Laemmli-buffer (10x) } & 864 g Glycine \\
\hline & $60 \mathrm{~g}$ SDS \\
\hline & $180 \mathrm{~g}$ Tris \\
\hline & Ad $6000 \mathrm{ml} \mathrm{ddH} \mathrm{H}_{2} \mathrm{O}$ \\
\hline
\end{tabular}




\begin{tabular}{|c|c|}
\hline \multirow[t]{6}{*}{ LB-medium } & $5 \mathrm{~g}$ Yeast extract \\
\hline & $10 \mathrm{~g} \mathrm{NaCl}$ \\
\hline & 10 g Trypton \\
\hline & 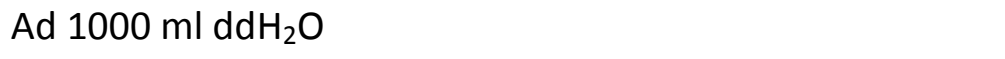 \\
\hline & $\mathrm{pH} 7.0$, autoclaved \\
\hline & For agar plates: $1.5 \%$ agar was added \\
\hline $\begin{array}{l}\text { Nonfat dry milk } \\
(5 \% \mathrm{w} / \mathrm{v})\end{array}$ & $5 \mathrm{~g}$ nonfat dry milk dissolved in $100 \mathrm{ml}$ 1x TBS-T buffer \\
\hline PFA (4\% w/v) & $4 \%$ PFA in DPBS, stored at $-20^{\circ} \mathrm{C}$ \\
\hline Resolving gel buffer & 1.5 M Tris, $\mathrm{pH} 8.8$ \\
\hline \multirow[t]{2}{*}{ SA solution } & 250 mg Luminol \\
\hline & ad $1000 \mathrm{ml} 0.1 \mathrm{M} \mathrm{Tris,} \mathrm{pH} 8.6$, storage at $4^{\circ} \mathrm{C}$ \\
\hline \multirow[t]{3}{*}{ SB solution } & 12 mg P-coumaric acid \\
\hline & ad $10 \mathrm{ml}$ DMSO, \\
\hline & stored at RT under exclusion of light \\
\hline SDS $(10 \% \mathrm{w} / \mathrm{v})$ & $10 \mathrm{~g}$ SDS dissolved in $100 \mathrm{ml}$, stored at RT \\
\hline \multirow[t]{4}{*}{ TBE buffer $(5 x)$} & $54 \mathrm{~g}$ Tris \\
\hline & $27.5 \mathrm{~g}$ Boric acid \\
\hline & $20 \mathrm{ml} 0.5 \mathrm{M}$ EDTA pH 8.0 \\
\hline & Ad $1 \mathrm{I} d d \mathrm{H}_{2} \mathrm{O}$ \\
\hline \multirow[t]{3}{*}{ TBS-T-buffer } & $20 \mathrm{mM}$ Tris \\
\hline & $150 \mathrm{mM} \mathrm{NaCl}$ \\
\hline & $0.1 \%$ Tween \\
\hline \multirow[t]{2}{*}{ Tyrode's solution } & $\mathrm{NaCl} 140 \mathrm{mM}, \mathrm{KCl} 5.4 \mathrm{mM}, \mathrm{CaCl}_{2} 1.8 \mathrm{mM}, \mathrm{MgCl}_{2} 1 \mathrm{mM}$, \\
\hline & HEPES 10 mM, Glucose 10 mM, pH 7.4 \\
\hline $0.1 \%$ Triton-X/BSA & $\begin{array}{l}10 \mu \mathrm{l} \text { Triton- } \mathrm{X} 100 \text { diluted in } 10 \mathrm{ml} 1 \% \text { BSA diluted in DPBS, } \\
\text { stored at } 4^{\circ} \mathrm{C}\end{array}$ \\
\hline
\end{tabular}

Table 12: List of competent E. coli cells

\begin{tabular}{lll}
\hline Competent cells & Genotype & Reference \\
\hline Top10 & F- mcrA $\Delta(m r r-h s d R M S-m c r B C)$ & Kindly provided by the \\
& Ф80lacZ $\Delta \mathrm{M} 15 \Delta / a c X 74$ recA1 & Department of Immunology, \\
& $\operatorname{araD139} \Delta($ ara leu $) 7697$ ga/U ga/K & University Medical Center \\
& $r p s L(S t r R)$ endA1 nupG & Göttingen, Thermo Fisher \\
& Scientific \\
\hline
\end{tabular}




\subsubsection{Chemicals, solutions, and media for cell culture}

Table 13: List of components for cell culture

\begin{tabular}{|c|c|}
\hline Components & Provider \\
\hline $0.25 \%$ Trypsin-EDTA & Thermo Fisher Scientific \#25200056 \\
\hline Albumin, human recombinant & Sigma-Aldrich \#A0237/A9731 \\
\hline B-27 serum free supplement (50x) & Thermo Fisher Scientific \#17504044 \\
\hline $\begin{array}{l}\text { Bovine albumin fraction } V \text { solution (BSA, } \\
7.5 \%)\end{array}$ & Thermo Fisher Scientific \#15260037 \\
\hline CHIR99021 & Merck Millipore \#361559 \\
\hline Collagenase B & Worthington Biochemical, \#CLS-AFB \\
\hline Collagenase IV & Worthington Biochemical \#CLS-4 \\
\hline CytoTune-iPS 2.0 Sendai reprogramming kit & $\begin{array}{l}\text { Thermo Fisher Scientific \#A16517, } \\
\text { A16518 }\end{array}$ \\
\hline $\begin{array}{l}\text { DMEM (Dulbecco's modified Eagle } \\
\text { medium)/F-12 }\end{array}$ & Thermo Fisher Scientific \#31331028 \\
\hline DMEM/F-12, no phenol red & Thermo Fisher Scientific \#21041025 \\
\hline DMEM & Thermo Fisher Scientific \#11960044 \\
\hline Essential 8 (E8) medium & Thermo Fisher Scientific \#A1517001 \\
\hline Fetal bovine serum (FBS) & Sigma-Aldrich \#F7524 \\
\hline Gelatin & Sigma-Aldrich \#48720 \\
\hline Geltrex & Thermo Fisher Scientific \#A1413301 \\
\hline HEPES sodium salt solution (1 M) & Sigma-Aldrich \#H3662 \\
\hline $\begin{array}{l}\text { Iscove's modified Dulbecco's medium } \\
\text { (IMDM) }\end{array}$ & Thermo Fisher Scientific \#31980022 \\
\hline IWP2 & Merck Millipore \#681671 \\
\hline Knockout serum replacement & Thermo Fisher Scientific \#10828028 \\
\hline L-ascobic acid 2-phosphate & Sigma-Aldrich \#A8960 \\
\hline L-glutamine (200 mM, 100x) & Thermo Fisher Scientific \#25030024 \\
\hline Mitomycin C & Serva Electrophoresis \#29805.02 \\
\hline Monothioglycerol (MTG) & Sigma-Aldrich \#M6145-25ML \\
\hline Non-essential amino acids (NEAA, 100x) & Thermo Fisher Scientific \#11140035 \\
\hline Penicillin-streptomycin solution (P/S) (100x) & Thermo Fisher Scientific \#15140122 \\
\hline Pro-survival compound & Merck Millipore \#529659 \\
\hline Recombinant human basic fibroblast & PeproTech \#100-18B \\
\hline
\end{tabular}




\begin{tabular}{|c|c|c|}
\hline \multicolumn{3}{|c|}{ growth factor (hbFGF) } \\
\hline \multicolumn{2}{|c|}{ RPMI 1640 with HEPES with GlutaMax } & Thermo Fisher Scientific \#72400021 \\
\hline \multicolumn{2}{|c|}{ RPMI 1640 without HEPES without Glucose } & Thermo Fisher Scientific \#11879020 \\
\hline \multicolumn{2}{|c|}{ Sodium DL-lactate solution $60 \%(w / w)$} & Sigma-Aldrich \#L4263 \\
\hline \multicolumn{2}{|c|}{ Thiazovivin (TZV) } & Millipore \# 420220 \\
\hline \multicolumn{2}{|c|}{ Trypsin-EDTA (0.25\%) } & Thermo Fisher Scientific \#25200056 \\
\hline \multicolumn{2}{|c|}{ Versene solution (0.48 mM EDTA) } & Thermo Fisher Scientific \#15040066 \\
\hline \multicolumn{2}{|c|}{$\beta$-Mercaptoethanol ( $\beta$-ME) } & Serva Electrophoresis \#28625 \\
\hline \multicolumn{3}{|c|}{ Human stem cell nucleofector kit $2 \quad$ Lonza \#VPH-5022 } \\
\hline \multicolumn{3}{|c|}{ Table 14: List of buffers and solutions for cell culture } \\
\hline Substance & \multicolumn{2}{|l|}{ Preparation } \\
\hline $100 \times \beta-M E$ & \multicolumn{2}{|c|}{$\begin{array}{l}0.07 \% \beta-M E \text { diluted in DPBS, sterile filtered, } \\
\text { stored at } 4^{\circ} \mathrm{C}\end{array}$} \\
\hline \multirow[t]{2}{*}{ Calyculin A } & \multicolumn{2}{|c|}{ Stock solution $10 \mu \mathrm{M}$ in $\mathrm{DMSO}$, stored at $-20^{\circ} \mathrm{C}$} \\
\hline & \multicolumn{2}{|c|}{ Working solution: $50 \mathrm{nM}$, diluted in RPMI1640 } \\
\hline $\mathrm{CHIR}(12 \mathrm{mM})$ & \multicolumn{2}{|c|}{$5 \mathrm{mg}$ CHIR99021 dissolved in $0.894 \mathrm{ml} \mathrm{DMSO}$, stored at $-20^{\circ} \mathrm{C}$} \\
\hline \multirow[t]{2}{*}{ Collagenase B } & \multicolumn{2}{|l|}{ Working solution: $400 \mathrm{U} / \mathrm{ml}$} \\
\hline & \multicolumn{2}{|c|}{ Dissolved in RPMI, sterile filtered, stored at $-20^{\circ} \mathrm{C}$} \\
\hline \multirow[t]{3}{*}{ Collagenase IV } & \multicolumn{2}{|l|}{ Stock solution: $2000 \mathrm{U} / \mathrm{ml}$} \\
\hline & \multicolumn{2}{|l|}{ Working solution: $200 \mathrm{U} / \mathrm{ml}$} \\
\hline & \multicolumn{2}{|c|}{ Dissolved in DMEM/F12, sterile filtered, stored at $-20^{\circ} \mathrm{C}$} \\
\hline Gelatin (0.1\%) & \multicolumn{2}{|c|}{ 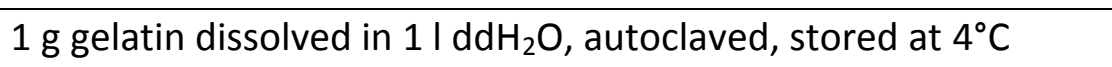 } \\
\hline Geltrex & \multicolumn{2}{|c|}{$2 \mathrm{mg}$ Geltrex per $15 \mathrm{ml}$ falcon tube, stored at $-20^{\circ} \mathrm{C}$} \\
\hline \multirow[t]{3}{*}{ hbFGF } & \multicolumn{2}{|l|}{ Stock solution: $100 \mathrm{ng} / \mu \mathrm{l}$} \\
\hline & \multicolumn{2}{|c|}{$100 \mu \mathrm{g}$ hbFGF dissolved in $1 \mathrm{ml}$ Tris $(5 \mathrm{mM})$, stored at $-20^{\circ} \mathrm{C}$} \\
\hline & \multicolumn{2}{|c|}{ Working solution: $5 \mathrm{ng} / \mu \mathrm{l}$, diluted $1: 20$ in $0.1 \% \mathrm{BSA}$, stored at $4^{\circ} \mathrm{C}$} \\
\hline Isoprenaline & Stock solution: $1 \mathrm{mM}$ & \\
\hline & Dissolved in $\mathrm{dd}_{2} \mathrm{O}$, stored & $\mathrm{t}-20^{\circ} \mathrm{C}$ \\
\hline & Working solution: $100 \mathrm{nM}$, & liluted in RPMI1640 \\
\hline IWP2 (5 mM) & $\begin{array}{l}10 \mathrm{mg} \text { dissolved in } 4.28 \mathrm{ml} \\
\text { incubated for } 10 \text { minutes a }\end{array}$ & $\begin{array}{l}\mathrm{MSO} \text {, } \\
37^{\circ} \mathrm{C} \text {, stored at }-20^{\circ} \mathrm{C}\end{array}$ \\
\hline L-755,507 & Stock solution: $5 \mathrm{mM}$, disso & ved in DMSO \\
\hline & Working solution: $5 \mu \mathrm{M}$ & \\
\hline MTG & Stock solution: $150 \mathrm{mM}$ & \\
\hline & $13 \mu \mathrm{l}$ MTG diluted in $1 \mathrm{ml} \mathrm{In}$ & IDM, sterile filtered, freshly prepared \\
\hline TZV (2 mM) & $10 \mathrm{mg}$ TZV dissolved in 6.8 & l DMSO, stored at $-20^{\circ} \mathrm{C}$ \\
\hline
\end{tabular}


Table 15: List of media for human cell lines

\begin{tabular}{|c|c|}
\hline Medium & Components \\
\hline \multirow[t]{2}{*}{ B27 medium } & $500 \mathrm{ml}$ RPMI 1640 with HEPES \\
\hline & 1x B27 supplement with insulin \\
\hline Cardio differentiation & $500 \mathrm{ml}$ RPMI 1640 with HEPES with GlutaMAX \\
\hline \multirow[t]{2}{*}{ medium } & 250 mg Albumin, human recombinant \\
\hline & $100 \mathrm{mg}$ L-Ascorbic acid 2-phosphate, sterile filtered \\
\hline \multirow[t]{3}{*}{ Cardio digestion medium } & $80 \mathrm{ml} \mathrm{B27}$ medium \\
\hline & $20 \mathrm{ml} \mathrm{FBS}$ \\
\hline & $100 \mu \mathrm{lTZV}$ \\
\hline $\begin{array}{l}\text { CRISPR/Cas9 freezing } \\
\text { medium }\end{array}$ & E8 medium, 10\% DMSO, $2 \mu \mathrm{M}$ TZV \\
\hline E8 freezing medium & E8 medium, 20\% DMSO, $4 \mu \mathrm{M}$ TZV \\
\hline \multirow[t]{3}{*}{ FACS medium } & 1x DMEM/F-12, no phenol red \\
\hline & $1 \times \mathrm{P} / \mathrm{S}, 1.5 \%$ heat-inactivated $\mathrm{FBS}$, \\
\hline & $4 \mu \mathrm{M}$ TZV, $10 \mathrm{ng} / \mathrm{ml}$ hbFGF \\
\hline FL-medium & DMEM, $15 \%$ heat-inactivated FBS, $1 \times$ L-Glutamine \\
\hline \multirow[t]{3}{*}{ hES freezing medium } & $18 \mathrm{ml}$ DMEM \\
\hline & $5 \mathrm{ml} \mathrm{FBS}$ \\
\hline & $2 \mathrm{ml}$ DMSO \\
\hline \multirow[t]{5}{*}{ hES medium } & DMEM/F12, GlutaMAX \\
\hline & $15 \%$ Knock out serum replacement \\
\hline & $1 \times$ NEAA \\
\hline & $1 \times \beta-M E$ \\
\hline & $10 \mathrm{ng} / \mathrm{ml} \mathrm{hbFGF}$ \\
\hline \multirow[t]{5}{*}{ HFBM } & DMEM \\
\hline & $10 \%$ heat-inactivated FBS \\
\hline & $1 \times$ NEAA \\
\hline & $1 \times \beta-M E$ \\
\hline & $10 \mathrm{ng} / \mathrm{ml} \mathrm{hbFGF}$ \\
\hline \multirow[t]{2}{*}{ E8 medium } & $500 \mathrm{ml}$ E8 basal medium \\
\hline & 1x E8 Supplement \\
\hline \multirow[t]{4}{*}{ Iscove medium } & IMDM, GlutaMAX \\
\hline & $20 \%$ heat-inactivated FBS \\
\hline & $1 \times$ NEAA \\
\hline & $450 \mu \mathrm{M}$ MTG (freshly added) \\
\hline $\begin{array}{l}\text { MEF-conditioned E8 } \\
\text { medium }\end{array}$ & $\begin{array}{l}\text { E8 medium was incubated for } 24 \text { hours on mitomycin C- } \\
\text { treated MEFs }\end{array}$ \\
\hline
\end{tabular}




\subsubsection{Cells used in the study}

Mouse embryonic fibroblasts (MEFs): isolated from 15- to 17-day-old embryos of NMRI mice (Central Animal Facility, University Medical Center Göttingen)

Human embryonic kidney 293T cells (HEK293T): used for the transient expression of CRISPR/Cas9 plasmids (University Medical Center Göttingen)

Human fibroblasts: isolated from human skin biopsies of healthy subjects and CPVT patients.

Control hiPSCs (Ctrl-hiPSCs): generated with STEMCCA lentivirus or SeV from human fibroblasts, blood and mesenchymal stem cells of healthy subjects (WTD2, C113, BM76.3, WT1bld and WT4bld) without known cardiac symptoms (University Medical Center Göttingen) (Table 16).

CPVT-hiPSCs: generated from human skin fibroblasts of CPVT patients (CPVTa2, CPVTa3, CPVTb1, CPVTc1, CPVTc2, CPVTd1) carrying different RYR2 point mutations (University Medical Center Göttingen) (Table 16). HiPSCs from the patient CPVTb1, CPVTc1 and CPVTC2 were generated within this study.

Table 16: hiPSC lines used in this work and their specifications

\begin{tabular}{lllll}
\hline $\begin{array}{l}\text { Patient/Subject/ } \\
\text { Cell line }\end{array}$ & Mutation & Donor & Cell line origin & $\begin{array}{l}\text { Reprogramming } \\
\text { virus }\end{array}$ \\
\hline WTD2 & Healthy & - & Skin fibroblasts & STEMCCA \\
\hline C113 & Healthy & - & Skin fibroblasts & STEMCCA \\
\hline BM76.3 & Healthy & Male & $\begin{array}{l}\text { Mesenchymal } \\
\text { stem cells }\end{array}$ & STEMCCA \\
\hline isWT1bld & Healthy & Male & Blood & SeV \\
\hline isWT4bld & Healthy & Male & Blood & SeV \\
\hline isCPVTa2 & R420W & Female & Skin fibroblasts & SeV \\
\hline isCPVTa3 & R420W & Female & Skin fibroblasts & SeV \\
\hline $\begin{array}{l}\text { isCPVTb1/ } \\
\text { CPVTb1 }\end{array}$ & A2254V & Male & Skin fibroblasts & SeV / STEMCCA \\
\hline $\begin{array}{llll}\text { isCPVTc1/ } \\
\text { CPVTc1 }\end{array}$ & E4076K & Female & Skin fibroblasts & SeV/ STEMCCA \\
\hline $\begin{array}{l}\text { isCPVTc2/ } \\
\text { CPVTc2 }\end{array}$ & E4076K & Female & Skin fibroblasts & SeV/ STEMCCA \\
\hline isCPVTd1 & H4742Y & Female & Skin fibroblasts & SeV \\
\hline
\end{tabular}




\subsubsection{Software}

Table 17: List of software used for analysis

\begin{tabular}{lll}
\hline Name & Purpose & Company/Author \\
\hline Adobe Photoshop & Image processing & Adobe \\
\hline GENtle & Sequencing analysis & Markus Manske \\
\hline ImageJ & Image processing & National Institutes of Health \\
\hline Graphpad Prism 6 & Statistical data analysis and & Graphpad Software, Inc. \\
& graph design & \\
\hline AxioVision & Immunofluorescence images & Carl Zeiss \\
\hline Zen & Confocal images & Carl Zeiss \\
\hline Alpha.Imager & Calculation of CRISPR/Cas9 & Alpha-Imager \\
Software & cleavage efficacy & \\
\hline
\end{tabular}

\subsection{Methods}

\subsubsection{Cell biological methods}

All cells were cultivated under humidified conditions at $37^{\circ} \mathrm{C}$ and $5 \%$ carbon dioxide. All culture work was performed under sterile conditions using a laminar airflow cabinet to avoid microbiological contamination.

\subsubsection{Counting of cells}

For counting of cells, approx. $20 \mu \mathrm{l}$ of the cell suspension was analyzed using a Thoma hemocytometer and a transmitted light microscope. Cells in a grid of two $4 \times 4$ quadrants were counted and the total cell number per $\mathrm{ml}$ was calculated using the following formula: mean value of counted cells $/ 64 * 10^{6}$.

\subsubsection{Cultivation and inactivation of mouse embryonic fibroblasts}

MEFs were isolated from 15- to 17-day-old NMRI mouse embryos and subsequently cultivated on $0.1 \%$ gelatin-coated culture dishes in FL-medium for maximal 4 passages. Before used for cultivation of hiPSCs, MEFs were treated with a final concentration of $10 \mathrm{\mu g} / \mathrm{ml}$ mitomycin $\mathrm{C}$ for $3 \mathrm{~h}$ at $37^{\circ} \mathrm{C}$ to stop cell proliferation. MEFs were washed three times with DPBS and subsequently treated with $0.25 \%$ Trypsin-EDTA until cells started to detach. Single cells were resuspended in FL-medium and counted. $1.2 \times 10^{5}$ cells were then plated onto $0.1 \%$ gelatin coated 6 -cm TC-treated culture dishes. 


\subsubsection{Isolation and cultivation of human fibroblasts}

For isolation of human skin fibroblasts, skin punch biopsy (3.5-4 mm) was taken aseptically by a surgeon, transferred in a sterile container with DMEM plus $2 x \mathrm{P} / \mathrm{S}$ and transported as soon as possible to the laboratory. Tissue was rinsed with warm DPBS to remove excess blood and transferred to the lid of the petri dish. The fat tissue was removed using a scalpel and forceps, and the skin biopsy was cut into small pieces (about $0.5 \mathrm{~mm}$ to $1 \mathrm{~mm}$ in size). The small pieces of tissue were transferred onto $6-\mathrm{cm} \mathrm{TC}$ treated dishes without medium, pressed carefully on the dish and cultured in HFBM plus $2 x \mathrm{P} / \mathrm{S}$. Medium was changed the third day after plating the tissue pieces. Fibroblasts started to grow out at 7-10 days after plating, and P/S was omitted after 10 days. After two weeks in culture, fibroblasts derived from skin biopsies were passaged for the first time. To this end, pieces of tissue were transferred to a TC-treated dish containing HFBM for further cultivation. Medium was aspirated and prewarmed $0.25 \%$ Trypsin-EDTA was incubated for $1 \mathrm{~min}$ at RT. Trypsin-EDTA was removed and cells were resuspended in HFBM and transferred to a new plate. Fibroblasts were passaged with $0.25 \%$ TrypsinEDTA when confluent and used for transduction experiments before passage 3-4.

\subsubsection{Generation of hiPSCs using CytoTune-iPS 2.0 Sendai reprogramming kit}

For the reprogramming with $\mathrm{SeV}$, early passage fibroblasts (p2-p3) were used. To this end, fibroblasts were washed twice with DMEM and detached with 0.25\% Trypsin-EDTA as described in 2.2.1.3. $8 \times 10^{4}$ singularized cells were plated into one well of a TC-treated 6-well plate containing HFBM. After 1-2 days cell density reached $50-80 \%$ and cells were used for transduction. CytoTune2.0 Sendai virus cocktail was prepared based on the counted cell number (approximately $1 \times 10^{5}$ cells per well) according to manufacturer's protocol for $\mathrm{MOI}$ and the mixed virus cocktail was transferred into $0.5 \mathrm{ml} \mathrm{HFBM}$. For each well, $0.5 \mathrm{ml}$ virus containing medium was added into each well containing $1 \mathrm{ml} \mathrm{HFBM}$ plus $1 \times \mathrm{P} / \mathrm{S}$ and mixed gently by pipetting. Virus was incubated for 24 hours and medium was changed daily until day 6 . At day 7 , fibroblasts were singularized using $0.25 \%$ TrypsinEDTA as previously described and $2-8 \times 10^{5}$ cells were plated on MEF-coated TC-treated 6well plates containing hES-medium plus hbFGF $(20 \mathrm{ng} / \mathrm{ml})$. Medium was changed daily. First colonies appeared 2-3 weeks after transduction and were picked manually under sterile conditions using light microscopy and glass pipettes. Single colonies were transferred into MEF-coated 12-well plates containing hES medium plus $20 \mathrm{ng} / \mathrm{ml} \mathrm{hbFGF}$ and $1 \mathrm{x} / \mathrm{S}$. Medium was changed daily with $\mathrm{hES}$ medium plus $20 \mathrm{ng} / \mathrm{ml} \mathrm{hbFGF}$. After passaging, cells were further cultivated as described in 2.2.1.5. 


\subsubsection{Cultivation of hiPSCs with feeder cells}

For the pluripotency characterization of hiPSCs, undifferentiated hiPSCs were cultivated on mitomycin C-treated MEFs (see 2.2.1.2) in hES medium. The medium was changed daily and cells were split every 4 to 5 days. The cells were treated with collagenase IV $\left(200 \mathrm{U} / \mathrm{ml}, 5 \mathrm{~min}, 37^{\circ} \mathrm{C}\right.$ ) and washed twice with DMEM/F-12. The cells were cut into small clusters using a cell scraper, gently resuspended and transferred onto new culture dishes with inactivated MEFs. Differentiated colonies were removed mechanically by suction.

\subsubsection{Preparation of Geltrex-coated dishes and glass coverslips}

Geltrex was thawed on ice, aliquoted in $2 \mathrm{mg}$ portions and stored at $-20^{\circ} \mathrm{C}$. Geltrex was resuspended with cold DMEM/F-12 and distributed in culture plates to a final concentration of $17 \mu \mathrm{g} / \mathrm{cm}^{2}$ (per well of a 6 -well plate) or $21 \mu \mathrm{g} / \mathrm{cm}^{2}$ (per well of 12 -well plate). Cell culture plates were incubated for $30 \mathrm{~min}$ at $37^{\circ} \mathrm{C}$ or overnight at $4^{\circ} \mathrm{C}$. For preparation of glass coverslips, glass slips $(\varnothing 25 \mathrm{~mm})$ were incubated in $0.1 \% \mathrm{HCl}$ overnight and transferred into $70 \%$ ethanol for 12 hours. Afterwards, slips were dried and heated up for sterilization at $200^{\circ} \mathrm{C}$ for $2 \mathrm{~h}$. Coverslips were placed into 6-well plates and coated with Geltrex as described above.

\subsubsection{Transfer of hiPSCs from feeder layer onto Geltrex-coated dish}

After at least 8-10 passages, hiPSCs cultured on feeder layer were adapted to feeder-free cultures using Geltrex as coating matrix which contains mainly laminin, collagen IV, entactin, and heparin sulfate proteoglycan. To this end, medium from hiPSC culture on feeder layer was aspirated. The cells were washed twice and incubated for 3-4 min with Versene at RT. After aspirating Versene carefully, cells were detached by resuspending with E8 medium and plated at high density (1:4) onto Geltrex-coated dishes. Medium was changed daily with E8 medium.

\subsubsection{Cultivation of hiPSCs on Geltrex}

At about $85-90 \%$ confluence, hiPSCs were passaged onto new plates. Cells were washed twice and incubated for 3 to $4 \mathrm{~min}$ with Versene at RT. The small cell clusters or rather single cells were subsequently transferred onto new Geltrex-coated plates containing E8 medium supplemented with $2 \mu \mathrm{M} \mathrm{TZV}$. TZV was removed after 24 hours and the medium was changed daily. HiPSCs were cultured up to passage 60. After 4-5 passages on Geltrex, cells could be used for cardiac differentiation experiments (2.2.1.10). 


\subsubsection{Spontaneous differentiation of hiPSCs in vitro}

For spontaneous in vitro differentiation experiments, hiPSCs cultured on feeder cells were treated with $200 \mathrm{U} / \mathrm{ml}$ collagenase IV (see 2.2.1.5) and dissected with a cell scraper into big cell clusters. Cell clusters were transferred to untreated $6-\mathrm{cm}$ dishes and kept in suspension with hES medium and incubated for 24 hours (day 0). During this time, the cells formed multi-cellular aggregates known as embryoid bodies (EBs). The medium was changed to Iscove medium and changed every second day. At day 8 , EBs were plated on $0.1 \%$ gelatin-coated TC-treated 6 -cm dishes with or without glass coverslips (18 $\mathrm{mm} \times 18$ $\mathrm{mm}$ ). During this spontaneous differentiation, clusters of beating CMs and other cell types appeared. For reverse transcription-PCR analysis of tissue-specific markers, pellets were taken at day $8+3 / 8+17$ and day $8+25$ as described in 2.2.1.13. For immunofluorescence analysis, cells were stained for the endodermal marker AFP at day $8+3 / 8+17$. The mesodermal marker $\alpha$-SMA and the ectodermal marker $\beta$-III-TUBULIN were stained at day $8+25$.

\subsubsection{Directed differentiation of hiPSCs into cardiomyocytes}

For direct differentiation of hiPSCs into CMs, hiPSCs cultured on Geltrex were transferred into 12-well plates and homogeneously distributed. When cells reached about $80-90 \%$ confluence, differentiation was initiated by cultivation in Cardio differentiation medium supplemented with $4 \mu \mathrm{M}$ CHIR99021, a glycogen synthase kinase 3 inhibitor that induces activation of WNT signaling (day 0 ). Medium was replaced after 48 hours by Cardio differentiation medium supplemented with $5 \mu \mathrm{M}$ IWP2, a WNT pathway inhibitor (day 2) and changed again after 48 hours with Cardio differentiation medium. At day 6, medium was replaced by B27 medium. Medium was changed every 3-4 days with B27 medium. First beating CMs were observed between 8-11 days. To achieve CM maturation, CMs were further cultivated for three months.

\subsubsection{Digestion and selection of iPSC-derived CMs}

For detachment of beating $\mathrm{CMs}$, cells were gently digested with collagenase $\mathrm{B}(400 \mathrm{U} / \mathrm{ml}$ in RPMI1640) for $30-40 \mathrm{~min}$ at $37^{\circ} \mathrm{C}$. Detached cells were collected in a falcon tube, centrifuged for $5 \mathrm{~min}$ at $200 \times \mathrm{g}$ and carefully resuspended with $0.25 \%$ Trypsin-EDTA (5 min, $37^{\circ} \mathrm{C}$ ). Trypsinization was stopped with Cardio digestion medium and singularized cells were again centrifuged and re-suspended in Cardio digestion medium. About $1 \times 10^{6}$ cells were seeded in a Geltrex-coated well of a 6-well plate containing Cardio digestion medium. After 24 hours, medium was changed to B27 medium to remove FBS and TZV. 
Once cells had recovered from digestion and spontaneous contraction had re-started (around day 15), cells were put under selective pressure to purify the CM population. Cells were cultured for 4-6 days in Cardio selection medium containing lactate instead of glucose. From here on CMs were cultivated in B27 medium until maturity levels had been reached.

\subsubsection{Freezing and thawing of cultivated cells}

For cryopreservation, hiPSCs cultured on feeder layer were treated with collagenase IV and dissected into small pieces as described in 2.2.1.5. Cell clusters were transferred into $15 \mathrm{ml} \mathrm{DMEM} / \mathrm{F}-12$ and centrifuged ( $200 \times \mathrm{g}, 5 \mathrm{~min}$ ). The supernatant was discarded and the cells were carefully resuspended in $1 \mathrm{ml}$ hES freezing medium and transferred into cryo tubes.

For cryopreservation of hiPSCs cultured on Geltrex, cells were washed twice with Versene and incubated for $5 \mathrm{~min}$. Versene was aspirated carefully and cells were detached by adding $0.75 \mathrm{ml}$ E8 medium. After resuspending, $0.75 \mathrm{ml}$ E8 freezing medium was added. The suspension was mixed gently and the total $1.5 \mathrm{ml}$ was transferred in a cryo tube immediately. The cells were kept overnight at $-80^{\circ} \mathrm{C}$ in a freezing box containing isopropanol, allowing controlled freezing at $-1^{\circ} \mathrm{C}$ per min. The following day, the frozen cells were stored in liquid nitrogen.

For thawing, frozen cells were placed in a $37^{\circ} \mathrm{C}$ warm water bath. Immediately after thawing, cells were transferred into $10 \mathrm{ml}$ cold DMEM/F-12 and centrifuged (200 $\times \mathrm{g}$, $5 \mathrm{~min}$ ). The supernatant was discarded and the cell pellet was resuspended in hES or E8 medium and transferred onto 6-cm or 6-well TC-treated culture dishes either coated with inactivated MEFs or with Geltrex, respectively.

\subsubsection{Harvesting of cells for pellets}

Cells were washed twice with DPBS, covered with $1.5 \mathrm{ml}$ DPBS and harvested by using a cell scraper. Detached cells were transferred into a $1.5 \mathrm{ml}$ reaction tube and centrifuged one minute at $16,000 \times \mathrm{g}$. The supernatant was removed and the reaction tube was flash frozen in liquid nitrogen and stored at $-80^{\circ} \mathrm{C}$ until analysis.

\subsubsection{Alkaline phosphatase staining}

One of the first evidence for successful somatic cell reprogramming is alkaline phosphatase activity. In reprogrammed cells, alkaline phosphatase expression is significantly increased. Alkaline phosphatase activity in hiPSCs was detected using an 
alkaline phosphatase staining kit according to the manufacturer's instructions. Cells were washed with DPBS and subsequently fixed for $30 \mathrm{sec}$ using a fixation solution provided by the kit. Fixed cells were washed twice with $\mathrm{dd}_{2} \mathrm{O}$ and stained for $15 \mathrm{~min}$ at $37^{\circ} \mathrm{C}$ using a staining solution. After a final washing step with $\mathrm{ddH}_{2} \mathrm{O}$, cells were dried at RT. Alkaline phosphatase positive cells are stained in red.

\subsubsection{Teratoma formation and analyses}

Undifferentiated hiPSCs cultured on feeder layer were mechanically dissected using a cell scraper and transferred into 200-300 $\mu$ DPBS. The cell clusters were injected

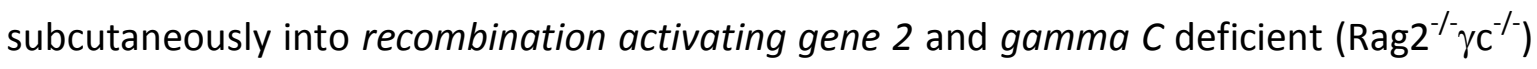
mice, which lack B cells, T cells, and natural killer cells. Teratomas were collected about three months after injection and fixed in phosphate buffered formalin ( $\mathrm{pH} 7.0)$ for 4 hours at RT or overnight at $4^{\circ} \mathrm{C}$. After washing with $\mathrm{dd}_{2} \mathrm{O}$, teratomas were dehydrated. The samples were embedded into paraffin using a tissue embedding system and processed into $6 \mu \mathrm{m}$ sections with a microtome. The injection of hiPSCs into Rag $2^{-/-} \mathrm{Cc}^{-/-}$mice as well as the section of teratomas were performed by technicians in Dr. Guan's lab. Histological sections were stained with hematoxylin and eosin at the Department of Pathology of the University Medical Center Göttingen and subsequently analyzed using light microscopy.

\subsubsection{Molecular biological analysis}

\subsubsection{RNA isolation}

For gene expression analyses, cultured cells were washed three times with DPBS and pelleted (see 2.2.1.13). Depending on the density, cells were lysed with 300-500 $\mu$ l RNA lysis buffer. Total RNA isolation and purification was done with the SV total RNA isolation system according to the manufacturer's instructions without the heating step in RNA dilution buffer. Elution was performed using 60-100 $\mu \mathrm{l}$ nuclease-free $\mathrm{H}_{2} \mathrm{O}$. The concentration of the isolated RNA was measured with the NanoDrop 2000c/2000 at $260 \mathrm{~nm}$ and $280 \mathrm{~nm}$. The RNA was subsequently used for reverse transcription reaction or stored at $-80^{\circ} \mathrm{C}$.

\subsubsection{Reverse transcription reaction}

After RNA isolation, total mRNA was transcribed into complementary DNA (cDNA) using the enzyme reverse transcriptase. All components for one reverse transcription reaction are listed in Table 18. 
Table 18: List of components for reverse transcription reaction

\begin{tabular}{|c|c|}
\hline Components & $20 \mu \mathrm{l}$ final volume \\
\hline 10x PCR buffer II & $2 \mu \mathrm{l}$ \\
\hline $25 \mathrm{mM} \mathrm{MgCl}_{2}$ & $4 \mu \mathrm{l}$ \\
\hline $100 \mathrm{mM}$ dNTPs & $0.8 \mu \mathrm{l}$ \\
\hline RNase inhibitor $(20 \mathrm{U} / \mu \mathrm{l})$ & $1 \mu \mathrm{l}$ \\
\hline $50 \mu \mathrm{M}$ Oligo $d(T) 16$ & $1 \mu l$ \\
\hline MuLV Reverse transcriptase $(50 \mathrm{U} / \mu \mathrm{l})$ & $1 \mu \mathrm{l}$ \\
\hline $100-250$ ng RNA and nuclease-free $\mathrm{H}_{2} \mathrm{O}$ & $10.2 \mu \mathrm{l}$ \\
\hline
\end{tabular}

The reverse transcription reaction was accomplished in a thermocycler using the following program:

\begin{tabular}{ll}
\hline Temperature & time \\
\hline $22^{\circ} \mathrm{C}$ & $10 \mathrm{~min}$ \\
\hline $42^{\circ} \mathrm{C}$ & $50 \mathrm{~min}$ \\
\hline $95^{\circ} \mathrm{C}$ & $10 \mathrm{~min}$ \\
\hline $4^{\circ} \mathrm{C}$ & $\infty$ \\
\hline
\end{tabular}

The resulting cDNA was stored at $-20^{\circ} \mathrm{C}$

\subsubsection{Polymerase chain reaction}

All components for one PCR to amplify certain CDNA fragments are listed in Table 19.

Table 19: List of components for PCR

\begin{tabular}{ll}
\hline Components for PCR & $\mathbf{2 5} \boldsymbol{\mu l}$ final volume \\
\hline cDNA & $1 \mu \mathrm{l}$ \\
\hline Nuclease-free $\mathrm{H}_{2} \mathrm{O}$ & $15.3 \mu \mathrm{l}$ \\
\hline $5 \times$ Green GoTaq reaction buffer & $5 \mu \mathrm{l}$ \\
\hline $10 \mathrm{mM}$ dNTPs & $1.6 \mu \mathrm{l}$ \\
\hline Sense primer $(10 \mu \mathrm{M})$ & $1 \mu \mathrm{l}$ \\
\hline Antisense primer $(10 \mu \mathrm{M})$ & $1 \mu \mathrm{l}$ \\
\hline GoTaq G2 DNA polymerase $(5 \mathrm{U} / \mu \mathrm{l})$ & $0.1 \mu \mathrm{l}$
\end{tabular}

The reaction was performed in a thermocycler using the following standardized program: 


\begin{tabular}{l|l|l|l}
\hline \multicolumn{1}{c}{ Step } & \multicolumn{2}{c}{ Temperature } & \multicolumn{2}{c}{ Time } & \multicolumn{1}{c}{ Repeats } \\
\hline \multirow{2}{*}{ Denaturation } & $95^{\circ} \mathrm{C}$ & $3 \mathrm{~min}$ & 1 \\
\cline { 2 - 3 } & $94^{\circ} \mathrm{C}$ & $15 \mathrm{sec}$ & $28-45$ \\
\hline Annealing* & $50-65^{\circ} \mathrm{C}$ & $30 \mathrm{sec}$ & \\
\hline Elongation & $72^{\circ} \mathrm{C}$ & $30 \mathrm{sec}$ & \\
\cline { 3 - 4 } & & $10 \mathrm{~min}$ & 1 \\
\hline Final step & $4{ }^{\circ} \mathrm{C}$ & $\infty$ & 1 \\
\hline
\end{tabular}

*Exact annealing temperature and number of cycles for the different oligonucleotides are listed in Tables 4 and 6.

\subsubsection{Agarose gel electrophoresis}

Amplified PCR products were analyzed by electrophoretic separation on an 1.5$2 \%$ agarose gel. The agarose gel was prepared using appropriate amounts of agarose, $1 x$ TBE buffer and Midori Green Advance $(0.04 \mu \mathrm{l} / \mathrm{ml})$ for the visualization of DNA. The electrophoresis was performed at $120 \mathrm{~V}$ for approx. $45 \mathrm{~min}$. The particular size of the DNA fragments was determined by GeneRuler 100 bp Plus DNA Ladder. All results were documented using ultraviolet light.

\subsubsection{Purification of DNA fragments from PCR}

DNA purification of PCR products and DNA extraction from agarose gels were performed using QIAquick PCR purification kit and QIAquick gel extraction kit, respectively, according to manufacturer's protocols. Elution was performed using $30 \mu \mathrm{l}$ nuclease-free $\mathrm{H}_{2} \mathrm{O}$.

\subsubsection{Heat-shock transformation of chemo-competent E. coli}

For the amplification of CRISPR/Cas9-plasmids, plasmid-DNA was transformed into E. coli Top10F cells. To this end, competent E. coli were thawed on ice and mixed with $20 \mathrm{ng}$ pure plasmid-DNA. Cells were gently mixed by vortexing and incubated for 20 min on ice. The following heat-shock was performed at $42^{\circ} \mathrm{C}$ for $90 \mathrm{sec}$ in a thermomixer. Afterwards the cells were incubated on ice for additional $2 \mathrm{~min}$, resuspended in $500 \mu \mathrm{LB}$ medium (without antibiotics) and incubated for $60 \mathrm{~min}$ at $37^{\circ} \mathrm{C}$ in a thermomixer. 50 and $100 \mu \mathrm{l}$ of the cell suspension was directly plated on LB-agar plates with kanamycin $(50 \mu \mathrm{g} / \mathrm{ml})$. The plates were incubated at $37^{\circ} \mathrm{C}$ overnight. 


\subsubsection{Plasmid purification from E. coli cultures}

Plasmid-harboring E. coli cultures were pre-cultured in $6 \mathrm{ml} \mathrm{LB}$-medium with kanamycin $\left(50 \mu \mathrm{g} / \mathrm{ml}\right.$ ) for 6 hours at $37^{\circ} \mathrm{C} .2 \mathrm{ml}$ of this E. coli culture were used to inoculate $250 \mathrm{ml} \mathrm{LB}$ medium with kanamycin, which was incubated at $37^{\circ} \mathrm{C}$ overnight in a shaking incubator. Afterwards, $800 \mu \mathrm{l}$ of this culture was used for a glycerin stock (200 $\mu$ l 99\% glycerin and $800 \mu \mathrm{l}$ of $\mathrm{E}$. coli culture). The isolation of plasmid DNA was done by the principle of alkaline lysis. To this end, the QIAGEN plasmid maxi kit was used according to the manufacturer's instructions. The plasmid-DNA-pellet was resuspended with 150-200 $\mu \mathrm{l}$ sterile nuclease-free $\mathrm{H}_{2} \mathrm{O}$.

\subsubsection{Genomic DNA isolation for DNA sequencing}

Prior to genomic DNA isolation, cultivated cells were detached from the plates as described in 2.2.1.13 and pellets were stored at $-80^{\circ} \mathrm{C}$. The genomic DNA was isolated and purified using the automated Maxwell 16 cell DNA purification kit according to the manufacturer's instructions. The concentration of the isolated DNA was measured using a NanoDrop at $260 \mathrm{~nm}$ and $280 \mathrm{~nm}$. The samples were subsequently stored at $-80^{\circ} \mathrm{C}$. For genomic DNA sequencing, the DNA sequence from the gene of interest (RYR2) was initially amplified by PCR using the appropriate primer set. In each reaction, $100 \mathrm{ng}$ of total genomic DNA was used. The PCR product was electrophoretically separated on an 1.5\% agarose gel and subsequently excised with a scalpel. The DNA fragment was then extracted from the agarose gel using the QIAquick gel extraction kit according to the manufacturer's instructions.

\subsubsection{DNA sequencing}

DNA Sequencing of PCR products from CPVT patients and CRISPR clones was performed by a commercial sequencing facility (Seqlab, Göttingen). To this end, 200 ng DNA was mixed with $3 \mu$ l sequencing-primer $(10 \mu \mathrm{M})$ and adjusted with nuclease-free $\mathrm{H}_{2} \mathrm{O}$ to a total volume of $15 \mu \mathrm{l}$.

\subsubsection{Allele-specific expression analysis of the $R Y R 2$ gene}

CPVT patients are characterized by a heterogeneous point mutation in the $R Y R 2$ gene. For an allele-specific expression analysis of $R Y R 2$, the cDNA of differentiated three-month-old CMs was used. To this end, the total amount of mRNA was isolated and $250 \mathrm{ng}$ RNA were reverse transcribed into cDNA as described before (see 2.2.4.2 and 2.2.4.3). DNA fragments (267-289 bp) which include the region of the point mutation were amplified as 
described in Table 20. Primer sets are listed in Table 7. The PCR product of two $50 \mu \mathrm{I}$ PCR reactions was purified using the QIAquick PCR purification kit according to the manufacturer's instructions and eluted in $30 \mu \mathrm{l}$ nuclease-free $\mathrm{H}_{2} \mathrm{O}$. Sequencing of cDNA copies was done in the Transcriptome and Genome Analysis Laboratory of the University Medical Center Göttingen. For sequencing, the cDNA samples were prepared with the "TruSeq RNA Sample Prep Kit v2" (Illumina ${ }^{\circledR}$ ) according to the manufacturer's protocols. Paired-end ( $2 \times 250 \mathrm{bp}$ ) sequencing was conducted using a MiSeq (Illumina ${ }^{\circledR}$ ).

Sequencing quality was checked and approved via the FastQC software (http://www.bioinformatics.babraham.ac.uk/projects/fastqc/). Sequences were aligned to the genome reference sequence of Homo sapiens (Ensembl assembly GRCh38) using the STAR alignment software (Pubmed ID_23104886; version 2.5) with standard parameters. Optical and PCR duplicates were removed using Picard tools (http://broadinstitute.github.io/picard/, version 2.3.0). Variant calling, filtering and allelespecific expression quantification were conducted using GATK tools according to best practices (Pubmed ID_21478889, version 3.4.46).

Table 20: PCR for the amplification of CDNA used for allele-specific expression analysis

\begin{tabular}{ll}
\hline Components for PCR & $\mathbf{5 0} \boldsymbol{\mu l}$ final volume \\
\hline cDNA & $8 \mu \mathrm{l}$ \\
\hline Nuclease-free $\mathrm{H}_{2} \mathrm{O}$ & $24.6 \mu \mathrm{l}$ \\
\hline $5 x$ Green GoTaq reaction buffer & $10 \mu \mathrm{l}$ \\
\hline $10 \mathrm{mM}$ dNTPs & $3.2 \mu \mathrm{l}$ \\
\hline Sense primer $(10 \mu \mathrm{M})$ & $2 \mu \mathrm{l}$ \\
\hline Antisense primer $(10 \mu \mathrm{M})$ & $2 \mu \mathrm{l}$ \\
\hline GoTaq G2 DNA polymerase $(5 \mathrm{U} / \mu \mathrm{l})$ & $0.2 \mu \mathrm{l}$
\end{tabular}

\subsubsection{Protein expression analyses}

\subsubsection{Flow cytometry}

CMs were digested using collagenase $B$ and Trypsin-EDTA as described in 2.2.1.11. Collected cells were washed twice with DPBS ( $200 \times \mathrm{g}, 5 \mathrm{~min})$ and fixed in 4\% PFA solution for 20 min at RT. After removing of the PFA solution, cells were washed twice with DPBS ( $270 \times \mathrm{g}, 5 \mathrm{~min}$ ) and incubated with the primary antibody diluted in $0.1 \%$ Triton-X/BSA at $4^{\circ} \mathrm{C}$ overnight. After three washing steps ( $5 \mathrm{~min}, 270 \times \mathrm{g}, 0.1 \%$ Triton-X/BSA), cells were incubated with the secondary antibody in $0.1 \%$ Triton-X/BSA for $45 \mathrm{~min}$ at RT under exclusion of light. After three washing steps, samples were resuspended in $200 \mu \mathrm{l} 0.2 \%$ 
BSA-PBS and transferred into FACS tubes. For the calibration of the flow cytometer, non-labeled cells were used. Routinely, 10,000 cells were measured per sample. Gates had been adjusted to cells stained only with the secondary antibody.

\subsubsection{Western blot analyses}

\subsection{Preparation of cell lysates}

For cell lysis, frozen cell pellets were extensively resuspended in 80-100 $\mu \mathrm{l}$ cell lysis buffer and incubated for $30 \mathrm{~min}$ on ice. To get rid of bigger cell fractions, lysates were centrifuged $\left(2,400 \times \mathrm{g}, 5 \mathrm{~min}, 4^{\circ} \mathrm{C}\right)$ and the supernatant was transferred into a new reaction tube. $5 \mu$ l of each sample was diluted in $95 \mu$ l DPBS for measurements of protein concentrations. The protein concentration was determined using the Pierce BCA protein assay kit according to the manufacturer's instructions and a 96-well photometer at 562 $\mathrm{nm}$. The samples were directly prepared for SDS-PAGE or stored at $-80^{\circ} \mathrm{C}$ until further use. Before SDS-PAGE, a total amount of $40 \mu \mathrm{g}$ protein lysate was mixed with SDS loading buffer and DPBS in a volume of $20 \mu \mathrm{l}$ and denatured for $30 \mathrm{~min}$ at $37^{\circ} \mathrm{C}$.

\subsection{SDS-polyacrylamide gel electrophoresis}

By SDS-PAGE charged proteins are separated according to their molecular weight. The separation gel was mixed according to Table $\mathbf{2 1}$ and poured between two glass plates separated by spacers and fixed with clamps. Solutions for the separation gel were covered with a thin layer of isopropanol. After polymerization the isopropanol was decanted, a stacking gel was poured on top and a comb was inserted. After the polymerization of the stacking gel, the whole gel was attached to an electrophoresis chamber, filled with $1 \mathrm{x}$ running buffer and the samples were loaded together with a prestained molecular weight marker. Electrophoresis was performed at a constant current of $30 \mathrm{~mA}$ for approx. 2 hours. To visualize the separated proteins, the gels were further processed by immunoblotting (2.2.5.2.3).

Table 21: Components for $12 \mathrm{ml}$ separation gel and $7.5 \mathrm{ml}$ stacking gel

\begin{tabular}{llll}
\hline Separation gel & $\mathbf{6 \%}$ & $\mathbf{1 2 \%}$ & $\mathbf{1 5 \%}$ \\
\hline Rotiphorese gel 30 & $2 \mathrm{ml}$ & $4.8 \mathrm{ml}$ & $6 \mathrm{ml}$ \\
\hline $4 x$ Tris/SDS pH 8.8 & $3 \mathrm{ml}$ & $3 \mathrm{ml}$ & $3 \mathrm{ml}$ \\
\hline $\mathrm{ddH}_{2} \mathrm{O}$ & $6.6 \mathrm{ml}$ & $4.2 \mathrm{ml}$ & $3 \mathrm{ml}$ \\
\hline $10 \%$ APS & $48 \mu \mathrm{l}$ & $48 \mu \mathrm{l}$ & $48 \mu \mathrm{l}$ \\
\hline Temed & $18 \mu \mathrm{l}$ & $18 \mu \mathrm{l}$ & $18 \mu \mathrm{l}$ \\
\hline
\end{tabular}

\begin{tabular}{ll}
\hline Stacking gel & 2 Gels \\
\hline Rotiphorese gel 30 & $1 \mathrm{ml}$ \\
\hline 4x Tris/SDS pH 6.8 & $1.88 \mathrm{ml}$ \\
\hline $\mathrm{ddH}_{2} \mathrm{O}$ & $4.62 \mathrm{ml}$ \\
\hline $10 \%$ APS & $37.5 \mu \mathrm{l}$ \\
\hline Temed & $15 \mu \mathrm{l}$ \\
\hline
\end{tabular}




\subsection{Protein transfer and detection}

Proteins previously separated by SDS gel electrophoresis (2.2.5.2.2) were transferred to PVDF membranes using the Wet/Tank blotting system. Four filter-papers were soaked in $1 \mathrm{x}$ transfer buffer. PVDF membrane was activated according to the manufactures instructions. Two filter-papers were placed on the black site of the holder cassette and the SDS gel was placed on top followed by the PVDF membrane and two additional filter papers. Air bubbles were removed by rolling a pipette over the stack. The protein transfer was continuously cooled with ice during the blotting (400 mA, $2 \mathrm{~h}$ ).

Free binding sites on the PVDF membrane were blocked with 3\% BSA/TBS-T or nonfat dry milk according to the listed dilution in Table 8 (60 min, RT). Immunostaining was performed by incubation with primary antibodies diluted in 1-3\% BSA/TBS-T $\left(4^{\circ} \mathrm{C}\right.$, overnight), followed by HRP-conjugated secondary antibodies in 1\% BSA/TBS-T (60 min, RT). In between, the membrane was extensively washed three times with TBS-T. Antigens of interest were detected by adding ECL reagents and the result was documented with a chemiluminescence detection system.

\subsubsection{Immunofluorescence analyses}

The expression of pluripotency-associated proteins in undifferentiated hiPSCs, germ layer-specific proteins in spontaneously differentiated hiPSCs, as well as cardiac-specific proteins in CMs was shown by immunostaining. The hiPSCs, spontaneously differentiated cells and CMs were initially cultivated on coverslips. Cells were washed twice with DPBS, fixed with 4\% PFA (20 min, RT), and washed again three times. Unspecific binding sites were blocked with $1 \%$ BSA (overnight, $4^{\circ} \mathrm{C}$ ). Cells for nuclear-, cytoplasmic- and cardiac-specific protein analyses were additionally permeabilized with $0.1 \%$ Triton $X$ 100/BSA (10 min, RT). Stainings with CX43 were not treated with $0.1 \%$ Triton X. For the staining the primary antibody was added (overnight, $4^{\circ} \mathrm{C}$ ) followed by the secondary antibody (60 min, RT, dark). The nuclei were stained with DAPI (1:5000 diluted in $\mathrm{ddd}_{2} \mathrm{O}$, $10 \mathrm{~min}, \mathrm{RT}$, dark). Between all steps the samples were washed three times with DPBS. Prior to mounting, coverslips were washed with $\mathrm{dd}_{2} \mathrm{O}$ and mounted on microscope slides using Fluoromount-G. HiPSCs and spontaneously differentiated cells were analyzed by Carl Zeiss Axio Observer.Z1 microscope. Immunostaining of CMs was visualized by confocal laser microscopy using a 40x 1.1.W objective using the oil immersion technique. 


\subsubsection{Generation of cell clones edited by CRISPR/Cas9}

\subsubsection{Transfection of HEK293T cells using calcium-phosphate}

For the cleavage assay of CRISPR/Cas9, calcium-phosphate transfection was performed in HEK293T cells which are susceptible for transfection by calcium-phosphate precipitates. The DNA is enclosed in the calcium-phosphate precipitates and is taken up by the cells across the cell membrane. For the transient transfection $3 \times 10^{5}$ cells were seeded in each well of a 6-well plate one day before transfection. 4 hours before transfection, medium was exchanged with fresh FL-medium. $3.3 \mu \mathrm{g}$ plasmid-DNA was added to $16.6 \mu \mathrm{l} 2.5 \mathrm{M}$ $\mathrm{CaCl}_{2}$ and adjusted with $\mathrm{ddH}_{2} \mathrm{O}$ to a final volume of $166 \mu \mathrm{l}$. Afterwards $166 \mu \mathrm{l} 2 \mathrm{x} \mathrm{HBS}-$ solution was added dropwise with additional snapping of the reaction tube. Immediately afterwards, the mixture was added to the cells. 24 hours post transfection, medium was removed and $300 \mu \mathrm{l}$ of pre-warmed $10 \%$ glycerol $(\mathrm{v} / \mathrm{v})$ was added to the cells and removed shortly afterwards. Cells were washed once with fresh medium. Next, transfected cells were counted and pellets containing $1 \times 10^{6}$ transfected cells were prepared by pooling 2-3 transfected wells as described in 2.2.1.13 and used for the CRISPR/Cas9 cleavage assay.

\subsubsection{Cleavage assay of CRISPR/Cas9}

When using CRISPR/Cas9 editing tools to obtain targeted genome correction, it is necessary to determine how efficient the Cas9 nuclease cleaves the target sequence. The cleavage assay of CRISPR/Cas9 was done using the GeneArt genomic cleavage detection Kit according to the manufacturer's instructions. Briefly, pellets containing $1 \times 10^{6}$ CRISPR/Cas9 transfected HEK293T cells were resuspended in $50 \mu$ l cell lysis buffer/protein degrader mix, heated in a thermal cycler and used as template for the PCR, which amplified the DNA region of interest. For the cleavage assay, $2 \mu \mathrm{l}$ of the PCR product was mixed with detection reaction buffer in a total volume of $10 \mu \mathrm{l}$. The re-annealing reaction was started in the thermal cycler. Immediately afterwards, the PCR-amplicon containing the mismatched DNA was cleaved by the detection enzyme and the entire sample was load on a $2 \%$ agarose gel using Ficoll loading buffer without dye to avoid interference with band intensity measurements. The percent of gene modification was analyzed using Alpha Imager Software. 


\subsubsection{Transfection of hiPSCs}

Transfection of hiPSCs was performed using the Nucleofector II device and the nucleofector kit $1 / 2$ for human stem cells. For this purpose, feeder-free hiPSCs were passaged 2 days before transfection. For one transfection, $4 \mu \mathrm{g}$ plasmid and $3.3 \mu \mathrm{g}$ ssODNs were added to the mixture of $82 \mu \mathrm{l}$ Nucleofector solution and $18 \mu \mathrm{l}$ supplement 1 . HiPSCs with confluence about $80-90 \%$ were detached as described in 2.2.1.8. For each transfection $2 \times 10^{6}$ cells were transferred into a $15 \mathrm{ml}$ falcon tube and centrifuged ( $200 \times g, 5 \mathrm{~min})$. The supernatant was discarded and the cells were carefully resuspended in the DNA-Nucleofector solution mix and transferred into the Nucleofector cuvette. The cuvette was placed in the Nucleofector II device and program B-16 was used. Transfected cells were transferred into one well of a 6-well plate containing E8 medium supplemented with TZV and $1 \times \mathrm{P} / \mathrm{S}$.

\subsubsection{FACS of GFP ${ }^{+}$hiPSCs}

To select successfully transfected cells, GFP ${ }^{+}$hiPSCs were purified in the FACS facility of the UMG Göttingen 24 hours after transfection. To this end, hiPSCs cells were washed twice with Versene, incubated for $5 \mathrm{~min}$ and resuspended in FACS medium. Cell suspension was filtered with $50 \mu \mathrm{m}$ filcons and transferred to FACS tubes. $1-7 \times 10^{3}$ cells were directly seeded on each Geltrex-coated 96-well plate in a 1:1 mix of E8 and MEFconditioned E8 medium supplemented with additional hbFGF (10 ng/ml), 1x TZV and 1x $\mathrm{P} / \mathrm{S}$. E8 medium supplemented with hbFGF (10 ng/ml), 1x TZV 1x P/S was changed every third day.

\subsubsection{Expansion of cell clones edited by CRISPR/Cas9}

First colonies were observed 10-18 days after FACS. Single hiPSC colonies were shortly incubated with Versene (30 sec). After aspirating Versene, hiPSC colonies were resuspended with E8 medium plus $1 \times$ TZV using a $100 \mu \mathrm{l}$ pipette and transferred into Geltrex-coated 96-well plates or 48-well plates depending on the colony size. Confluent 48-well plates were transferred onto two wells of a 24-well plate as described in 2.2.1.8. Confluent wells were used either for pellets for DNA extraction or for cryopreservation. For the cryopreservation of CRISPR/Cas9 clones, cells were washed once with Versene and incubated for approx. two minutes with Versene. After aspirating Versene, hiPSCs were directly resuspended in CRISPR/Cas9 freezing medium and transferred into cryo tubes. Cells were kept overnight at $-80^{\circ} \mathrm{C}$ in a freezing box. 


\subsubsection{Genomic DNA isolation and screening of cell clones edited by CRISPR/Cas9}

For screening of cell clones edited by CRISPR/Cas9, cell pellets were resuspended in $80 \mu \mathrm{l}$ QuickExtract solution and incubated in a thermoshaker $\left(65^{\circ} \mathrm{C}, 800 \mathrm{rpm}\right)$. In the next step, the temperature was increased to $98^{\circ} \mathrm{C}$ (2 minutes, $800 \mathrm{rpm}$ ) and DNA lysates were centrifuged $\left(1 \mathrm{~min}, 16,000 \times \mathrm{g}\right.$ ). DNA lysates were stored at $-20^{\circ} \mathrm{C}$ or directly used for PCR amplification of CRISPR/Cas9 targeted sequences. PCR was performed as described in Table 20 using a reaction of $50 \mu \mathrm{l}$ containing $5 \mu$ l DNA lysate and the appropriate primer set. The primer set RYR2C-1021 or CPVTC-Seq was used for amplification of transfected cells from patient $\mathrm{c} 1$ and $\mathrm{c2}$, and the primer set CPVTb-Seq was used for amplification of cells transfected from patient b1. $1 \mu$ restriction enzyme was directly put into $25 \mu \mathrm{l}$ PCR product and incubated for 3 hours in a thermoshaker $\left(37^{\circ} \mathrm{C}, 300 \mathrm{rpm}\right)$. After restriction digestion, the PCR product was separated on an 1.5\% agarose gel for $60-70$ minutes at 120 V. Positive digested clones were visualized using ultraviolet light. The undigested PCR products of these clones were purified using the QIAquick gel extraction kit according to the manufacturer's instructions. Purified DNA was sent out for sequencing.

\subsubsection{Subcloning of sequenced CRISPR/Cas9 edited cell clones}

When sequencing showed inconsistent results, CRISPR/Cas9-edited cell clones were digested as described in 2.2.6.4 and resuspended in FACS medium to dilute those again using the FACS facility. To increase the survival rate after sorting, just living cells were gated and different numbers of cells (ranging from 1-11 cells) were put into one well of a Geltrex-coated 96-well plate containing 1:1 mixture of MEF-conditioned E8 medium and E8 medium supplemented with additional hbFGF (10 ng/ml), 1x TZV and 1x P/S.

\subsubsection{Cytosolic calcium recordings}

5 to 7 days before the calcium recording, CRISPR/Cas9-edited CMs, patient-specific CMs, and Ctrl-CMs were digested and plated on Geltrex-coated $25 \mathrm{~mm}$ glass coverslips and incubated in B27 medium. For calcium imaging cells (day 86-118) were washed twice with Tyrode's solution, incubated with $5 \mu \mathrm{M}$ fluo-4/AM fluorescent calcium indicator and $0.02 \%\left[\mathrm{w} / \mathrm{v}\right.$ ] pluronic F-127 in Tyrode's solution for 30 minutes at $20-22^{\circ} \mathrm{C}$ and washed twice again with Tyrode's solution. Recordings were obtained from paced cells which were field stimulated at $0.25 \mathrm{~Hz}(18 \mathrm{~V}, 3 \mathrm{~ms}$ duration) using a recording chamber with platinum electrodes in Tyrode's solution at $20-22^{\circ} \mathrm{C}$ and total medium change every ten minutes. Cells were treated with isoprenaline $(100 \mathrm{nM})$ for 10 minutes before recordings. Images were captured using a LSM 710 confocal microscopy system and a 63x 1.4 NA oil 
objective in line scan mode (512 pixels, $45 \mu \mathrm{m}, 1057.7 \mathrm{~Hz}, 20,000$ cycles) exiting at $488 \mathrm{~nm}$ and collecting emission at $490-540 \mathrm{~nm}$. Post-acquisition analysis of changes in intracellular calcium as a function of the time was performed using ImageJ and Excel. A defined plot per recording (500 ms, $25 \mu \mathrm{m}$ ) during diastolic phase was analyzed by ImageJ SparkMaster (settings: 1057.70 lps; $0.088 \mu \mathrm{m}$; Fl. U. 0; criteria 3.3; intervals 3) and sparks with minimal amplitude of $0.2 \Delta \mathrm{F} / \mathrm{F} 0$, minimal width of $0.7 \mu \mathrm{m}$ and minimal duration of $7 \mathrm{~ms}$ were selected for detailed analysis. 


\section{Results}

\subsection{Clinical profile of patients suffering from CPVT}

In this study, CPVT patients were recruited to study mutation-specific differences as well as the molecular mechanisms of CPVT in these individuals. The study group consisted of 6 individuals (mean age 36, range 29-45 years, 5 females) who were molecularly defined heterozygous carrier of different RYR2 mutations causing CPVT symptoms. CPVT patients carried the following missense mutations: R420W (patients a2 and a3), A2254V (patient b1), E4076K (patients $\mathrm{c} 1$ and $\mathrm{c} 2$ ) and H4742Y (patient d1). Mutation nomenclature was based on RYR2 reference protein sequence NM_001035.2. The mutations are located in the four hot spot clusters of RYR2. The mutations R420W, A2254V and E4076K are localized in the cytosolic $\mathrm{N}$-terminal or central regions of the receptor and were already described in the literature (Bauce et al., 2002; Postma et al., 2005) while the mutation $\mathrm{H} 4742 \mathrm{Y}$ is located in the pore region close to the C-terminal part of the RYR2 receptor (Fig. 7). All mutations were associated with severe exercise-induced ventricular tachycardia (VT). 5 of 6 patients were known to have a family history with sudden cardiac death. Following diagnosis of CPVT, $\beta$-adrenergic blocking agents (bisoprolol or metoprolol) were administrated as first line therapy to prevent further arrhythmias. After several years, all patients had recurrent symptoms despite treatment with $\beta$-adrenergic blocking. For this reason, therapies were supplemented with the anti-arrhythmic agent flecainide to reduce the severity of arrhythmias. However, combined treatment therapies were not fully protective to prevent symptoms for longer periods. Last examinations (06/2016-07/2016) revealed still recurrent symptoms in all patients. Patient a2 is a 35 -year-old woman, who suffered since the age of 14 from palpitations during exercise and emotions. She developed premature ventricular contractions (PVCs) during exercise test without medications. Combined application of flecainide and bisoprolol showed bigeminy as most severe type of arrhythmia during exercise test. The 34-year-old female patient a3 suffered from palpitations during exercise for several years. Exercise tests without medication revealed non-sustained polymorphic VT. In 2016, renal denervation was performed due to persistent arrhythmia despite therapy with $\beta$-blockers and flecainide. The 45-year-old male patient b1 was asymptomatic before diagnosis, but screened due to familiar accumulation of sudden cardiac death. His worst arrhythmias during exercise test were couplets, which were still observed during treatment with bisoprolol and flecainide. To minimize these symptoms, left cardiac sympathetic denervation was performed in 2016. 


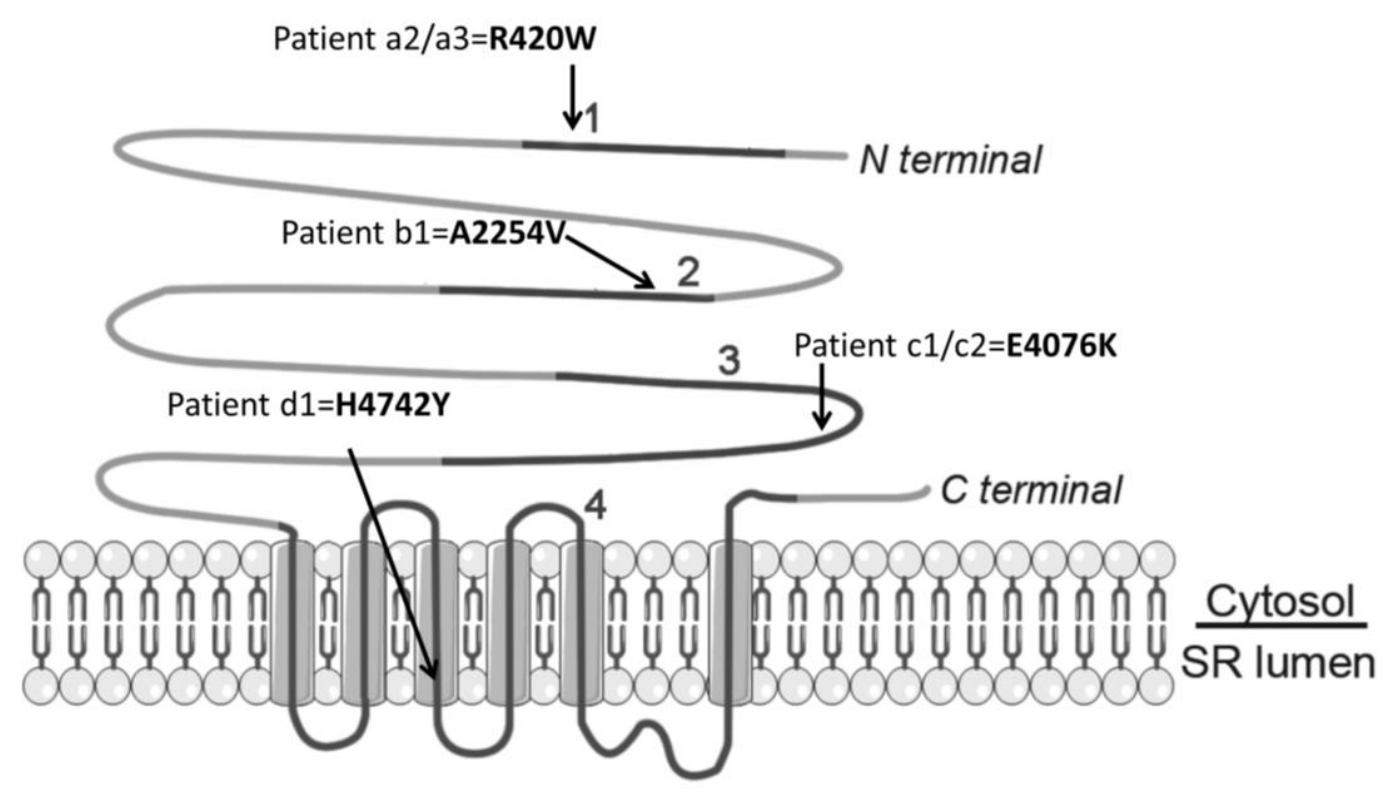

Figure 7. RYR2 protein and mutations studied in the present study. Six patients had heterozygous missense mutations in this study (arrows) which are located in different mutation clusters of the RYR2 protein. Clusters are represented as dark grey lines numbered from 1 to 4 . Cluster 1 comprises of amino acids (aa) 44-466, cluster 2 aa 2246-2534 and cluster 3 aa 37784201. These three clusters are located in the N-terminal and central regions of RYR2 and form the cytoplasmic domain. Cluster 4 comprises of aa 4497-4959 and is located in the transmembrane and $\mathrm{C}$-terminal region. Five patients $(\mathrm{a} 2, \mathrm{a} 3, \mathrm{~b} 1, \mathrm{c1}, \mathrm{c2})$ had mutations in the $\mathrm{N}$-terminal and central region of the protein and are located in the cytoplasmic domain. One Patient d1 (H4742Y) had a missense mutation in the transmembrane region which is located in the C-terminal region in the membrane. In the 3D-tetramer structure of RYR2 (not shown), the mutation H4742Y is located in the pore region of the tetrameric protein RYR2 (modified from Penttinen et al. 2015).

The 28-year-old female patient $\mathrm{c} 1$ had her first clinical symptoms already with 10 years. Left cardiac sympathetic denervation was performed due to syncope despite treatment with metoprolol. In 2010, flecainide was administrated because of recurrent ventricular arrhythmias. The 45-year-old female patient c2 had syncope at rest with the age of 27 years. Due to family history of sudden cardiac death, exercise test was performed and showed polymorphic non-sustained VT. Despite combined treatment with metoprolol and flecainide, bigeminy was observed during exercise test but the severity of arrhythmias was decreased. The 29-year-old female patient d1 had recurrent syncope with the age of 16 in response to physical and emotional stress. Exercise test showed polymorphic nonsustained VT. Ventricular arrhythmias occurred despite $\beta$-blocker therapy and additional treatment with flecainide could also not prevent bigeminy. 


\subsection{Generation of CPVT- hiPSCs and proof of their pluripotency}

\subsubsection{Generation of CPVT-hiPSCs from 3 different CPVT patients}

As part of this work, skin biopsies from three CPVT patients (b1, c1, c2) were obtained for the generation of hiPSCs. These patients had a missense mutation at different loci (A2254V, E4076K). Outgrowing skin fibroblasts were expanded. Between passage 2 and 4 fibroblasts were directly taken for transduction using the non-integrative SeV containing the Yamanaka factors KFL4, C-MYC, OCT4 and SOX2 (Fusaki et al., 2009b) (Fig. 8). Four weeks after transduction, several colonies resembling pluripotent stem cells with respect to their round morphology were picked mechanically and transferred onto new culture dishes coated with MEFs (Fig. 9A-C). From each patient between two and four independent cell clones were further cultivated and characterized for their pluripotency and differentiation ability.



Figure 8. Timeline for the generation of CPVT-hiPSCs. Generation of pluripotent stem cells involves cultivation of skin fibroblasts obtained from skin biopsies and reprogramming using Sendai virus. After successful reprograming, hiPSC colonies were expanded and characterized for their pluripotency and differentiation ability into all three germ layers.
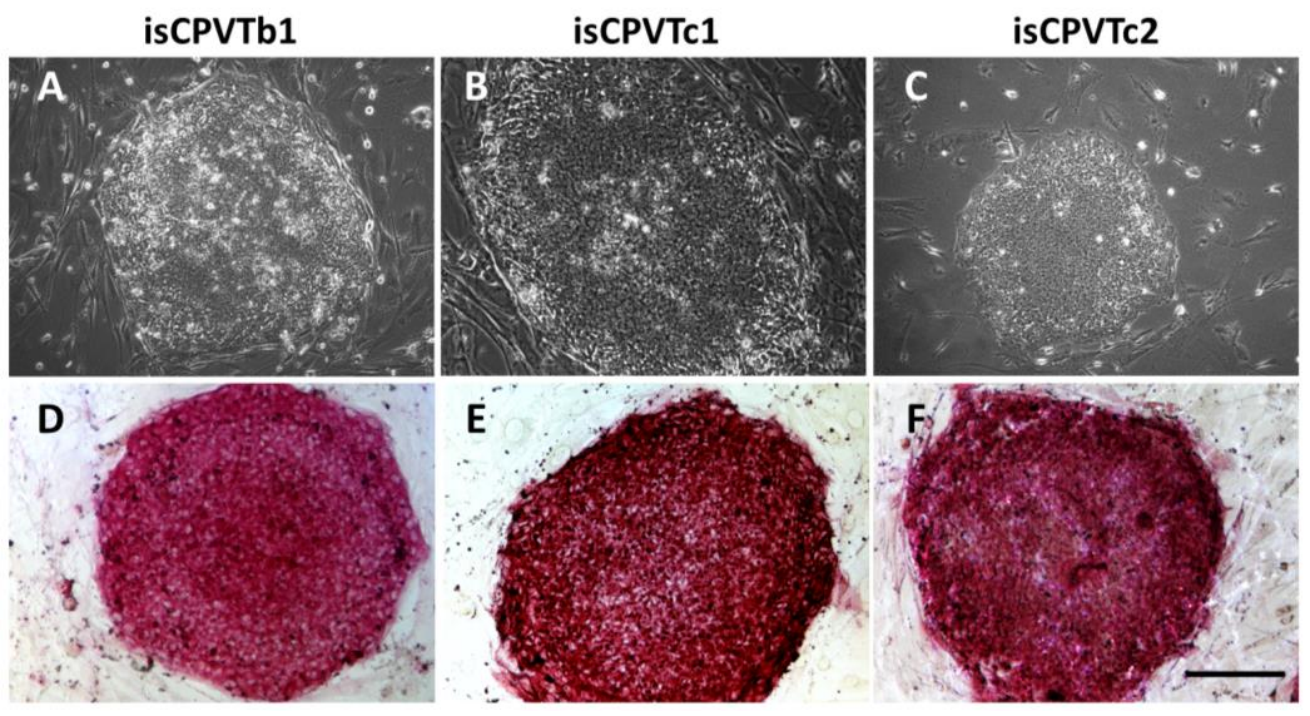

Figure 9. Morphology and alkaline phosphatase activity of CPVT-hiPSCs. CPVT-hiPSC lines were first cultured on MEFs. Like human pluripotent stem cells, all tested cell lines have typical round shape colonies (A-C) and express high levels of alkaline phosphatase (D-F). Scale bar: $200 \mu \mathrm{m}$. 
The enzyme alkaline phosphatase, which is the first marker for undifferentiated cells (O'Connor et al., 2008), was expressed in all generated CPVT-hiPSCs indicated by red staining of CPVT-hiPSC colonies (Fig. 9D-F). Cellular reprogramming involves an extensive remodeling of gene expression. Expression of pluripotency-specific markers was proven by reverse transcriptase-PCR (Fig. 10) on mRNA level and immunofluorescence staining (Fig. 11) on protein level.

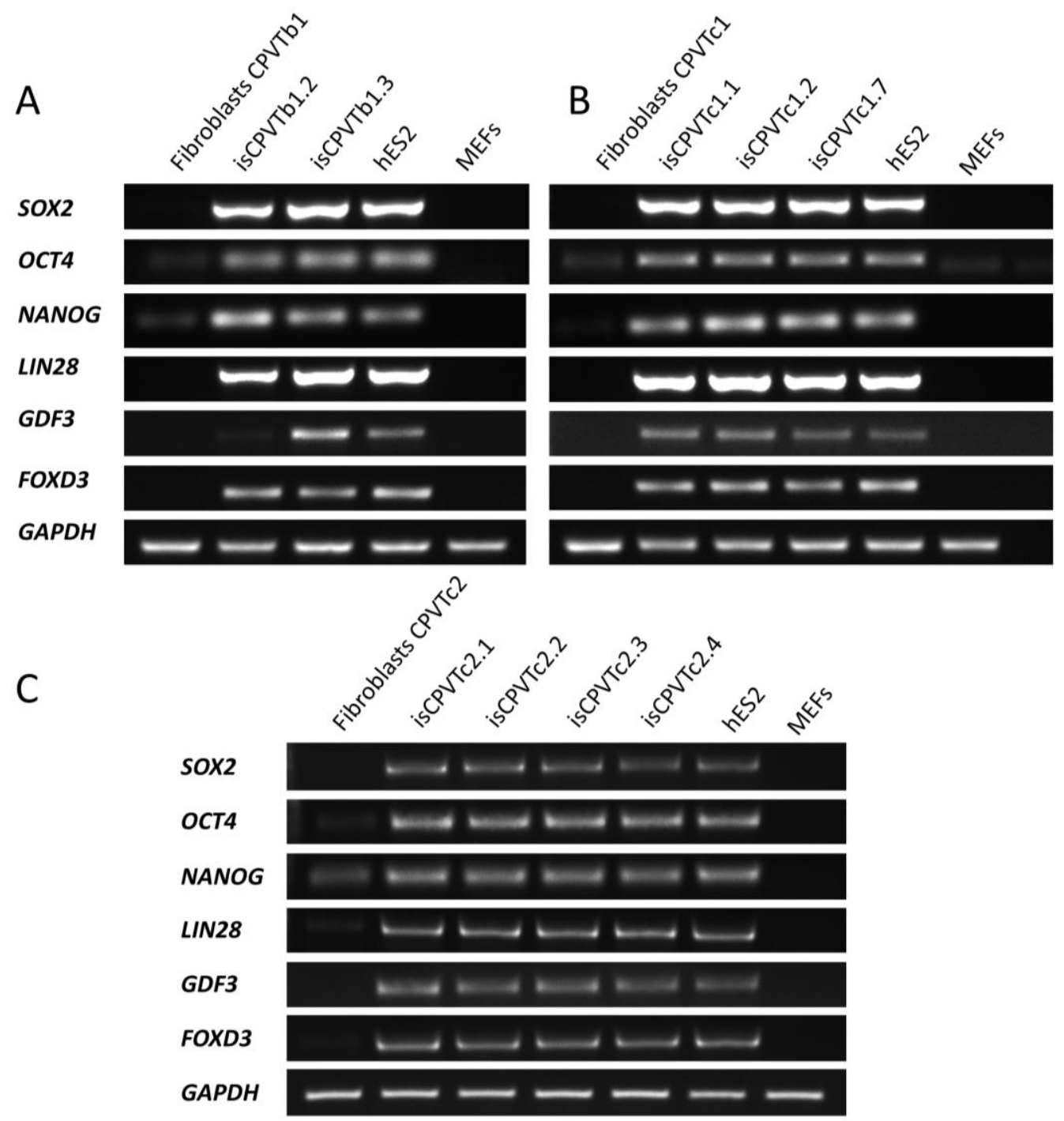

Figure 10. Gene expression analysis of generated CPVT-hiPSCs and their parental fibroblasts. All CPVT-hiPSC lines from patients b1 (A), c1 (B) and c2 (C) show similar high expression of the pluripotency-related genes SOX2, OCT4, NANOG, LIN28, GDF3, and FOXD3 compared to the hES2 cell line. These pluripotency genes were expressed at very low levels in their parental fibroblasts. GAPDH was used as housekeeping gene. MEFs were used as negative control. The hESC line hES2 was used as positive control.

In comparison to hESCs (hES2), CPVT-hiPSCs from patients b1, c1 and c2 expressed similar levels of the pluripotency-related marker genes OCT4, NANOG, SOX2, LIN28, GDF3 and 
FOXD3 (Fig. 10). In contrast, pluripotency markers are not expressed in their parental fibroblasts. Just a very weak expression of NANOG and OCT4 was detected in fibroblasts. These data support an activation of endogenous gene expression because four of analyzed genes (NANOG, LIN28, GDF3, and FOXD3) are not part of SeV which was used for reprogramming.


Figure 11. Immunostaining detects pluripotency-related proteins of generated CPVT-hiPSCs. Representative cell lines from patient $\mathrm{c} 1$ are shown here, demonstrating high expression of the transcription factors SOX2 (A-C), NANOG (D-F) and OCT4 in the nuclei (G-I), as well as for the cytoplasmic located marker LIN28 (J-L). SSEA4 (M-O) and TRA-1-60 (P-R), both located in the cell membrane, were also detected. The cell nuclei were stained with DAPI. Scale bar: $100 \mu \mathrm{m}$. 
Furthermore, the pluripotency related proteins NANOG, OCT4, SOX2, LIN28, SSEA4, and TRA-1-60 were detected in all generated hiPSC clones (Fig. 11). The transcription factors SOX2, NANOG and OCT4 (Fig. 11A-I) are located in the nuclei of all CPVT-hiPSC lines. LIN28 is located in the cytoplasm (Fig. 11J-L), whereas SSEA4 (Fig. 11M-O), and TRA1-60 (Fig. 11P-R) are located on the membrane surface. No obvious differences were observed among all analyzed hiPSC clones.

\subsubsection{Differentiation potential of in vitro and in vivo}

Differentiation ability of generated hiPSCs was determined in vitro by spontaneous differentiation via EB formation and in vivo by teratoma formation. For in vitro differentiation, CPVT-hiPSCs were cultivated in suspension with Iscove medium for 8 days. During this period, cells formed multi-cellular aggregates known as EBs, which were plated onto gelatin-coated culture dishes at day 8 after differentiation. To analyze changes of gene expression, mRNA was isolated and reverse transcribed into CDNA at three different time points (day 0 , day $8+3 /$ d $8+17$, and day $8+25$ ) (Fig. 12).

In all differentiation experiments, CPVT-hiPSC lines showed an increased mRNA expression of tissue-specific germ layer markers in a developmentally controlled manner. All cell lines from patients b1 and $c 1$ showed an upregulation of the tested germ layer markers. However, not all cell lines from patient c2 express all tested germ layer markers after differentiation on mRNA level (Fig. 12C). In general, the early endodermal marker gene AFP was already expressed at day 8 , whereas $A L B$, a late marker for hepatocytes, was often expressed at later stages during the process of differentiation. The mesodermal marker genes $C T N T$ and $\alpha-M H C$ were already early expressed during the differentiation experiments. Both ectodermal marker genes, SYN and TH were expressed at early stages, too.

To test spontaneous differentiation on protein level, differentiated hiPSCs were stained with antibodies against three different germ layer-specific proteins (Fig. 13). HiPSC lines from all three CPVT patients were able to express the endodermal marker AFP (Fig. 13AG), the mesodermal marker $\alpha$-SMA (Fig. $\mathbf{1 3 H}-\mathbf{N}$ ) as well as the neuroectodermal marker $\beta$-III-TUBULIN (Fig. 130-U) on protein level after spontaneous differentiation. 

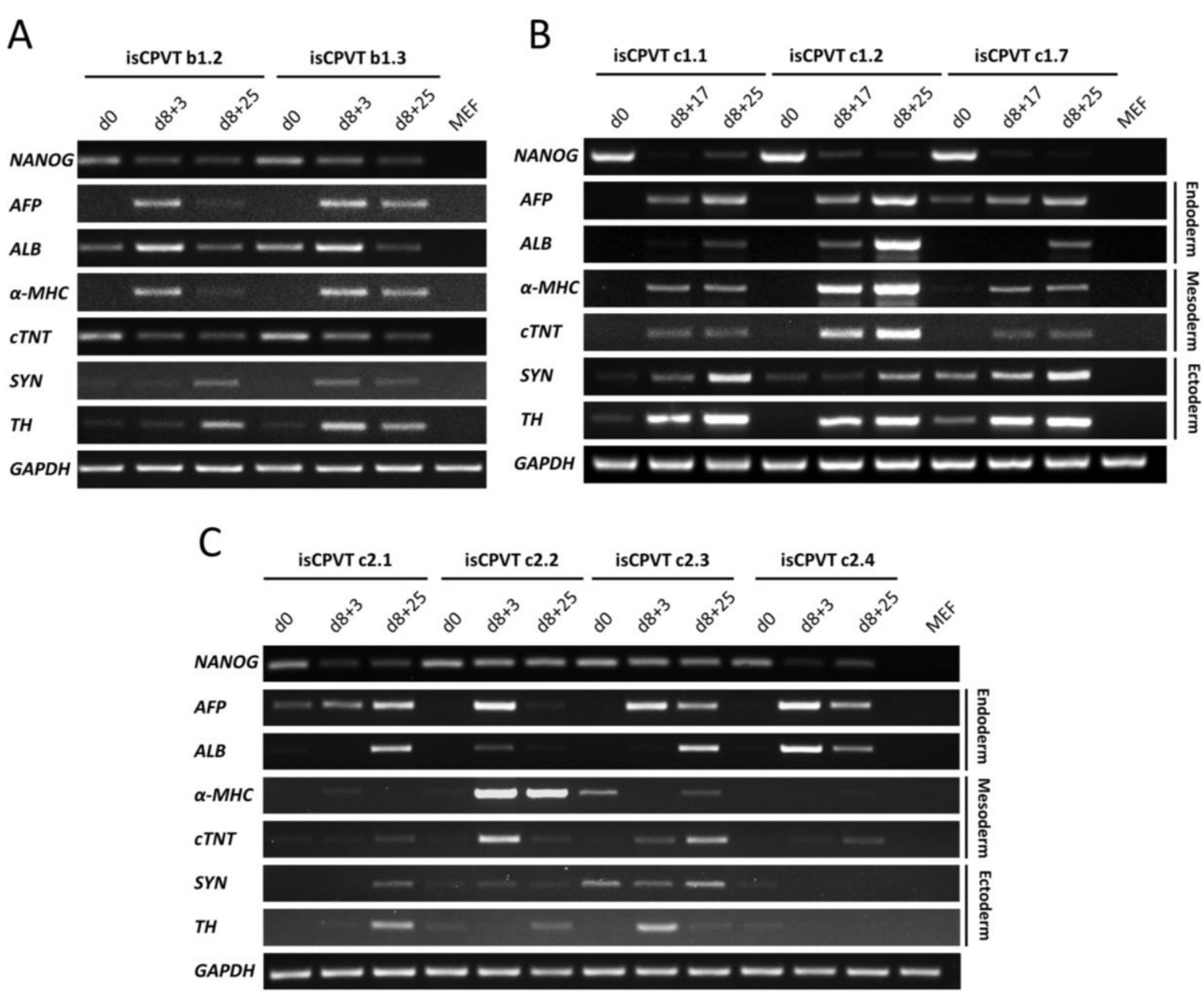

Figure 12. Gene expression of differentiated CPVT-hiPSCs. CPVT-hiPSCs from patients b1 (A), c1 (B), and c2 (C) were differentiated spontaneously in vitro and mRNA was isolated at day 0 , day $8+3 / \mathrm{d} 8+17$, and day $8+25$ of differentiation. For each germ layer two representative markers were chosen. Gene expression of endodermal (AFP, ALB), mesodermal ( $\alpha-M H C, C T N T)$ and ectodermal markers $(S Y P, T H)$ was upregulated during spontaneous differentiation. The pluripotency marker NANOG was highly expressed in undifferentiated hiPSCs and downregulated during differentiation. GAPDH was used as housekeeping gene. MEFs were used as negative control.

To test the differentiation potential in vivo, hiPSCs were injected subcutaneously into immunodeficient Rag2 $2^{-/} \mathrm{yc}^{-/-}$mice to induce teratoma formation. The injected cells formed mature teratoma, which were analyzed histologically for the expression of all three germ layers (Fig. 14). Teratomas derived from patients b1 and $c 1$ contained derivatives of all three embryonic germ layers shown by neural rosettes representing ectoderm (Fig. 14AC), cartilage representing mesoderm (Fig. 14D-H) and intestinal tissue representing endodermal lineages (Fig. 14I-M). Neural rosette structures were not found in the teratoma derived from the cell line isCPVTc1.1. HiPSC lines from patient $\mathrm{c} 2$ developed just a single teratoma which expressed mesodermal tissues such as cartilage and endodermal tissue such as intestinal tissue (Fig. 14H, M) but lacked expression of neural rosettes. 


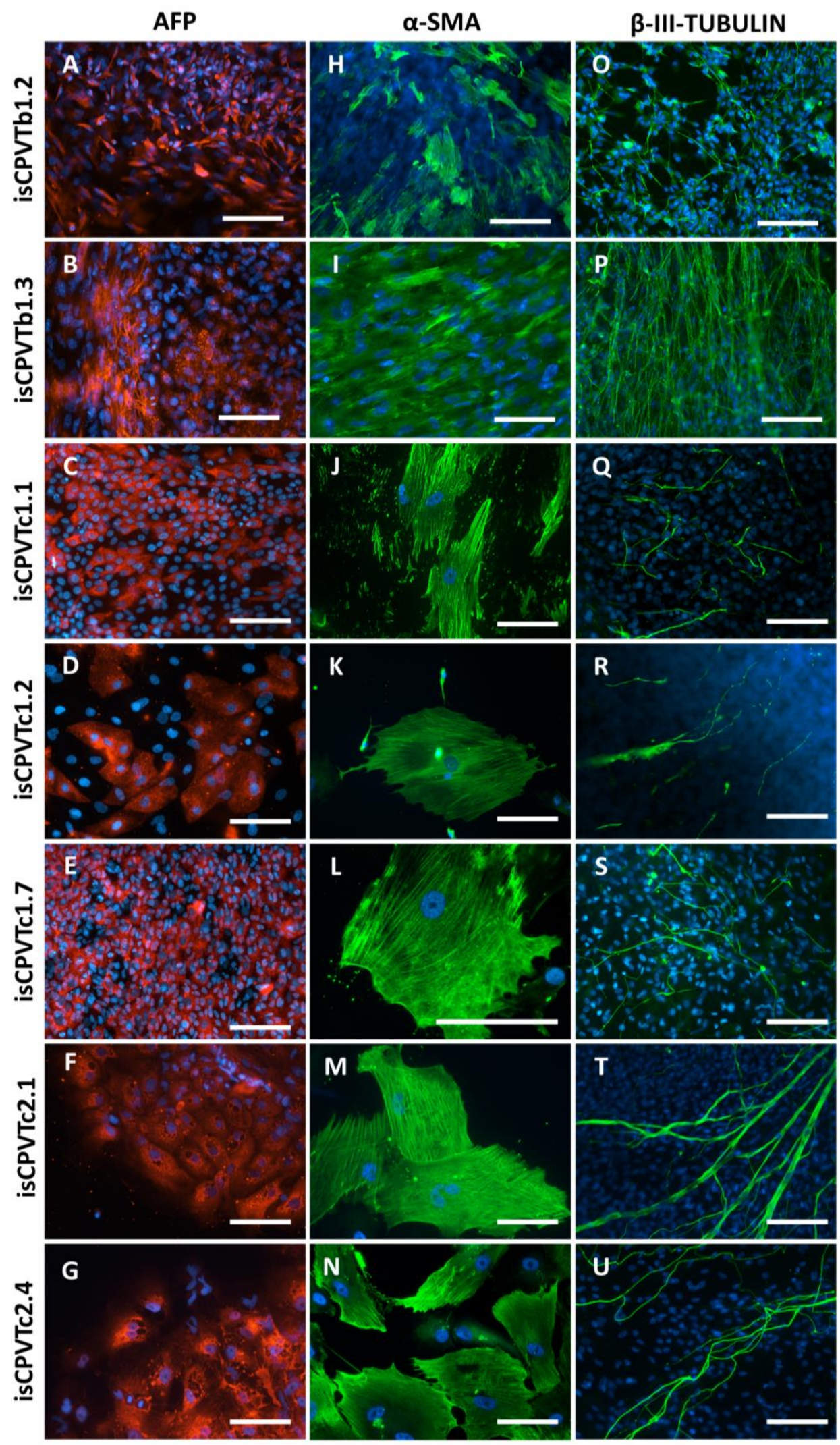

Figure 13. Immunostaining of differentiated hiPSCs detects germ layer-specific proteins. All differentiated CPVT-hiPSC lines were positive for the endodermal marker AFP (A-G), mesodermal marker protein SMA (H-N) and the ectodermal marker $\beta$-III-TUBULIN (O-U). The cell nuclei were stained with DAPI. Scale bar: $100 \mu \mathrm{m}$. 


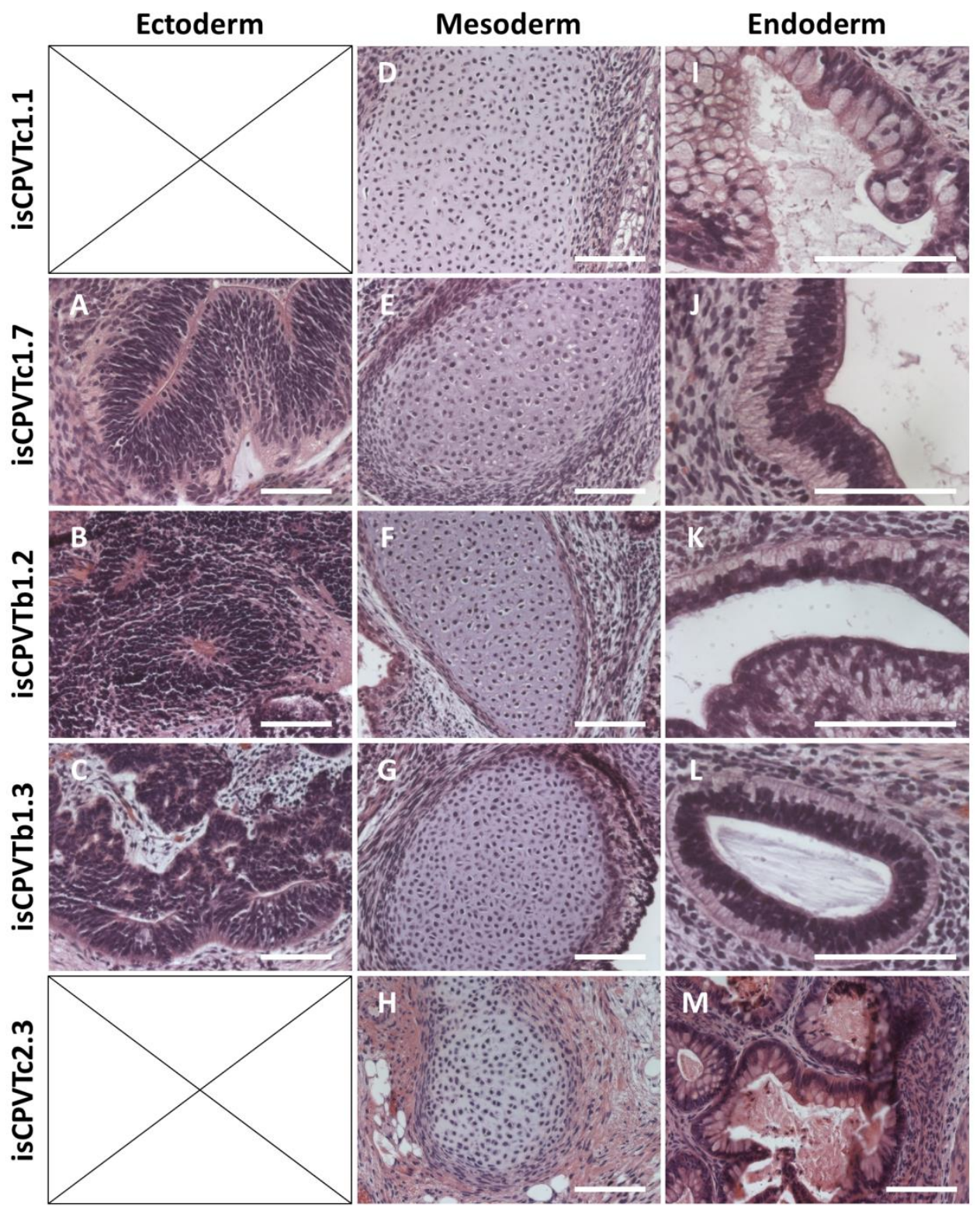

Figure 14. Teratoma formation of CPVT-hiPSCs. After subcutaneous injection into Rag $2^{-1} \gamma c^{-1-}$ mice, nearly all injected CPVT-hiPSC lines developed teratoma (A-M) with derivatives of all three embryonic germ layers: ectoderm represented by neural rosettes $(\mathbf{A}-\mathbf{C})$, mesoderm represented by cartilage (D-H), and intestinal tissue with endodermal origin (I-M). Neural rosettes were not observed in the hiPSC lines isCPVTc1.1 and isCPVTc2.3. Scale bar: $200 \mu \mathrm{m}$.

\subsubsection{Verification of the RYR2 missense mutation in CPVT-hiPSCs}

RYR2 gene regions, containing different mutation sites (A2254V, E4076K) in three CPVT patients (b1, c1, c2), were sequenced to confirm the genotype of CPVT-hiPSCs. RYR2 is located on chromosome 1q43. The genomic DNA of generated CPVT-hiPSCs from patients b1, c1 and c2 was isolated and sequenced approximately 170-250 bp up- and downstream of the predicted mutations within the RYR2 gene. CPVT-hiPSC lines from patient b1 showed the missense mutation A2254V referred to the amino acid coding 
sequence. The amino acid position E4076K is mutated in CPVT-hiPSC lines from patients c1 and c2 (Fig. 15).
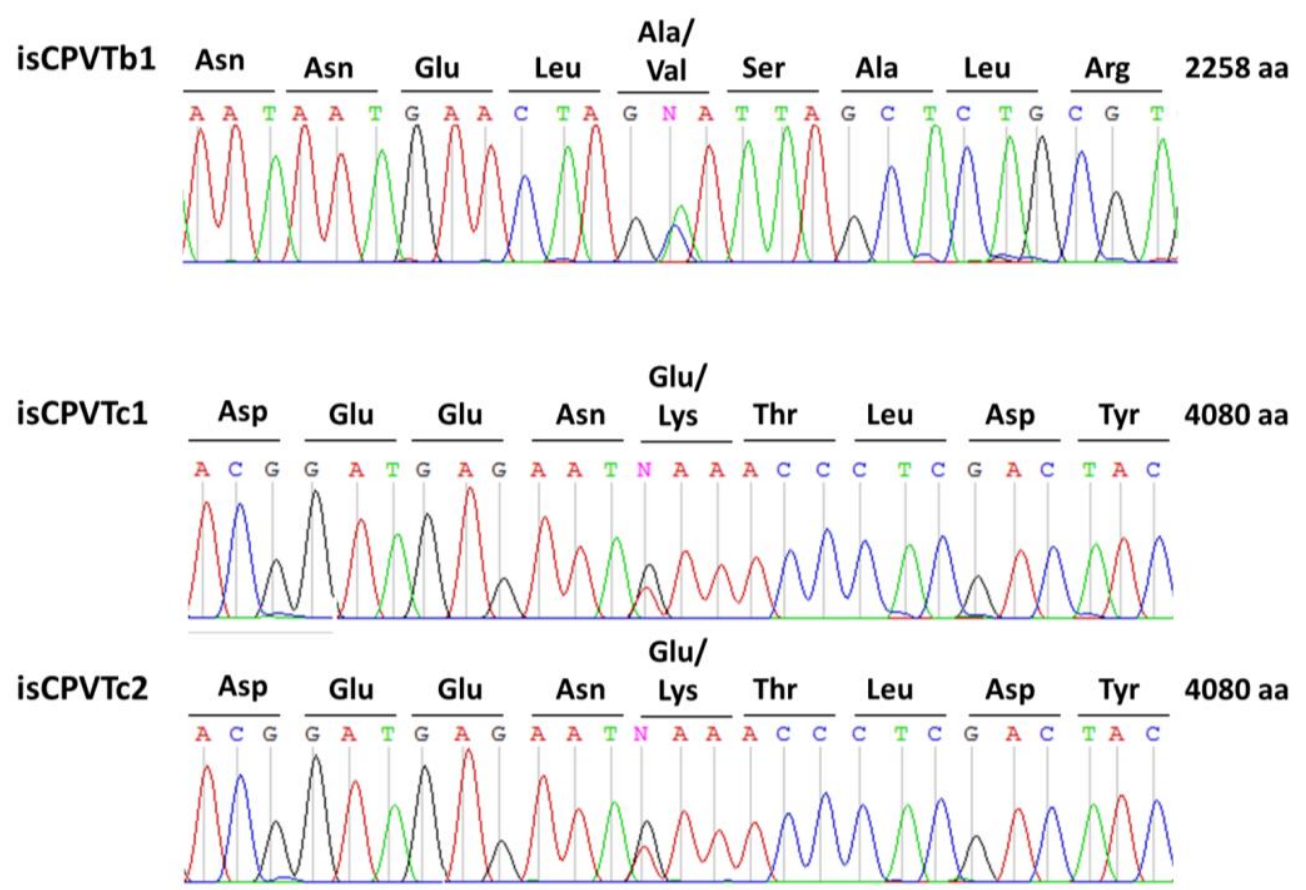

Figure 15. Verification of the RYR2 point mutation in the generated CPVT-hiPSCs. Sequence chromatograms from CPVT-hiPSCs revealed the expected heterogeneous missense mutation in the RYR2 gene (A2254V, E4076K). The analysis of hiPSCs from patient b1 shows a nucleotide substitution of $C$ to $T$ in exon 44 in $R Y R 2$ converting alanine to valine at position 2254 of the protein. HiPSCs from patients $\mathrm{c} 1$ and $\mathrm{c} 2$ had nucleotide substitution of $\mathrm{G}$ to $\mathrm{A}$ in exon 90 which changes the amino acid from glutamic acid into lysine at codon 4076.

To sum up, patient-specific CPVT-hiPSCs were successfully generated using nonintegrative reprogramming methods. CPVT-hiPSCs fulfill all criteria which are essential for pluripotent stem cells. All generated hiPSC lines from 3 CPVT patients had a typical morphology of pluripotent stem cells and expressed pluripotency-associated markers on mRNA and protein level. Furthermore, in vitro experiments showed that CPVT-hiPSCs were able to differentiate spontaneously into all three germ layers indicated by mRNA and protein expression of germ layer-specific markers. Teratoma assays demonstrated pluripotency of the analyzed CPVT-hiPSC lines in vivo. Sequencing confirmed their missense mutation in $R Y R 2$ which probably induces the CPVT phenotype in patients.

\subsection{Directed differentiation of hiPSCs into CMs}

After reprogramming and cultivation on MEF, Ctrl-hiPSCs and CPVT-hiPSCs from patients a2, a3, b1, c1, c2 and d1 were adapted to Geltrex-coated plates and maintained in feederfree culture conditions using E8 medium. Direct CM differentiation was initiated with 
cardio differentiation medium and progressive treatment with $\mathrm{CHIR}$ and IWP2 for temporal WNT signaling activation and repression, respectively (Fig. 16).
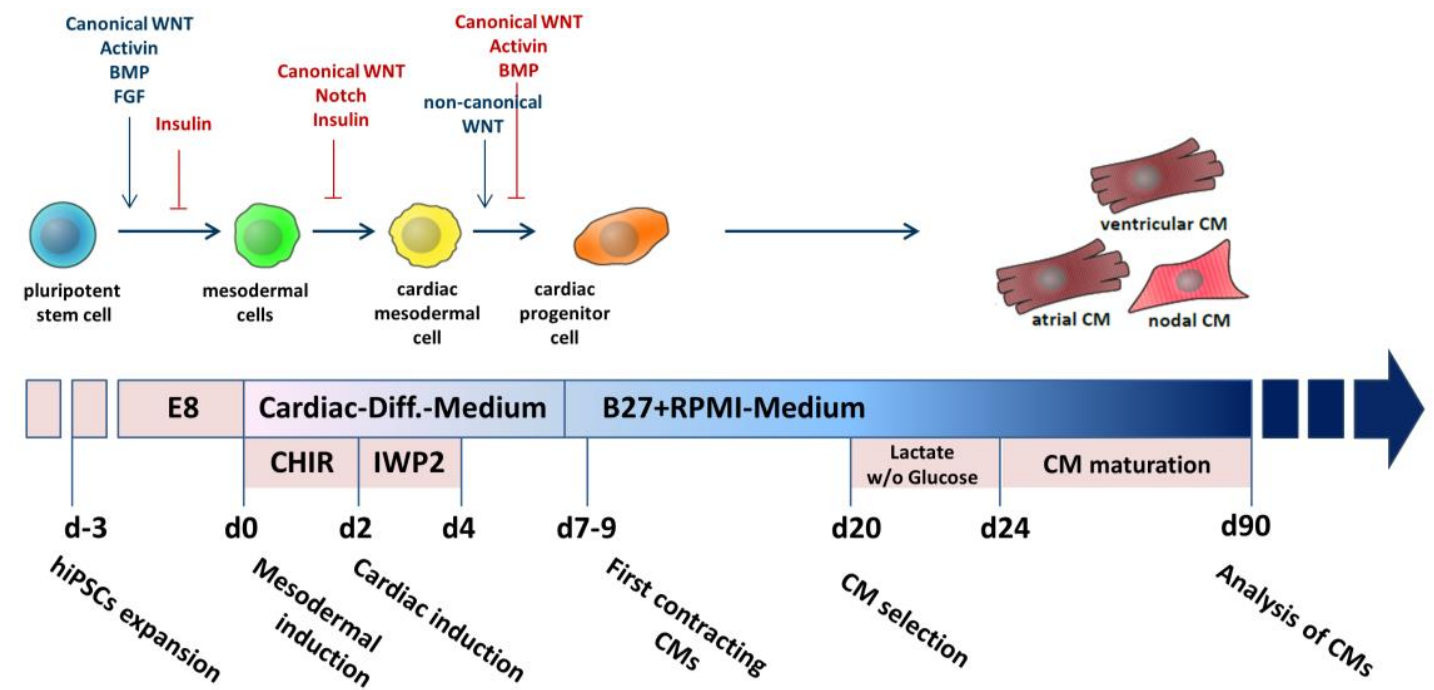

Figure 16. Schematic illustration of the directed cardiac differentiation using hiPSCs. At $80-90 \%$ confluence of hiPSC cultured on Geltrex-coated plates, direct $C M$ differentiation was initiated using GSK3 inhibitor CHIR to activate canonical WNT/ $\beta$-catenin signaling for mesodermal induction. For cardiac specification, the canonical WNT pathway was inhibited by IWP2 for two days, thereby cardiac gene expression was activated. CMs were selected using medium containing lactate instead of glucose and cultured for three months until further analysis (Modified from Cyganek et al. 2013).

First beating areas were observed between day 8 and 12 after starting differentiation. To purify young differentiated CMs (<30 days), metabolic CM selection was performed using RPMI1640 minus glucose, but supplemented with lactate for 4-6 days. For maturation, CMs were cultured in RPMI1640 with B27 for 90-115 days before further analysis. Flow cytometry analysis reveals high efficiency of cardiac differentiation and high purity of CMs after lactate selection and cultivation for three months. Up to $98 \%$ of the CMs were stained positive for cTNT (Fig. 17).

A

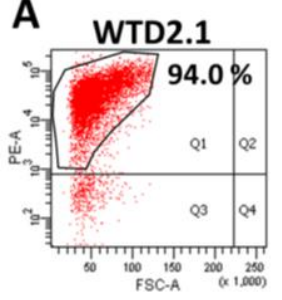

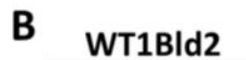

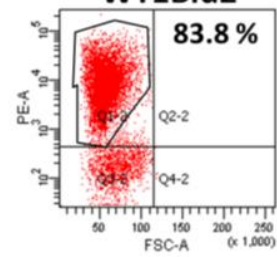

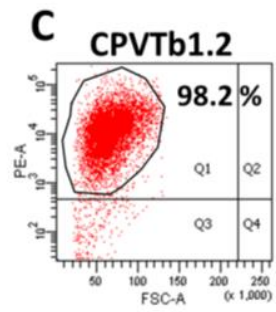

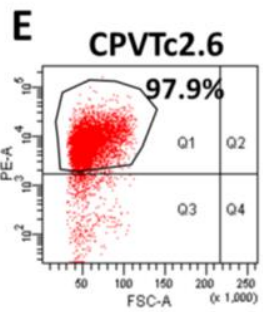

Figure 17. Flow cytometric analysis of 3-month-old CMs. Ctrl-CMs (A, B) and CPVT-CMs (C, D, E) were stained with antibodies against cTNT and detected with PE-labeled anti-mouse IgG antibodies. In total, we obtained an average of $92.3 \%$ CTNT $^{+}$cells ( $n=14$ different experiments from different cell lines). The diagrams show $\mathrm{CTNT}^{+}$cells of representative measurements of Ctrland CPVT-CMs that were gated for living cell populations. Unstained CMs served as negative control. 10,000 cells were measured per sample. 
Reverse transcription PCR analyses show that Ctrl- as well as CPVT-CMs expressed cardiac-specific markers such as CTNT, MLC2A and MLC2V, $\alpha-A C T, I R X 4, R Y R 2$ and CASQ2 on mRNA level (Fig. 18). Although depending on the cell quality, individual batch-to-batch differences were observed for the expression of CASQ2, $\alpha-A C T$ and the ventricular marker IRX4, no significant differences were observed between Ctrl- and CPVT-CMs analyzed.

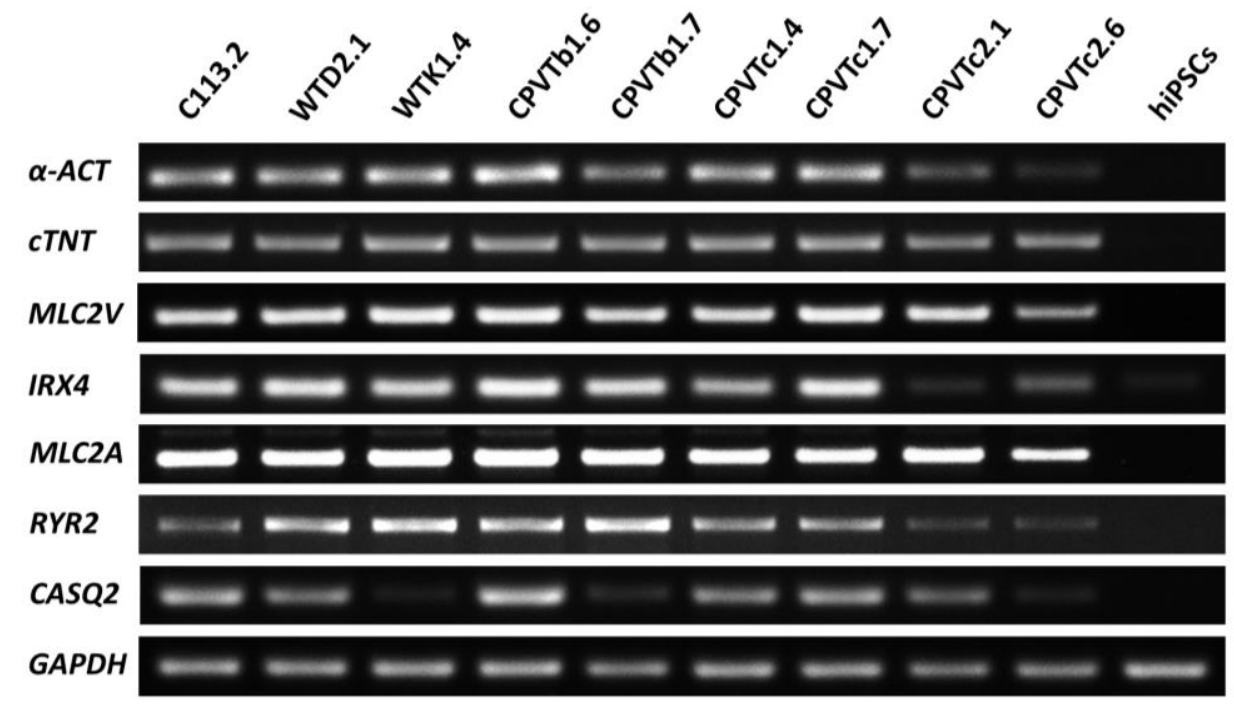

Figure 18. Expression of cardiac-specific marker genes. Different CPVT- and Ctrl-hiPSCs were directly differentiated into $\mathrm{CMs}$. After three months of cultivation, mRNA was isolated and reverse transcribed into cDNA. CPVT- and Ctrl-CMs expressed cardiac specific genes such as cTNT, $\alpha-A C T$, $M L C 2 V, M L C 2 A$ and RYR2 on mRNA level.

Immunofluorescence staining of differentiated CPVT-CMs showed normal organized cross-striations indicated by sacromeric staining with antibodies against RYR2, $\alpha-A C T$, MLC2A and MLC2V (Fig. 19). RYR2 expression co-localized with the expression of $\alpha$-ACT. Furthermore, the gap junction protein $\mathrm{CX} 43$, which is important for conducting electrical signals between neighboring cells, was detected between adjacent CMs (Fig. 20). No specific differences were observed between Ctrl- and CPVT-CMs across all cell clones regarding cardiac-specific structural proteins. Most $\mathrm{CMs}$ show a ventricular-like phenotype indicated by predominant expression of the ventricular-specific marker MLC2V (Fig. 19), which is restrictedly expressed in the ventricles throughout development and persists into adulthood (Chuva de Sousa Lopes et al., 2006). CMs positive for MLC2A were also detected (Fig. 19), which is expressed in both atria and ventricles during development (Chuva de Sousa Lopes et al., 2006). Furthermore, patch-clamp analysis displayed that most cells had action potentials with ventricular-like shapes (data from W. Li). 


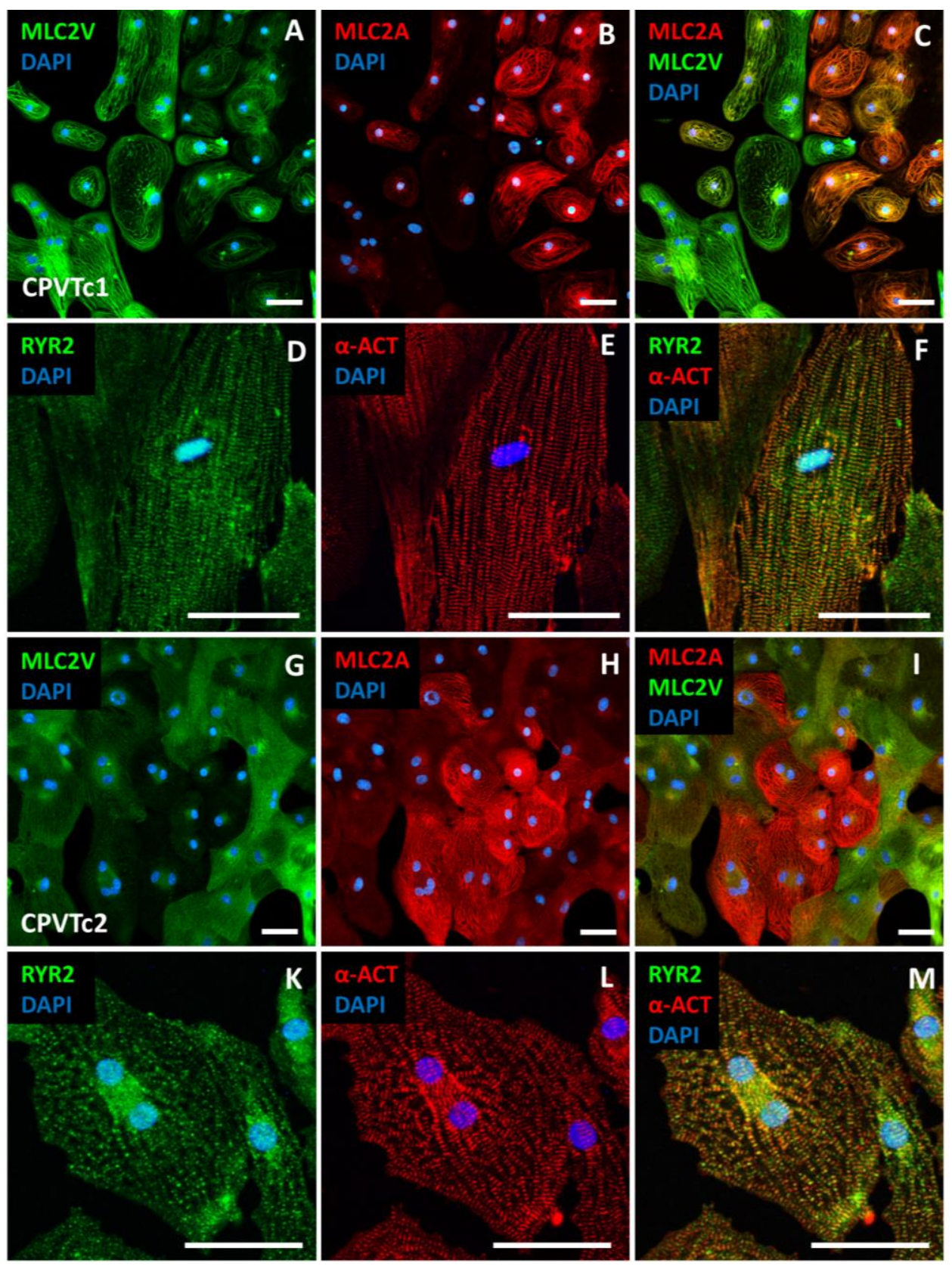

Figure 19. Cardiac differentiation of CPVT-hiPSCs. 3-months-old CMs from patients $\mathrm{C1}$ (A-F) and c2 (G-M) expressed the structural myofilament proteins $\alpha-A C T(E, L), M L C 2 A(B, H)$ and MLC2V $(A, G)$. The striated pattern of RYR2 expression (D, K) is co-localized with expression of $\alpha-A C T$ (F, $M)$. Cell nuclei were stained with DAPI (blue). Same results were obtained from CMs from patient b1 (not shown). Scale bar: $50 \mu \mathrm{m}$. 


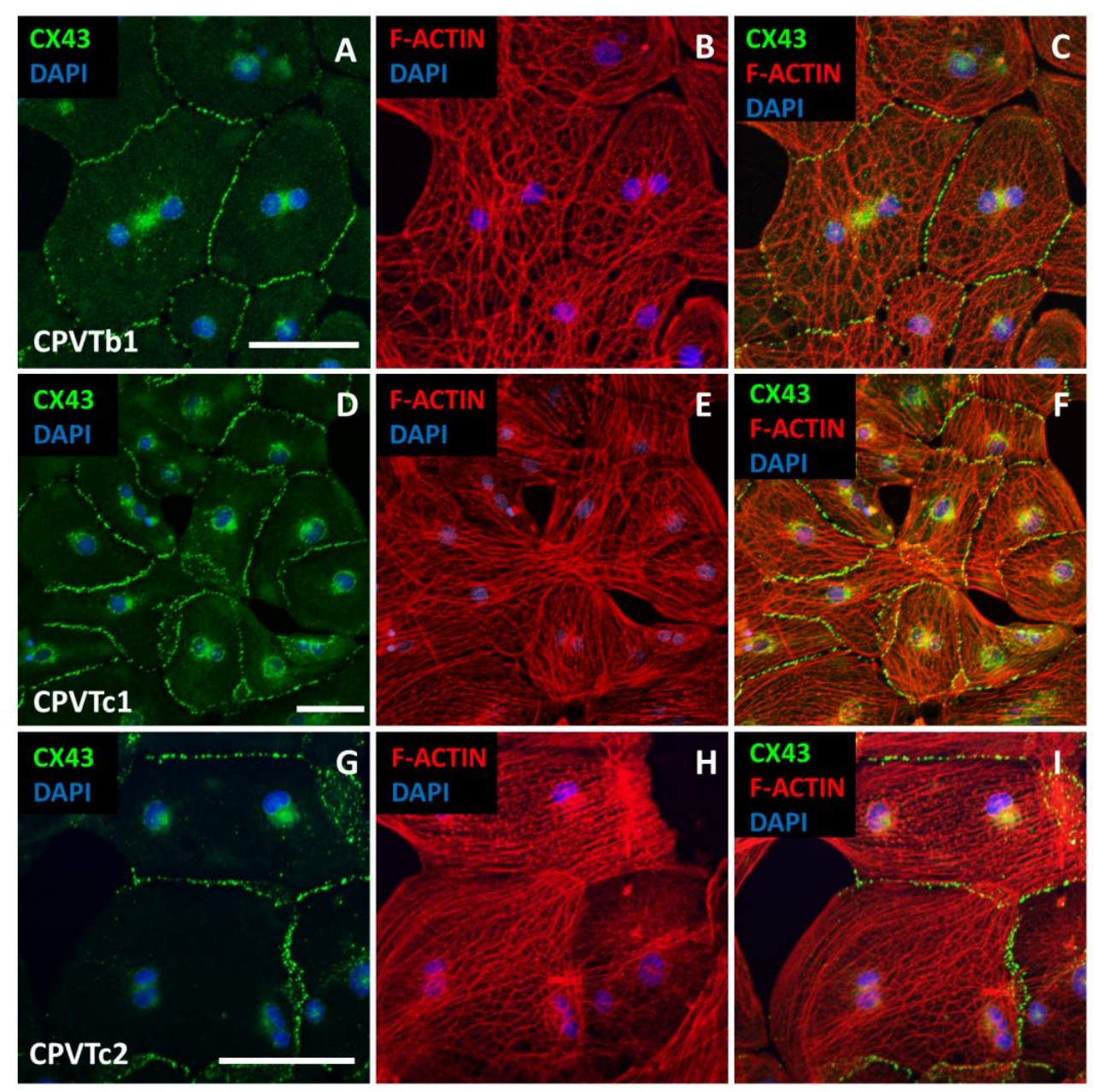

Figure 20. Expression of CX43 in CPVT-CMs. The gap junction protein CX43 was detected at the connection between adjacent CMs of patients b1, c2 and c2 (A, D, G, in green). CPVT-CMs were stained with Alexa 555-conjucated phalloidin to visualize the cytoskeleton of cellular F-ACTIN filaments (B, E, H, in red). Cell nuclei were stained with DAPI (blue). Scale bar: $50 \mu \mathrm{m}$.

Taken together, CMs with high purity were successfully differentiated from Ctrl- and patient-specific CPVT-hiPSCs. CMs differentiated from Ctrl-and patient-specific CPVThiPSCs express cardiac-specific markers on mRNA and protein levels and do not show patient- or cell line-specific differences regarding structural abnormalities or cardiac-specific marker expression. Furthermore, most Ctrl- and CPVT-hiPSC lines generated from STEMCCA and Sendai virus do not show differences regarding differentiation efficiencies. However, CPVT-hiPSCs from patient c2, which were generated using SeV, were difficult to differentiate into CMs. For this reason, CPVT-CMs from patient c2 were mainly differentiated with STEMCCA-derived hiPSC lines. 


\subsection{Role of heterogeneous $R Y R 2$ missense mutations in the pathogenesis of CPVT}

Electrophysiological and calcium imaging studies demonstrated, that CPVT-CMs derived from hiPSCs carrying different RYR2 mutations in the domains $a, b, c$, and $d$, could recapitulate the phenotype of the CPVT disease in vitro. CPVT-CMs show mutationspecific or patient-specific differences regarding electrophysiological properties. CPVTCMs carrying the RYR2 mutations A2254V (CPVTb) and E4076K (CPVTc) reveal phenotypic stress-dependent arrhythmia with DADs (data from W. Li) (Fig. 21) and increased spontaneous $\mathrm{Ca}^{2+}$ releases under basal and isoprenaline stimulated conditions (data from L. Cyganek) (Fig. 22). In contrast, CPVT-CMs carrying the RYR2 mutation H4742Y (CPVTd) display phenotypic stress-dependent arrhythmia with EADs (Fig. 21) and reduced spontaneous $\mathrm{Ca}^{2+}$ releases (Fig. 22). CPVT-CMs carrying the RYR2 mutation R420W (CPVTa) display phenotypic stress-dependent arrhythmia with DADs (Fig. 21) but reduced spontaneous $\mathrm{Ca}^{2+}$ releases (Fig. 22).

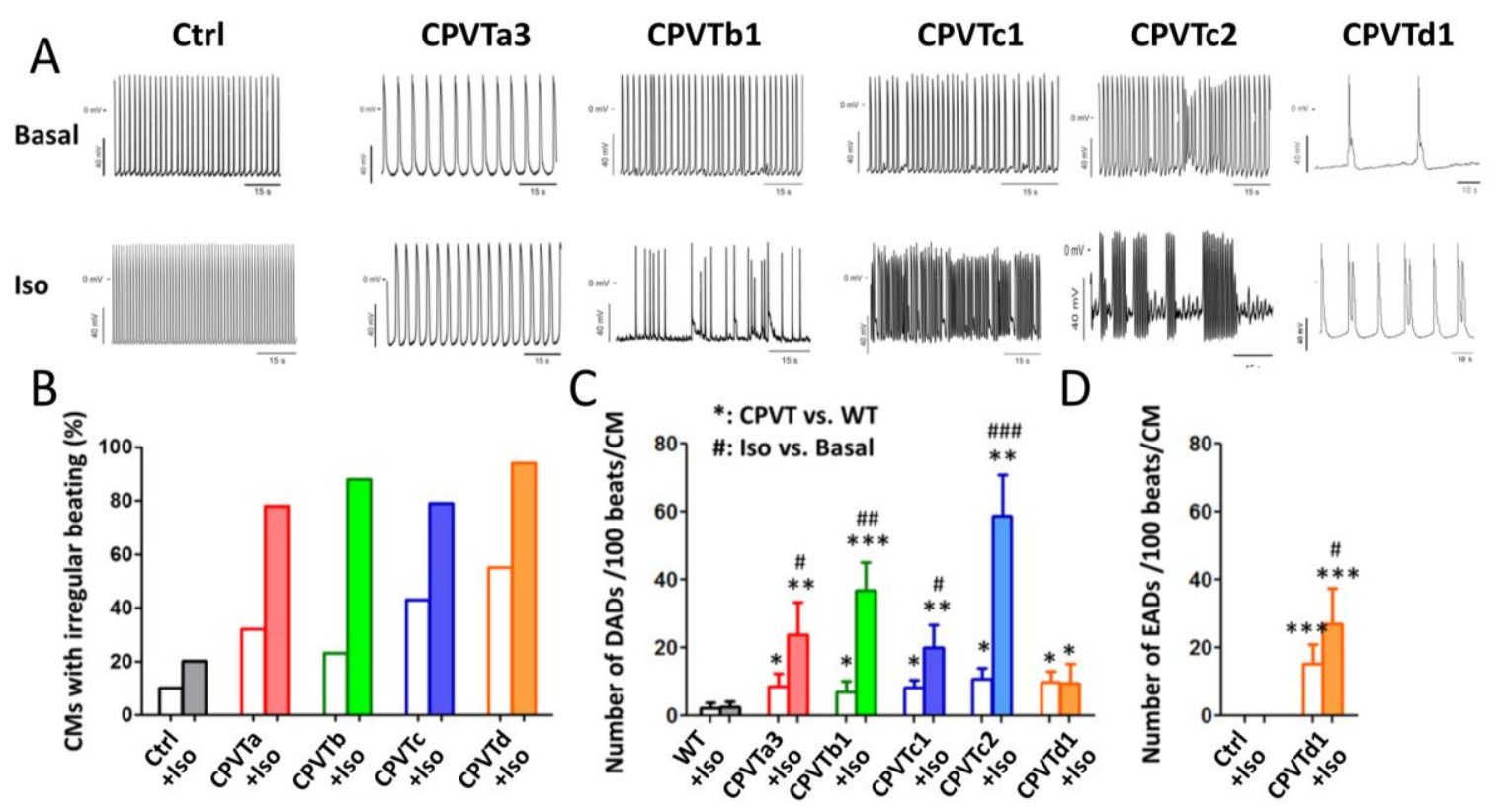

Figure 21. Patch clamp analysis of CPVT- and Ctrl-CMs. Under basal and isoprenaline-stimulated conditions (100 nM Iso) CPVT-CMs showed increased number of arrhythmic events (A, C, D) and increased frequencies of irregular beating in comparison to Ctrl-CMs (B). CPVT-CMs from patients a3 and $\mathrm{c} 1$ show a milder phenotype with increased numbers of DADs under basal conditions and isoprenaline stimulation. In contrast, CPVT-CMs from patients b1 and c2 show more severe phenotypes after isoprenaline treatment with high incidence of DADs (C). CPVT-CMs generated from patient d1 reveal phenotypic stress-dependent arrhythmia with EADs (D) (data from W. Li). 
A

A Ctrl Basal
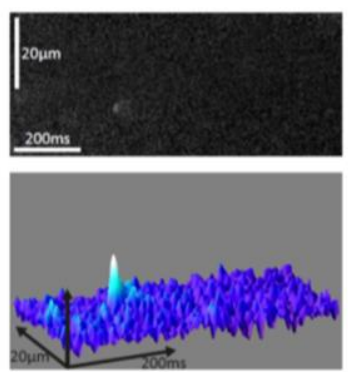

C



CPVTc2 Basal
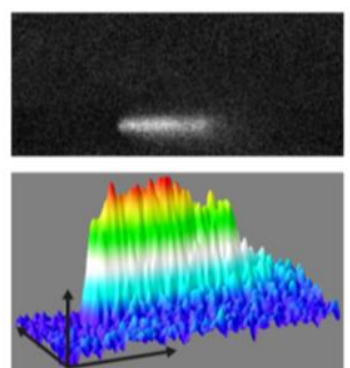

D
B Ctrl Iso


CPVTc2 Iso
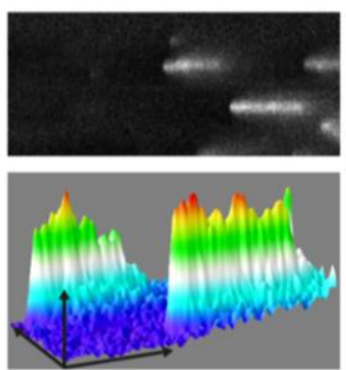

E
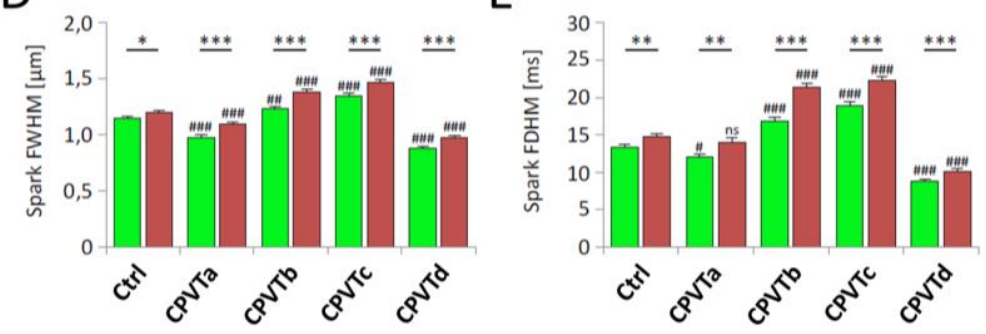

Figure 22. Basal and isoprenaline-induced $\mathrm{Ca}^{2+}$ sparks in CPVT- and Ctrl-CMs. For cytosolic calcium recordings by confocal line scan imaging of Ctrl- and CPVT-CMs, cells were loaded with fluo-4/AM, paced with $0.25 \mathrm{~Hz}$ and stimulated with Iso (100 nM). Example recordings CPVTc2-CMs show increased $\mathrm{Ca}^{2+}$ sparks visualized by 3D reconstruction of selected areas before and after stimulation with Iso (A). CPVT-CMs from patients b1, $\mathrm{c} 1$ and $\mathrm{c} 2$ display larger $\mathrm{Ca}^{2+}$ sparks with significantly higher spark amplitudes (C), an increased full width at half spark maximum (FWHM) (D) and a longer full duration at half spark maximum (FDHM) (E) compared to Ctrl-CMs. In contrast, CPVT-CMs from patients a2, a3 and d1 display smaller $\mathrm{Ca}^{2+}$ sparks with significantly lower spark amplitudes (C), a reduced FWHM (D) and a shorter FDHM (E) compared to Ctrl-CMs. However, Iso treatment increases $\mathrm{Ca}^{2+}$ sparks in all CPVT-CMs (C, D) (data from L. Cyganek).

\subsubsection{Allele-specific expression of $R Y R 2$ in CPVT-CMs on mRNA level}

To analyze the reason for the different severe phenotypes observed by patch-clamp and calcium imaging, all six different CPVT patients (a2, a3, b1, c1, c2 and d1) were analyzed for potential differences in allele-specific expression of $R Y R 2$ gene caused by the heterogeneous missense mutations (Fig. 7). To study whether an allelic imbalance of RYR2 expression exists in hiPSC-derived CPVT-CMs, RNA sequencing of the healthy and mutated allele was performed with the MiSeq sequencing system (TAL Göttingen). Total mRNA of 3-month-old CMs was isolated, reverse transcribed into CDNA and the region of interest, which included the mutation, was amplified and subsequently sequenced. The results showed that CMs derived from CPVT patients have a similar RYR2 MRNA expression of the disease causing and the healthy allele located in chromosome 1q43 (Fig. 23A, B). Only CMs from patient a3 have a slightly increased expression of the disease causing allele. In addition, gDNA from hiPSCs-derived CMs from patient c1 was sequenced 
as control and revealed similar copy numbers of the healthy allele $(51.2 \%)$ and mutated allele (47.8\%).

A

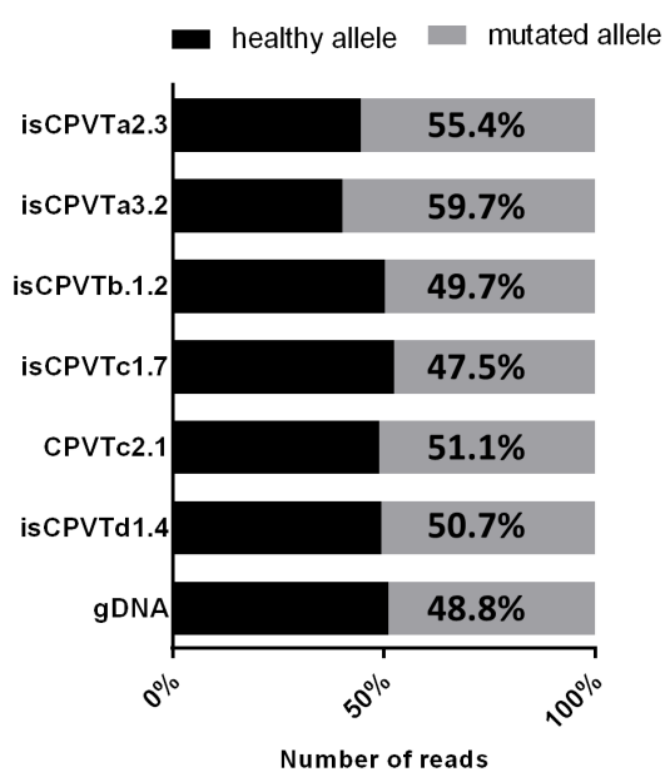

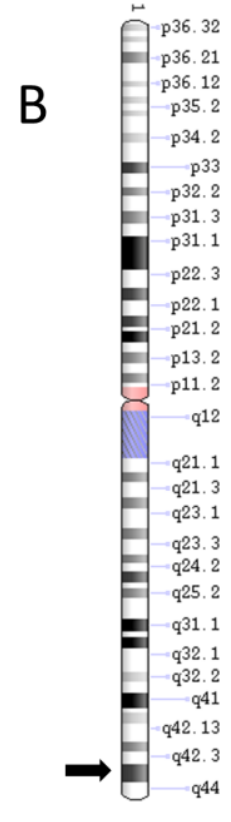

Figure 23. Allele-specific RYR2 expression of CPVT-CMs. Sequencing of cDNA copies revealed same expression of the healthy compared to the mutated allele. Just one cell line (isCPVTa3.2) showed a slight increased expression of the mutated allele (59.7\%). For each probe between $470-$ 1100 counts were made after subtraction of double cDNA copies generated by PCR amplification (A). Missense mutations are located in RYR2 of chromosome 1q43 (B).

\subsubsection{RYR2 expression and phosphorylation in CPVT-CMs}

To address the question whether different CPVT phenotypes result from different levels of RYR2 expression in CPVT-CMs, the protein amount of RYR2 from three-month-old CPVT-CMs (cell lines: isCPVTa2.3, isCPVTa3.2, isCPVTb1.2, isCPVTc1.7, CPVTc2.6, isCPVTd1.4) was compared to Ctrl-CMs (cell lines: WTD2.1, BM76.1, isWT1bld2). But western blot analysis of CPVT-CMs from all 6 patients do not show differences of RYR2 protein expression compared to Ctrl-CMs derived from healthy subjects (Fig. 24).

Different mechanisms were proposed how exactly point mutations in $R Y R 2$ induce arrhythmias and different severe phenotypes. In the next step, we tested the hypothesis that RYR2 from CPVT patients is hyperphosphorylated in response to $\beta$-adrenergic stimulation, thereby causing hyperactive channels and $\mathrm{Ca}^{2+}$ leak. To address this question, phosphorylation of Ser2808 and Ser2814, both located in the cytosolic region of RYR2, was tested in CMs derived from patients a2, a3, b1, c1 and c2 which have their mutation in the cytosolic region. To activate the adrenergic signaling pathway, three-month-old CMs were treated with isoprenaline (15 min, $100 \mathrm{nM})$, calyculin $\mathrm{A}(50 \mathrm{nM})$, or with both 
substances together. Calyculin A is a structurally distinct inhibitor of PP1 and PP2A which regulate dephosphorylation of the receptor.

A
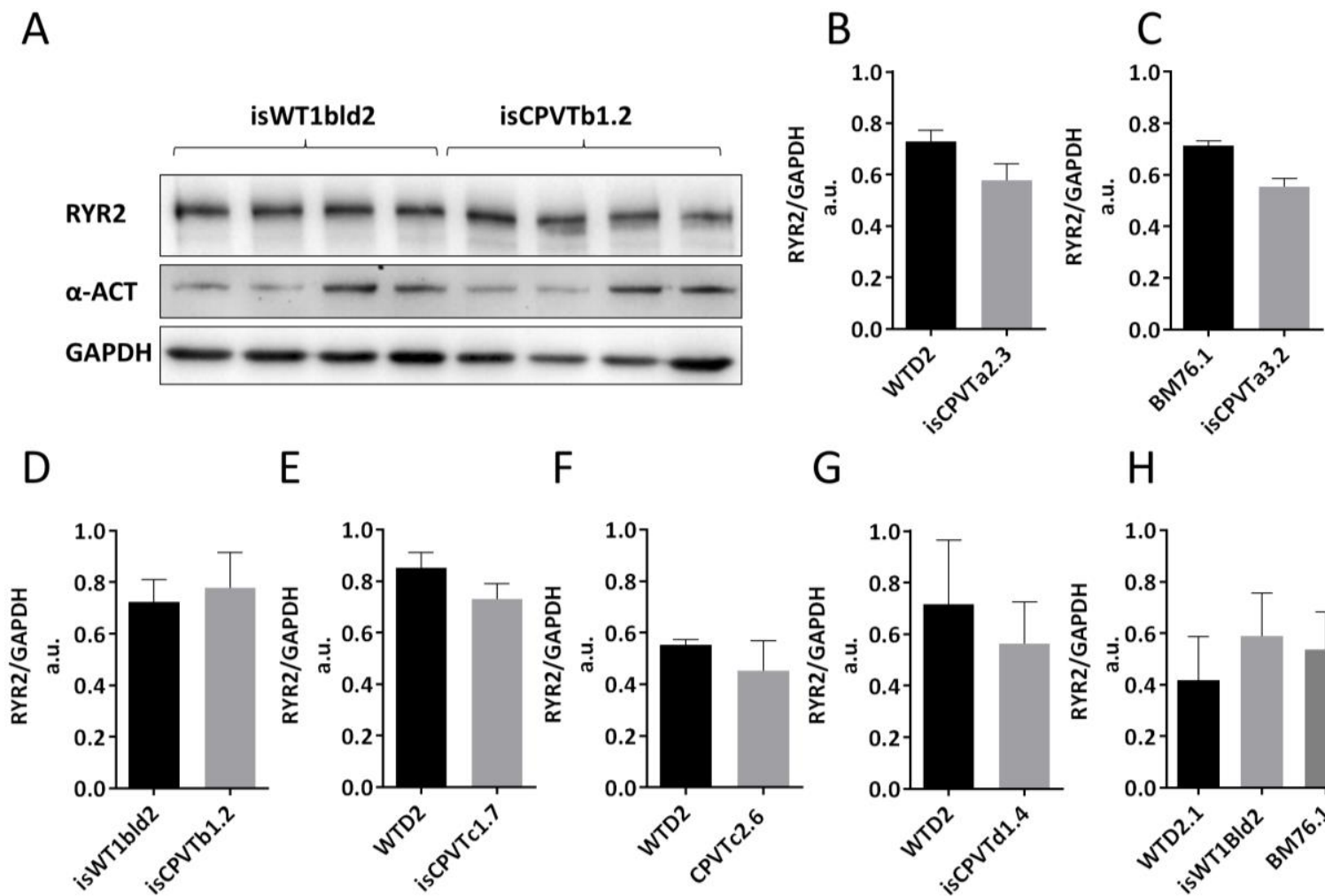

$\mathrm{E}$

$\mathrm{F}$

G

$\mathrm{H}$
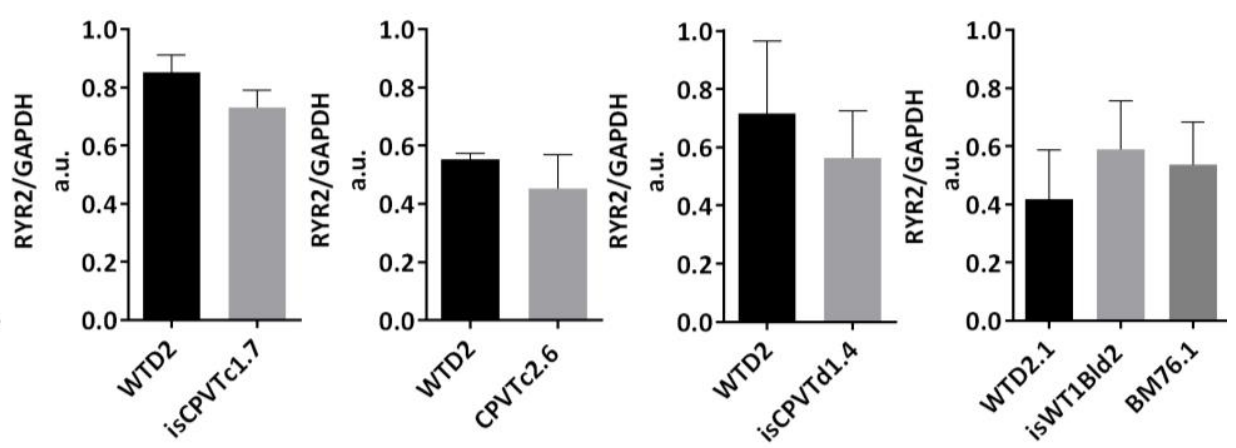

Figure 24. RYR2 expression in CPVT- and CtrI-CMs. Western blot analyses of 3-month-old CMs demonstrated equal protein expression of RYR2 in CPVT-CMs compared to Ctrl-CMs. Detection of the $500 \mathrm{kDa}$ RYR2 protein was done with anti-RYR2 antibodies in combination with HRP-coupled detection antibodies. Figure $\mathrm{A}$ shows a representative western blot of Ctrl- and CPVTb1-CMs expressing RYR2 as well as $\alpha$-ACT. Quantification was done by calculating the signal intensity with Image Lab software. RYR2 was normalized to GAPDH. $\alpha$-ACT was used as positive control for the presence of CMs. Bars represent the mean of 4 samples including SEM.

Western blot analyses were performed to test if CPVT-CMs with RYR2 missense mutations differ in their phosphorylation status of different residues (Ser2808, Ser2814) at basal or under stress conditions in comparison to Ctrl-CMs. The data showed that Ser2808 and Ser2814 are highly phosphorylated at basal conditions in CPVT-CMs as well as in Ctrl-CMs (Fig. 25). After stimulation with isoprenaline, phosphorylation was slightly increased in both CPVT-CMs and Ctrl-CMs. However, mutation-specific differences were not observed, when phosphorylation was compared to Ctrl-CMs. In some cases, phosphorylation of CPVT- and Ctrl-CMs was not increased in response to isoprenaline treatment (Fig. 25). 


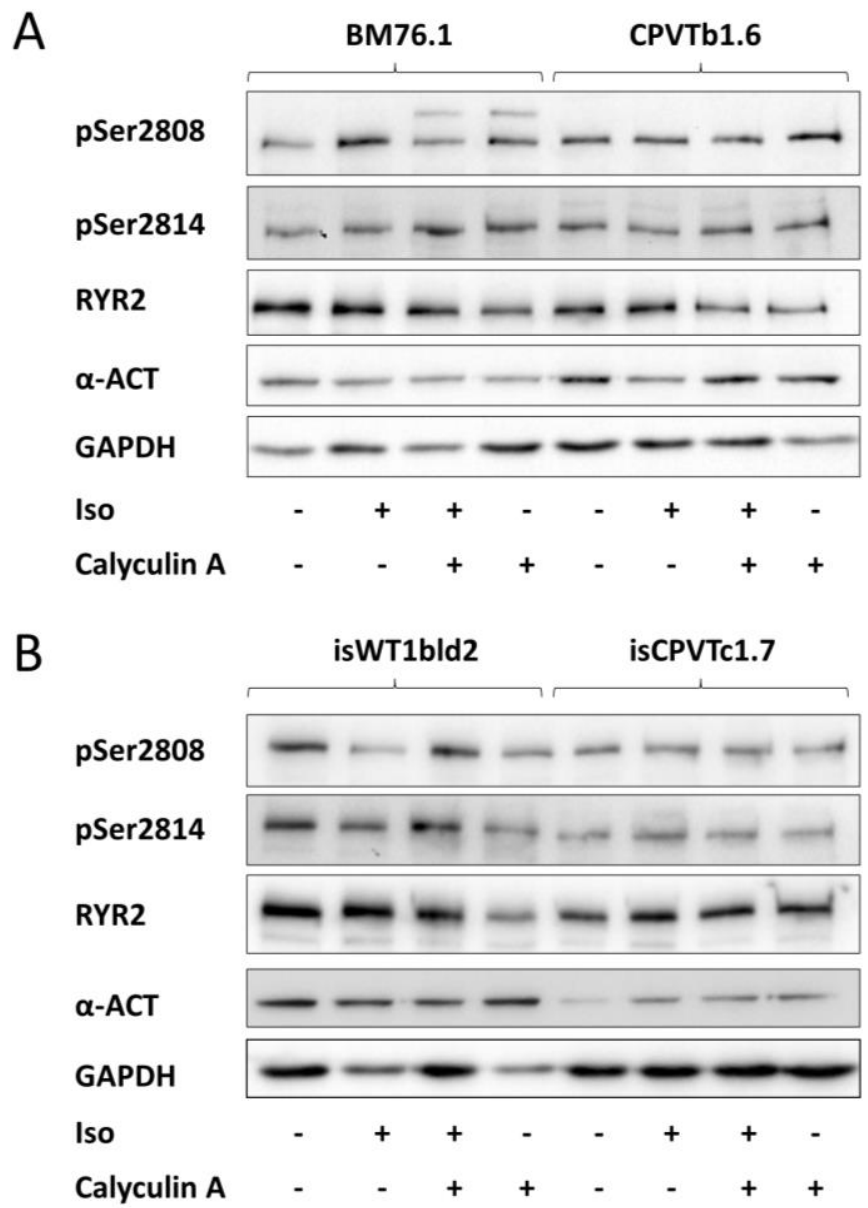

Figure 25. Phospho-RYR2 expression in CPVT- and Ctrl-CMs. CPVT- and Ctrl-CMs were incubated in RPMI without B27 for 2 hours and stimulated with Iso ( $15 \mathrm{~min}, 100 \mathrm{nM}$ ) to induce $\beta$-adrenergic signaling. Simultaneous stimulation with calyculin A (15 $\mathrm{min}, 50 \mathrm{nM})$ should inhibit dephosphorylation of RYR2 during stimulation. Phosphorylation ( $p$ ) was detected with antibodies against RYR2-pSer2808 and RYR2-pSer2814 in combination with HRP coupled detection antibodies. After stimulation with isoprenaline, phosphorylation is slightly increased or stays at the same level in CPVT-CMs and Ctrl-CMs (A, B). Here, representative western blots from patients b1 and c1 compared to Ctrl-CMs are shown (A, B). Mutation- or patient-specific differences were not observed between different CPVT-CMs from patients a2, a3, b1, c1, and c2 compared to Ctrl-CMs.

\subsection{Genome editing by CRISPR/Cas9}

CRISPR/Cas9 genome editing was used as a tool to modify the RYR2 gene in CPVT-hiPSCs. In addition, CPVT-causing mutations should be corrected using ssODNs to generate isogenic controls that might have a potential healthy phenotype. In this study, a protocol for the generation of CRISPR/Cas9-engineered hiPSCs using transfections with sSODNs together with CRISPR/Cas9 plasmids was established. 


\subsubsection{Cleavage assay of CRISPR/Cas9}

When using CRISPR/Cas9 editing tools for the generation of targeted mutations, it is necessary to first determine how efficient Cas9 nucleases cleave the target sequence, which is mostly dependent on the designed gRNA. The genomic region of the RYR2 gene in CRISPR/Cas9 transfected HEK293T cells was amplified and used for the cleavage assay to detect indel (insertion/deletions) introduction (Fig. 26A). The PCR products were analyzed on agarose gel to quantify the intensity of the digested and undigested bands. The plasmid CRISPR-C-1/Cas9 had a calculated cleavage efficiency of $25-27 \%$ and the positive control had a cleavage efficiency around 23-26\% (Fig. 26B).

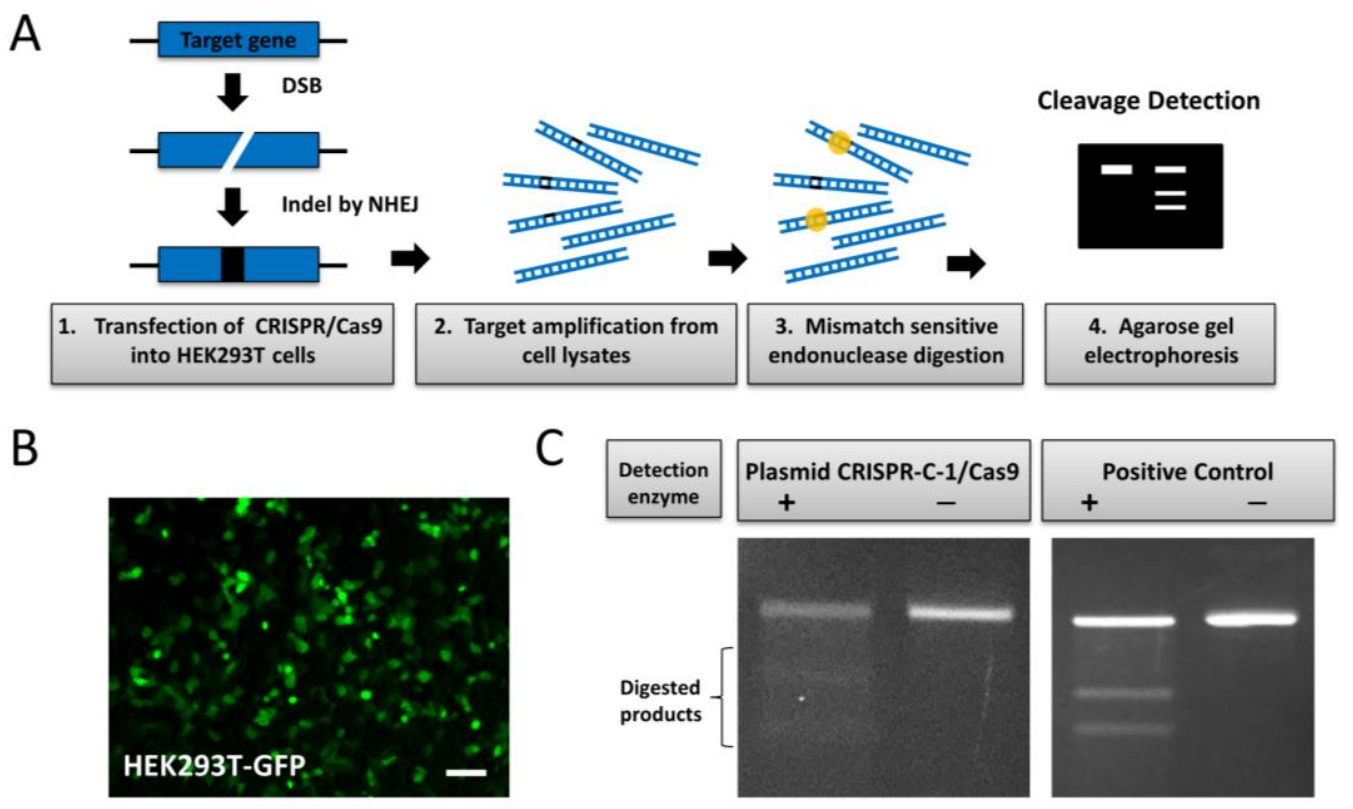

Figure 26. Cleavage assay of CRISPR/Cas9-transfected cells. Schematic presentation of the cleavage assay (A). HEK293T cells were transfected with the CRISPR-C-1/Cas9 plasmid using calcium phosphate-mediated transfection. After $24-48$ hours, the transfected cells are positive for GFP. Scale bar: $100 \mu \mathrm{m}$ (B). DNA lysates from cell pellets were used as direct PCR template for amplification of the CRISPR/Cas9-targeted region of RYR2. The PCR product was denatured and re-annealed to produce heteroduplex mismatches at regions where DSBs had occurred, resulting in indel introduction. These mismatches were recognized and cleaved by the detection enzyme. After gel electrophoreses, the intensity of the digested and undigested bands was analyzed using Alpha Imager software. The cleavage efficiency was calculated according to the formula in the GeneArt Cleavage assay kit. The cleavage efficiency of the plasmid CRISPR-C-1/Cas9 was calculated with $25-27 \%$. The positive control PCR, supplemented in the kit, showed cleavage efficiencies around $23-26 \%$.

\subsubsection{Generation of CRISPR/Cas9-targeted hiPSCs}

For the generation of CRISPR/Cas9-targeted hiPSC clones, hiPSC lines from patients b1, c1 and $\mathrm{c} 2$ were used. CRISPR/Cas9 plasmids and SsODNs were transfected into hiPSCs and 
sorted for $\mathrm{GFP}^{+}$cells (Fig. 27A, B). Designed ssODNs contain silent mutations to destroy the CRISPR binding site and the PAM motif to prevent further cleavage of Cas 9 after HDR. EcoRI restriction site was integrated to enable screening of clones with HDR by restriction digestion (Table 5). To improve the efficiency of precise genome editing with CRISPR/Cas9 for HDR mechanisms, the small molecule L-755,507 was applied for 3 hours before and 24 hours after transfection (Fig. 28A).
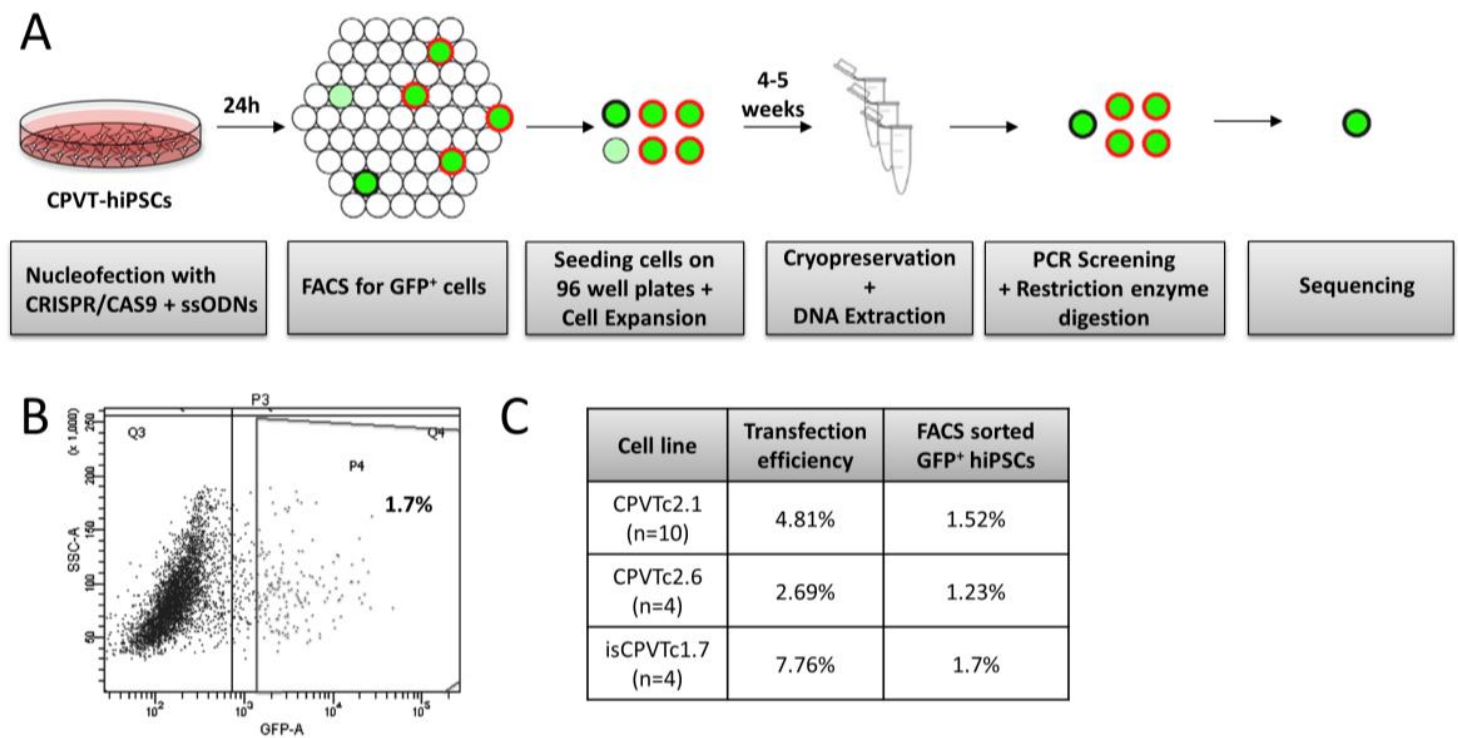

\begin{tabular}{|c|c|c|}
\hline Cell line & $\begin{array}{c}\text { Transfection } \\
\text { efficiency }\end{array}$ & $\begin{array}{c}\text { FACS sorted } \\
\text { GFP }^{+} \text {hiPSCs }\end{array}$ \\
\hline $\begin{array}{c}\text { CPVTc2.1 } \\
(n=10)\end{array}$ & $4.81 \%$ & $1.52 \%$ \\
\hline $\begin{array}{c}\text { CPVTc2.6 } \\
(n=4)\end{array}$ & $2.69 \%$ & $1.23 \%$ \\
\hline $\begin{array}{c}\text { isCPVTc1.7 } \\
(n=4)\end{array}$ & $7.76 \%$ & $1.7 \%$ \\
\hline
\end{tabular}

Figure 27. Strategy for the generation of CRISPR/Cas9-targeted hiPSCs. CRISPR/Cas9 plasmids for gene targeting were introduced to hiPSCs by nucleofection using $2 \times 10^{6}$ cells mixed with $4 \mu \mathrm{g}$ plasmid DNA and $3.3 \mu \mathrm{g}$ ssODNs as donor DNA. In some experiments, L-755,507 (5 $\mu \mathrm{M})$ was added 3 hours before and 24 hours after nucleofection to E8 medium supplemented with TZV. 24 hours after nucleofection, GFP ${ }^{+}$hiPSCs were purified by FACS. $2-7 \times 10^{3}$ cells were seeded onto each Geltrex-coated 96-well plate in a 1:1 mix of E8 and MEF-conditioned E8 medium supplemented with additional hbFGF $(10 \mathrm{ng} / \mathrm{ml}), \mathrm{TZV}, 1 \mathrm{x}$ P/S. After $10-18$ days single colonies were transferred into Geltrex-coated 48-well plates. Confluent cultures of one well of a 48-well plate were transferred onto two wells of a 24-well plate for cryopreservation and DNA extraction. Amplified PCR products of targeted DNA regions were tested for integration of ssODNs by EcoRI restriction digestion. Positive digested cell clones were sequenced (A). Transfection efficiencies of hiPSCs ranged between $2-8 \%(C)$. The percentage of living single cell populations expressing GFP ${ }^{+}$ was much lower (B, C).

Although different transfection methods were tested for hiPSCs, transfection efficiencies of hiPSCs were quite low with around $5 \%$ in average (Fig. 27C). In total, $757 \mathrm{GFP}^{+}$cell clones derived from CPVT-hiPSCs (patients $\mathrm{c} 1$ and $\mathrm{c} 2$ ) were tested with PCR and restriction digestion after cell expansion. In addition, 50 clones were tested from hiPSCs derived from patient b1. Altogether, 22 clones were targeted by the CRISPR/Cas9 system and there were 11 clones integrated with SSODNs in their genome indicated by restriction digestion and sequencing (Fig. 28A). The targeting efficiency of the CRISPR/Cas9 system was about 2.7\%, and the HDR efficiency was about 1.4\%. After treatment with L-755,507, 
the number of clones integrated with SSODNs into their genome was slightly increased. Sequencing data displayed that all CRISPR/Cas9-targeted clones integrated with SSODNs had additional deletions in their genome (Fig. 29). In some cases, mixed hiPSC colonies were observed after cell expansion and sequencing due to high cell densities after sorting (5-15 cells/96-well). These CRISPR/Cas9-targeted clones were purified again using single cell dilution by FACS, expanded and sequenced again. Around $60-70 \%$ of the sorted hiPSC clones maintained normal morphology estimated by visual inspection (Fig. 28B). Some clones differentiated completely into other cell types.

A

\begin{tabular}{|c|c|c|c|c|}
\hline $\begin{array}{c}\text { Used ssODNs + } \\
\text { CRISPR/Cas9 plasmids }\end{array}$ & $\begin{array}{c}\text { Tested } \\
\text { Clones }\end{array}$ & $\begin{array}{c}\text { Edited with } \\
\text { ssODNs }\end{array}$ & $\begin{array}{c}\text { Deletions/ } \\
\text { Insertions* }\end{array}$ & Cell lines \\
\hline CRISPR-Oligo-c & 244 & 2 & 10 & $\begin{array}{c}\text { CPVTc2.1 } \\
\text { CPVTc2.6 }\end{array}$ \\
\hline $\begin{array}{c}\text { CRISPR-Oligo-C-new1 } \\
+ \text { L-755,507 }\end{array}$ & 246 & 7 & 6 & $\begin{array}{c}\text { CPVTc2.1 } \\
\text { CPVTc2.6 }\end{array}$ \\
\hline $\begin{array}{c}\text { CRISPR-Oligo-C-new2 } \\
+ \text { L-755,507 }\end{array}$ & 267 & 1 & 4 & $\begin{array}{c}\text { isCPVTc1.7 } \\
\text { CPVTC2.1 }\end{array}$ \\
\hline CRISPR-Oligo-b & 50 & 1 & 2 & $\begin{array}{c}\text { CPVTb1.6 } \\
\text { CPVTb1.7 }\end{array}$ \\
\hline
\end{tabular}

C

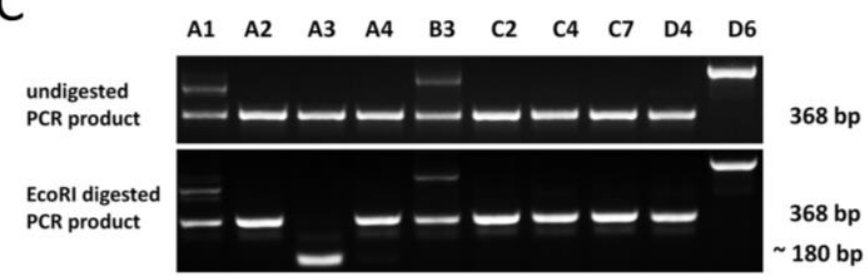

B

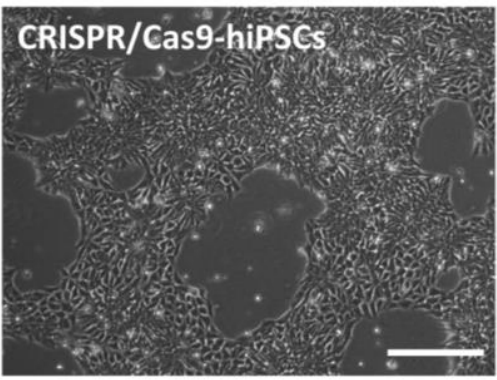

Figure 28. Screening of CRISPR/Cas9-transfected cell clones. In total, 757 clones transfected with plasmid CRISPR-C-1/Cas9 and different ssODNs were screened from hiPSC lines of patients $\mathrm{c} 1$ and c2. 50 clones transfected with plasmid CRISPR-B-1/Cas9 and ssODNs were screened from hiPSC lines of patient b1 (A). Most CRISPR/Cas9-targeted hiPSC clones maintained normal morphology after transfection, sorting and expansion. Scale bar: $300 \mu \mathrm{m}$ (B). Representative PCR shows a positive digested CRISPR/Cas9-targeted clone (A3) as well as CRISPR/Cas9-targeted clones with insertions (A1, B3, D6) indicated by larger fragment sizes (C).

Although fully isogenic corrected CPVT-hiPSCs were not obtained with CRISPR/Cas9 in combination with ssODNs, different RYR2 knockout (KO) clones were generated by using the CRISPR/Cas9 system. The CRISPR/Cas9 targeted clones A3 (RYR2 ${ }^{\varnothing / \varnothing}$-A3) derived from CPVTC2-hiPSCs (Fig. 29A), and X4 (RYR2 $\left.{ }^{\varnothing / \varnothing}-X 4\right)$ generated from CPVTb1-hiPSCs (Fig. 29B) had a homozygous nucleotide deletion which caused a reading frameshift. In RYR2 ${ }^{\varnothing / \varnothing}-A 3$ hiPSCs, a premature termination codon (PTC) occurred at the aa position 4102 in the cytosolic domain of RYR2. In RYR2 ${ }^{\varnothing / \varnothing}$-X4 hiPSCs, a PTC occurred at the aa position 2247 in the cytosolic domain of RYR2. By this way, the truncated proteins generated in RYR2 $2^{\varnothing / \varnothing}$. A3- and RYR2 ${ }^{\varnothing / \varnothing}$-X4-hiPSCs do not have a transmembrane domain and cannot form the channel pore of RYR2. 


\begin{tabular}{|c|c|c|c|c|}
\hline Clone & Origin & ssODNs & L-755,507 & Sequence \\
\hline A3 & CPVTc2.1 & CRISPR-Oligo-c & No & $\begin{array}{l}\text { Allele 1: 5'-GAGAATGAAA - CCTCGACTACGAAGAGTTCGTCAA-3' } \\
\text { Allele 2: 5'-GAGAATGAAA - CCTCGACTACGAAGAGTTCGTCAA-3' }\end{array}$ \\
\hline B6 & CPVTc2.6 & CRISPR-Oligo-C & No & 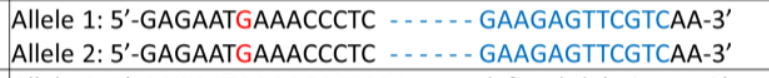 \\
\hline B39 & CPVTc2.6 & CRISPR-Oligo-c & No & $\begin{array}{l}\text { Allele 1: 5'-GAGAATGAAACCCTCGA- - -undefined deletion- - - } 3^{\prime} \\
\text { Allele 2: 5'-GAGAATAAAACCCTCGA- - -undefined deletion- - -3' }\end{array}$ \\
\hline N9 & CPVTc2.6 & $\begin{array}{l}\text { CRISPR-Oligo-c- } \\
\text { new1 }\end{array}$ & Yes & $\begin{array}{l}\text { Allele 1: 5'-GAGAATGAAACTCTCGACTACGAAGAATTCGTCAA-3' } \\
\text { Allele 2: 3'-GAGAATGAAACTCTCGACTACGAAGAATTCGTCAA-5' }\end{array}$ \\
\hline N14 & CPVTc2.6 & $\begin{array}{l}\text { CRISPR-Oligo-c- } \\
\text { new1 }\end{array}$ & Yes & $\begin{array}{l}\text { Allele 1: 5'-GAGAATGAAACTCTCGACTACGAAGAATTCGTCAA-3' } \\
\text { Allele 2: 5'-GAGAATAAAACCCTCGACTACGAAGAGTTCGTCAA-3' }\end{array}$ \\
\hline P10 & CPVTc2.6 & $\begin{array}{l}\text { CRISPR-Oligo-c- } \\
\text { new1 }\end{array}$ & Yes & $\begin{array}{l}\text { Allele 1: 5'-GAGAATGAAACCCTCGA- - -undefined deletion- - - } 3^{\prime} \\
\text { Allele 2: 5'-GAGAATAAAACCCTCGA- - - undefined deletion- - - } 3^{\prime}\end{array}$ \\
\hline P39 & CPVTc2.6 & $\begin{array}{l}\text { CRISPR-Oligo-c- } \\
\text { new1 }\end{array}$ & Yes & $\begin{array}{l}\text { Allele 1: 5'-GAGAATGAAACTCTCGACTACGAAGAATTCGTCAA-3' } \\
\text { Allele 2: 5'-GAGAATGAAACCCTCGA- }\end{array}$ \\
\hline 033 & CPVTc2.1 & $\begin{array}{l}\text { CRISPR-Oligo-c- } \\
\text { new1 }\end{array}$ & Yes & $\begin{array}{l}\text { Allele 1: 5'-GAGAATAAAACTCTCGACTACGAAGAATTCGTCAA-3' } \\
\text { Allele 2: 5'-GAGAATGAAACCCTCGACTACGAGAAATTCGTCAA-3' }\end{array}$ \\
\hline 0120 & CPVTc2.1 & $\begin{array}{l}\text { CRISPR-Oligo-c- } \\
\text { new1 }\end{array}$ & Yes & $\begin{array}{l}\text { Allele 1: 5'-GAGAATGAAACTCTCGACTACGAAGAATTCGTCAA-3' } \\
\text { Allele 2: 5'-GAGAATAAAACCCTCGACTACGAAGAGTTCGTCAA-3' }\end{array}$ \\
\hline T42 & isCPVTc1.7 & $\begin{array}{l}\text { CRISPR-Oligo-c- } \\
\text { new2 }\end{array}$ & Yes & $\begin{array}{l}\text { Allele 1: 5'-GAGAATGAAACCCTCGACTACGAAGAGTTCGTCAA-3' } \\
\text { Allele 2: 5'-G-G- }-\ldots \text { - }\end{array}$ \\
\hline & & & & \\
\hline Clone & Origin & ssODNs & L-755,507 & Sequence \\
\hline X4 & CPVTb1.6 & CRISPR-Oligo-b-1 & No & $\begin{array}{l}\text { Allele 1: 5'-GAGAGGTTCA ACACCA- - - - -ATGTGGCTGCAGCTTCGG } \\
\text { TGATGGATAATAATGAACTAGCATTAG-3' } \\
\text { Allele 2: 5'-GAGAGGTTCA ACACCA- - - - -ATGTGGCTGCAGCTTCGG } \\
\text { TGATGGATAATAATGAACTAGCATTAG-3' }\end{array}$ \\
\hline $\mathrm{X} 2$ & CPVTb1.6 & CRISPR-Oligo-b-1 & No & $\begin{array}{l}\text { Allele 1: 5'-GCCT CCCCAGCTATGAGAGGTTCA ACACC- - - - - -3' } \\
\text { Allele 2: 5'-GCCT CCCCAGCTATGAGAGGTTCA ACACC3' }\end{array}$ \\
\hline
\end{tabular}

Figure 29. Sequencing of CRISPR/Cas9-targeted clones. CRISPR/Cas9-targeted cell clones were sequenced to test if ssODNs were integrated into the genome after EcoRI-digested fragments were observed. Depending on the used ssODNs for transfections, cell clones had silent mutations after successful HDR (purple, A). Clone A3 had a homozygous deletion of a single bp (A). Clone X4 had a homozygous deletion of 4 bps (B). Clone B6 had a homozygous deletion of two triplets (A). Clone P39 had a heterozygous deletion of 5 triplets in one allele and the ssODNs integrated in the second allele. The clones $\mathrm{N} 14, \mathrm{O} 33$ and $\mathrm{O} 120$ had a heterozygous integration of ssODNs in the healthy allele. Clone T42 had the ssODNs integrated in the CPVT causing allele, but with one additional 17 bp deletion (A).

Another CRISPR/Cas9-edited cell clone T42, defined as RYR2 ${ }^{+/ \varnothing}-\mathrm{T} 42$-hiPSCs, integrated the ssODNs in combination with a 17 bp deletion into the mutated CPVT allele resulting in a PTC at the aa position 4075 in the cytosolic domain of RYR2. The sequence of the healthy


protein generated from the mutant allele does not have a transmembrane domain and cannot be used for the channel formation. The full-length protein generated from the healthy allele can form a functional channel of RYR2. To further study the function of RYR2 and to test whether the knockout of the RYR2 mutant allele could rescue the disease phenotype, the homozygous and heterozygous CRISPR/Cas9-edited lines

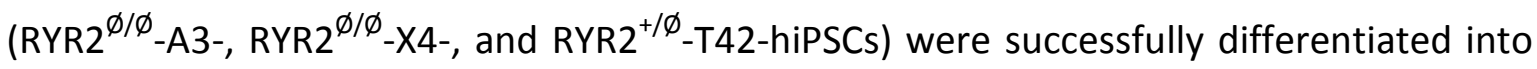
beating CMs using our standard directed differentiation protocol and were cultivated up 
to three months. After three-month cultivation, CRISPR/Cas9-edited CMs (RYR2 ${ }^{\varnothing / \varnothing}-A 3$ and $\mathrm{RYR}^{+/ \varnothing}{ }_{-\mathrm{T} 42)}$ do not show differences in RYR2 expression on mRNA level in comparison with their corresponding original CPVT-CMs (Fig. 30A, B). Moreover, CRISPR/Cas9-edited $\mathrm{CMs}\left(\mathrm{RYR} 2^{\varnothing / \varnothing}-\mathrm{A} 3\right.$ and $\mathrm{RYR} 2^{+/ \phi}-\mathrm{T} 42$ ) express cardiac-specific genes regulating $\mathrm{Ca}^{2+}$ signaling, such as the cardiac inositol 1,4,5-triphosphate receptor types 1 and 2 (IP3R1, IP3R2) and the calcium voltage-gated channel subunit alpha-1C (CACNA1C), as well as genes coding for sarcomeric proteins, such as CTNT and $\alpha-A C T$. However, no tendency of increased/or decreased expression of the tested genes in CRISPR/Cas9-edited CMs was observed in comparison to their corresponding CPVT-CMs (Fig. 30).

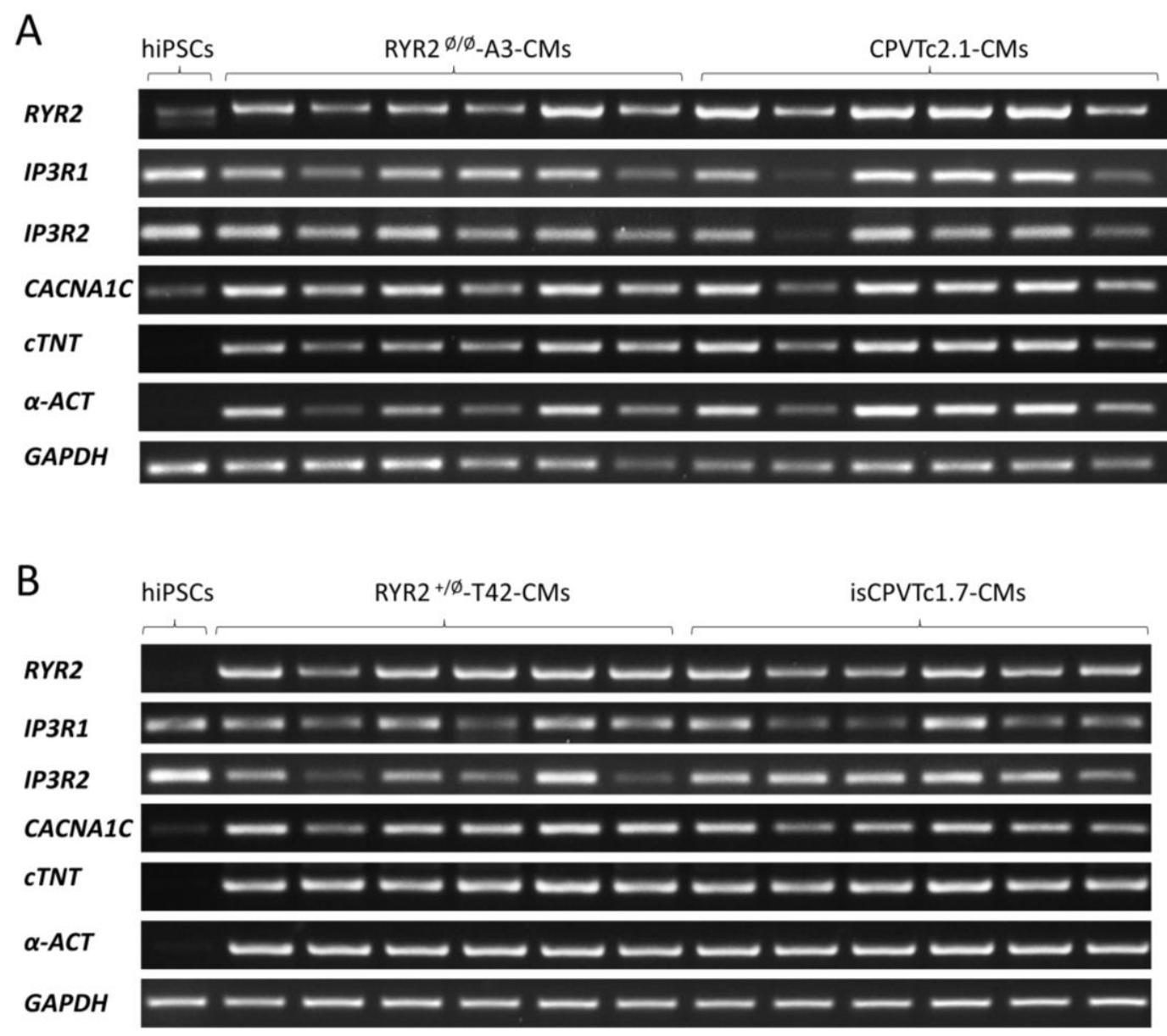

Figure 30. Gene expression of $\mathrm{Ca}^{2+}$ regulating genes in CRISPR/Cas9-edited CMs. $\mathrm{RYR}^{\varnothing / \varnothing}{ }_{-} \mathrm{A} 3-$, RYR2 $^{+/ \phi}$-T42-hiPSCs and the original CPVT-hiPSCS (CPVTc2.1, isCPVTc1.7) were directly differentiated into CMs. After 3-month cultivation, mRNA was isolated from independent cultivated $\mathrm{CMs}$ and reverse transcribed into CDNA. RYR2 ${ }^{\varnothing / \phi}-\mathrm{A} 3-\mathrm{CMs}$ as well as CPVTC2.1-CMs express $\mathrm{Ca}^{2+}$ regulatory genes, such as IP3R1, IP3R2 and CACNA1C, at the same level (A). Similar results were observed in RYR2 ${ }^{+/ \phi}-\mathrm{T} 42-\mathrm{CMs}$ that were compared to their corresponding origin of isCPVTC1.7-CMs (B). HiPSCs were used as negative control for the cardiac-specific marker genes cTNT and $\alpha-A C T$. GAPDH was used as reference gene. 
The next experiment tested if a heterogeneous PTC in the $R Y R 2$ gene leads to a different

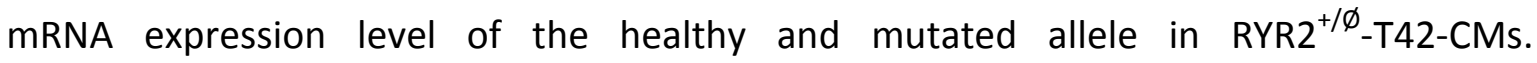
Interestingly, allele-specific sequencing of the heterozygous RYR2 ${ }^{+/ \varnothing}-\mathrm{T} 42-\mathrm{CMs}$ showed that the mRNA expression of the disrupted allele is highly decreased (11.4\%) in comparison to the wildtype/healthy allele (88.6\%) (Fig. 31). This result highlights that we generated a CRISPR/Cas9-targeted hiPSC clone expressing almost only the healthy allele.

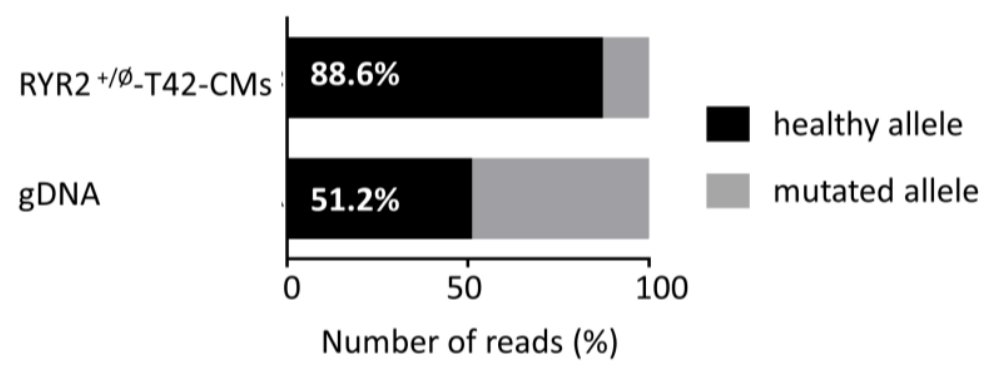

Figure 31. Allele-specific RYR2 expression in RYR2 ${ }^{+/ \phi}-\mathrm{T42}-\mathrm{CMs}$. Sequencing of cDNA copies showed that 167 of 1280 reads had a 17 bp deletion (11.4\%). All reads containing the $17 \mathrm{bp}$ deletion had three additional nucleotide substitutions in the CRISPR/Cas9-targeted CPVT-allele originated from the introduced ssODNs. $88.6 \%$ of the CDNA copies showed no deletion or substitution in comparison to the reference sequence $(n=1)$. Sequencing of gDNA showed similar copy numbers of the healthy allele compared to the mutated one.

Western blot analysis using the rabbit antibody that can detect both the full length and truncated RYR2 proteins showed no change in the full-length RYR2 in RYR2 ${ }^{+/ \varnothing}-$ T42-CMs $^{-}$ compared to CPVT-CMs (Fig. 32). The truncated protein with a predicted size of $448 \mathrm{kDa}$ was not detected in RYR2 ${ }^{+/ \phi}-\mathrm{T}_{42}-\mathrm{CM}$ (Fig. 32A). In contrast, RYR2 ${ }^{\varnothing / \phi_{-A}}$ - and RYR2 ${ }^{\varnothing / \phi_{-X}}$ showed no detectable RYR2 protein by western blot analysis in comparison to CPVT-CMs (Fig. 32C, D). 
A

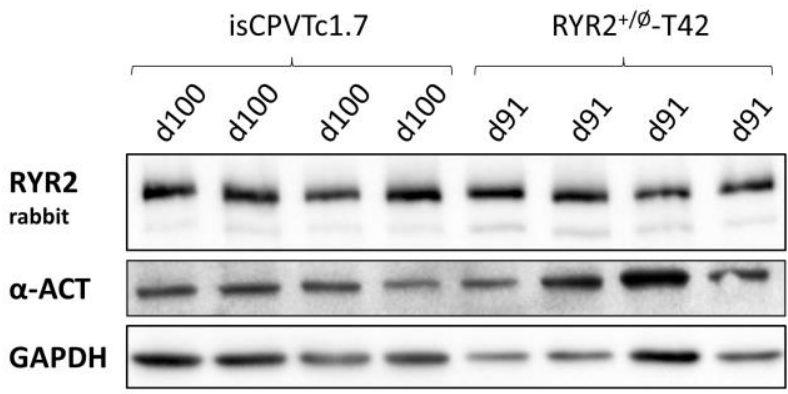

B

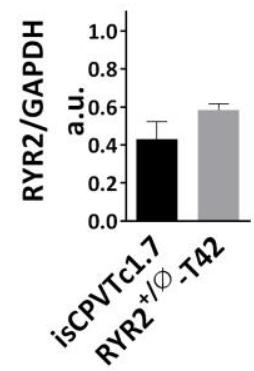

C

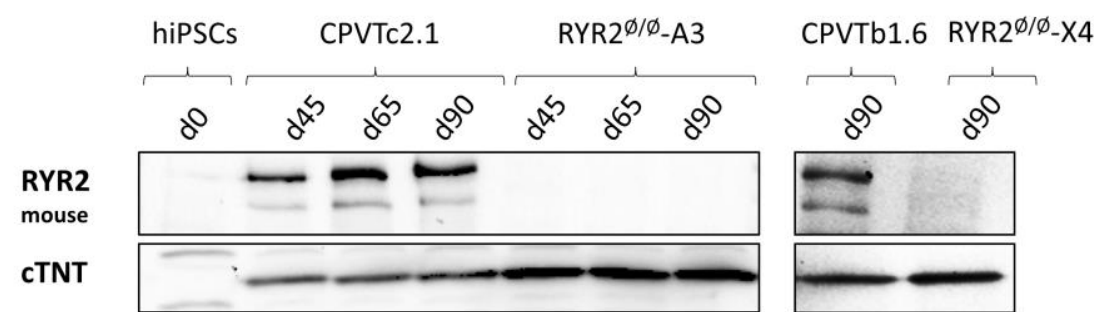

D

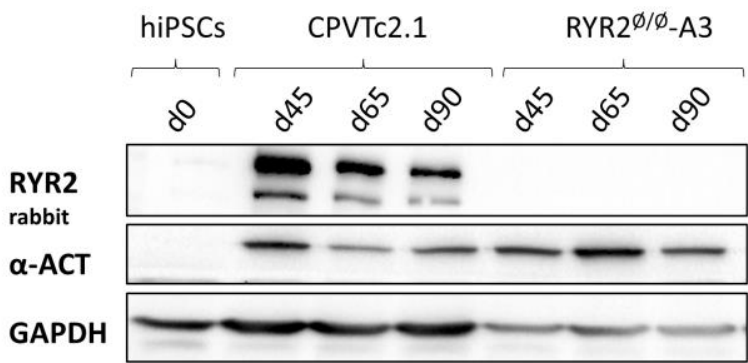

Figure 32. RYR2 expression in CRISPR/Cas9-edited CMs. RYR2 ${ }^{+/ \varnothing}-\mathrm{T} 42-\mathrm{CM}$ s showed no visible change in RYR2 expression compared to CPVT-CMs (A). Analysis of western blots $(n=4)$ showed a slight, but not significant, increase of RYR2 in RYR2 ${ }^{+/ \phi}$-T42-CMs compared to isCPVTc1.1-CMs (B). Western blot analysis of RYR2 ${ }^{\phi / \phi}$-A3-CMs showed no detectable RYR2 expression (C, D). Two different RYR2 ${ }^{\Phi / \phi}$ cell lines from different hiPSC origin $(A 3, X 4)$ were tested with two different RYR2 antibodies recognizing the full length protein ( $\alpha$-RYR2 mouse) (C) and the $N$-terminal region ( $\alpha$-RYR2 rabbit) (D) in front of the PTC. CTNT and $\alpha$-ACT were used as positive controls for the presence of CMs in protein lysates. GAPDH was used as loading control.

Furthermore, immunofluorescence staining with the anti- $\alpha$-actinin antibody revealed the $z$-line zone in a typical striated pattern in RYR2 ${ }^{\varnothing / \varnothing}$-A3- (Fig. 33D) and RYR2 $2^{\varnothing / \varnothing}-\mathrm{X} 4-\mathrm{CMs}$ (Fig. 33G) similar to CPVTc2-CMs (Fig. 33A). Whereas RYR2 was co-localized with $\alpha$-ACT in the z-line zone in CPVTC2-CMs (Fig. 33C), RYR2 protein was not detected in a striated pattern in RYR2 ${ }^{\varnothing / \varnothing}-A 3$ - and RYR2 ${ }^{\varnothing / \varnothing}-X 4-C M s$ (Fig. 33F, I). 


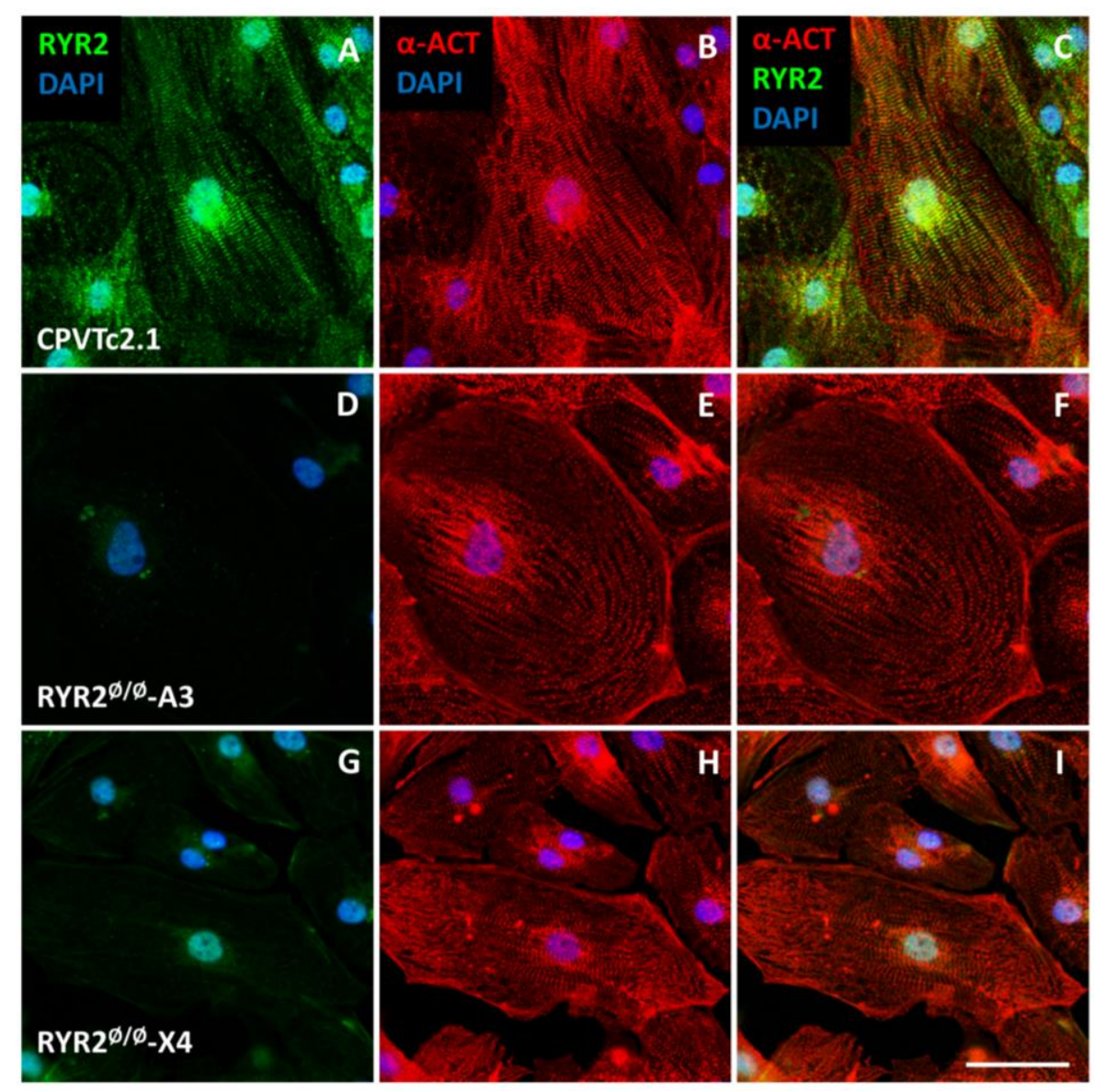

Figure 33. Immunostaining of RYR2 ${ }^{\Phi / \varnothing}$-A3-CMs in comparison to CPVTC2.1-CMs. Two month old CMs were stained with antibodies against RYR2 (A, D, G, green). RYR2 ${ }^{\phi / \phi}-A 3-C M s$ did not express RYR2 but had normal sarcomeric structures indicated by staining of $\alpha-A C T$ (D, G). CPVTC2.1-CMs showed a striated pattern of RYR2 (A, green) which co-localized with the expression of $\alpha$-ACT (B, red). Cell nuclei were stained with DAPI (blue). Scale bar: $50 \mu \mathrm{m}$.

To test if the degradation of the truncated versions of RYR2 might occur in RYR2 ${ }^{\varnothing / \varnothing}$-A3and RYR2 ${ }^{+/ \varnothing}$-T42-CMs, 3-month-old CMs were treated with the proteasome and calpain inhibitor MG132 (24 h, $10 \mu \mathrm{M})$ together with the autophagosome-lysosome fusion inhibitor bafilomycin A1 (BafA1) (6 h, $100 \mathrm{nM})$. However, no truncated proteins were observed in both RYR2 ${ }^{\varnothing / \varnothing}-A 3-C M s$ (Fig. 34A) and RYR2 ${ }^{+/ \varnothing}-$ T42-CMs (Fig. 34B) after the inhibition of protein degradation. Moreover, no changes in expression of the full-length RYR2 were observed in RYR2 ${ }^{+/ \varnothing}-$ T42-CMs compared to CPVTC1-CMs (Fig. 34). 


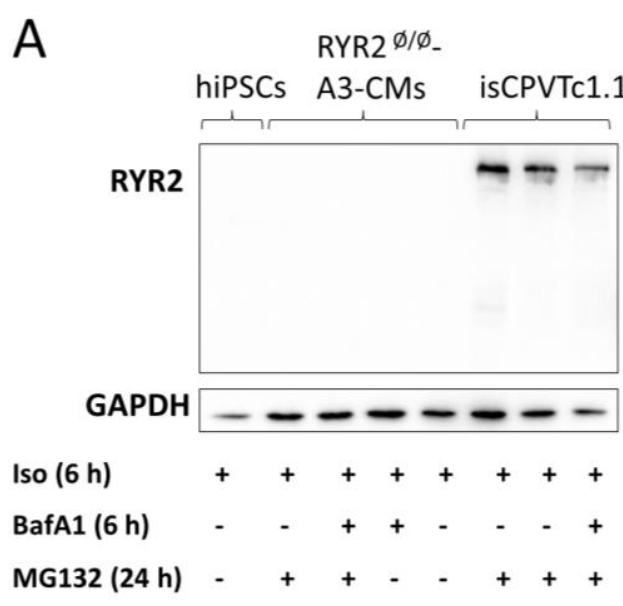

B



Figure 34. Analysis of RYR2 protein degradation in CRISPR/Cas9-edited CMs. RYR2 ${ }^{\varnothing / \phi}-A 3-C M s$, RYR2 $^{+/ \phi}$-T42-CMs and CPVTC1-CMs stimulated with Iso (100 nM, 6 h) were stepwise treated with the proteasome and calpain inhibitor MG132 $(10 \mu \mathrm{M}, 24 \mathrm{~h})$, and the autophagy inhibitor BafA1 $(100 \mathrm{nM}, 6 \mathrm{~h})$ to inhibit potential protein degradation. Truncated versions of RYR2 were not observed in both RYR2 ${ }^{\varnothing / \varnothing}-\mathrm{A} 3$ - and RYR2 ${ }^{+/ \phi}-\mathrm{T}_{4} 2-\mathrm{CM}$ s after single and combined treatment with inhibitors (A, B). In addition, the amount of the full-length RYR2 protein did not change in RYR2 ${ }^{+/ \varnothing}$. T42-CMs ( $n=1,2$ blots). GAPDH was used as loading control.

To analyze potential compensatory mechanism in $\mathrm{RYR}^{\varnothing / \varnothing}-\mathrm{A} 3-\mathrm{CM}$, the expression of $\mathrm{Ca}^{2+}$ regulating proteins were studied. However, RYR2 ${ }^{\varnothing / \varnothing}-A 3-C M s$ did not show any changes in protein expression of IP3R, SERCA2A or NCX1 in comparison to CPVT-CMs (Fig. 35).

A

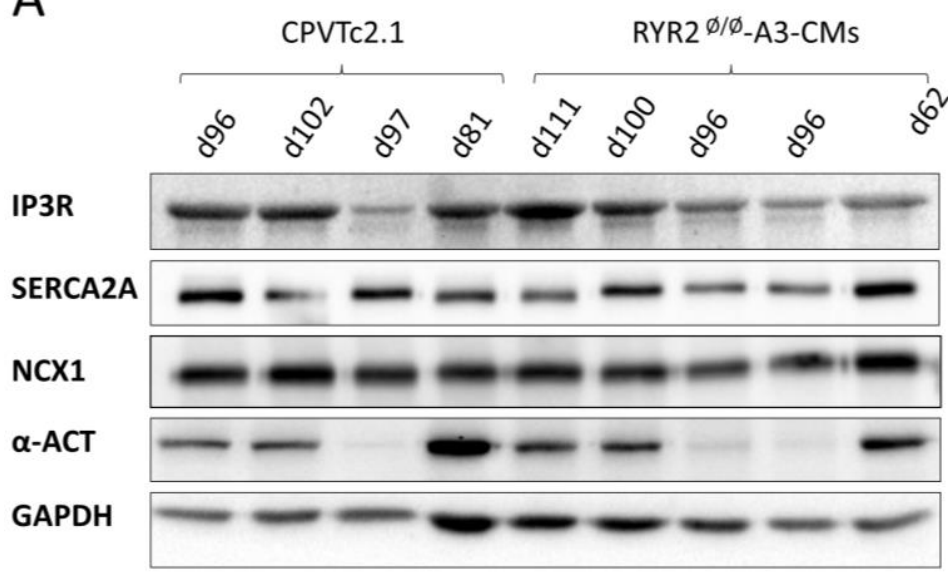

B



C

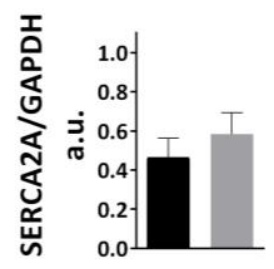

D

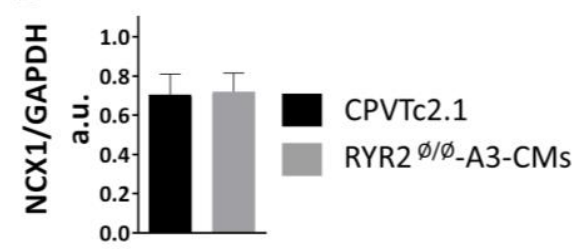

Figure 35. Expression of $\mathrm{Ca}^{2+}$ regulatory proteins in $\mathrm{RYR2}^{\varnothing / \varnothing}-\mathrm{A3}-\mathrm{CMs}$. Western blot analysis showed that IP3R, SERCA2a and NCX1 are not differently expressed in 2-3-month-old RYR2 ${ }^{\varnothing / \varnothing}$-A3CMs compared to CPVT-CMs. $\alpha$-ACT was used as positive control for the presence of CMs in the protein lysates. GAPDH was used as loading control.

In the next step, the $\mathrm{Ca}^{2+}$ handling in RYR2 ${ }^{+/ \phi}-\mathrm{T}_{42}-\mathrm{CM}$ s and RYR2 ${ }^{\varnothing / \varnothing}-\mathrm{A} 3-\mathrm{CM}$ s was analyzed in comparison to CPVTc1/c2-CMs (cell line: CPVTc1.7, CPVTc2.1) and Ctrl-CMs (cell line: isWT1bld2) under isoprenaline stimulated conditions (Fig. 36A-F). 
A

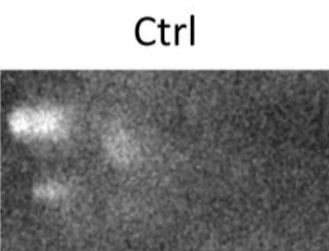

CPVTc1/c2

RYR2 $^{+/ \phi}-$ T42 $^{-}$

RYR2 ${ }^{\phi / \phi}-\mathrm{A} 3$
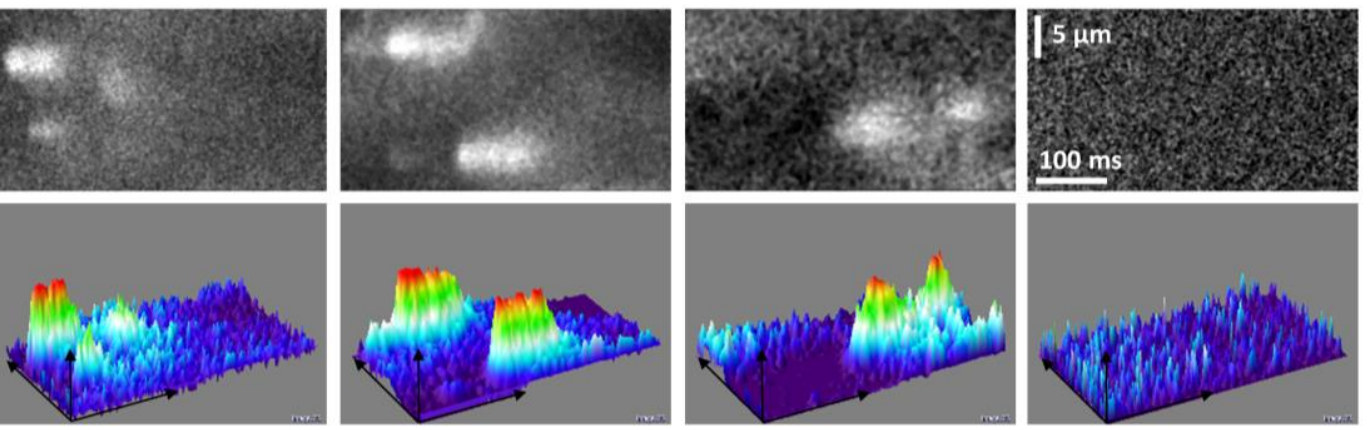

B

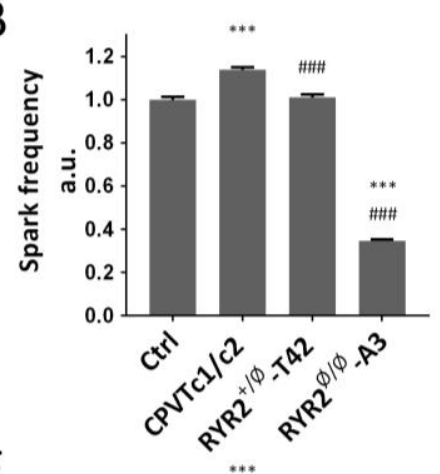

C
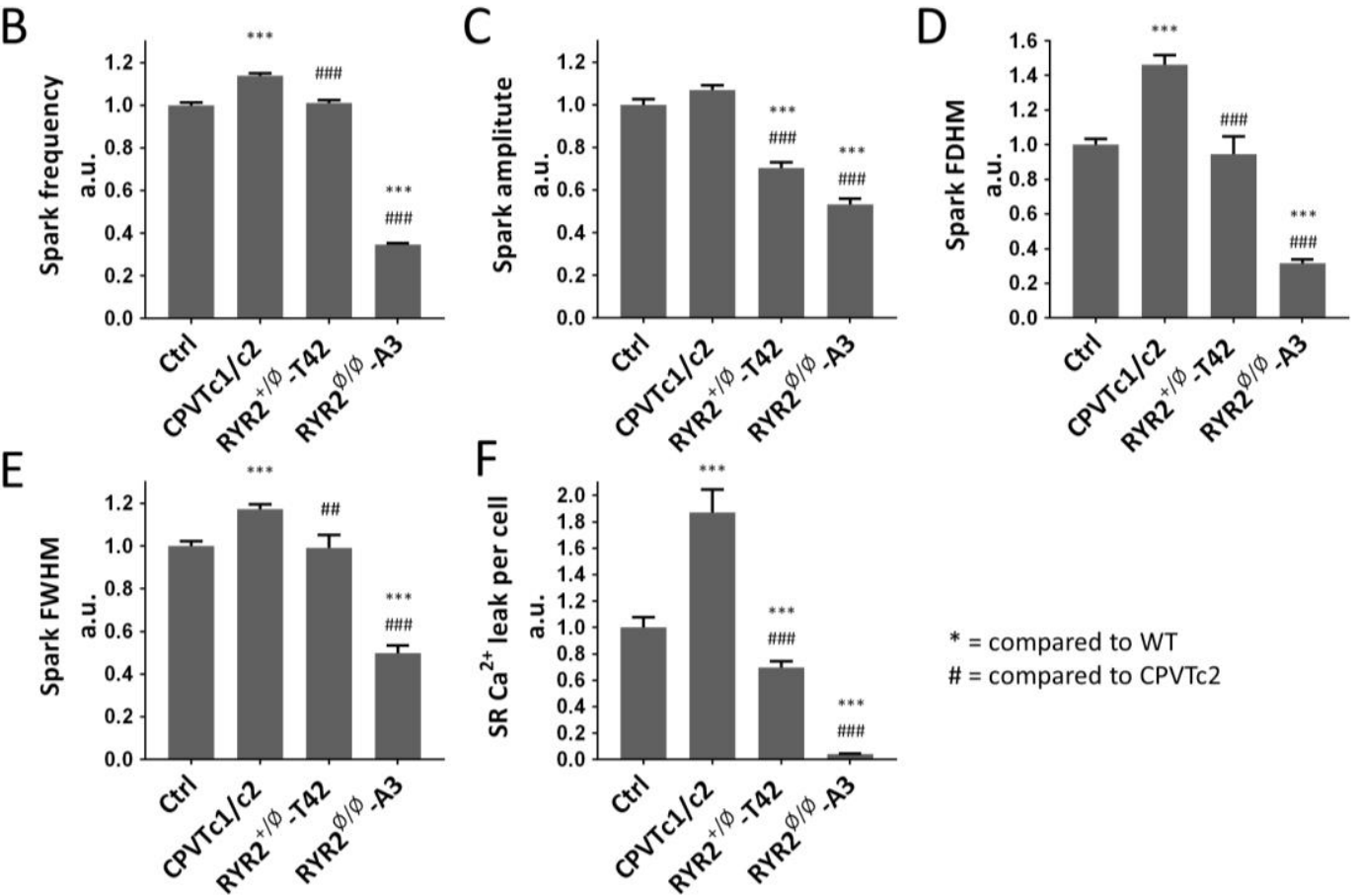

$*$ = compared to WT

$\#$ = compared to CPVTc2

Figure 36. Isoprenaline-induced $\mathrm{Ca}^{2+}$ sparks in CRISPR/Cas9-engineered CMs. Ctrl-CMs ( $\left.n=358\right)$,

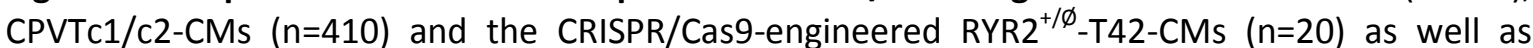
RYR2 ${ }^{\Phi / \varnothing}$-A3-CMs $(n=21)$ were used for cytosolic calcium recordings by confocal line scan imaging. All CMs (d90-108) loaded with fluo-4/AM and stimulated with isoprenaline (100 nM) were paced with $0.25 \mathrm{~Hz}$. Example recordings from Ctrl-, CPVTc1/c2-, and RYR2 ${ }^{+/ \varnothing}-\mathrm{T}_{42}-\mathrm{CMs}$ show $\mathrm{Ca}^{2+}$ sparks visualized by $3 \mathrm{D}$ reconstruction of selected areas after stimulation with isoprenaline. In contrast, $\mathrm{RYR2}^{\phi / \phi}-\mathrm{A} 3-\mathrm{CMs}$ did not display $\mathrm{Ca}^{2+}$ sparks after stimulation with isoprenaline (A). After stimulation with isoprenaline, RYR2 $2^{+/ \phi}-\mathrm{T}_{42}-\mathrm{CMs}$ and $\mathrm{RYR} 2^{\varnothing / \phi}-\mathrm{A} 3-\mathrm{CMs}$ display highly significant reduced $\mathrm{Ca}^{2+}$ spark frequency (B), with significantly lower spark amplitude (C) lower FDHM (D), lower FWHM (E), and reduced SR $\mathrm{Ca}^{2+}$ leak per cell (F) compared to CPVTc1/c2-CMs. In comparison to Ctrl-CMs, RYR2 ${ }^{+/ \varnothing}-\mathrm{T} 42-\mathrm{CMs}$ did not show differences in regard to spark frequency (A), FDHM (D), and FWHM (E), but lower spark amplitude (C), and reduced SR $\mathrm{Ca}^{2+}$ leak per cell (F). All Measurements were shown in relation to the Ctrl-CMs that were set to 1 . Data are shown as mean $\pm S E M$, two-tailed student's $t$ test with two samples and unequal variance was used for the statistical analysis. ${ }^{* *}, \mathrm{p}<0.001 ;{ }^{* *}, \mathrm{p}<0.01 ;{ }^{*}, \mathrm{p}<0.05 ; \mathrm{n} . \mathrm{s} ., \mathrm{p}>0.05$ (in cooperation with Karolina Sekeres). 
The analysis of $\mathrm{Ca}^{2+}$ leak showed that $\mathrm{RYR}^{+/ \varnothing}-\mathrm{T} 42-\mathrm{CM}$ resemble a phenotype that is comparable with Ctrl-CMs in respect to the spark frequency (Fig. 36B), FDHM (Fig. 36D), and FWHM (Fig. 36E). Furthermore, RYR2 ${ }^{+/ \phi}-\mathrm{T} 42-\mathrm{CMs}$ displayed significantly lower $\mathrm{Ca}^{2+}$ spark frequency, lower spark amplitude, reduced FWHM, lower FDHM and reduced SR $\mathrm{Ca}^{2+}$ leak per cell compared to CPVTc1/c2-CMs (Fig. 36). RYR2 ${ }^{\varnothing / \varnothing}-\mathrm{A} 3-\mathrm{CMs}$ showed highly significant reduced spark frequency, spark amplitude, FWHM, FDHM and SR $\mathrm{Ca}^{2+}$ leak in comparison to Ctrl- and CPVTC1/C2-CMs (Fig. 36B-F). For example, RYR2 ${ }^{\varnothing / \varnothing}-\mathrm{A} 3-\mathrm{CMs}$ display $65 \%$ reduced spark frequency as well as $96 \%$ reduced SR $\mathrm{Ca}^{2+}$ leak is in comparison to Ctrl-CMs (Fig 36B, F).

In the next experiments, fluo-4/AM-loaded RYR2 ${ }^{\varnothing / \varnothing}-A 3-, R^{2} R 2^{+/ \varnothing}-T_{42}-C M s$ and CPVT-CMs were paced with $0.25 \mathrm{~Hz}$ and $\mathrm{Ca}^{2+}$ transients were studied. The data showed that shapes of $\mathrm{Ca}^{2+}$ transients in RYR2 ${ }^{+/ \varnothing}-\mathrm{T42}-\mathrm{CMs}$ are comparable with those in CPVTC2-CMs (37A, B). However, the amplitude of $\mathrm{Ca}^{2+}$ transients is slightly smaller in $\mathrm{RYR}^{+/ \varnothing}-\mathrm{T}_{42}-\mathrm{CMs}$ compared to CPVTC2-CMs. By contrast, the shapes of $\mathrm{Ca}^{2+}$ transients in RYR2 ${ }^{\varnothing / \varnothing}$-A3-CMs are clearly different from those in in RYR2 ${ }^{+/ \varnothing}-\mathrm{T}_{42}-\mathrm{CM}$ s and CPVTC2-CMs. The amplitude of $\mathrm{Ca}^{2+}$ transients in RYR2 ${ }^{\varnothing / \varnothing}-\mathrm{A} 3-\mathrm{CM}$ is much smaller when compared to CPVTC2-CMs and $\mathrm{RYR2}^{+/ \varnothing}-\mathrm{T} 42-\mathrm{CMs}$ (Fig. 37A-C).

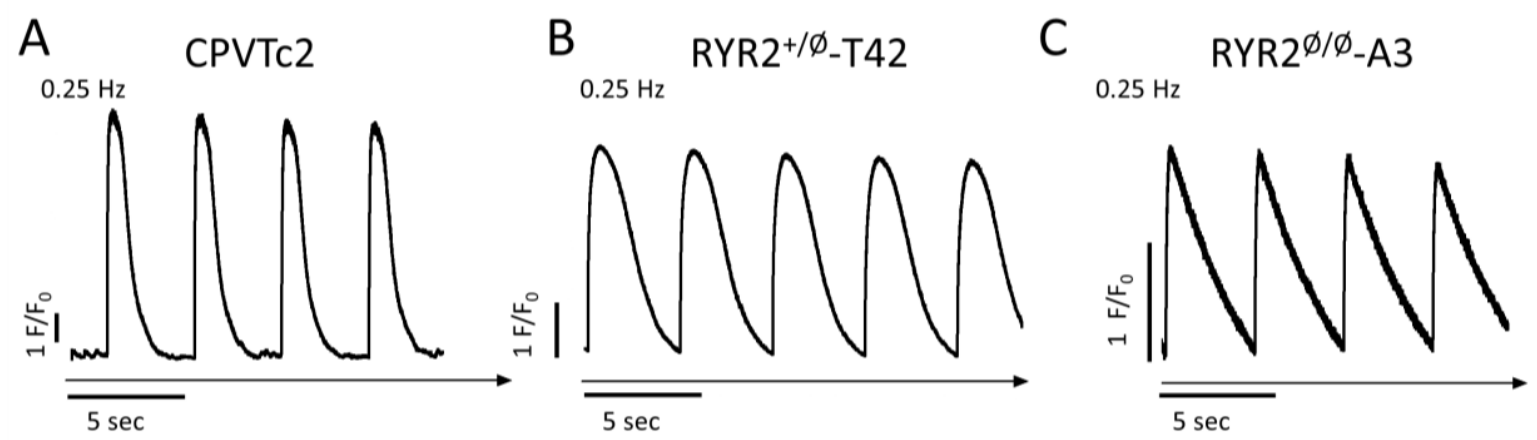

Figure 37. $\mathrm{Ca}^{2+}$ transients from CRISPR/Cas9-engineered CMs. For the measurement of $\mathrm{Ca}^{2+}$ transients, fluo-4/AM loaded CPVTC2-CMs, RYR2 ${ }^{+/ \varnothing}-\mathrm{T}_{42}-\mathrm{CMs}$ and $\mathrm{RYR2}{ }^{\varnothing / \varnothing}-\mathrm{A} 3-\mathrm{CM}$ s were paced with $0.25 \mathrm{~Hz}$. Confocal line scan imaging was performed and representative $\mathrm{Ca}^{2+}$ transients of CPVTC2-CMs (A), RYR2 ${ }^{+/ \phi}-\mathrm{T} 42-\mathrm{CMs}$ (B) and RYR2 ${ }^{\phi / \phi}-\mathrm{A} 3-\mathrm{CMs}$ (C) were shown. In comparison to CPVTC2-CMs and RYR2 ${ }^{+/ \phi}-\mathrm{T} 42-\mathrm{CMs}$, the amplitudes of the $\mathrm{Ca}^{2+}$ transients of $\mathrm{RYR}^{\varnothing / \phi}-\mathrm{A} 3-\mathrm{CM}$ s were much smaller and transient decreased much slower to the baseline (C). F/FO: fluorescence (F) normalized to baseline fluorescence (FO). 


\section{Discussion}

CPVT is a severe hereditary ion-channel disease characterized by fatal cardiac arrhythmia in response to physical or emotional stress due to missense mutations in RYR2 or CASQ2. Previous studies showed that mutant RYR2 channels led to unwanted $\mathrm{Ca}^{2+}$ release following catecholamine stimulation which could cause DADs, EADs and sudden cardiac death in young patients. (Jiang et al., 2002a; Cerrone et al., 2009).

The molecular mechanisms by which RYR2 mutations trigger arrhythmia are controversially discussed but include weakened inter-domain interactions, modified interaction with accessory proteins, increased phosphorylation of the channel by protein kinases, and altered sensitivity in response to luminal or cytoplasmic $\mathrm{Ca}^{2+}$ concentrations. So far, only $15 \%$ of more than 150 CPVT-causing RYR2 mutations have been studied in HEK293 cells or in a mouse model. The discovery of induced pluripotency generated from somatic cells by ectopic expression of pluripotency-associated transcription factors represented a major breakthrough for medical research (Takahashi and Yamanaka, 2006). Patient-specific hiPSCs function as renewable and unlimited source for CMs and provide the opportunity to investigate the pathophysiology of genetic diseases in vitro.

The aim of this thesis was to establish in vitro disease models using hiPSCs from CPVT patients carrying a heterozygous missense mutation in the $R Y R 2$ gene and to study molecular mechanisms of the disease. First, hiPSC lines were generated from 3 different CPVT patients that are heterozygous for point mutations in the RYR2 gene (A2254V, E4076K) encoding the cardiac RYR2 protein. The generated CPVT-hiPSCs exhibited pluripotent stem cell characteristics demonstrated by pluripotency-related gene and protein expression, in vitro spontaneous differentiation capacity, and in vivo teratoma formation. Furthermore, CPVT-hiPSCs and Ctrl-hiPSCs were directly differentiated into spontaneously beating CMs expressing cardiac-specific genes and proteins. Allele-specific sequencing showed that CMs derived from nearly all CPVT patients have a similar RYR2 mRNA expression of the disease-causing and the healthy allele. Furthermore, CPVT-CMs have similar levels of RYR2 protein expression compared to Ctrl-CMs. Phosphorylation studies showed that RYR2 residues Ser2808 and Ser2814 are highly phosphorylated at basal conditions in CPVT-CMs as well as in Ctrl-CMs. After stimulation with isoprenaline no mutation-specific differences were observed between CPVT-CMs and Ctrl-CMs.

CRISPR/Cas9-triggered genetic engineering was applied for CPVT-hiPSCs using sSODNs and total 807 cell clones were tested after hiPSC transfection and expansion. Two CRISPR/Cas9 targeted clones (RYR2 $2^{\varnothing / \varnothing}$-A3-hiPSCs and RYR2 ${ }^{\varnothing / \varnothing}$-X4-hiPSCs) had homozygous single nucleotide deletions in their genome, which causes a PTC in the RYR2 gene. Another cell line (RYR2 ${ }^{+/ \varnothing}$-T42-hiPSCs) had a heterogeneous $17 \mathrm{bp}$ deletion in the 
CPVT causing allele, which leads to a PTC in the CRISPR binding site. These engineered CRISPR/Cas9 cell lines were differentiated into beating CMs. RYR $2^{\varnothing / \varnothing}$-CMs derived from RYR2 ${ }^{\varnothing / \varnothing}$-A3- and RYR2 ${ }^{\varnothing / \varnothing}$-X4-hiPSCs showed no detectable RYR2 protein expression but normal mRNA expression. However, RYR2 ${ }^{+/ \varnothing}$-CMs derived from RYR2 ${ }^{+/ \varnothing}-$ T42-hiPSCs with heterozygous deletion in the CPVT-causing allele showed normal RYR2 expression but downregulation of the CRISPR/Cas9-targeted allele which normally causes CPVT symptoms. Notably, the analysis of the calcium imaging of $\mathrm{RYR}^{+/ \varnothing}-\mathrm{T} 42-\mathrm{CM}$ showed a reduced $\mathrm{Ca}^{2+}$ leak in comparison to CPVT-CMs. Furthermore, $\mathrm{RYR2}^{+/ \phi}-\mathrm{T} 42-\mathrm{CM}$ resemble a phenotype that is comparable with $\mathrm{Ctrl}-\mathrm{CMs}$ in respect to the $\mathrm{Ca}^{2+}$ spark frequency and FDHM. In contrast, RYR2 ${ }^{\varnothing / \varnothing}$-A3-CMs showed highly significant reduced spark frequency, spark amplitude, FWHM, FDHM and SR $\mathrm{Ca}^{2+}$ leak in comparison to both Ctrl- and CPVTC2CMs. In addition, RYR2 ${ }^{\varnothing / \varnothing}$-A3-CMs have much smaller amplitude and altered shapes of $\mathrm{Ca}^{2+}$ transients when compared to CPVT-CMs and RYR2 ${ }^{+/ \varnothing}-\mathrm{T} 42-\mathrm{CM}$.

\subsection{Successful generation of hiPSCs from CPVT patients}

The generation of hiPSCs by reprogramming somatic cells with defined factors opened the door for disease modeling and medical application of patient-derived engineered stem cells. In this study, SeV was used for reprogramming of CPVT-fibroblasts into hiPSCs. $\mathrm{SeV}$ is a non-segmented negative-strand RNA virus and belongs originally to the paramyxoviridae family. Its RNA replication occurs in the cytoplasm which minimizes the risk for integration into the host genome (Li et al., 2000; Bitzer et al., 2003). SeV was developed as highly efficient transduction vector which enables RNA-based gene delivery of pluripotency-associated factors (Kato et al., 1997; Fusaki et al., 2009b). After viral transduction of somatic cells, SeV vector enables a robust and sustained expression of all four Yamanaka factors OCT4, SOX2, KLF4, and C-MYC for the derivation of transgene-free hiPSCs (Fusaki et al., 2009b).

In the present study, ectopic expression of the four Yamanaka factors in fibroblasts resulted in successful generation of hiPSCs. The reestablishment of pluripotency in a somatic cell is a complicated mechanism which has not been completely understood yet. The most important events during early reprogramming include suppression of somatic cell-specific genes, thereby activating the hESC-specific transcription network, the mesenchymal-to-epithelial transition, and changes from oxidative phosphorylation to a glycolysis-based metabolism (Li et al., 2010; Panopoulos et al., 2012; Zhang et al., 2012a). During the reprogramming process, overexpression of Yamanaka factors also induces a unique epigenetic profile enriched for active chromatin modifications, including trimethylation of histone 3 Lys4 (H3K4me3), H3K36me3, histone acetylation, and 
hypomethylated DNA. These marks are often found within the regions of pluripotencyrelated genes (Apostolou and Hochedlinger, 2013). Furthermore, pluripotent stem cells acquire increased number of bivalent domains marked both with H3K4me3 and H3K27me3 at differentiation-related genes for fast repression or activation of gene expression by removing H3K4me3 or H3K27me3, respectively (Gladych et al., 2015). SOX2 and OCT4 co-occupy promoters of highly expressed pluripotency genes including their own promoters, thus forming an auto-regulatory loop of pluripotency. This auto-regulatory loop enhances the expression of pluripotency-associated transcription factors, such as NANOG, and signaling components to maintain the state of pluripotency of hESCs and hiPSCs (Chen et al., 2008; Kim et al., 2008). NANOG, a homeodomain protein, is an important component for the maintenance of self-renewal in ESCS (Chambers et al., 2003; Mitsui et al., 2003). KFL4 transcription factor shares about half of its target genes with SOX2 and OCT4 (Jiang et al., 2008). In contrast to OCT4, SOX2 and KFL4, C-MYC targets predominately genes involved in the regulation of cellular proliferation, biosynthetic pathways and metabolism, thereby enhancing reprogramming efficiency (Nakagawa et al., 2008; Kim et al., 2010).

Pluripotency-associated transcription factors including OCT4, SOX2 and NANOG are essential to maintain the pluripotency network of ESCs (La Boyer et al., 2005). These factors among others, such as LIN28, FOXD3, GDF3, SSEA4, or TRA-1-60, are also found to be highly expressed in undifferentiated hESCs and their downregulation is linked to differentiated cells (La Boyer et al., 2005; Kim et al., 2008). The generated hiPSCs in the present study showed hESC-like characteristics with regards to their morphology and mRNA expression of pluripotency-related markers, such as OCT4, SOX2, NANOG, LIN28, FOXD3 and GDF3. Furthermore, SOX2, OCT4, NANOG, LIN28, SSEA4 and TRA-1-60 were expressed on protein level, indicating that the ectopic expression of OCT4, SOX2, KLF4, and $C-M Y C$ initiated the activation of endogenous pluripotency-associated genes and proteins (Kim et al., 2008; Fusaki et al., 2009b).

The ability to differentiate into derivatives of all three germ layers is an important characteristic of human pluripotent stem cells (Itskovitz-Eldor et al., 2000). Spontaneous in vitro differentiation of CPVT-hiPSCs showed that differentiated cells of all cell lines expressed markers that are specific for all three germ layers, demonstrating that they are truly pluripotent. However, cell line isCPVTc2.4 from patient c2 failed to express ectodermal markers on mRNA level but expressed $\beta$-III-TUBULIN on protein level.

Teratoma formation assays is defined as the "gold standard" to prove pluripotency and demonstrates the diverse differentiation potential of hiPSCs (Zhang et al., 2012b). In vivo differentiation of CPVT-hiPSCs revealed tissue-specific cells including derivatives of all three germ layers, such as cartilage (mesoderm), neural rosettes (ectoderm) and 
intestinal tissue (endoderm). The hiPSC lines isCPVTc1.1 and isCPVTc2.3 from patients c1 and $c 2$, respectively, formed teratoma containing endodermal and mesodermal cells, but no neural rosettes. However, gene expression analyses showed expression of the ectodermal markers SYN and TH on mRNA level and $\beta$-III-TUBULIN on protein level indicating that these cell lines were able to differentiate into the ectodermal lineage, too. Sequencing of CPVT-hiPSCs from all three patients confirmed the heterogeneous missense mutation (A2254V, E4076K).

To sum up, the generated patient-specific CPVT-hiPSCs have similar characteristics as hESCs and fulfill the criteria of fully reprogrammed hiPSCs. Because of their somatic origin (human fibroblasts), their further use for research purposes does not raise ethical concerns like hESCs.

\subsection{HiPSCs are able to differentiate directly into functional CMs}

Directed cardiac differentiation of hiPSCs was the first step to establish a disease modeling system from CPVT patients and healthy control subjects. Previous studies often used the formation of EBs in medium containing FBS to differentiate hESCs and hiPSCs into $\mathrm{CMs}$ for disease modeling. However, this method was very inefficient and the culture typically composed less than $1 \%$ cardiomyocytes and provided inconsistent results with different human pluripotent stem cell lines (Kehat et al., 2001). Subsequent methodological improvements concentrated first on imitating the embryonic developmental signals that control mesoderm induction such as Nodal/activin, BMP, WNT and FGF pathways following cardiac specification by inhibition of WNT, BMP and TGF $\beta$ signaling (Kattman et al., 2011; Burridge et al., 2012).

In this present study, we tested two monolayer-based cardiac differentiation protocols using small molecule-based approaches with progressive treatment of CHIR99021 and IWP2 for temporal WNT signaling activation and repression, respectively, as previously described (Lian et al., 2012; Burridge et al., 2014). To achieve robust cardiac differentiation, we combined both protocols and generated functional CMs with high efficiency. The first cardiac differentiation protocol for monolayer culture was based on cardiac differentiation medium containing RPMI1640 with B27 supplement. Application of small molecules enabled high efficiency of CM differentiation (Lian et al., 2012; Lian et al., 2013). However, B27 supplement is quite expensive, and contains a complex mixture of various substances from animal origin, and it was originally designed for culturing hippocampal neurons (Brewer et al., 1993). The animal origin could lead to variations in different batch of the B27 supplement which in turn leads to inconsistent CM differentiation. Burridge et al. screened the components of B27 and found that few 
medium ingredients were required for efficient differentiation of hiPSCs into CMs. Along with the treatment of small molecules, the combination of human recombinant albumin, ascorbic acid and basal medium RPMI1640 was essential for robust and efficient CM differentiation. The great advantage of this protocol was the lower prize compared to cardiac differentiation medium supplemented with B27. However, CMs derived by using Burridge's protocol often detached from cell culture dishes, stopped beating spontaneously and showed immature action potentials by patch clamp after longer cultivation periods (60-90 days) (data from W. Li). For this reason, we combined two protocols and switched the medium after 6 days of differentiation again to RPMI 1640 with B27 until further experiments and the previously described problems disappeared. Previous studies, which compared the electrophysiological phenotype of one- and three-month-old hiPSC-derived CMs, reported that CMs following 3 months of in vitro culturing reached a more mature phenotype indicated by higher sodium currents (Stauske et al., unpublished data). Because first symptoms of CPVT manifest during childhood and young adolescence, CMs involved in the CPVT project were cultivated for three months after starting differentiation to increase maturation. After cultivation for 90 days, up to $98 \%$ of the CMs were stained positive for the cardiac marker CTNT. To obtain these pure hiPSC-derived CMs, metabolic selection with lactate instead of glucose was performed one month after starting differentiation. For older cells (>30 days), metabolic selection was less effective and CMs without selection were often overgrown with other neural or fibroblast-like cell types.

For the directed differentiation of CMs, we used Ctrl-hiPSCs and CPVT-hiPSCs generated by STEMCCA and SeV because some hiPSC lines, especially from CPVT patient c2, were not able to differentiate stably or efficiently into CMs. One major disadvantage of the STEMCCA-derived hiPSCs is that the lentivirus is able to integrate its gene fragments randomly into the host genome, thereby causing potential gene disruptions. In this study, no significant differences were observed in hiPSCs generated by using the STEMCCA and $\mathrm{SeV}$ systems in respect to their pluripotency and cardiac differentiation efficiency. Furthermore, we did not observe functional differences between CMs derived from STEMCCA or SeV virus. Some hiPSC lines had variable differentiation efficiencies for the generation of CMs, but these circumstances seem to be random and were not linked to the patient's cell origin or the virus used for reprogramming.

The data of this study showed that hiPSC-derived Ctrl- and CPVT-CMs expressed sarcomeric and gap junction proteins which are essential for cardiac function without differences. Immunocytological staining showed cardiac-specific protein expression in CPVT- and Ctrl-CMs, represented by staining with antibodies against MLC2V, MLC2A, $\alpha-A C T$, and CX43. These data correspond with the data of previous studies that 
differentiate hiPSCs directly into CMs (Burridge et al., 2014). Furthermore, antibody stainings showed organized cross-striations of RYR2, $\alpha-A C T$ and F-ACTIN. CX43 was expressed at cell-to-cell contacts of $\mathrm{CMs}$, indicating the presence of gap junctions which are important for conducting electrical signals between adjacent CMs. Ctrl- and CPVT$\mathrm{CMs}$ showed no differences regarding sarcomeric organization and gap junctions, indicating they are structurally comparable. This is consistent with the clinical findings that patients diagnosed with CPVT have a structurally normal heart (Priori et al., 2002).

Electrophysiological recordings of Ctrl- and CPVT-CMs revealed in respect to the AP shape that our direct cardiac differentiation protocol generates a mixture of the three major cardiac subtypes, named ventricular-, atrial-, and nodal-like CMs (W. Li, unpublished data) with properties similar to hESC-derived CMs (He et al., 2003). By using the differentiation protocol described here, more than $80 \%$ of the CMs showed a ventricular-like phenotype indicated by major expression of the ventricular-specific marker MLC2V and electrophysiological analysis. Around $15 \%$ of the differentiated $\mathrm{CMs}$ were characterized as nodal-like CMs and 5\% of them were atrial-like CMs (data from W. Li).

\subsection{CPVT-CMs as model for studying disease-specific phenotypes in vitro}

CPVT was first described in 1995 and considered a rare syndrome, which is triggered by surge of catecholamines and characterized by polymorphic ventricular arrythmia that usually occurs during physical exercise or emotional stress (Leenhardt et al., 1995). Molecular screenings revealed that $65 \%$ of the patients have mutations in $R Y R 2$. The minor percentages of the patients have mutations in CASQ2, TRDN and JCN. Patients with CPVT a normal 12-lead ECG at rest (Leenhardt et al., 1995). Although ECG abnormalities are diverse during exercise testing, bidirectional VT are recognized in many patients and considered as principle sign of the disease (Baher et al., 2011; Priori and Chen, 2011). RYR2 is an important regulator of $\mathrm{Ca}^{2+}$ homeostasis in CMs and mutations in RYR2 are often associated with hyperactive channels that are prone for unwanted $\mathrm{Ca}^{2+}$ release which may trigger arrhythmias. However, loss-of-function mutations causing hypoactive RYR2 channels with decreased open channel probabilities in response to $\mathrm{Ca}^{2+}$ and caffeine were also reported (Jiang et al., 2007; Zhao et al., 2015). Although the mortality rate of the disease is extremely high (30-35\% by the age of 35 years), therapies are limited. During the last decades, $\beta$-blockers such as metoprolol and bisoprolol were recommended as first line therapy to reduce the frequency of arrhythmias in patients suffering from CPVT. Despite therapy, approximately $30 \%$ of the patients experience at least one episode of life threating arrhythmia leading to syncope or cardiac arrest and will need implantable cardioverter defibrillator (Priori et al., 2002; Cerrone et al., 2012). 
More recently, new drugs, such as flecainide and dantrolene, improved the treatment possibilities for patients who do not tolerate long treatments with high doses of $\beta$ blockers. However, mechanisms by which RYR2 mutations cause arrhythmias are still not fully understood and remain a matter of debate. This limits the development of new drugs for CPVT patients. So far, the proposed mechanisms include weakened interdomain interactions, altered sensitivity to cytosolic or luminal $\mathrm{Ca}^{2+}$ concentrations, decreased binding affinities of interacting proteins, such as FKBP12.6, and enhanced phosphorylation of RYR2 by protein kinases. The development of individual therapies for CPVT patients requires a crucial understanding of the pathophysiological mechanism that is responsible for the occurring syndromes. In this study, patient-specific hiPSCs were generated from different CPVT patients. CMs derived from these hiPSCs were used to investigate the pathophysiological mechanisms of CPVT on molecular level and we hypothesized that different RYR2 mutations have a different molecular cause for the development of CPVT disease.

The usefulness of patient-derived hiPSCs for modelling diseases in vitro has recently been tested for several channelopathies, including among others LQTS type 1 (Moretti et al., 2010), type 2 (Itzhaki et al., 2011a; Matsa et al., 2011; Lahti et al., 2012) and type 3 (Ma et al., 2013) as well as Timothy syndrome (Yazawa et al., 2011) and CPVT (Fatima et al., 2011).

\subsubsection{Pathophysiological comparison of clinical data and hiPSC-derived CPVT-CMs}

The present study established hiPSC models from 6 different CPVT patients and used hiPSCs-derived CMs to understand the underlying molecular mechanism causing CPVT. Disease modeling with hiPSCs from CPVT patients overcomes the impossibility of harvesting adult human CMs from patients and expanding them in culture. Furthermore, diverse hiPSC model systems could facilitate the screening and development of new candidate drugs for the treatment of CPVT on a patient-specific level. Previous disease models studying CPVT were based on in vitro models expressing mutant versions of RYR2 in heterologous cell line systems, lipid bilayers and transgenic knock-out as well as knockin mouse carrying specific RYR2 mutations (Jiang et al., 2002a; Cerrone et al., 2005). These models suggest already that arrhythmias in CPVT are provoked by diastolic $\mathrm{Ca}^{2+}$ leak from the SR, thereby triggering DADs following adrenergic stimulation (Cerrone et al., 2009). But considering the large number of point mutations reported in $R Y R 2$, it is difficult to generalize their different $\mathrm{Ca}^{2+}$ signaling profile due to the different proposed mechanisms causing CPVT symptoms. For this reason, different mouse models, including the Ryr2 mutations R2474S (Lehnart et al., 2008), S2246L (Suetomi et al., 2011), P2328S 
(Goddard et al., 2008) and R4496C (Liu et al., 2006; Fernandez-Velasco et al., 2009) were established. Although mouse models might provide a more powerful tool to elucidate the pathophysiological mechanisms of CPVT compared to heterologous expression systems, they have still limited relevance because of striking physiological differences between the mouse and human heart.

Recent studies focused on patient-specific hiPSC disease models to understand CPVT in the human system. Until now hiPSC models from 10 different CPVT patients were generated including the following missense mutations in the RYR2 gene: S406L (Jung et al., 2012), R420Q (Novak et al., 2015), P2328S (Kujala et al., 2012), E2311D (Di Pasquale et al., 2013), F2483I (Fatima et al., 2011; Zhang et al., 2013), P2328S, T2538R, L4115F, Q4201R (Penttinen et al., 2015) and M4109R (Itzhaki et al., 2012). Another patientspecific hiPSC model had a large deletion in exon 3 of RYR2 (Penttinen et al., 2015).

In this study, we used CMs differentiated from hiPSC models that were generated from 6 different CPVT patients carrying four new RYR2 mutations (R420W, A2254V, E4076K, and $\mathrm{H} 4742 \mathrm{Y}$ ). These mutations are localized in different domains of the tetrameric protein RYR2. The domains of the channel include the cytoplasmic, central and channel region and the largest part is the cytoplasmic region. Structural analysis of porcine RYR2 showed that the cytoplasmic region consists of 9 different domains including four armadillo repeats-containing domains, the amino terminal domain (NTD), the handle domain, the helical domain and the central domain, three intertwined SPRY domains, and two domains designating P1 and P2 domains (Peng et al., 2016). Because RYR2 is highly conserved between mammalian species, the mutation R420W is probably located in the NTD, A2254V is located in the helical domain HD1, E4076K is located in the central domain and $\mathrm{H} 4742 \mathrm{Y}$ is localized in the channel domain. All these mentioned mutations were previously not described with hiPSC models. In contrast to other studies, the mutations R420W and E4076K were modeled with two independent patients to investigate if patient-specific differences in the phenotype exist between two patients carrying the same mutation. Electrophysiological analysis showed that CPVT-CMs carrying the RYR2 mutations R420W, A2254V and E4076K develop different severe types of triggered activity with DADs in response to isoprenaline treatment (W. Li, unpublished data). Furthermore, adrenergic stimulation of fluo 4/AM-loaded CPVT-CMs (R420W, A2254V and E4076K) leads to increased spontaneous $\mathrm{Ca}^{2+}$ release determined by $\mathrm{Ca}^{2+}$ spark analysis using confocal line scanning technique (L. Cyganek, unpublished data). Previous literature describes this hyperactive phenotype as gain-of-function mutation with increased $\mathrm{Ca}^{2+}$ release during diastole generating a depolarizing transient inward current when NCX extrudes the released $\mathrm{Ca}^{2+}$. The $\mathrm{Na}^{+}$inward current then causes DADs which can initiate new action potentials following triggered activity when the threshold is 
reached (Priori and Chen, 2011; Leenhardt et al., 2012). Interestingly, CPVT-CMs carrying the mutation R420W (patients a2 and a3) located in NTD develop less arrhythmias and $\mathrm{Ca}^{2+}$ sparks in comparison to CPVT-CMs carrying the mutation A2254V (patient b1) and E4076K (patients $\mathrm{c} 1$ and c2) which are both located in different domains of RYR2, namely helical domain HD1 and central domain. No significant differences were observed between two patients ( $\mathrm{a} 2$ and $\mathrm{a} 3$, or $\mathrm{c} 1$ and $\mathrm{c} 2$ ) with the same mutation. It could be speculated that the severity of CPVT could be correlated to the location of the mutation in different domains, but the number of patients is very small and the genetic background could be also associated to the severity of the phenotype. When the data of the $\mathrm{Ca}^{2+}$ spark frequency in CPVT-CMs were compared to the clinical data of the recruited patients, it is noticed that isoprenaline-stimulated CPVT-CMs derived from patients b1, c1 and $c 2$ have a more severe phenotype compared to CPVT-CMs derived from the patients a2 and a3. The clinical data support these findings partially. Patient c1 has also a highly severe clinical phenotype and the first CPVT symptoms were already recognized with the age of 10 years. Due to recurrent ventricular arrhythmias, left cardiac sympathetic denervation was performed to minimize the frequency of arrhythmias. In contrast, patient b1 showed no symptoms until diagnosis, but CPVT-CMs showed a severe phenotype in response to isoprenaline treatment in vitro. However, the recruited patients are currently very young in average and the severity of disease progression cannot be predicted at the moment.

The clinical data from patient $d 1$, carrying the mutation $\mathrm{H} 4742 \mathrm{Y}$, is similar when compared to the other recruited CPVT patients. But, we observed a completely different phenotype in the CPVT-CMs from patient $\mathrm{d} 1$ in vitro. The hiPSC-derived CMs were prone to develop EADs in combination with less $\mathrm{Ca}^{2+}$ release when compared to Ctrl-CMs, highlighting that this mutation might induce a loss-of-function. Experimental data of a previous study support this explanation because Ryr2-H4742Y as well as Ryr2-A4860G mutation are both located in the channel region of the receptor (Zhao et al., 2015). Zhao and colleagues observed that $\mathrm{CMs}$ isolated from mice heterozygous for the Ryr2 mutation A4860G exhibited depressed channel activity in vitro characterized by a markedly response to luminal $\mathrm{Ca}^{2+}$ and extremely low open probability in the presence of full agonists, such as $\mathrm{Ca}^{2+}$ and caffeine. Furthermore, prolonged APs with EADs were observed during isoprenaline stimulation. ECG recordings of anesthetized Ryr2-A4860G ${ }^{+/}$mice displayed QRS alternans, premature ventricular complexes and bidirectional VTs in comparison to WT mice without symptoms. HEK293T cells expressing recombinant RYR2A4860G mutation displayed depressed activity and loss of luminal $\mathrm{Ca}^{2+}$ sensitivity (Jiang et al., 2007). 
These results demonstrate that our CPVT-CMs carrying different RYR2 mutations (R420W, A2254V, E4076K, and H4742Y) could recapitulate the phenotype of CPVT disease in vitro. Mutation-specific as well as patient-specific differences were observed regarding electrophysiological and $\mathrm{Ca}^{2+}$ confocal imaging properties. Three $R Y R 2$ mutations (R420W, A2254V, and E4076K) resembling the phenotypes that is described as "gain-of-function" mutation. The mutation $\mathrm{H} 4742 \mathrm{Y}$ resembles the phenotype which is described as "loss-offunction". The clinical data of our patients cannot distinguish between both phenotypes. Nonetheless, phenotypic differences between "gain-of-function" and "loss-of-function" mutations might be important for the choice of a potentially successful therapy with new developed rycals, such as S36 and S107. Furthermore, the location of the mutation in the different domains could be also important for the future therapy of CPVT patients.

\subsubsection{Understanding the mechanism of CPVT disease}

To understand the reason for different phenotypes observed in patient-specific hiPSCderived CPVT-CMs, allele-specific expression (ASE) and protein expression studies were performed. In general, ASE describes that two alleles of the same loci are expressed in a different proportion due to epigenetic modification, cis-acting regulatory variation, and post transcriptional modification. ASE is essential for cellular programming and embryonic development and the diversity of cellular phenotypes. To date, various studies which analyzed genome wide ASE in human, mice and cell lines identified hundreds to thousands of genes that display significant imbalanced expression of both alleles (Delaval and Feil, 2004; Wang et al., 2008; Gregg et al., 2010; Ju et al., 2011; Grundberg et al., 2012).

Distinguishing allelic activity is important for studying abundance of abnormal mRNA products. In the extreme case of ASE, monoallelic expression happens, where only one of both alleles is expressed while the second is completely inactive. However, allele-specific sequencing of the RYR2 gene showed no allelic imbalance in CPVT-CMs derived from all 6 CPVT patients, demonstrating that both alleles were equally expressed in differentiated CPVT-CMs. One sample of patient a3 (R420W) showed a slight increased expression of the CPVT-causing allele (59.7\%), but this imbalanced expression was detected in CPVT-CMs showing a milder phenotype leading to controversial results. Sequencing of genomic DNA of CPVT-CMs showed that both alleles are present in the same ratio. So it could be concluded that the severity of arrhythmias in our CPVT-CMs is not related to the allelespecific transcription of RYR2. The ASE data of mouse studies, which showed no difference between WT and mutated RYR2 mRNA expression, endorse this presumption (Cerrone et al., 2005). To examine that different CPVT phenotypes do not arise from 
different protein expression values, RYR2 protein expression of all 6 CPVT-CMs was compared to Ctrl-CMs by western blot analysis, and altered RYR2 expression was not detected in CPVT-CMs. Previous works studying protein changes caused by different RYR2 mutations also showed no altered RYR2 expression when these CMs were compared to Ctrl-CMs (Jung et al., 2012; Di Pasquale et al., 2013).

\subsubsection{Phosphorylation of Ser2808 and Ser2814 in CPVT-CMs}

The RYR2-channel protein complex is modulated by various posttranslational modifications, including phosphorylation, S-nitrolysation and oxidation which are considered contributing to RYR2 leakiness. RYR2 channels contain several phosphorylation sites which are essential for normal heart function. Studies suggest that RYR2 phosphorylation is increased in heart failure and contributes to increased diastolic $\mathrm{Ca}^{2+}$ leak from the SR, thereby causing atrial and ventricular arrhythmias. Physical exercise increases concentrations of catecholamine that binds and activates $\beta$-adrenergic receptors, which in turn activate adenylate cyclase. The resulting increase of cAMP activates CAMP-dependent PKA which phosphorylates important $\mathrm{Ca}^{2+}$ handling proteins including the voltage-gated L-type $\mathrm{Ca}^{2+}$ channel, RYR2 and PLB. During heart failure in mice, stress situations result in downregulation of PDE4D3 as well as protein phosphatases PP1 and PP2A, which contributes to chronically increased PKA inducing phosphorylation of RYR2. These hyperphoshorylation of RYR2 is assumed to decrease the binding of the stabilizing protein FKBP12.6 resulting in diastolic SR Ca ${ }^{2+}$ leak (Wehrens et al., 2005). However, the molecular events leading to RYR2 hyperphosphorylation in CPVT remain incompletely understood and are highly controversial because different groups reported contradictory findings using similar approaches.

Three phosphorylation sites in RYR2 have been confirmed by ${ }^{32} \mathrm{P}$ incorporation assays and shown to be phosphorylated in vivo. The first discovered one was Ser2808 in human and rodents or Ser2809 in rabbit, respectively, which functions as primary target of PKA (Witcher et al., 1991; Marx et al., 2000). Ser2814 (Ser2815 in rabbit) has been shown as target site of CaMKII and Ser2030 is mainly phosphorylated by PKA (Xiao et al., 2006). Mass spectrometry analysis of the RYR2 phosphorylation domain (aa 2699-2904) has revealed additional putative phosphorylation sits at Ser2810, Ser2811, Ser2797 and Thr2876 (Yuchi et al., 2012).

Marks and colleagues found that elevated phosphorylation of position Ser2808 results in dissociation of the auxiliary protein FKBP12.6 from the RYR2 complex leading to an increase in the channel's sensitivity to $\mathrm{Ca}^{2+}$ (Marx et al., 2000). Further studies showed that increased PKA-induced phosphorylation of RYR2 resulted in diastolic SR $\mathrm{Ca}^{2+}$ leak in 
$\mathrm{CMs}$ which contributes to chronically reduced $\mathrm{SR} \mathrm{Ca}^{2+}$ content and diminished contractility in heart failure (Wehrens et al., 2006; Shan et al., 2010). However, other studies could not support these findings. For example, ouabain-induced arrhythmias did not cause differences in the phosphorylation status of RYR2 at position Ser2808 in the heterologous knock-in mice model Ryr2-R4496C ${ }^{+/-}$compared to wildtype mice. Basal levels of phosphorylation were also not changed in Ryr2-R4496C $\mathrm{C}^{+/-}$mice (Sedej et al., 2010).

In our experiments, two different phospho-specific antibodies (anti-Ser2808, anti-Ser2814) were used to study changes in RYR2 phosphorylation by PKA and CAMKII after isoprenaline application of Ctrl- and CPVT-CMs. It was not possible to analyze the phosphorylation site Ser2030 in CPVT-and Ctrl-CMs due to unspecific binding of p-Ser2030 antibody. Other groups also failed to analyze Ser2030 phosphorylation (Li et al., 2013). It could be also speculated that Ser2030 is not phosphorylated in hiPSCderived $\mathrm{CMs}$ or becomes just phosphorylated in response to other conformational changes in RYR2.

In this study, high baseline phosphorylation of the RYR2 residues Ser2808 and Ser2814 was found in both Ctrl- and CPVT-CMs. However, CMs derived from the 5 CPVT patients (a2, a3, b1, c1 and c2) showed no patient-specific differences of their basal phosphorylation status when they were compared to Ctrl-CMs. After $\beta$-adrenergic stimulation with isoprenaline, phosphorylation of both Ser2808 and Ser2814 was slightly increased in CPVT-CMs and Ctrl-CMs, but mutation- or patient- specific differences were again not observed. The combined treatment of isoprenaline and calyculin $A$ increased the phosphorylation in some cases further, but single treatment with calyculin A resulted in increased phosphorylation of RYR2, too. This observation could be explained by the fact that calyculin A is a distinct inhibitor of PP1 and PP2A (Ishihara et al., 1989). PP1 and PP2A were shown to regulate RYR2 phosphorylation. PP1 appears to be the main phosphatase dephosphorylating Ser2808 and Ser2814. But PP2A may also dephosphorylate RYR2 at the CAMKII site Ser2814 (Xiao et al., 2006; Huke and Bers, 2008). Blocking these phosphatases with calyculin A could increase RYR2 phosphorylation. In general, no significant differences were observed between the both phosphorylation sites Ser2808 and Ser2814. In most experiments, both phosphorylation sites were already highly phosphorylated at basal conditions and the detected increase was not significant. The data from Carter et al. agree with these results because they observed that RYR2, isolated from non-failing sheep hearts, were already phosphorylated to $75 \%$ of maximum at Ser2809, which could explain that significantly increased phosphorylation of Ser2808 would be improbable in hiPSC derived-CMs after the isoprenaline and calyculin A treatments. Other groups compared RYR2 phosphorylation of 
Ser2808 and Ser2814 from rat hearts and reported that Ser2814 (15\%) was significantly lower phosphorylated than Ser2808 (69\%) under basal conditions. After stimulation with isoprenaline, these levels were increased to $60 \%$ and $83 \%$, respectively (Li et al., 2013). It could be argued that $15 \mathrm{~min}$ of isoprenaline stimulation was too short to increase phosphorylation of RYR2. But this is contradicted because the cAMP/PKA response of the $\beta$-adrenergic signaling pathway needs to be activated within seconds to increase the heart beat in a "fight-or-flight" situation (Xiao, 2001). Furthermore, electrophysiological analysis and calcium imaging observed first arrhythmias and $\mathrm{Ca}^{2+}$ leaks already after $15-$ 20 min stimulation with isoprenaline.

Previous studies focusing on the two Ryr2 mutations R176Q and G230C in mice are in line with our data showing that CPVT-linked RYR2 mutations do not alter baseline phosphorylation of RYR2 at the residues Ser2808 and Ser2814 (Chelu et al., 2009; Sedej et al., 2010; Meli et al., 2011; Shan et al., 2012). Other studies observed an elevated phosphorylation after pacing of $\mathrm{CMs}$, but they observed again no differences between wildtype and mutated receptors (Chelu et al., 2009). However, Loaiza and colleagues stimulated mice bearing the heterogeneous Ryr2 mutation V2475F with PKA and reported that phosphorylation of Ser2808 and Ser2030 was increased in comparison to wildtype mice. In addition, these mice displayed adrenergically triggered-increased tachycardias, cytosolic $\mathrm{Ca}^{2+}$ sensitivity and increased activation by luminal $\mathrm{Ca}^{2+}$ (Loaiza et al., 2013).

Taken together, data from this study together with previous results limit the hypothesis that RYR2 hyperphosphorylation is one of the mechanisms causing CPVT symptoms in our CPVT-CMs in response to adrenergic stimulation. Instead, it could be rather suggested that hyperphosphorylation is mutation-specific and causes increased $\mathrm{Ca}^{2+}$ leak if the FKBP12.6 binding site is destroyed with specific RYR2 mutations thereby preventing binding of the stabilizer FKBP12.6. Finally, our data support the hypothesis that phosphorylation of RYR2 is not altered in CPVT patients because we did not observe differences between any hiPSC-derived CMs from healthy subjects and CPVT patients. Phosphorylation of RYR2 seems to be essential for normal channel activity but increased phosphorylation might not be the cause for arrhythmias and $\mathrm{Ca}^{2+}$ leak in CPVT patients, at least in patients carrying the mutations analyzed in this study. More recent studies showed that the ablation of RYR2 phosphorylation at Ser2808 cause worsened $\mathrm{Ca}^{2+}$ mishandling and cardiac dysfunction in triple mutated mice with Ryr2-Ser2808 $\mathrm{A}^{+/-}$ deficient expression of CASQ2 and overexpression of SERCA1 (Liu et al., 2014a). 


\subsection{CRISPR/Cas9 as tool for efficient genome editing in hiPSCs}

The discovery that the bacterial CRISPR/Cas9 defense system can be utilized to generate DSBs in eukaryotic genomes has resulted in a new technology which is able to disrupt, knock-in and correct endogenous genes. Nowadays, CRISPR/Cas9 has rapidly emerged into one of the most popular and efficient techniques for genome editing with successful application in a wide range of biological systems including human cells (Cong et al., 2013; Jinek et al., 2013; Mali et al., 2013).

Custom-engineered CRISPR/Cas9 also holds enormous therapeutic potential for the treatment of genetic disorders by directly correcting disease-causing mutations. The target DNA sequence corresponds to the complementary $20 \mathrm{nt}$ gRNA that is needed to guide CRISPR/Cas9 to the desired location for inducing DSBs. The repair of the broken DNA ends occurs either through NHEJ or HDR. The NHEJ repair mechanism generates indels, which have been utilized to generate random genomic mutations. Alternatively, HDR occurs in the presence of donor DNA plasmids or ssODNs, which contain homologous sequences flanking the DSBs sites to generate site-directed gene fragments leading to targeted gene mutations or corrections.

In this study, a protocol for CRISPR/Cas9-triggered gene targeting was established in CPVT-hiPSCs using sSODNs and a combined customized CRISPR/Cas9 plasmid for transfection of hiPSCs. Using this protocol, we wanted to apply CRISPR/Cas9 simultaneously for NHEJ-mediated deletions causing a reading frame shift in the RYR2 gene as well as for HDR-mediated knock-in to correct the CPVT-causing point mutation in the $R Y R 2$ gene. We hypothesized that the latter mentioned process is able to rescue the CPVT phenotype in CRISPR/Cas9-edited CMs.

To correct the CPVT-causing point mutation by HDR after the induction of DSBs, sSODNs were designed to contain the desired bp located in the center of the ssODNs. High targeting frequencies were reported when the mutation is located in close proximity ( $<20 \mathrm{bp}$ ) to the target site of the nuclease (Chen et al., 2011a; Yang et al., 2013). For the direct measurement of gene disruption at the particular locus in the $R Y R 2$ gene by the CRISPR/Cas9 plasmids designed, a mismatch-specific endonuclease assay was performed first to test the cleavage efficiency of our CRISPR/Cas9 plasmids. We measured $25-27 \%$ cleavage efficiency which was comparable to the positive control provided in the kit. Application of genome editing in sensitive pluripotent stem cells has been reported to be much more difficult compared to the efficient and fast genome editing of robust human tumor cell lines (Fu et al., 2013; Mali et al., 2013). To date, CRISPR-mediated gene knockout has worked efficiency using NHEJ-induced indel mutations in mouse ESCs and zygotes, but precise introduction of point mutations or a sequence fragments directed by 
HDR has remained inefficient in hiPSC systems. Until now, few studies were able to correct missense mutations in hiPSCs successfully using CRISPR/Cas9. These studies include the correction of homozygous missense mutation in the $H B B$ gene causing sickle cell disease and the genetic correction of a point mutation in the JAK3 gene causing severe combined immunodeficiency (Chang et al., 2015; Huang et al., 2015; Li et al., 2016). For transfection, these studies used nucleofection in combination with ssODNs and CRISPR/Cas9 constructs or adenoviral-mediated genome editing. In the present study, ssODNs were also used as source for HDR in combination with predesigned CRISPR/Cas9 plasmids for the gene-editing experiments. After transfection of the designed CRISPR/Cas9 plasmids with ssODNs into CPVT-hiPSCs, low transfection efficiencies were observed with around 5\% using nucleofection. However, the vector had GFP integrated and enabled efficient sorting of $\mathrm{GFP}^{+}$cells. One aggravating factor was the low survival rate of hiPSCs after cell sorting. Furthermore, around 30\% of the growing hiPSC colonies were not able to maintain their pluripotent state and differentiated into other cell types. Despite low transfection efficiency and survival rate, total 807 cell clones were screened after hiPSC transfection, sorting and expansion. In future, the survival of the clones could be improved by culturing sorted hiPSC cells in a more nutrient hiPSC medium, such as the new developed StemMACS iPS-Brew XF medium, which is reported to ensure fast recovery after stressful situations. Perhaps, the viability of dissociated single hiPSCs could be further enhanced if a survival cocktail of different small inhibitors, termed SMC4, would be added to the medium instead of single application of TZV after cell sorting (Valamehr et al., 2012).

After expansion and freezing of the CRISPR/Cas9 targeted clones, PCR and restriction digestion revealed, that $1.4 \%$ of the cell clones integrated ssODNs in their genome by HDR. The small percentage of surviving CRISPR/Cas9-targeted clones, which integrated ssODNs corroborates that HDR works highly inefficient in hiPSCs. To improve the efficiency of CRISPR/Cas9-mediated genome editing, several studies tested small molecules, including RAD51, L-755,507 and SCR7 that were reported to enhance HDR after DSBs (Maruyama et al., 2015; Yu et al., 2015; Song et al., 2016). In some experiments, the $\beta 3$-adrenergic receptor agonist L-755,507 was applied, which has been shown to enhance CRISPR/Cas9-mediated HDR in hiPSCs (Yu et al., 2015). In contrast to the previous study, we did not notice a significant increase of HDR-targeted clones that integrated the SSODNs into their genome.

After PCR amplification and positive restriction digestion with ECoRI, 12 CRISPR/Cas9targeted clones were sequenced, and 11 out of 12 were HDR-targeted clones that had ssODNs integrated. However, additional indels closely located to the CRISPR/Cas9 binding site in the RYR2 gene were found in these 11 clones. This suggests that CRISPR/Cas9 
might cleave the DNA again after integration of the sSODNs. To solve this problem, two additional SSODNs were designed with silent missense mutations to prevent further DNA cleavage after HDR. Although recent studies achieved an increase of HDR efficiency after introduction of CRISPR/Cas9-blocking mutations (Paquet et al., 2016), we could not confirm this result even after insertion of 5 silent mutations. Additional deletions were still observed after introduction of the ssODNs by HDR. This observation fits to other reports describing that Cas9-sgRNA complexes are able to tolerate 1-5 mismatches between the sgRNA and the target sequences, thereby creating off-target cuts in genomic DNA (Fu et al., 2013). The high incidence of cutting already integrated ssODNs displays that the designed CRISPR/Cas9 plasmid has probably a high tolerance to mismatches even though the plasmid was customized generated with minimal off-target activity. In human systems it is particular important to design the sgRNA with high specificity because almost half of the human genome consists of repeated sequences. The off-targeting should be absolutely avoided (Hsu et al., 2013; Kuscu et al., 2014). Nowadays, several online tools and algorithms are available to predict putative nuclease off-targeting sites including: the CRISPR Design Tool (Hsu et al., 2013), ZiFiT targeter (Fu et al., 2013), CasFinder (Aach et al., 2014), and E-CRISP (Heigwer et al., 2014). However, all of these tools have certain limitations because off-target risks and cleavage activity cannot be accurately predicted by any algorithm currently available (Ishida et al., 2015). In this regard, the risk of offtarget mutagenesis is also one of the most important obstacles to the future therapeutic use of genome-specific nucleases.

\subsubsection{Generation of CRISPR/Cas9-edited CMs to study RYR2 function in hiPSCs}

Due to the high incidence of indels after HDR in CRISPR/Cas9- targeted hiPSCs, the designed CRISPR/Cas9 vectors in this study are not suited to correct missense mutations in the RYR2 gene. However, this protocol worked effectively for the introduction of genetic alterations in the $R Y R 2$ gene. After sequencing, three exciting CRISPR/Cas9targeted hiPSC clones were found to have beside the integrated SSODNs homozygous as well as heterozygous deletions in $R Y R 2$. Two hiPSC clones (RYR2 ${ }^{\varnothing / \Phi_{-}}$- 3 - and RYR2 ${ }^{\varnothing / \Phi_{-}}$- 4 hiPSCs) had a homozygous deletion of 1 and 4 bps causing a frame shift with a PTC which could inhibit the translation of the RYR2 protein. The third CRISPR/Cas9-edited clone (RYR2 ${ }^{+/ \varnothing}$-T42-hiPSCs) had a 17 bp deletion in the mutated CPVT allele resulting in a PTC, too. These clones were probably not the only generated ones, because PCR screenings revealed several different hiPSC clones with small and large deletions in the PCR amplified RYR2 gene. 
Several mouse knockout models were reported to analyze the function of RYR2, but human models studying knockout of RYR2 with hiPSC-derived CMs are still not available. Homozygous deletion of Ryr2 causes embryonic lethality in $R y r 2^{-}$mice due to cardiac arrest around day 10. Heterozygous $R y r 2^{+/-}$mice showed alterations in ECG recordings and membrane potential in urinary bladder smooth muscle cells (Hotta et al., 2007).

Another study reduced the amount of Ryr2 with an inducible cardiac-specific Ryr ${ }^{-1-}$ knockout in adult mice and observed brachycardia and intermittent tachycardiac arrhythmias if the levels of Ryr2 were significantly reduced (Bround et al., 2012). These results suggested that Ryr 2 is required to maintain normal heart rhythm. However, the precise mechanism causing arrhythmias in conditional $R y r 2^{\%}$ mice was not further analyzed in the previous study. Because it is commonly known that results from mouse models can often not be translated to human models, we wanted to use hiPSC-derived CMs to study the RYR2 function in homozygous RYR2 knockout models.

In this study, CRISPR/Cas9-edited CMs (RYR2 ${ }^{\varnothing / \varnothing}-\mathrm{A} 3, \mathrm{RYR} 2^{\varnothing / \varnothing}-\mathrm{X} 4$, and RYR2 ${ }^{+/ \varnothing}-\mathrm{T} 42$ ) do not show obvious differences in RYR2 mRNA expression in comparison to their corresponding CPVT-CMs. Notably, western blot analysis and immunofluorescence staining showed no detectable RYR2 protein in RYR2 ${ }^{\varnothing / \varnothing}-A 3$ - and RYR2 ${ }^{\varnothing / \varnothing}-\mathrm{X} 4-\mathrm{CMs}$ in comparison to the original CPVT-CMs. Only the full-length RYR2, not the truncated RYR2, was detected in RYR2 ${ }^{+/ \varnothing}$. T42-CMs. These data suggest that the truncated RYR2 resulted from the frame shift in

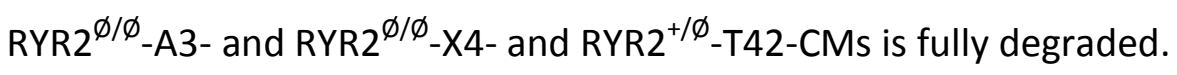

Similar results of full protein degradation caused by CRISPR/Cas9-induced frame shift mutations were also observed by other studies. They simply rescued the phenotype of the genetic skin disorder dominant dystrophic epidermolysis bullosa by knocking out of the mutant allele using specifically induced frame shift mutations (Shinkuma et al., 2016). To investigate the reason for the missing RYR2 protein in RYR2 ${ }^{\varnothing / \phi_{-}}$A3-CMs, we analyzed potential protein degradation in RYR2 ${ }^{\varnothing / \varnothing}-\mathrm{A} 3$ - and $\mathrm{RYR} 2^{+/ \varnothing}-\mathrm{T} 42-\mathrm{CM}$ s by the treatment with the proteasome and calpain inhibitor MG132 and the autophagy inhibitor BafA1 (Pedrozo et al., 2010). But, we were not able to visualize truncated protein versions in RYR2 ${ }^{\varnothing / \varnothing}$-A3and RYR2 $^{+/ \varnothing}$-T42-CMs suggesting a potential nonsense-mediated mRNA decay (NMD) before protein synthesis. NMD is a translation-coupled mRNA surveillance mechanism that helps to maintain the quality of gene expression in eukaryotes. NMD controls newly synthesized mRNAs and degrades those that harbor a PTC, thereby preventing the production of truncated proteins that could result in diseases in humans (Frischmeyer, 1999; Mort et al., 2008). An intron located at least 50-55 bp downstream of a termination codon is sufficient to initiate mammalian NMD. Both homozygous as well as heterozygous $\mathrm{RYR2}^{+/ \varnothing}-\mathrm{T} 42-\mathrm{CMs}$ fulfill these requirements, suggesting that NMD facilitates the degradation of PTC-containing RYR2 mRNA. ASE of mRNA from RYR2 $2^{+/ \Phi_{-T}}$ 2-CMs 
supported this hypothesis because the allele including the PTC is highly decreased (11.4\%) compared to the WT allele (88.6\%). In the future, this result should be repeated with more samples. Nonetheless, the results highlight the generation of RYR2 ${ }^{+/ \varnothing}-\mathrm{T}_{42}-\mathrm{CM}$ with an allele-specific downregulation of the mutated RYR2 mRNA, which would rescue the CPVT phenotype. The potential rescue of the disease phenotype is supported by another study that used RNA interference to downregulate a mutated version of $K C N H 2$ mRNA to rescue the LQT2 phenotype in hiPSC-derived CMs. In that study, electrophysiological analysis of patient-specific LQT2-CMs treated with mutation-specific siRNA showed normalized APDs and $\mathrm{K}^{+}$currents together with concurrent rescue of spontaneous and drug-induced arrhythmias (Matsa et al., 2014). In this study, western blot analysis of RYR2 $^{+/ \varnothing}-$ T42-CMs showed a slight increase of RYR2 expression compared to CPVT-CMs, this might be due to the upregulated mRNA expression of the healthy allele. This result is not in line with the data found in $R y r 2^{+/-}$mice showing the half reduced Ryr2 expression (Hotta et al., 2007). Taken together, the results suggest that the highly reduced expression of the mutated allele might cause a potential healthy phenotype with less or without CPVT-associated symptoms.

The analysis of the $\mathrm{Ca}^{2+}$ sparks in $\mathrm{RYR} 2^{+/ \varnothing}-\mathrm{T} 42-\mathrm{CM}$ s showed that the allele-specific downregulation of the mutated version of RYR2 significantly reduced the $\mathrm{Ca}^{2+}$ sparks under isoprenaline-stimulated conditions. RYR2 ${ }^{+/ \phi}-\mathrm{T}_{42}-\mathrm{CM}$ s have got a phenotype that is comparable with Ctrl-CMs because the spark frequency is on a comparable level, while CPVTc1/c2-CMs have a significantly increased spark frequency in comparison to Ctrl-CMs. Even though it was not possible to correct the missense mutation in the CPVT allele with homologous recombination, we were able to downregulate the mutated allele and verified with this technique that the missense mutation E4076K is probably responsible for CPVT symptoms in patients $\mathrm{c} 1$ and $\mathrm{c} 2$.

Previous studies showed that $\mathrm{CMs}$ isolated from $\mathrm{Ryr}^{+/-}$mice had abnormal $\mathrm{Ca}^{2+}$ release from the SR and reduced contractility at baseline (Zou et al., 2011). This is different from that observed in RYR2 ${ }^{+/ \varnothing}-\mathrm{T} 42-\mathrm{CM}$ in the present study. During pressure overload, which was induced by constriction of transverse aorta, isolated $R y r 2^{+/-}-\mathrm{CMs}$ showed reduced $\mathrm{Ca}^{2+}$ transient amplitudes and increased intracellular $\mathrm{Ca}^{2+}$ concentrations during systole (Zou et al., 2011). However, Ryr2 ${ }^{+/-}$-CMs showed no differences in terms of their morphology, and the heart structure and cardiac contractility in $R y \mathrm{r}^{+/-}$mice were compared to wildtype mice. This indicates that defects of $\mathrm{Ca}^{2+}$ signaling in single cells could be compensated in $\mathrm{Ryr}^{+/-}$mice at organ level.

Because we wondered why RYR $2^{\varnothing / \varnothing}$-A3-CMs were able to beat without RYR2 expression, we speculated that these $\mathrm{CMs}$ have altered expression of other $\mathrm{Ca}^{2+}$ regulatory proteins to compensate the missing RYR2 protein. Studies revealed that hiPSC-derived CMs have 
large differences in their intracellular $\mathrm{Ca}^{2+}$ handling properties compared to adult CMs due to their immature phenotype and their smaller intracellular $\mathrm{Ca}^{2+}$ stores (Satin et al., 2008). For this reason, we speculated that the cardiac IP3Rs might be responsible for compensatory mechanisms involving the contraction of RYR2 ${ }^{\varnothing / \varnothing}-A 3-C M s$ when RYR2 is absent. In adult CMs, IP3Rs seem to regulate only the non-contractile $\mathrm{Ca}^{2+}$ signaling, but abnormal IP3R expression is able to cause arrhythmias (Harzheim et al., 2009). IP3Rs share structural and functional similarities to RYRs, but for their opening IP3 has to bind to the cytosolic part of IP3Rs. Although $\mathrm{Ca}^{2+}$ flux via IP3R is relatively small in comparison to the large whole-cell $\left[\mathrm{Ca}^{2+}\right]_{i}$ transients regulated by RYR2, it is suggested that IP3Rs have an important role in cardiac physiology. IP3Rs are located in the perinuclear region as well as in the nuclear membrane of adult ventricular $\mathrm{CMs}$, but they are expressed in a much smaller density compared to RYRs ( 1:50-1:100) (Moschella and Marks, 1993; Bare et al., 2005). IP3-dependent $\mathrm{Ca}^{2+}$ signaling has been shown to play an important role during the process of cardiac development. In fact, IP3Rs are the first $\mathrm{Ca}^{2+}$ release channels expressed in embryos and the IP3R-mediated $\mathrm{Ca}^{2+}$ release is reported to contribute to spontaneous activity and $\mathrm{Ca}^{2+}$ handling in mouse ESC-CMs and hiPSCs (Kapur and Banach, 2007; Itzhaki et al., 2011b).

In this study, no obvious differences regarding mRNA expression of IP3R1, IP3R2 and CACNA1C were found in RYR2 ${ }^{\varnothing / \varnothing}-A 3-C M s$ in comparison to CPVT-CMs. The protein expression of IP3R, SERCA2A and NCX1 was also not changed in RYR2 ${ }^{\varnothing / \varnothing}-A 3-C M s$. These results suggest that the RYR2 is not necessarily required in CM differentiation from hiPSCs and that IP3-mediated $\mathrm{Ca}^{2+}$ signaling might be important for ECC in RYR2 ${ }^{\varnothing / \varnothing}-\mathrm{A} 3-\mathrm{CMs}$. Similar to the results in RYR2 ${ }^{\varnothing / \varnothing}-\mathrm{A} 3-\mathrm{CMs}$, the expression levels of $\mathrm{Ca}^{2+}$ handling proteins (SERCA2, L-type Ca channel, and NCX) in CMs from $\mathrm{Ryr}^{+/-}$mice were not altered when compared to those from wildtype mice at baseline conditions (Zou et al., 2011). However, they did not analyze IP3R expression. These results implicate that reduced or absent expression of RYR2 might not alter expression of other proteins that are involved in the $\mathrm{Ca}^{2+}$ handling of CMs.

$\mathrm{Ca}^{2+}$ imaging in $\mathrm{RYR} 2^{\varnothing / \varnothing}-\mathrm{A} 3-\mathrm{CM}$ s showed $\mathrm{Ca}^{2+}$ sparks were highly reduced in comparison to CPVT-CMs that reveal high occurrence of sparks at isoprenaline-stimulated conditions. Moreover, amplitudes of $\mathrm{Ca}^{2+}$ transients in RYR2 ${ }^{\varnothing / \varnothing}$-A3-CMs became much smaller. These results suggest that IP3R-mediated $\mathrm{Ca}^{2+}$ release might be implicated and different from RYR2-mediated $\mathrm{Ca}^{2+}$ release even though the expression of IP3R is not changed. The small amplitudes might indicate that $\mathrm{Ca}^{2+}$ cannot be released from the $\mathrm{SR}$, which in turn leads to potentially increased $\left[\mathrm{Ca}^{2+}\right]_{\mathrm{SR}}$ levels in RYR2 ${ }^{\varnothing / \varnothing}-\mathrm{A} 3-\mathrm{CMs}$. 


\subsection{Conclusion and future perspectives}

In conclusion, the findings of this study describe that patient-specific hiPSCs can serve as suitable model for studying RYR2 mutations in the context of CPVT and RYR2-knockout models. CPVT-hiPSCs were successfully differentiated into functional ventricular-like CMs. HiPSC-derived CPVT-CMs display phenotypic features of CPVT symptoms, which partially correlate with different severities of arrhythmias in CPVT patients. Abnormalities of allelespecific mRNA expression or RYR2 protein expression were not found in CPVT-CMs derived from different patients. Furthermore, the data showed that adrenergically stimulated hiPSC-derived CPVT-CMs had no differences in their RYR2 phosphorylation status compared to hiPSC-derived CMs from healthy subjects. Due to these results, this study refutes the hypothesis that stress induces hyperphosphorylation of RYR2 due to missense RYR2 mutations which favor unwanted $\mathrm{Ca}^{2+}$ release from the SR. In future, the underlying molecular mechanism how different $R Y R 2$ mutations favor different severe phenotypes CPVT need to be investigated further. To this end, the cytosolic and luminal $\mathrm{Ca}^{2+}$ sensitivity of RYR2 as well as the SR $\mathrm{Ca}^{2+}$ content should be analyzed by $\mathrm{Ca}^{2+}$ - and caffeine-induced $\mathrm{Ca}^{2+}$ release in CPVT-CMs and compared to Ctrl-CMs. Furthermore, it should be tested if RYR2 mutants have a lowered threshold for SOICR. RYR2 proteins containing different missense mutations from several patients could be isolated to analyze the single channel activity by planar lipid bilayers.

Our studies showed that hiPSC-derived CPVT-CMs are functional and suitable for high throughput screening of new rycals or other small molecules that could restore the RYR2 activity. Furthermore, personalized medicine could predict the response to therapy of individual CPVT patients, which carry different RYR2 mutations causing either "gain-offunction" or "loss-of-function" of RYR2.

Additionally, the CRISPR/Cas9 system was used for genome editing of hiPSCs. CRISPR/Cas9-edited hiPSCs were generated efficiently but it was not possible to generate isogenic controls by ssODNs integration, simultaneously. In general, it would be easier to generate isogenic controls by introducing genetic missense mutations in healthy CtrlhiPSCs. This technique would be faster to generate CRISPR/Cas9-targeted hiPSCs with different RYR2 mutations and it would not be necessary to recruit different CPVT patients from the clinics. Furthermore, the costs and time for reprograming would be reduced. However, cell lines would have the same genetic background and it would be difficult to apply these cells for personalized medicine due to the missing patient-specific background.

In this project, two homozygous CRISPR/Cas9-edited hiPSC lines (RYR2 ${ }^{\varnothing / \varnothing}$-A3- and RYR2 ${ }^{\varnothing / \varnothing}$-X4-hiPSCs) with complete knockout of the RYR2 protein were generated. 
RYR2 ${ }^{\varnothing / \varnothing}$-A3-CMs showed no express of RYR2, have altered $\mathrm{Ca}^{2+}$ transients and are useful to study the $\mathrm{Ca}^{2+}$ regulation in hiPSC-derived $\mathrm{CMs}$, especially during early $\mathrm{CM}$ differentiation. Furthermore, one heterozygous RYR2 ${ }^{+/ \varnothing}-\mathrm{T} 42$-iPSC line with a functional healthy allele of RYR2 was generated. RYR2 ${ }^{+/ \varnothing}-\mathrm{T} 42-\mathrm{CM}$ s display normal RYR2 expression and have highly downregulated expression of the disease-causing allele. These results suggest that RYR2 ${ }^{+/ \varnothing}-\mathrm{T}_{42}-\mathrm{CM}$ s might bear the characteristics of Ctrl-CMs and do not display high incidence of arrhythmias and $\mathrm{Ca}^{2+}$ sparks in response to isoprenaline treatment. The analysis of the $\mathrm{Ca}^{2+}$ sparks, which are significantly reduced in RYR2 ${ }^{+/ \varnothing}-\mathrm{T}_{42}-$ CMs, confirmed this hypothesis and highlights that we were able to rescue CPVT symptoms with CRISPR/Cas9-induced genome editing by knocking out the CPVT-causing allele. In the next step, CRISPR/Cas9-edited CMs should be analyzed by patch-clamp analysis to test if RYR2 ${ }^{+/ \varnothing}-\mathrm{T} 42-\mathrm{CM}$ display a healthy phenotype with reduced arrhythmias that are comparable to Ctrl-CMs. Furthermore, RYR2 ${ }^{\varnothing / \varnothing}-\mathrm{A} 3-\mathrm{CMs}$ should be analyzed by patch-clamp analysis or MEA to test if these CMs display brachycardia, which were observed in Ryr2 ${ }^{-/-}$mice (Bround et al., 2012). 


\section{References}

Aach, J., Mali, P., and Church, G.M. (2014). CasFinder: Flexible algorithm for identifying specific Cas 9 targets in genomes. bioRxiv, 5074.

Apostolou, E., and Hochedlinger, K. (2013). Chromatin dynamics during cellular reprogramming. Nature 502, 462-471.

Ather, S., Respress, J.L., Li, N., and Wehrens, X.H.T. (2013). Alterations in ryanodine receptors and related proteins in heart failure. Biochimica et biophysica acta 1832, 24252431.

Baher, A.A., Uy, M., Xie, F., Garfinkel, A., Qu, Z., and Weiss, J.N. (2011). Bidirectional ventricular tachycardia: ping pong in the His-Purkinje system. Heart rhythm: the official journal of the Heart Rhythm Society 8, 599-605.

Bare, D.J., Kettlun, C.S., Liang, M., Bers, D.M., and Mignery, G.A. (2005). Cardiac type 2 inositol 1,4,5-trisphosphate receptor interaction and and modulation by calcium/calmodulin-dependent protein kinase II. J. Biol. Chem. 280, 15912-15920.

Barrangou, R., Fremaux, C., Deveau, H., Richards, M., Boyaval, P., Moineau, S., Romero, D.A., and Horvath, P. (2007). CRISPR provides acquired resistance against viruses in prokaryotes. Science (New York, N.Y.) 315, 1709-1712.

Bauce, B., Rampazzo, A., Basso, C., Bagattin, A., Daliento, L., Tiso, N., Turrini, P., Thiene, G., Danieli, G.A., and Nava, A. (2002). Screening for ryanodine receptor type 2 mutations in families with effort-induced polymorphic ventricular arrhythmias and sudden death: early diagnosis of asymptomatic carriers. Journal of the American College of Cardiology 40, 341-349.

Bellin, M., Marchetto, M.C., Gage, F.H., and Mummery, C.L. (2012). Induced pluripotent stem cells: the new patient? Nature reviews. Molecular cell biology 13, 713-726.

Ben-Porath, I., Thomson, M.W., Carey, V.J., Ge, R., Bell, G.W., Regev, A., and Weinberg, R.A. (2008). An embryonic stem cell-like gene expression signature in poorly differentiated aggressive human tumors. Nature genetics 40, 499-507.

Bibikova, M., Beumer, K., Trautman, J.K., and Carroll, D. (2003). Enhancing gene targeting with designed zinc finger nucleases. Science (New York, N.Y.) 300, 764.

Bibikova, M., Carroll, D., Segal, D.J., Trautman, J.K., Smith, J., Kim, Y.G., and Chandrasegaran, S. (2001). Stimulation of homologous recombination through targeted cleavage by chimeric nucleases. Molecular and cellular biology 21, 289-297.

Bitzer, M., Armeanu, S., Lauer, U.M., and Neubert, W.J. (2003). Sendai virus vectors as an emerging negative-strand RNA viral vector system. The journal of gene medicine 5, 543553.

Boudoulas, K.D., and Hatzopoulos, A.K. (2009). Cardiac repair and regeneration: the Rubik's cube of cell therapy for heart disease. Disease models \& mechanisms 2, 344-358.

Brennand, K.J., Simone, A., Jou, J., Gelboin-Burkhart, C., Tran, N., Sangar, S., Li, Y., Mu, Y., Chen, G., and $\mathrm{Yu}, \mathrm{D}$., et al. (2011). Modelling schizophrenia using human induced pluripotent stem cells. Nature 473, 221-225. 
Brewer, G.J., Torricelli, JR, Evege, E.K., and Price, P.J. (1993). Optimized survival of hippocampal neurons in B27-supplemented Neurobasal, a new serum-free medium combination. Journal of neuroscience research 35, 567-576.

Briggs, J.A., Sun, J., Shepherd, J., Ovchinnikov, D.A., Chung, T.L., Nayler, S.P., Kao, L.P., Morrow, C.A., Thakar, N.Y., and Soo, S.Y., et al. (2013). Integration-free induced pluripotent stem cells model genetic and neural developmental features of down syndrome etiology. Stem cells (Dayton, Ohio) 31, 467-478.

Bround, M.J., Asghari, P., Wambolt, R.B., Bohunek, L., Smits, C., Philit, M., Kieffer, T.J., Lakatta, E.G., Boheler, K.R., and Moore, E.D., et al. (2012). Cardiac ryanodine receptors control heart rate and rhythmicity in adult mice. Cardiovascular Research 96, 372-380.

Brouns, S.J., Jore, M.M., Lundgren, M., Westra, E.R., Slijkhuis, R.J., Snijders, A.P., Dickman, M.J., Makarova, K.S., Koonin, E.V., and van der Oost, J. (2008). Small CRISPR RNAs guide antiviral defense in prokaryotes. Science (New York, N.Y.) 321, 960-964.

Burridge, P.W., Keller, G., Gold, J.D., and Wu, J.C. (2012). Production of de novo cardiomyocytes: human pluripotent stem cell differentiation and direct reprogramming. Cell stem cell 10, 16-28.

Burridge, P.W., Matsa, E., Shukla, P., Lin, Z.C., Churko, J.M., Ebert, A.D., Lan, F., Diecke, S., Huber, B., and Mordwinkin, N.M., et al. (2014). Chemically defined generation of human cardiomyocytes. Nature methods 11, 855-860.

Campbell, T.J., and Vaughan, W.E.M. (1983). Voltage- and time-dependent depression of maximum rate of depolarisation of guinea-pig ventricular action potentials by two new antiarrhythmic drugs, flecainide and lorcainide. Cardiovascular Research 17, 251-258.

Carvajal-Vergara, X., Sevilla, A., D'Souza, S.L., Ang, Y.S., Schaniel, C., Lee, D.F., Yang, L., Kaplan, A.D., Adler, E.D., and Rozov, R., et al. (2010). Patient-specific induced pluripotent stem-cell-derived models of LEOPARD syndrome. Nature 465, 808-812.

Caspi, O., Huber, I., Gepstein, A., Arbel, G., Maizels, L., Boulos, M., and Gepstein, L. (2013). Modeling of arrhythmogenic right ventricular cardiomyopathy with human induced pluripotent stem cells. Circulation. Cardiovascular genetics 6, 557-568.

Caspi, O., Lesman, A., Basevitch, Y., Gepstein, A., Arbel, G., Habib, I.H.M., Gepstein, L., and Levenberg, S. (2007). Tissue engineering of vascularized cardiac muscle from human embryonic stem cells. Circulation research 100, 263-272.

Cerrone, M., Colombi, B., Santoro, M., Di Barletta, Scelsi, M., Villani, L., Napolitano, C., and Priori, S.G. (2005). Bidirectional ventricular tachycardia and fibrillation elicited in a knock-in mouse model carrier of a mutation in the cardiac ryanodine receptor. Circulation research 96,82 .

Cerrone, M., Cummings, S., Alansari, T., and Priori, S.G. (2012). A clinical approach to inherited arrhythmias. Circulation. Cardiovascular genetics 5, 581-590.

Cerrone, M., Lin, X., Zhang, M., Agullo-Pascual, E., Pfenniger, A., Chkourko Gusky, H., Novelli, V., Kim, C., Tirasawadichai, T., and Judge, D.P., et al. (2014). Missense mutations in plakophilin-2 cause sodium current deficit and associate with a Brugada syndrome phenotype. Circulation 129, 1092-1103.

Cerrone, M., Napolitano, C., and Priori, S.G. (2009). Catecholaminergic polymorphic ventricular tachycardia: A paradigm to understand mechanisms of arrhythmias associated 
to impaired $\mathrm{Ca}^{(2+)}$ regulation. Heart rhythm: the official journal of the Heart Rhythm Society 6, 1652-1659.

Chambers, I., Colby, D., Robertson, M., Nichols, J., Lee, S., Tweedie, S., and Smith, A. (2003). Functional expression cloning of Nanog, a pluripotency sustaining factor in embryonic stem cells. Cell 113, 643-655.

Chandrasekera, P.C., and Pippin, J.J. (2015). The human subject: an integrative animal model for 21(st) century heart failure research. American journal of translational research 7, 1636-1647.

Chang, C.-W., Lai, Y.-S., Westin, E., Khodadadi-Jamayran, A., Pawlik, K.M., Lamb, L.S., JR, Goldman, F.D., and Townes, T.M. (2015). Modeling Human Severe Combined Immunodeficiency and Correction by CRISPR/Cas9-Enhanced Gene Targeting. Cell reports $12,1668-1677$.

Chelu, M.G., Sarma, S., Sood, S., Wang, S., van Oort, R.J., Skapura, D.G., Li, N., Santonastasi, M., Muller, F.U., and Schmitz, W., et al. (2009). Calmodulin kinase IImediated sarcoplasmic reticulum $\mathrm{Ca}+$ leak promotes atrial fibrillation in mice. The Journal of clinical investigation 119, 1940-1951.

Chen, F., Pruett-Miller, S.M., Huang, Y., Gjoka, M., Duda, K., Taunton, J., Collingwood, T.N., Frodin, M., and Davis, G.D. (2011a). High-frequency genome editing using ssDNA oligonucleotides with zinc-finger nucleases. Nature methods 8, 753-755.

Chen, G., Gulbranson, Hou, Z., Bolin, J.M., Ruotti, V., Probasco, M.D., Smuga-Otto, K., Howden, S.E., Diol, N.R., and Propson, N.E., et al. (2011b). Chemically defined conditions for human iPSC derivation and culture. Nature methods 8, 424-429.

Chen, W., Wang, R., Chen, B., Zhong, X., Kong, H., Bai, Y., Zhou, Q., Xie, C., Zhang, J., and Guo, A., et al. (2014). The ryanodine receptor store-sensing gate controls $\mathrm{Ca} 2+$ waves and Ca2+-triggered arrhythmias. Nature medicine 20, 184-192.

Chen, X., Xu, H., Yuan, P., Fang, F., Huss, M., Vega, V.B., Wong, E., Orlov, Y.L., Zhang, W., and Jiang, J., et al. (2008). Integration of external signaling pathways with the core transcriptional network in embryonic stem cells. Cell 133, 1106-1117.

Christian, M., Cermak, T., Doyle, E.L., Schmidt, C., Zhang, F., Hummel, A., Bogdanove, A.J., and Voytas, D.F. (2010). Targeting DNA double-strand breaks with TAL effector nucleases. Genetics 186, 757-761.

Churko, J.M., Burridge, P.W., and Wu, J.C. (2013). Generation of human iPSCs from human peripheral blood mononuclear cells using non-integrative Sendai virus in chemically defined conditions. Methods in molecular biology (Clifton, N.J.) 1036, 81-88.

Chuva de Sousa Lopes, S.M., Hassink, R.J., Feijen, A., van Rooijen, M.A., Doevendans, P.A., Tertoolen, L., La Brutel de Riviere, A., and Mummery, C.L. (2006). Patterning the heart, a template for human cardiomyocyte development. Developmental dynamics: an official publication of the American Association of Anatomists 235, 1994-2002.

Cong, L., Ran, F.A., Cox, D., Lin, S., Barretto, R., Habib, N., Hsu, P.D., Wu, X., Jiang, W., and La Marraffini, et al. (2013). Multiplex genome engineering using CRISPR/Cas systems. Science (New York, N.Y.) 339, 819-823. 
Coumel, P., Fidelle, J., Lucet, V., Attuel, P., and Bouvrain, Y. (1978). Catecholaminergicinduced severe ventricular arrhythmias with Adams-Stokes syndrome in children: report of four cases. Brit. Heart J., 28-37.

Cyganek, L. (2013). Cardiac Progenitor Cells and their Therapeutic Application for Cardiac Repair. J Clin Exp Cardiolog 01.

Delaval, K., and Feil, R. (2004). Epigenetic regulation of mammalian genomic imprinting. Current opinion in genetics \& development 14, 188-195.

Denning, C., Borgdorff, V., Crutchley, J., Firth, K.S.A., George, V., Kalra, S., Kondrashov, A., Hoang, M.D., Mosqueira, D., and Patel, A., et al. (2016). Cardiomyocytes from human pluripotent stem cells: From laboratory curiosity to industrial biomedical platform. Biochimica et biophysica acta 1863, 1728-1748.

DeRosa, B.A., van Baaren, J.M., Dubey, G.K., Lee, J.M., Cuccaro, M.L., Vance, J.M., PericakVance, M.A., and Dykxhoorn, D.M. (2012). Derivation of autism spectrum disorderspecific induced pluripotent stem cells from peripheral blood mononuclear cells. Neuroscience letters 516, 9-14.

Di Pasquale, E., Lodola, F., Miragoli, M., Denegri, M., Avelino-Cruz, J.E., Buonocore, M., Nakahama, H., Portararo, P., Bloise, R., and Napolitano, C., et al. (2013). CaMKII inhibition rectifies arrhythmic phenotype in a patient-specific model of catecholaminergic polymorphic ventricular tachycardia. Cell death \& disease 4, e843.

Diecke, S., Lu, J., Lee, J., Termglinchan, V., Kooreman, N.G., Burridge, P.W., Ebert, A.D., Churko, J.M., Sharma, A., and Kay, M.A., et al. (2015). Novel codon-optimized miniintronic plasmid for efficient, inexpensive, and xeno-free induction of pluripotency. Scientific reports 5, 8081 .

Ding, Q., Regan, S.N., Xia, Y., Oostrom, L.A., Cowan, C.A., and Musunuru, K. (2013). Enhanced efficiency of human pluripotent stem cell genome editing through replacing TALENs with CRISPRs. Cell stem cell 12, 393-394.

Drouin, E., Charpentier, F., Gauthier, C., Laurent, K., and Le Marec, H. (1995). Electrophysiologic characteristics of cells spanning the left ventricular wall of human heart. Evidence for presence of M cells. Journal of the American College of Cardiology 26, 185-192.

Dudek, J., Cheng, I.-F., Balleininger, M., Vaz, F.M., Streckfuss-Bömeke, K., Hübscher, D., Vukotic, M., Wanders, R.J., Rehling, P., and Guan, K. (2013). Cardiolipin deficiency affects respiratory chain function and organization in an induced pluripotent stem cell model of Barth syndrome. Stem Cell Research 11, 806-819.

Eiges, R., Urbach, A., Malcov, M., Frumkin, T., Schwartz, T., Amit, A., Yaron, Y., Eden, A., Yanuka, O., and Benvenisty, N., et al. (2007). Developmental study of fragile $X$ syndrome using human embryonic stem cells derived from preimplantation genetically diagnosed embryos. Cell stem cell 1, 568-577.

Esteban, M.A., Wang, T., Qin, B., Yang, J., Qin, D., Cai, J., Li, W., Weng, Z., Chen, J., and Ni, $S$. , et al. (2010). Vitamin $C$ enhances the generation of mouse and human induced pluripotent stem cells. Cell stem cell 6, 71-79.

Faggioni, M., and Knollmann, B.C. (2012). Calsequestrin 2 and arrhythmias. American journal of physiology. Heart and circulatory physiology 302, H1250-60. 
Fatima, A., Xu, G., Shao, K., Papadopoulos, S., Lehmann, M., Arnáiz-Cot, J.J., Rosa, A.O., Nguemo, F., Matzkies, M., and Dittmann, S., et al. (2011). In vitro Modeling of Ryanodine Receptor 2 Dysfunction Using Human Induced Pluripotent Stem Cells. Cell Physiol Biochem 28, 579-592.

Fernandez-Velasco, M., Rueda, A., Rizzi, N., Benitah, J.-P., Colombi, B., Napolitano, C., Priori, S.G., Richard, S., and Gomez, A.M. (2009). Increased Ca2+ sensitivity of the ryanodine receptor mutant RyR2R4496C underlies catecholaminergic polymorphic ventricular tachycardia. Circulation research 104, 201-9, 12p following 209.

Frischmeyer, P.A. (1999). Nonsense-mediated mRNA decayin health and disease. Human Molecular Genetics 8, 1893-1900.

Frishman, W.H. (2013). beta-Adrenergic blockade in cardiovascular disease. Journal of cardiovascular pharmacology and therapeutics 18, 310-319.

Fu, Y., Foden, J.A., Khayter, C., Maeder, M.L., Reyon, D., Joung, J.K., and Sander, J.D. (2013). High-frequency off-target mutagenesis induced by CRISPR-Cas nucleases in human cells. Nature biotechnology 31, 822-826.

Fusaki, N., Ban, H., Nishiyama, A., Saeki, K., and Hasegawa, M. (2009a). Efficient induction of transgene-free human pluripotent stem cells using a vector based on Sendai virus, an RNA virus that does not integrate into the host genome. Proceedings of the Japan Academy. Series B, Physical and Biological Sciences 85, 348-362.

Fusaki, N., Ban, H., Nishiyama, A., Saeki, K., and Hasegawa, M. (2009b). Efficient induction of transgene-free human pluripotent stem cells using a vector based on Sendai virus, an RNA virus that does not integrate into the host genome. Proceedings of the Japan Academy. Series B, Physical and Biological Sciences 85, 348-362.

George, C.H., Higgs, G.V., and Lai, F.A. (2003). Ryanodine Receptor Mutations Associated With Stress-Induced Ventricular Tachycardia Mediate Increased Calcium Release in Stimulated Cardiomyocytes. Circ Res 93, 531-540.

Gersbach, C.A., and Perez-Pinera, P. (2014). Activating human genes with zinc finger proteins, transcription activator-like effectors and CRISPR/Cas9 for gene therapy and regenerative medicine. Expert opinion on therapeutic targets 18, 835-839.

Gersh, B.J., Maron, B.J., Bonow, R.O., Dearani, J.A., Fifer, M.A., Link, M.S., Naidu, S.S., Nishimura, R.A., Ommen, SR, and Rakowski, H., et al. (2011). 2011 ACCF/AHA Guideline for the Diagnosis and Treatment of Hypertrophic Cardiomyopathy: a report of the American College of Cardiology Foundation/American Heart Association Task Force on Practice Guidelines. Developed in collaboration with the American Association for Thoracic Surgery, American Society of Echocardiography, American Society of Nuclear Cardiology, Heart Failure Society of America, Heart Rhythm Society, Society for Cardiovascular Angiography and Interventions, and Society of Thoracic Surgeons. Journal of the American College of Cardiology 58, 60.

Gherghiceanu, M., Barad, L., Novak, A., Reiter, I., Itskovitz-Eldor, J., Binah, O., and Popescu, L.M. (2011). Cardiomyocytes derived from human embryonic and induced pluripotent stem cells: comparative ultrastructure. Journal of cellular and molecular medicine 15, 2539-2551. 
Gladych, M., Andrzejewska, A., Oleksiewicz, U., and Estecio, M.R.H. (2015). Epigenetic mechanisms of induced pluripotency. Contemporary oncology (Poznan, Poland) 19, A30-8.

Goddard, C.A., Ghais, N.S., Zhang, Y., Williams, A.J., Colledge, W.H., Grace, A.A., and Huang, C.L. (2008). Physiological consequences of the P2328S mutation in the ryanodine receptor (RyR2) gene in genetically modified murine hearts. Acta physiologica (Oxford, England) 194, 123-140.

Gregg, C., Zhang, J., Weissbourd, B., Luo, S., Schroth, G.P., Haig, D., and Dulac, C. (2010). High-resolution analysis of parent-of-origin allelic expression in the mouse brain. Science (New York, N.Y.) 329, 643-648.

Grundberg, E., Small, K.S., Hedman, Å.K., Nica, A.C., Buil, A., Keildson, S., Bell, J.T., Yang, T.P., Meduri, E., and Barrett, A., et al. (2012). Mapping cis- and trans-regulatory effects across multiple tissues in twins. Nature genetics 44, 1084-1089.

Hakamata, Y., Nakai, J., Takeshima, H., and Imoto, K. (1992). Primary structure and distribution of a novel ryanodine receptor/calcium release channel from rabbit brain. FEBS letters 312, 229-235.

Harzheim, D., Movassagh, M., Foo, R.S., Ritter, O., Tashfeen, A., Conway, S.J., Bootman, M.D., and Roderick, H.L. (2009). Increased InsP3Rs in the junctional sarcoplasmic reticulum augment $\mathrm{Ca} 2+$ transients and arrhythmias associated with cardiac hypertrophy. Proceedings of the National Academy of Sciences of the United States of America 106, 11406-11411.

Haurwitz, R.E., Jinek, M., Wiedenheft, B., Zhou, K., and Doudna, J.A. (2010). Sequenceand structure-specific RNA processing by a CRISPR endonuclease. Science (New York, N.Y.) $329,1355-1358$.

He, J.Q., Ma, Y., Lee, Y., Thomson, J.A., and Kamp, T.J. (2003). Human embryonic stem cells develop into multiple types of cardiac myocytes: action potential characterization. Circulation research 93, 32-39.

Heigwer, F., Kerr, G., and Boutros, M. (2014). E-CRISP: fast CRISPR target site identification. Nature methods 11, 122-123.

Heijman, J., Dewenter, M., El-Armouche, A., and Dobrev, D. (2013). Function and regulation of serine/threonine phosphatases in the healthy and diseased heart. Journal of molecular and cellular cardiology 64, 90-98.

Hinson, J.T., Chopra, A., Nafissi, N., Polacheck, W.J., Benson, C.C., Swist, S., Gorham, J., Yang, L., Schafer, S., and Sheng, C.C., et al. (2015). Titin mutations in iPS cells define sarcomere insufficiency as a cause of dilated cardiomyopathy. Science 349, 982-986.

Hockemeyer, D., and Jaenisch, R. (2010). Gene Targeting in Human Pluripotent Cells. Cold Spring Harb Symp Quant Biol 75, 201-209.

Hockemeyer, D., Soldner, F., Beard, C., Gao, Q., Mitalipova, M., DeKelver, R.C., Katibah, G.E., Amora, R., Boydston, E.A., and Zeitler, B., et al. (2009). Efficient targeting of expressed and silent genes in human ESCs and iPSCs using zinc-finger nucleases. Nature biotechnology $27,851-857$. 
Hockemeyer, D., Wang, H., Kiani, S., Lai, C.S., Gao, Q., Cassady, J.P., Cost, G.J., Zhang, L., Santiago, Y., and Miller, J.C., et al. (2011). Genetic engineering of human pluripotent cells using TALE nucleases. Nature biotechnology 29, 731-734.

Hotta, S., Morimura, K., Ohya, S., Muraki, K., Takeshima, H., and Imaizumi, Y. (2007). Ryanodine receptor type 2 deficiency changes excitation-contraction coupling and membrane potential in urinary bladder smooth muscle. The Journal of Physiology 582, 489-506.

Hsu, P.D., Scott, D.A., Weinstein, J.A., Ran, F.A., Konermann, S., Agarwala, V., Li, Y., Fine, E.J., Wu, X., and Shalem, O., et al. (2013). DNA targeting specificity of RNA-guided Cas9 nucleases. Nature biotechnology 31, 827-832.

Huang, X., Wang, Y., Yan, W., Smith, C., Ye, Z., Wang, J., Gao, Y., Mendelsohn, L., and Cheng, L. (2015). Production of Gene-Corrected Adult Beta Globin Protein in Human Erythrocytes Differentiated from Patient iPSCs After Genome Editing of the Sickle Point Mutation. Stem cells (Dayton, Ohio) 33, 1470-1479.

Huangfu, D., Osafune, K., Maehr, R., Guo, W., Eijkelenboom, A., Chen, S., Muhlestein, W., and Melton, D.A. (2008). Induction of pluripotent stem cells from primary human fibroblasts with only Oct4 and Sox2. Nature biotechnology 26, 1269-1275.

Huke, S., and Bers, D.M. (2008). Ryanodine receptor phosphorylation at Serine 2030, 2808 and 2814 in rat cardiomyocytes. Biochemical and biophysical research communications $376,80-85$.

Ikemoto, N., and Yamamoto, T. (2002). Regulation of calcium release by interdomain interaction within ryanodine receptors. Frontiers in bioscience: a journal and virtual library $7,83$.

Inui, M., Saito, A., and Fleischer, S. (1987). Purification of the ryanodine receptor and identity with feet structures of junctional terminal cisternae of sarcoplasmic reticulum from fast skeletal muscle. J. Biol. Chem. 262, 1740-1747.

Ishida, K., Gee, P., and Hotta, A. (2015). Minimizing off-Target Mutagenesis Risks Caused by Programmable Nucleases. International Journal of Molecular Sciences 16, 2475124771.

Ishihara, H., Martin, B.L., Brautigan, D.L., Karaki, H., Ozaki, H., Kato, Y., Fusetani, N., Watabe, S., Hashimoto, K., and Uemura, D., et al. (1989). Calyculin A and okadaic acid: inhibitors of protein phosphatase activity. Biochemical and biophysical research communications 159 , 871-877.

Ishino, Y., Shinagawa, H., Makino, K., Amemura, M., and Nakata, A. (1987). Nucleotide sequence of the iap gene, responsible for alkaline phosphatase isozyme conversion in Escherichia coli, and identification of the gene product. Journal of bacteriology 169, 54295433.

Itskovitz-Eldor, J., Schuldiner, M., Karsenti, D., Eden, A., Yanuka, O., Amit, M., Soreq, H., and Benvenisty, N. (2000). Differentiation of human embryonic stem cells into embryoid bodies compromising the three embryonic germ layers. Molecular medicine (Cambridge, Mass.) 6, 88-95.

Itzhaki, I., Maizels, L., Huber, I., Gepstein, A., Arbel, G., Caspi, O., Miller, L., Belhassen, B., Nof, E., and Glikson, M., et al. (2012). Modeling of catecholaminergic polymorphic 
ventricular tachycardia with patient-specific human-induced pluripotent stem cells. Journal of the American College of Cardiology 60, 990-1000.

Itzhaki, I., Maizels, L., Huber, I., Zwi-Dantsis, L., Caspi, O., Winterstern, A., Feldman, O., Gepstein, A., Arbel, G., and Hammerman, H., et al. (2011a). Modelling the long QT syndrome with induced pluripotent stem cells. Nature 471, 225-229.

Itzhaki, I., Rapoport, S., Huber, I., Mizrahi, I., Zwi-Dantsis, L., Arbel, G., Schiller, J., and Gepstein, L. (2011b). Calcium handling in human induced pluripotent stem cell derived cardiomyocytes. PloS one 6, e18037.

Jasin, M. (1996). Genetic manipulation of genomes with rare-cutting endonucleases. Trends in genetics: TIG 12, 224-228.

Jia, F., Wilson, K.D., Sun, N., Gupta, D.M., Huang, M., Li, Z., Panetta, N.J., Chen, Z.Y., Robbins, R.C., and Kay, M.A., et al. (2010). A nonviral minicircle vector for deriving human iPS cells. Nature methods 7, 197-199.

Jiang, D., Chen, W., Wang, R., Zhang, L., and Chen, S.R.W. (2007). Loss of luminal Ca2+ activation in the cardiac ryanodine receptor is associated with ventricular fibrillation and sudden death. PNAS 104, 18309-18314.

Jiang, D., Wang, R., Xiao, B., Kong, H., Hunt, D.J., Choi, P., Zhang, L., and Chen, S.R.W. (2005). Enhanced store overload-induced $\mathrm{Ca} 2+$ release and channel sensitivity to luminal $\mathrm{Ca} 2+$ activation are common defects of RyR2 mutations linked to ventricular tachycardia and sudden death. Circulation research 97, 1173-1181.

Jiang, D., Xiao, B., Yang, D., Wang, R., Choi, P., Zhang, L., Cheng, H., and Chen, S.R.W. (2004). RyR2 mutations linked to ventricular tachycardia and sudden death reduce the threshold for store-overload-induced Ca2+ release (SOICR). PNAS 101, 13062-13067.

Jiang, D., Xiao, B., Zhang, L., and Chen, S.W. (2002a). Enhanced Basal Activity of a Cardiac $\mathrm{Ca}^{2+}$ Release Channel (Ryanodine Receptor) Mutant Associated With Ventricular Tachycardia and Sudden Death. Circ Res 91, 218-225.

Jiang, D., Xiao, B., Zhang, L., and Chen, SR (2002b). Enhanced basal activity of a cardiac $\mathrm{Ca}^{2+}$ release channel (ryanodine receptor) mutant associated with ventricular tachycardia and sudden death. Circulation research 91, 218-225.

Jiang, J., Chan, Y.-S., Loh, Y.-H., Cai, J., Tong, G.-Q., Lim, C.-A., Robson, P., Zhong, S., and $\mathrm{Ng}, \mathrm{H} . \mathrm{H}$. (2008). A core Klf circuitry regulates self-renewal of embryonic stem cells. Nature cell biology 10, 353-360.

Jinek, M., East, A., Cheng, A., Lin, S., Ma, E., and Doudna, J. (2013). RNA-programmed genome editing in human cells. eLife Sciences 2, e00471.

Ju, Y.S., Kim, J.I., Kim, S., Hong, D., Park, H., Shin, J.Y., Lee, S., Lee, W.C., Yu, S.B., and Park, S.S., et al. (2011). Extensive genomic and transcriptional diversity identified through massively parallel DNA and RNA sequencing of eighteen Korean individuals. Nature genetics $43,745-752$.

Jung, C.B., Moretti, A., Mederos, y.S.M., lop, L., Storch, U., Bellin, M., Dorn, T., Ruppenthal, S., Pfeiffer, S., and Goedel, A., et al. (2012). Dantrolene rescues arrhythmogenic RYR2 defect in a patient-specific stem cell model of catecholaminergic polymorphic ventricular tachycardia. EMBO molecular medicine 4, 180-191. 
Kapur, N., and Banach, K. (2007). Inositol-1,4,5-trisphosphate-mediated spontaneous activity in mouse embryonic stem cell-derived cardiomyocytes. The Journal of Physiology 581, 1113-1127.

Karakikes, I., Stillitano, F., Nonnenmacher, M., Tzimas, C., Sanoudou, D., Termglinchan, V., Kong, C.-W., Rushing, S., Hansen, J., and Ceholski, D., et al. (2015). Correction of human phospholamban R14del mutation associated with cardiomyopathy using targeted nucleases and combination therapy. Nature Communications 6 .

Kato, A., Kiyotani, K., Sakai, Y., Yoshida, T., and Nagai, Y. (1997). The paramyxovirus, Sendai virus, $\mathrm{V}$ protein encodes a luxury function required for viral pathogenesis. The EMBO Journal 16, 578-587.

Kattman, S.J., Witty, A.D., Gagliardi, M., Dubois, N.C., Niapour, M., Hotta, A., Ellis, J., and Keller, G. (2011). Stage-specific optimization of activin/nodal and BMP signaling promotes cardiac differentiation of mouse and human pluripotent stem cell lines. Cell stem cell 8 , 228-240.

Kehat, I., Kenyagin-Karsenti, D., Snir, M., Segev, H., Amit, M., Gepstein, A., Livne, E., Binah, O., Itskovitz-Eldor, J., and Gepstein, L. (2001). Human embryonic stem cells can differentiate into myocytes with structural and functional properties of cardiomyocytes. The Journal of clinical investigation 108, 407-414.

Kim, B.-Y., Jeong, S., Lee, S.-Y., Lee, S.M., Gweon, E.J., Ahn, H., Kim, J., and Chung, S.-K. (2016). Concurrent progress of reprogramming and gene correction to overcome therapeutic limitation of mutant ALK2-iPSC. Experimental \& molecular medicine 48, e237.

Kim, D., Kim, C.H., Moon, J.I., Chung, Y.G., Chang, M.Y., Han, B.S., Ko, S., Yang, E., Cha, K.Y., and Lanza, R., et al. (2009). Generation of human induced pluripotent stem cells by direct delivery of reprogramming proteins. Cell stem cell 4, 472-476.

Kim, J., Chu, J., Shen, X., Wang, J., and Orkin, S.H. (2008). An extended transcriptional network for pluripotency of embryonic stem cells. Cell 132, 1049-1061.

Kim, J., Woo, A.J., Chu, J., Snow, J.W., Fujiwara, Y., Kim, C.G., Cantor, A.B., and Orkin, S.H. (2010). A Myc network accounts for similarities between embryonic stem and cancer cell transcription programs. Cell 143, 313-324.

Kim, Y.G., Cha, J., and Chandrasegaran, S. (1996). Hybrid restriction enzymes: zinc finger fusions to Fok I cleavage domain. Proceedings of the National Academy of Sciences of the United States of America 93, 1156-1160.

Kujala, K., Paavola, J., Lahti, A., Larsson, K., Pekkanen-Mattila, M., Viitasalo, M., Lahtinen, A.M., Toivonen, L., Kontula, K., and Swan, H., et al. (2012). Cell model of catecholaminergic polymorphic ventricular tachycardia reveals early and delayed afterdepolarizations. PloS one 7, e44660.

Kuscu, C., Arslan, S., Singh, R., Thorpe, J., and Adli, M. (2014). Genome-wide analysis reveals characteristics of off-target sites bound by the Cas 9 endonuclease. Nature biotechnology 32, 677-683.

Kuttler, F., and Mai, S. (2006). c-Myc, Genomic Instability and Disease. Genome dynamics 1, 171-190.

Kvam, D.C., Banitt, E.H., and Schmid, JR (1984). Antiarrhythmic and electrophysiologic actions of flecainide in animal models. The American journal of cardiology 53, 22-25. 
La Boyer, Lee, T.I., Cole, M.F., Johnstone, S.E., Levine, S.S., Zucker, J.P., Guenther, M.G., Kumar, R.M., Murray, H.L., and Jenner, R.G., et al. (2005). Core transcriptional regulatory circuitry in human embryonic stem cells. Cell 122, 947-956.

Lahti, A.L., Kujala, V.J., Chapman, H., Koivisto, A.P., Pekkanen-Mattila, M., Kerkela, E., Hyttinen, J., Kontula, K., Swan, H., and Conklin, B.R., et al. (2012). Model for long QT syndrome type 2 using human iPS cells demonstrates arrhythmogenic characteristics in cell culture. Disease models \& mechanisms 5, 220-230.

Lai, F.A., Erickson, H.P., Rousseau, E., Liu, Q.Y., and Meissner, G. (1988). Purification and reconstitution of the calcium release channel from skeletal muscle. Nature 331, 315-319.

Laitinen, P.J., Swan, H., and Kontula, K. (2003). Molecular genetics of exercise-induced polymorphic ventricular tachycardia: identification of three novel cardiac ryanodine receptor mutations and two common calsequestrin 2 amino-acid polymorphisms. European Journal of Human Genetics 11, 888-891.

Lan, F., Lee, A.S., Liang, P., Sanchez-Freire, V., Nguyen, P.K., Wang, L., Han, L., Yen, M., Wang, Y., and Sun, N., et al. (2013). Abnormal Calcium Handling Properties Underlie Familial Hypertrophic Cardiomyopathy Pathology in Patient-Specific Induced Pluripotent Stem Cells. Cell stem cell 12, 101-113.

Lanner, J.T., Georgiou, D.K., Joshi, A.D., and Hamilton, S.L. (2010). Ryanodine receptors: structure, expression, molecular details, and function in calcium release. Cold Spring Harbor perspectives in biology 2, a003996.

Larson, M.H., La Gilbert, Wang, X., Lim, W.A., Weissman, J.S., and Qi, L.S. (2013). CRISPR interference (CRISPRi) for sequence-specific control of gene expression. Nature protocols 8, 2180-2196.

Lee, A.S., Tang, C., Cao, F., Xie, X., van der Bogt, K., Hwang, A., Connolly, A.J., Robbins, R.C., and Wu, J.C. (2009). Effects of cell number on teratoma formation by human embryonic stem cells. Cell cycle (Georgetown, Tex.) 8, 2608-2612.

Leenhardt, A., Denjoy, I., and Guicheney, P. (2012). Catecholaminergic Polymorphic Ventricular Tachycardia. Circ Arrhythm Electrophysiol 5, 1044-1052.

Leenhardt, A., Lucet, V., Denjoy, I., Grau, F., Ngoc, D.D., and Coumel, P. (1995). Catecholaminergic Polymorphic Ventricular Tachycardia in Children. Circulation 91, 15121519.

Lehnart, S.E., Mongillo, M., Bellinger, A., Lindegger, N., Chen, B.X., Hsueh, W., Reiken, S., Wronska, A., Drew, L.J., and Ward, C.W., et al. (2008). Leaky Ca ${ }^{2+}$ release channel/ryanodine receptor 2 causes seizures and sudden cardiac death in mice. The Journal of clinical investigation 118, 2230-2245.

Li, C., Ding, L., Sun, C.-W., Wu, L.-C., Zhou, D., Pawlik, K.M., Khodadadi-Jamayran, A., Westin, E., Goldman, F.D., and Townes, T.M. (2016). Novel HDAd/EBV Reprogramming Vector and Highly Efficient Ad/CRISPR-Cas Sickle Cell Disease Gene Correction. Scientific reports 6, 30422 .

Li, H.O., Zhu, Y.F., Asakawa, M., Kuma, H., Hirata, T., Ueda, Y., Lee, Y.S., Fukumura, M., lida, A., and Kato, A., et al. (2000). A cytoplasmic RNA vector derived from nontransmissible Sendai virus with efficient gene transfer and expression. Journal of virology 74, 6564-6569. 
Li, J., Imtiaz, M.S., Beard, N.A., Dulhunty, A.F., Thorne, R., vanHelden, D.F., and Laver, D.R. (2013). $\beta$-Adrenergic stimulation increases RyR2 activity via intracellular $\mathrm{Ca} 2+$ and $\mathrm{Mg} 2+$ regulation. PloS one 8 , e58334.

Li, R., Liang, J., Ni, S., Zhou, T., Qing, X., Li, H., He, W., Chen, J., Li, F., and Zhuang, Q., et al. (2010). A mesenchymal-to-epithelial transition initiates and is required for the nuclear reprogramming of mouse fibroblasts. Cell stem cell 7, 51-63.

Lian, X., Hsiao, C., Wilson, G., Zhu, K., Hazeltine, L.B., Azarin, S.M., Raval, K.K., Zhang, J., Kamp, T.J., and Palecek, S.P. (2012). Robust cardiomyocyte differentiation from human pluripotent stem cells via temporal modulation of canonical Wnt signaling. Proceedings of the National Academy of Sciences of the United States of America 109, 57.

Lian, X., Zhang, J., Azarin, S.M., Zhu, K., Hazeltine, L.B., Bao, X., Hsiao, C., Kamp, T.J., and Palecek, S.P. (2013). Directed cardiomyocyte differentiation from human pluripotent stem cells by modulating $W n t / \beta$-catenin signaling under fully defined conditions. Nature protocols $8,162-175$.

Liang, P., Lan, F., Lee, A.S., Gong, T., Sanchez-Freire, V., Wang, Y., Diecke, S., Sallam, K., Knowles, J.W., and Wang, P.J., et al. (2013). Drug screening using a library of human induced pluripotent stem cell-derived cardiomyocytes reveals disease-specific patterns of cardiotoxicity. Circulation 127, 1677-1691.

Lieu, D.K., Fu, J.-D., Chiamvimonvat, N., Tung, K.C., McNerney, G.P., Huser, T., Keller, G., Kong, C.-W., and Li, R.A. (2013). Mechanism-based facilitated maturation of human pluripotent stem cell-derived cardiomyocytes. Circulation. Arrhythmia and electrophysiology 6, 191-201.

Lieu, D.K., Liu, J., Siu, C.W., McNerney, G.P., Tse, H.F., Abu-Khalil, A., Huser, T., and Li, R.A. (2009). Absence of transverse tubules contributes to non-uniform $\mathrm{Ca}^{(2+)}$ wavefronts in mouse and human embryonic stem cell-derived cardiomyocytes. Stem cells and development 18, 1493-1500.

Liu, B., Ho, H.T., Velez-Cortes, F., Lou, Q., Valdivia, C.R., Knollmann, B.C., Valdivia, H.H., and Gyorke, S. (2014a). Genetic ablation of ryanodine receptor 2 phosphorylation at Ser2808 aggravates $\mathrm{Ca}^{(2+)}$-dependent cardiomyopathy by exacerbating diastolic $\mathrm{Ca}^{2+}$ release. The Journal of Physiology 592, 1957-1973.

Liu, H., Chen, Y., Niu, Y., Zhang, K., Kang, Y., Ge, W., Liu, X., Zhao, E., Wang, C., and Lin, S., et al. (2014b). TALEN-mediated Gene Mutagenesis in Rhesus and Cynomolgus Monkeys. Cell stem cell 14, 323-328.

Liu, N., Colombi, B., Memmi, M., Zissimopoulos, S., Rizzi, N., Negri, S., Imbriani, M., Napolitano, C., Lai, F.A., and Priori, S.G. (2006). Arrhythmogenesis in catecholaminergic polymorphic ventricular tachycardia: insights from a RyR2 R4496C knock-in mouse model. Circulation research 99, 292-298.

Liu, N., Denegri, M., Ruan, Y., Avelino-Cruz, J.E., Perissi, A., Negri, S., Napolitano, C., Coetzee, W.A., Boyden, P.A., and Priori, S.G. (2011). Short communication: flecainide exerts an antiarrhythmic effect in a mouse model of catecholaminergic polymorphic ventricular tachycardia by increasing the threshold for triggered activity. Circulation research 109, 291-295. 
Loaiza, R., Benkusky, N.A., Powers, P.P., Hacker, T., Noujaim, S., Ackerman, M.J., Jalife, J., and Valdivia, H.H. (2013). Heterogeneity of Ryanodine Receptor Dysfunction in a Mouse Model Of Catecholaminergic Polymorphic Ventricular Tachycardia. Circulation research 112, 298-308.

Lundy, S.D., Zhu, W.-Z., Regnier, M., and Laflamme, M.A. (2013). Structural and functional maturation of cardiomyocytes derived from human pluripotent stem cells. Stem cells and development 22, 1991-2002.

Ma, D., Wei, H., Zhao, Y., Lu, J., Li, G., Sahib, N.B., Tan, T.H., Wong, K.Y., Shim, W., and Wong, P., et al. (2013). Modeling type 3 long QT syndrome with cardiomyocytes derived from patient-specific induced pluripotent stem cells. International journal of cardiology 168, 5277-5286.

Makarova, K.S., Haft, D.H., Barrangou, R., Brouns, S.J., Charpentier, E., Horvath, P., Moineau, S., Mojica, F.J., Wolf, Y.I., and Yakunin, A.F., et al. (2011). Evolution and classification of the CRISPR-Cas systems. Nature reviews. Microbiology 9, 467-477.

Mali, P., Chou, B.K., Yen, J., Ye, Z., Zou, J., Dowey, S., Brodsky, R.A., Ohm, J.E., Yu, W., and Baylin, S.B., et al. (2010). Butyrate greatly enhances derivation of human induced pluripotent stem cells by promoting epigenetic remodeling and the expression of pluripotency-associated genes. Stem cells (Dayton, Ohio) 28, 713-720.

Mali, P., Yang, L., Esvelt, K.M., Aach, J., Guell, M., DiCarlo, J.E., Norville, J.E., and Church, G.M. (2013). RNA-guided human genome engineering via Cas9. Science (New York, N.Y.) 339, 823-826.

Maruyama, T., Dougan, S.K., Truttmann, M.C., Bilate, A.M., Ingram, J.R., and Ploegh, H.L. (2015). Increasing the efficiency of precise genome editing with CRISPR-Cas9 by inhibition of nonhomologous end joining. Nature biotechnology 33, 538-542.

Marx, S.O., and Marks, A.R. (2013). Dysfunctional ryanodine receptors in the heart: new insights into complex cardiovascular diseases. Journal of molecular and cellular cardiology $58,225-231$.

Marx, S.O., Reiken, S., Hisamatsu, Y., Gaburjakova, M., Gaburjakova, J., Yang, Y.M., Rosemblit, N., and Marks, A.R. (2001). Phosphorylation-dependent regulation of ryanodine receptors: a novel role for leucine/isoleucine zippers. The Journal of cell biology 153, 699-708.

Marx, S.O., Reiken, S., Hisamatsu, Y., Jayaraman, T., Burkhoff, D., Rosemblit, N., and Marks, A.R. (2000). PKA phosphorylation dissociates FKBP12.6 from the calcium release channel (ryanodine receptor): defective regulation in failing hearts. Cell 101, 365-376.

Matsa, E., Dixon, J.E., Medway, C., Georgiou, O., Patel, M.J., Morgan, K., Kemp, P.J., Staniforth, A., Mellor, I., and Denning, C. (2014). Allele-specific RNA interference rescues the long-QT syndrome phenotype in human-induced pluripotency stem cell cardiomyocytes. European Heart Journal 35, 1078-1087.

Matsa, E., Rajamohan, D., Dick, E., Young, L., Mellor, I., Staniforth, A., and Denning, C. (2011). Drug evaluation in cardiomyocytes derived from human induced pluripotent stem cells carrying a long QT syndrome type 2 mutation. European Heart Journal 32, 952-962.

Meissner, G. (1986). Ryanodine activation and inhibition of the Ca2+ release channel of sarcoplasmic reticulum. J. Biol. Chem. 261, 6300-6306. 
Meli, A.C., Refaat, M.M., Dura, M., Reiken, S., Wronska, A., Wojciak, J., Carroll, J., Scheinman, M.M., and Marks, A.R. (2011). A Novel Ryanodine Receptor Mutation Linked to Sudden Death Increases Sensitivity to Cytosolic Calcium. Circ Res 109, 281-290.

Mitsui, K., Tokuzawa, Y., Itoh, H., Segawa, K., Murakami, M., Takahashi, K., Maruyama, M., Maeda, M., and Yamanaka, S. (2003). The homeoprotein Nanog is required for maintenance of pluripotency in mouse epiblast and ES cells. Cell 113, 631-642.

Mohamed, B.A., Neef, S., Lbik, D., Khadjeh, S., Sossalla, S., Lehnart, S., Hasenfuss, G., and Toischer, K. (2015). Abstract 14206: The Ryanodine Receptor Calcium Leak Does Not Contribute to the Development of Heart Failure. Circulation 132, A14206-A14206.

Moore, F.E., Reyon, D., Sander, J.D., Martinez, S.A., Blackburn, J.S., Khayter, C., Ramirez, C.L., Joung, J.K., and Langenau, D.M. (2012). Improved somatic mutagenesis in zebrafish using transcription activator-like effector nucleases (TALENs). PloS one 7, e37877.

Moretti, A., Bellin, M., Welling, A., Jung, C.B., Lam, J.T., Bott-Flugel, L., Dorn, T., Goedel, A., Hohnke, C., and Hofmann, F., et al. (2010). Patient-specific induced pluripotent stemcell models for long-QT syndrome. The New England journal of medicine 363, 1397-1409.

Mort, M., Ivanov, D., Cooper, D.N., and Chuzhanova, N.A. (2008). A meta-analysis of nonsense mutations causing human genetic disease. Human mutation 29, 1037-1047.

Moschella, M.C., and Marks, A.R. (1993). Inositol 1,4,5-trisphosphate receptor expression in cardiac myocytes. The Journal of cell biology 120, 1137-1146.

Munos, B. (2009). Lessons from 60 years of pharmaceutical innovation. Nature reviews. Drug discovery 8, 959-968.

Nakagawa, M., Koyanagi, M., Tanabe, K., Takahashi, K., Ichisaka, T., Aoi, T., Okita, K., Mochiduki, Y., Takizawa, N., and Yamanaka, S. (2008). Generation of induced pluripotent stem cells without Myc from mouse and human fibroblasts. Nature biotechnology 26, 101-106.

Nakai, J., Imagawa, T., Hakamat, Y., Shigekawa, M., Takeshima, H., and Numa, S. (1990). Primary structure and functional expression from cDNA of the cardiac ryanodine receptor/calcium release channel. FEBS letters 271, 169-177.

Nihongaki, Y., Yamamoto, S., Kawano, F., Suzuki, H., and Sato, M. (2015). CRISPR-Cas9based photoactivatable transcription system. Chemistry \& biology 22, 169-174.

Novak, A., Barad, L., Lorber, A., Gherghiceanu, M., Reiter, I., Eisen, B., Eldor, L., ItskovitzEldor, J., Eldar, M., and Arad, M., et al. (2015). Functional abnormalities in iPSC-derived cardiomyocytes generated from CPVT1 and CPVT2 patients carrying ryanodine or calsequestrin mutations. Journal of cellular and molecular medicine 19, 2006-2018.

Nyegaard, M., Overgaard, M.T., Sondergaard, M.T., Vranas, M., Behr, E.R., Hildebrandt, L.L., Lund, J., Hedley, P.L., Camm, A.J., and Wettrell, G., et al. (2012). Mutations in calmodulin cause ventricular tachycardia and sudden cardiac death. American journal of human genetics 91, 703-712.

O'Connor, M.D., Kardel, M.D., Iosfina, I., Youssef, D., Lu, M., Li, M.M., Vercauteren, S., Nagy, A., and Eaves, C.J. (2008). Alkaline phosphatase-positive colony formation is a sensitive, specific, and quantitative indicator of undifferentiated human embryonic stem cells. Stem cells (Dayton, Ohio) 26, 1109-1116. 
Okita, K., Ichisaka, T., and Yamanaka, S. (2007). Generation of germline-competent induced pluripotent stem cells. Nature 448, 313-317.

Paavola, J., Viitasalo, M., Laitinen-Forsblom, P.J., Pasternack, M., Swan, H., Tikkanen, I., Toivonen, L., Kontula, K., and Laine, M. (2007). Mutant ryanodine receptors in catecholaminergic polymorphic ventricular tachycardia generate delayed afterdepolarizations due to increased propensity to Ca2+ waves. European Heart Journal $28,1135-1142$.

Paige, S.L., Osugi, T., Afanasiev, O.K., Pabon, L., Reinecke, H., and Murry, C.E. (2010). Endogenous $\mathrm{Wnt} /$ beta-catenin signaling is required for cardiac differentiation in human embryonic stem cells. PloS one 5, e11134.

Panopoulos, A.D., Yanes, O., Ruiz, S., Kida, Y.S., Diep, D., Tautenhahn, R., Herrerias, A., Batchelder, E.M., Plongthongkum, N., and Lutz, M., et al. (2012). The metabolome of induced pluripotent stem cells reveals metabolic changes occurring in somatic cell reprogramming. Cell research 22, 168-177.

Paquet, D., Kwart, D., Chen, A., Sproul, A., Jacob, S., Teo, S., Olsen, K.M., Gregg, A., Noggle, S., and Tessier-Lavigne, M. (2016). Efficient introduction of specific homozygous and heterozygous mutations using CRISPR/Cas9. Nature 533, 125-129.

Pearl, J.I., Lee, A.S., Leveson-Gower, D.B., Sun, N., Ghosh, Z., Lan, F., Ransohoff, J., Negrin, R.S., Davis, M.M., and Wu, J.C. (2011). Short-term immunosuppression promotes engraftment of embryonic and induced pluripotent stem cells. Cell stem cell 8, 309-317.

Pedrozo, Z., Sanchez, G., Torrealba, N., Valenzuela, R., Fernandez, C., Hidalgo, C., Lavandero, S., and Donoso, P. (2010). Calpains and proteasomes mediate degradation of ryanodine receptors in a model of cardiac ischemic reperfusion. Biochimica et biophysica acta $1802,356-362$.

Peng, W., Shen, H., Wu, J., Guo, W., Pan, X., Wang, R., Chen, S.R.W., and Yan, N. (2016). Structural basis for the gating mechanism of the type 2 ryanodine receptor RyR2. Science (New York, N.Y.).

Penttinen, K., Swan, H., Vanninen, S., Paavola, J., Lahtinen, A.M., Kontula, K., and AaltoSetala, K. (2015). Antiarrhythmic Effects of Dantrolene in Patients with Catecholaminergic Polymorphic Ventricular Tachycardia and Replication of the Responses Using iPSC Models. PloS one 10, e0125366.

Postma, A.V., Denjoy, I., Kamblock, J., Alders, M., Lupoglazoff, J.-M., Vaksmann, G., Dubosq-Bidot, L., Sebillon, P., Mannens, M M A M, and Guicheney, P., et al. (2005). Catecholaminergic polymorphic ventricular tachycardia: RYR2 mutations, bradycardia, and follow up of the patients. J Med Genet 42, 863-870.

Priori, S.G., and Chen, S.W. (2011). Inherited Dysfunction of Sarcoplasmic Reticulum Ca2+ Handling and Arrhythmogenesis. Circ Res 108, 871-883.

Priori, S.G., and Napolitano, C. (2005). Cardiac and skeletal muscle disorders caused by mutations in the intracellular Ca2+ release channels. Journal of Clinical Investigation 115, 2033-2038.

Priori, S.G., Napolitano, C., Memmi, M., Colombi, B., Drago, F., Gasparini, M., DeSimone, L., Coltorti, F., Bloise, R., and Keegan, R., et al. (2002). Clinical and Molecular 
Characterization of Patients With Catecholaminergic Polymorphic Ventricular Tachycardia. Circulation 106, 69-74.

Ramos, E., and O'Leary, M.E. (2004). State-dependent trapping of flecainide in the cardiac sodium channel. The Journal of Physiology 560, 37-49.

Ran, F.A., Hsu, P.D., Lin, C.Y., Gootenberg, J.S., Konermann, S., Trevino, A., Scott, D.A., Inoue, A., Matoba, S., and Zhang, Y., et al. (2013). Double nicking by RNA-guided CRISPR Cas9 for enhanced genome editing specificity. Cell 154, 1380-1389.

Ren, Y., Lee, M.Y., Schliffke, S., Paavola, J., Amos, P.J., Ge, X., Ye, M., Zhu, S., Senyei, G., and Lum, L., et al. (2011). Small molecule Wnt inhibitors enhance the efficiency of BMP-4directed cardiac differentiation of human pluripotent stem cells. Journal of molecular and cellular cardiology 51, 280-287.

Rodin, S., Domogatskaya, A., Strom, S., Hansson, E.M., Chien, K.R., Inzunza, J., Hovatta, O., and Tryggvason, K. (2010). Long-term self-renewal of human pluripotent stem cells on human recombinant laminin-511. Nature biotechnology 28, 611-615.

Rodriguez, P., Bhogal, M.S., and Colyer, J. (2003). Stoichiometric phosphorylation of cardiac ryanodine receptor on serine- 2809 by calmodulin-dependent kinase II and protein kinase A. J. Biol. Chem. 278.

Rooryck, C., Kyndt, F., Bozon, D., Roux-Buisson, N., Sacher, F., Probst, V., and Thambo, J.B. (2015). New Family With Catecholaminergic Polymorphic Ventricular Tachycardia Linked to the Triadin Gene. Journal of cardiovascular electrophysiology 26, 1146-1150.

Roston, T.M., Guo, W., Krahn, A.D., Wang, R., van Petegem, F., Sanatani, S., Chen, S.R.W., and Lehman, A. (2016). A novel RYR2 loss-of-function mutation (I4855M) is associated with left ventricular non-compaction and atypical catecholaminergic polymorphic ventricular tachycardia. Journal of electrocardiology.

Rubin, L.L., and Haston, K.M. (2011). Stem cell biology and drug discovery. BMC biology 9, 42.

Salama, G., and London, B. (2007). Mouse models of long QT syndrome. The Journal of Physiology 578, 43-53.

Satin, J., Itzhaki, I., Rapoport, S., Schroder, E.A., Izu, L., Arbel, G., Beyar, R., Balke, C.W., Schiller, J., and Gepstein, L. (2008). Calcium handling in human embryonic stem cellderived cardiomyocytes. Stem cells (Dayton, Ohio) 26, 1961-1972.

Sedej, S., Heinzel, F.R., Walther, S., Dybkova, N., Wakula, P., Groborz, J., Gronau, P., Maier, L.S., Vos, M.A., and Lai, F.A., et al. (2010). Na+-dependent SR Ca ${ }^{2+}$ overload induces arrhythmogenic events in mouse cardiomyocytes with a human CPVT mutation. Cardiovascular Research 87, 50-59.

Shan, J., Betzenhauser, M.J., Kushnir, A., Reiken, S., Meli, A.C., Wronska, A., Dura, M., Chen, B.-X., and Marks, A.R. (2010). Role of chronic ryanodine receptor phosphorylation in heart failure and $\beta$-adrenergic receptor blockade in mice. J Clin Invest 120, 4375-4387.

Shan, J., Xie, W., Betzenhauser, M., Reiken, S., Chen, B.X., Wronska, A., and Marks, A.R. (2012). Calcium leak through ryanodine receptors leads to atrial fibrillation in 3 mouse models of catecholaminergic polymorphic ventricular tachycardia. Circulation research 111, 708-717. 
Shinkuma, S., Guo, Z., and Christiano, A.M. (2016). Site-specific genome editing for correction of induced pluripotent stem cells derived from dominant dystrophic epidermolysis bullosa. Proceedings of the National Academy of Sciences of the United States of America 113, 5676-5681.

Siu, C.W., Lee, Y.K., Ho, J.C., Lai, W.H., Chan, Y.C., Ng, K.M., Wong, L.Y., Au, K.W., Lau, Y.M., and Zhang, J., et al. (2012). Modeling of lamin A/C mutation premature cardiac aging using patient-specific induced pluripotent stem cells. Aging 4, 803-822.

Smith, J., Bibikova, M., Whitby, F.G., Reddy, A.R., Chandrasegaran, S., and Carroll, D. (2000). Requirements for double-strand cleavage by chimeric restriction enzymes with zinc finger DNA-recognition domains. Nucleic acids research 28, 3361-3369.

Sommer, C.A., Stadtfeld, M., Murphy, G.J., Hochedlinger, K., Kotton, D.N., and Mostoslavsky, G. (2009). Induced pluripotent stem cell generation using a single lentiviral stem cell cassette. Stem cells (Dayton, Ohio) 27, 543-549.

Song, J., Yang, D., Xu, J., Zhu, T., Chen, Y.E., and Zhang, J. (2016). RS-1 enhances CRISPR/Cas9- and TALEN-mediated knock-in efficiency. Nature Communications 7, 10548.

Song, J., Zhong, J., Guo, X., Chen, Y., Zou, Q., Huang, J., Li, X., Zhang, Q., Jiang, Z., and Tang, C., et al. (2013). Generation of RAG 1- and 2-deficient rabbits by embryo microinjection of TALENs. Cell research 23, 1059-1062.

Soong, P.L., Tiburcy, M., and Zimmermann, W.H. (2012). Cardiac differentiation of human embryonic stem cells and their assembly into engineered heart muscle. Current protocols in cell biology / editorial board, Juan S. Bonifacino et al. Chapter 23, 8.

Stadtfeld, M., Nagaya, M., Utikal, J., Weir, G., and Hochedlinger, K. (2008). Induced pluripotent stem cells generated without viral integration. Science (New York, N.Y.) 322, 945-949.

Stover, A.E., and Schwartz, P.H. (2011). Adaptation of human pluripotent stem cells to feeder-free conditions in chemically defined medium with enzymatic single-cell passaging. Methods in molecular biology (Clifton, N.J.) 767, 137-146.

Streckfuss-Bomeke, K., Wolf, F., Azizian, A., Stauske, M., Tiburcy, M., Wagner, S., Hubscher, D., Dressel, R., Chen, S., and Jende, J., et al. (2013). Comparative study of human-induced pluripotent stem cells derived from bone marrow cells, hair keratinocytes, and skin fibroblasts. European Heart Journal 34, 2618-2629.

Studer, L., Vera, E., and Cornacchia, D. (2015). Programming and Reprogramming Cellular Age in the Era of Induced Pluripotency. Cell stem cell 16, 591-600.

Suetomi, T., Yano, M., Uchinoumi, H., Fukuda, M., Hino, A., Ono, M., Xu, X., Tateishi, H., Okuda, S., and Doi, M., et al. (2011). Mutation-linked defective interdomain interactions within ryanodine receptor cause aberrant $\mathrm{Ca}^{2+}$ release leading to catecholaminergic polymorphic ventricular tachycardia. Circulation 124, 682-694.

Sun, N., Yazawa, M., Liu, J., Han, L., Sanchez-Freire, V., Abilez, O.J., Navarrete, E.G., Hu, S., Wang, L., and Lee, A., et al. (2012). Patient-Specific Induced Pluripotent Stem Cells as a Model for Familial Dilated Cardiomyopathy. Science Translational Medicine 4, 130.

Sung, Y.H., Baek, I.J., Kim, D.H., Jeon, J., Lee, J., Lee, K., Jeong, D., Kim, J.S., and Lee, H.W. (2013). Knockout mice created by TALEN-mediated gene targeting. Nature biotechnology 31, 23-24. 
Sy, R.W., Gollob, M.H., Klein, G.J., Yee, R., Skanes, A.C., Gula, L.J., Leong-Sit, P., Gow, R.M., Green, M.S., and Birnie, D.H., et al. (2011). Arrhythmia characterization and long-term outcomes in catecholaminergic polymorphic ventricular tachycardia. Heart rhythm : the official journal of the Heart Rhythm Society 8, 864-871.

Takahashi, K., Tanabe, K., Ohnuki, M., Narita, M., Ichisaka, T., Tomoda, K., and Yamanaka, S. (2007). Induction of pluripotent stem cells from adult human fibroblasts by defined factors. Cell 131, 861-872.

Takahashi, K., and Yamanaka, S. (2006). Induction of pluripotent stem cells from mouse embryonic and adult fibroblast cultures by defined factors. Cell 126, 663-676.

Takeshima, H., Nishimura, S., Matsumoto, T., Ishida, H., Kangawa, K., Minamino, N., Matsuo, H., Ueda, M., Hanaoka, M., and Hirose, T., et al. (1989). Primary structure and expression from complementary DNA of skeletal muscle ryanodine receptor. Nature 339, 439-445.

Tateishi, H., Yano, M., Mochizuki, M., Suetomi, T., Ono, M., Xu, X., Uchinoumi, H., Okuda, S., Oda, T., and Kobayashi, S., et al. (2009). Defective domain-domain interactions within the ryanodine receptor as a critical cause of diastolic $\mathrm{Ca}^{2+}$ leak in failing hearts. Cardiovascular Research 81, 536-545.

Tesson, L., Usal, C., Menoret, S., Leung, E., Niles, B.J., Remy, S., Santiago, Y., Vincent, A.I., Meng, X., and Zhang, L., et al. (2011). Knockout rats generated by embryo microinjection of TALENs. Nature biotechnology 29, 695-696.

Tohyama, S., Hattori, F., Sano, M., Hishiki, T., Nagahata, Y., Matsuura, T., Hashimoto, H., Suzuki, T., Yamashita, H., and Satoh, Y., et al. (2013). Distinct metabolic flow enables large-scale purification of mouse and human pluripotent stem cell-derived cardiomyocytes. Cell stem cell 12, 127-137.

Uosaki, H., Fukushima, H., Takeuchi, A., Matsuoka, S., Nakatsuji, N., Yamanaka, S., and Yamashita, J.K. (2011). Efficient and scalable purification of cardiomyocytes from human embryonic and induced pluripotent stem cells by VCAM1 surface expression. PloS one 6, e23657.

Urbach, A., and Benvenisty, N. (2009). Studying Early Lethality of 45, XO (Turner's Syndrome) Embryos Using Human Embryonic Stem Cells. PLOS ONE 4, e4175.

Urbach, A., Schuldiner, M., and Benvenisty, N. (2004). Modeling for Lesch-Nyhan disease by gene targeting in human embryonic stem cells. Stem cells (Dayton, Ohio) 22, 635-641.

Valamehr, B., Abujarour, R., Robinson, M., Le T, Robbins, D., Shoemaker, D., and Flynn, P. (2012). A novel platform to enable the high-throughput derivation and characterization of feeder-free human iPSCs. Scientific reports 2.

van den Berg, C.W., Okawa, S., Chuva de Sousa Lopes, S.M., van Iperen, L., Passier, R., Braam, S.R., Tertoolen, L.G., del Sol, A., Davis, R.P., and Mummery, C.L. (2015). Transcriptome of human foetal heart compared with cardiomyocytes from pluripotent stem cells. Development (Cambridge, England) 142, 3231-3238.

van der Oost, J., Westra, E.R., Jackson, R.N., and Wiedenheft, B. (2014). Unravelling the structural and mechanistic basis of CRISPR-Cas systems. Nature reviews. Microbiology 12, 479-492. 
van der Werf, C., Kannankeril, P.J., Sacher, F., Krahn, A.D., Viskin, S., Leenhardt, A., Shimizu, W., Sumitomo, N., Fish, F.A., and Bhuiyan, Z.A., et al. (2011). Flecainide Therapy Reduces Exercise-Induced Ventricular Arrhythmias in Patients With Catecholaminergic Polymorphic Ventricular Tachycardia. Journal of the American College of Cardiology 57, 2244-2254.

van der Werf, C., Zwinderman, A.H., and Wilde, A.A. (2012). Therapeutic approach for patients with catecholaminergic polymorphic ventricular tachycardia: state of the art and future developments. Europace: European pacing, arrhythmias, and cardiac electrophysiology: journal of the working groups on cardiac pacing, arrhythmias, and cardiac cellular electrophysiology of the European Society of Cardiology 14, 175-183.

van Hoof, D., Dormeyer, W., Braam, SR, Passier, R., Monshouwer-Kloots, J., Ward-van, O.D., Heck, A.J., Krijgsveld, J., and Mummery, C.L. (2010). Identification of cell surface proteins for antibody-based selection of human embryonic stem cell-derived cardiomyocytes. Journal of proteome research 9, 1610-1618.

Vasquez, K.M., Marburger, K., Intody, Z., and Wilson, J.H. (2001). Manipulating the mammalian genome by homologous recombination. PNAS 98, 8403-8410.

Wang, X., Sun, Q., McGrath, S.D., Mardis, E.R., Soloway, P.D., and Clark, A.G. (2008). Transcriptome-wide identification of novel imprinted genes in neonatal mouse brain. PloS one 3 , e3839.

Warren, L., Manos, P.D., Ahfeldt, T., Loh, Y.H., Li, H., Lau, F., Ebina, W., Mandal, P.K., Smith, Z.D., and Meissner, A., et al. (2010). Highly efficient reprogramming to pluripotency and directed differentiation of human cells with synthetic modified mRNA. Cell stem cell 7, 618-630.

Watanabe, H., Chopra, N., Laver, D., Hwang, H.S., Davies, S.S., Roach, D.E., Duff, H.J., Roden, D.M., Wilde, A.A., and Knollmann, B.C. (2009). Flecainide prevents catecholaminergic polymorphic ventricular tachycardia in mice and humans. Nature medicine 15, 380-383.

Wehrens, X.H., Lehnart, S.E., Reiken, S., van der Nagel, R., Morales, R., Sun, J., Cheng, Z., Deng, S.X., Windt, L.J. de, and Landry, D.W., et al. (2005). Enhancing calstabin binding to ryanodine receptors improves cardiac and skeletal muscle function in heart failure. Proceedings of the National Academy of Sciences of the United States of America 102, 9607-9612.

Wehrens, X.H.T., Lehnart, S.E., Reiken, S., Vest, J.A., Wronska, A., and Marks, A.R. (2006). Ryanodine receptor/calcium release channel PKA phosphorylation: a critical mediator of heart failure progression. Proceedings of the National Academy of Sciences of the United States of America 103, 511-518.

Wehrens, X.H.T., Lehnart, S.E., Reiken, S.R., Deng, S.-X., Vest, J.A., Cervantes, D., Coromilas, J., Landry, D.W., and Marks, A.R. (2004a). Protection from Cardiac Arrhythmia Through Ryanodine Receptor-Stabilizing Protein Calstabin2. Science 304, 292-296.

Wehrens, X.H.T., Lehnart, S.E., Reiken, S.R., and Marks, A.R. (2004b). Ca ${ }^{2+} / \mathrm{calmodulin}^{-}$ dependent protein kinase II phosphorylation regulates the cardiac ryanodine receptor. Circulation research 94, e61-70. 
Willems, E., Cabral-Teixeira, J., Schade, D., Cai, W., Reeves, P., Bushway, P.J., Lanier, M., Walsh, C., Kirchhausen, T., and Izpisua, B.J.C., et al. (2012). Small molecule-mediated TGFbeta type II receptor degradation promotes cardiomyogenesis in embryonic stem cells. Cell stem cell 11, 242-252.

Witcher, Kovacs, R.J., Schulman, H., Cefali, D.C., and Jones, L.R. (1991). Unique phosphorylation site on the cardiac ryanodine receptor regulates calcium channel activity. The Journal of biological chemistry 266, 11144-11152.

www.neb.com. https://www.neb.com/tools-and-resources/feature-articles/crispr-cas9and-targeted-genome-editing-a-new-era-in-molecular-biology.

Xiao, B., Jiang, M.T., Zhao, M., Yang, D., Sutherland, C., Lai, F.A., Walsh, M.P., Warltier, D.C., Cheng, H., and Chen, S.R.W. (2005). Characterization of a novel PKA phosphorylation site, serine-2030, reveals no PKA hyperphosphorylation of the cardiac ryanodine receptor in canine heart failure. Circulation research 96, 847-855.

Xiao, B., Zhong, G., Obayashi, M., Yang, D., Chen, K., Walsh, M.P., Shimoni, Y., Cheng, H., Ter Keurs, H., and Chen, S.R.W. (2006). Ser-2030, but not Ser-2808, is the major phosphorylation site in cardiac ryanodine receptors responding to protein kinase $A$ activation upon $\beta$-adrenergic stimulation in normal and failing hearts. Biochemical Journal 396, 7-16.

Xiao, R.-P. (2001). $\beta$-Adrenergic Signaling in the Heart: Dual Coupling of the $\beta 2$-Adrenergic Receptor to Gs and Gi Proteins. Sci. STKE 2001, re15-re15.

Xu, C., Police, S., Rao, N., and Carpenter, M.K. (2002). Characterization and enrichment of cardiomyocytes derived from human embryonic stem cells. Circulation research 91, 501508.

Yang, H., Wang, H., Shivalila, C.S., Cheng, A.W., Shi, L., and Jaenisch, R. (2013). One-step generation of mice carrying reporter and conditional alleles by CRISPR/Cas-mediated genome engineering. Cell 154, 1370-1379.

Yang, X., Rodriguez, M., Pabon, L., Fischer, K.A., Reinecke, H., Regnier, M., Sniadecki, N.J., Ruohola-Baker, H., and Murry, C.E. (2014). Tri-iodo-I-thyronine promotes the maturation of human cardiomyocytes-derived from induced pluripotent stem cells. Journal of molecular and cellular cardiology 72, 296-304.

Yano, M., Kobayashi, S., Kohno, M., Doi, M., Tokuhisa, T., Okuda, S., Suetsugu, M., Hisaoka, T., Obayashi, M., and Ohkusa, T., et al. (2003). FKBP12.6-Mediated Stabilization of Calcium-Release Channel (Ryanodine Receptor) as a Novel Therapeutic Strategy Against Heart Failure. Circulation 107, 477-484.

Yazawa, M., Hsueh, B., Jia, X., Am Pasca, Bernstein, J.A., Hallmayer, J., and Dolmetsch, R.E. (2011). Using induced pluripotent stem cells to investigate cardiac phenotypes in Timothy syndrome. Nature 471, 230-234.

Yu, C., Liu, Y., Ma, T., Liu, K., Xu, S., Zhang, Y., Liu, H., La Russa, M., Xie, M., and Ding, S., et al. (2015). Small molecules enhance CRISPR genome editing in pluripotent stem cells. Cell stem cell 16, 142-147.

Yu, J., Vodyanik, M.A., Smuga-Otto, K., Antosiewicz-Bourget, J., Frane, J.L., Tian, S., Nie, J., Jonsdottir, G.A., Ruotti, V., and Stewart, R., et al. (2007). Induced Pluripotent Stem Cell Lines Derived from Human Somatic Cells. Science 318, 1917-1920. 
Yuchi, Z., Lau, K., and van Petegem, F. (2012). Disease mutations in the ryanodine receptor central region: crystal structures of a phosphorylation hot spot domain. Structure (London, England: 1993) 20, 1201-1211.

Zaragoza, C., Gomez-Guerrero, C., Martin-Ventura, J.L., Blanco-Colio, L., Lavin, B., Mallavia, B., Tarin, C., Mas, S., Ortiz, A., and Egido, J. (2011). Animal models of cardiovascular diseases. Journal of biomedicine \& biotechnology 2011, 497841.

Zhabyeyev, P., Hiess, F., Wang, R., Liu, Y., Wayne Chen, S.R., and Oudit, G.Y. (2013). S4153R is a gain-of-function mutation in the cardiac $\mathrm{Ca}^{(2+)}$ release channel ryanodine receptor associated with catecholaminergic polymorphic ventricular tachycardia and paroxysmal atrial fibrillation. The Canadian journal of cardiology 29, 993-996.

Zhang, J., Nuebel, E., Daley, G.Q., Koehler, C.M., and Teitell, M.A. (2012a). Metabolic regulation in pluripotent stem cells during reprogramming and self-renewal. Cell stem cell 11, 589-595.

Zhang, Q., Jiang, J., Han, P., Yuan, Q., Zhang, J., Zhang, X., Xu, Y., Cao, H., Meng, Q., and Chen, L., et al. (2011). Direct differentiation of atrial and ventricular myocytes from human embryonic stem cells by alternating retinoid signals. Cell research $21,579-587$.

Zhang, W.Y., Almeida, P.E.d. and Wu, J.C. (2012b). Teratoma formation: A tool for monitoring pluripotency in stem cell research (Harvard Stem Cell Institute).

Zhang, X.-H., Haviland, S., Wei, H., Saric, T., Fatima, A., Hescheler, J., Cleemann, L., and Morad, M. (2013). Ca2+ signaling in human induced pluripotent stem cell-derived cardiomyocytes (iPS-CM) from normal and catecholaminergic polymorphic ventricular tachycardia (CPVT)-afflicted subjects. Cell calcium 54, 57-70.

Zhang, X.-H., Wei, H., Saric, T., Hescheler, J., Cleemann, L., and Morad, M. (2015). Regionally diverse mitochondrial calcium signaling regulates spontaneous pacing in developing cardiomyocytes. Cell calcium 57, 321-336.

Zhao, Y.T., Valdivia, C.R., Gurrola, G.B., Powers, P.P., Willis, B.C., Moss, R.L., Jalife, J., and Valdivia, H.H. (2015). Arrhythmogenesis in a catecholaminergic polymorphic ventricular tachycardia mutation that depresses ryanodine receptor function. Proceedings of the National Academy of Sciences of the United States of America 112, 77.

Zhu, W.-Z., Xie, Y., Moyes, K.W., Gold, J.D., Askari, B., and Laflamme, M.A. (2010). Neuregulin/ErbB signaling regulates cardiac subtype specification in differentiating human embryonic stem cells. Circulation research 107, 776-786.

Zimmermann, W.H., Schneiderbanger, K., Schubert, P., Didie, M., Munzel, F., Heubach, J.F., Kostin, S., Neuhuber, W.L., and Eschenhagen, T. (2002). Tissue engineering of a differentiated cardiac muscle construct. Circulation research 90, 223-230.

Zou, Y., Liang, Y., Gong, H., Zhou, N., Ma, H., Guan, A., Sun, A., Wang, P., Niu, Y., and Jiang, H., et al. (2011). Ryanodine Receptor Type 2 Is Required for the Development of Pressure Overload-Induced Cardiac Hypertrophy. Hypertension 58, 1099-1110. 


\section{Acknowledgements}

I would like to take the opportunity to express my deepest gratitude to all people who supported me during my doctoral studies over the last years.

First, I especially thank Prof. Dr. Kaeomei Guan for giving me the opportunity to be a doctoral student in her laboratory. I am grateful for her supervision, her excellent support of my work and her encouragement in difficult situations. I would like to thank her for giving me the freedom to develop my own ideas and for continuously being open to my questions. Moreover, I am grateful that she proof-reads my thesis despite her busy schedule and the large distance to Dresden.

I sincerely thank my second referee Prof. Dr. Martin Oppermann for his helpful scientific discussions during the thesis committee meetings.

I am grateful to Prof. Dr. Walter Stühmer for his guidance and helpful scientific suggestions during my PhD study.

I also extend my sincere thanks to the other members of my examination board: Prof. Dr. Ralf Dressel, Prof. Dr. mult. Thomas Meyer and Dr. Katrin Streckfuß-Bömeke for taking time to serve as my examiners and for evaluating my thesis despite having to handle many other tasks.

A big thanks goes to Dr. Lukas Cyganek for your helpful enthusiastic support, the great teamwork and the friendly relationship during your time as Postdoc in Guan's lab.

I would like to thank my former colleagues Wener Li and Xiaojing Luo for their nice cooperative work, especially sharing their cardiomyocytes with me, in the CPVT project. Moreover, I thank Karolina Sekeres for her time for helping me with the calcium measurements and analysis in the CRISPR/Cas9 project.

Thanks go to Dr. Gabriela Salinas and Dr. Thomas Lingner from the Transcriptome Analysis Laboratory Göttingen for the fast results and analysis of the allele-specific sequencing.

I am grateful to all my friends as well as current and former colleagues of the Department of Cardiology and Pneumology (third and fourth floor!) for their support and the friendly working atmosphere. I thank you all for your cooperation and the feeding of my cells during the weekend service. I will also not forget our entertaining lab outings, the funny morning breakfast and lunch times together. A really big thanks goes to Johanna Heine for your great support in the last year of my thesis as well as Marius Klangwart, and Simin Chen for their helpful assistance in the CPVT project.

Last but not least I want to thank my parents and family for your unconditionally love, moral support, total confidence and for always believing in me. You always encouraged me, especially during the difficult periods of my PhD thesis. I thank Patrick for his love, care, and support and to make me laugh in stressful situations. I am so happy that I met you in Costa Rica (:). 


\section{Appendix}

\subsection{Abbreviations}

\begin{tabular}{|c|c|}
\hline${ }^{\circ} \mathrm{C}$ & Degree celsius \\
\hline$\mu$ & Micro \\
\hline$\mu \mathrm{g}$ & Microgram \\
\hline a.u. & Arbitrary units \\
\hline aа & Amino acids \\
\hline AFP & Alpha-1-fetoprotein \\
\hline ALB & Albumin \\
\hline APD & Action potential duration \\
\hline Approx. & Approximately \\
\hline APS & Ammonium persulfate \\
\hline ATP & Adenosine triphosphate \\
\hline ASE & Allele-specific expression \\
\hline BafA1 & Bafilomycin A1 \\
\hline bp & Base pair \\
\hline BSA & Bovine serum albumin \\
\hline $\mathrm{Ca}^{2+}$ & Calcium \\
\hline CaM & Calmodulin \\
\hline CamKII & $\mathrm{Ca}^{2+} /$ Calmodulin-dependent protein kinase II \\
\hline CAMP & Cyclic adenosine monophosphate \\
\hline Cas9 & CRISPR-associated protein 9 \\
\hline CASQ2 & Calsequestrin 2 \\
\hline cDNA & Complementary DNA \\
\hline $\mathrm{CM}$ & Cardiomyocyte \\
\hline c-MYC & V-myc myelocytomatosis avian viral oncogene homolog \\
\hline $\mathrm{CO}_{2}$ & Carbon dioxide \\
\hline CPVT & Catecholaminergic polymorphic ventricular tachycardia \\
\hline CRISPR & Clustered regularly interspaced short palindromic repeats \\
\hline cTNT & Cardiac troponin $\mathrm{T}$ \\
\hline $\mathrm{Cx} 43$ & Connexin 43 \\
\hline DAD & Delayed afterdepolarization \\
\hline DAPI & 4', 6-Diamidino-2-phenylindole dihydrochloride \\
\hline $\mathrm{dd}_{2} \mathrm{O}$ & Double distilled water \\
\hline DMEM & Dulbecco's modified eagle medium \\
\hline
\end{tabular}




\begin{tabular}{|c|c|}
\hline DMSO & Dimethylsulfoxide \\
\hline DNA & Deoxyribonucleic Acid \\
\hline DPBS & Dulbecco's phosphate buffered saline \\
\hline DSB & Double strand break \\
\hline DTT & Dithiothreitol \\
\hline E. coli & Escherichia coli \\
\hline EAD & Early afterdepolarization \\
\hline $\mathrm{ECL}$ & Enhanced chemiluminescence \\
\hline EDTA & Ethylene diamine tetra acetic acid \\
\hline ESCS & Embryonic stem cells \\
\hline $\mathrm{F}$ & Fluorescence \\
\hline FACS & Fluorescence-activated cell sorting \\
\hline FBS & Fetal bovine serum \\
\hline FDHM & Full duration at half maximum \\
\hline Fig. & Figure \\
\hline FITC & Fluorescein isothiocyanate \\
\hline FL & Feeder layer \\
\hline for & Forward \\
\hline FOXD3 & Forkhead box D3 \\
\hline FSC & Forward scatter \\
\hline FWHM & Full width at half maximum \\
\hline g & Gram \\
\hline GAPDH & Glyceraldehyde 3-phosphate dehydrogenase \\
\hline GDF3 & Growth differentiation factor 3 \\
\hline gDNA & Genomic DNA \\
\hline $\mathrm{h}$ & Hours \\
\hline hbFGF & Fibroblast growth factor basic \\
\hline HDR & Homology directed repair \\
\hline HEK293 & Human embryonic kidney 293 \\
\hline HEPES & 4-(2-Hydroxyethyl)-1-piperazineethanesulfonic acid \\
\hline hERG & Human ether-à-go-go-related gene \\
\hline hESC & Human embryonic stem cell \\
\hline hiPSC & Human induced pluripotent stem cell \\
\hline $\mathrm{HR}$ & Homologous recombination \\
\hline HRP & Horseradish peroxidase \\
\hline $\mathrm{Hz}$ & Hertz \\
\hline IP3R & Inositol 1,4,5-trisphosphate receptors \\
\hline
\end{tabular}




\begin{tabular}{|c|c|}
\hline IRX4 & Iroquois homeobox 4 \\
\hline Iso & Isoprenaline \\
\hline $\mathrm{KCl}$ & Potassium chloride \\
\hline kDa & Kilodalton \\
\hline KIR2.1 & Inward rectifier potassium channel 2 \\
\hline KLF4 & Krüppel-like factor 4 \\
\hline I & Liter \\
\hline $\mathrm{mA}$ & Milliampere \\
\hline MEA & Multi-electrode array \\
\hline MEFs & Mouse embryonic fibroblasts \\
\hline $\min$ & Minutes \\
\hline $\mathrm{ml}$ & Milliliter \\
\hline MLC2A & Myosin light chain $2 a$ \\
\hline MLC2V & Myosin light chain $2 \mathrm{v}$ \\
\hline MOI & Multiplicity of infection \\
\hline ms & Milliseconds \\
\hline MSC & Mesenchymal stem cell \\
\hline MTG & Monothioglycerol \\
\hline $\mathrm{n}$ & Nano \\
\hline $\mathrm{Na}_{2} \mathrm{HPO}_{4}$ & Disodium hydrogen phosphate \\
\hline $\mathrm{NaOH}$ & Sodium hydroxide \\
\hline Nav1.5 & Cardiac voltage-gated $\mathrm{Na}^{+}$channel \\
\hline NCX & Sodium-calcium exchanger \\
\hline NEAA & Non-essential amino acids \\
\hline OCT4 & Octamer binding transcription factor 4 \\
\hline OCT4 & Octamer-binding transcription factor 4 \\
\hline PAGE & Polyacrylamide gel electrophoresis \\
\hline PCR & Polymerase chain reaction \\
\hline PDE4D3 & Phosphodiesterase 4D3 \\
\hline PE & Phycoerythrin \\
\hline $\mathrm{pF}$ & Picofarad \\
\hline PFA & Paraformaldehyde \\
\hline $\mathrm{pH}$ & Negative logarithmic value of the $\mathrm{H}^{+}$concentration \\
\hline PLB & Phospholamban \\
\hline PP1 & Protein phosphatase 1 \\
\hline PP2A & Protein phosphatase $2 a$ \\
\hline PTC & Premature termination codon \\
\hline
\end{tabular}




\begin{tabular}{|c|c|}
\hline rev & Reverse \\
\hline rpm & Revolutions per minute \\
\hline RPMI & Roswell Park Memorial Institute \\
\hline RT & Room temperature \\
\hline RYR & Ryanodine receptor \\
\hline SDS & Sodium sodecyl sulfate \\
\hline sec & Seconds \\
\hline SEM & Standard error \\
\hline Ser & Serine \\
\hline SERCA & Sacro/ endoplasmic reticulum $\mathrm{Ca}^{2+-}$ ATPase \\
\hline $\mathrm{SeV}$ & Sendai virus \\
\hline SMA & Smooth muscle actin \\
\hline SOX2 & Sex determining region $\mathrm{Y}$ box 2 \\
\hline Sox2 & Sex Determining Region Y-Box 2 \\
\hline SR & Sacroplasmic reticulum \\
\hline SSEA4 & Stage-specific embryonic antigen- 4 \\
\hline ssODNs & Single-stranded oligodeoxynucleotides \\
\hline STEMCCA & Stem cell cassette \\
\hline SYP & Synaptophysin \\
\hline Tab. & Table \\
\hline TALEN & Transcription activator-like nuclease \\
\hline TBE buffer & Tris-borate EDTA buffer \\
\hline TBS & Tris buffered saline \\
\hline TBS-T & Tris buffered saline with Tween-20 \\
\hline TC & Tissue culture \\
\hline TEMED & Tetramethylenediamine \\
\hline $\mathrm{TH}$ & Tyrosine hydroxylase \\
\hline Thr & Threonine \\
\hline TRA-1-60 & Tumor rejection antigen 1-60 \\
\hline Tris & Tris-(hydroxymethyl)-aminomethane \\
\hline TZV & Thiazovivin \\
\hline U & Unit \\
\hline V & Voltage \\
\hline$v$ & Volume \\
\hline VCAM-1 & Vascular cell adhesion molecule 1 \\
\hline VT & Ventricular tachycardia \\
\hline ZFN & Zinc finger nuclease \\
\hline
\end{tabular}


$\alpha$

$\alpha-\mathrm{ACT}$

$\alpha-\mathrm{MHC}$

$\beta$

Alpha

$\alpha$-Actinin

Myosin heavy chain $\alpha$ isoform

Beta 


\subsection{List of Figures}

Figure 1. $\mathrm{Ca}^{2+}$-induced $\mathrm{Ca}^{2+}$ release and triggered arrhythmias....................................... 5

Figure 2. Structure of RYR2 including hot-spot regions of mutations. ................................ 6

Figure 3. Generation of patient-specific hiPSCs for disease modeling and drug screening. 9

Figure 4. Key steps sufficient for cardiac differentiation of hiPSCs. ................................... 16



Figure 6. CRISPR/Cas9 plasmid containing the sequence of gRNA, Cas9, GFP and

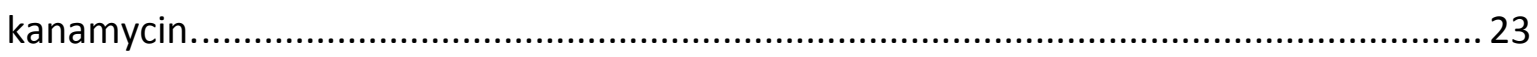

Figure 7. RYR2 protein and mutations studied in the present study.............................. 54

Figure 8. Timeline for the generation of CPVT-hiPSCs. .................................................... 55

Figure 9. Morphology and alkaline phosphatase activity of CPVT-hiPSCs. ........................ 55

Figure 10. Gene expression analysis of generated CPVT-hiPSCs and their parental fibroblasts.

Figure 11. Immunostaining detects pluripotency-related proteins of generated CPVThiPSCs.

Figure 12. Gene expression of differentiated CPVT-hiPSCs.

Figure 13. Immunostaining of differentiated hiPSCs detects germ layer-specific proteins.



Figure 15. Verification of the RYR2 point mutation in the generated CPVT-hiPSCs. ..........62 62

Figure 16. Schematic illustration of the directed cardiac differentiation using hiPSCs......63

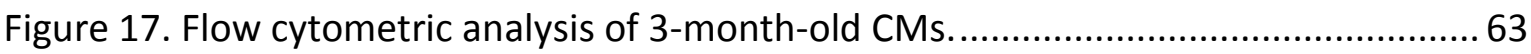



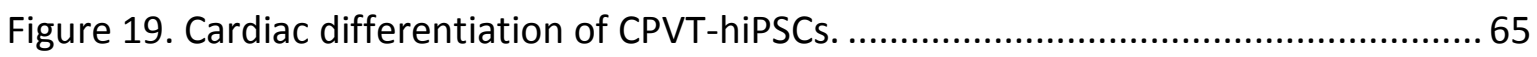

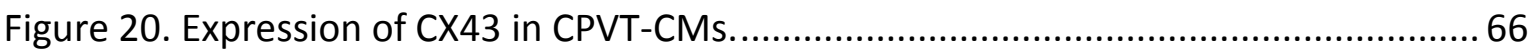



Figure 22. Basal and isoprenaline-induced $\mathrm{Ca}^{2+}$ sparks in CPVT- and Ctrl-CMs. ..................68

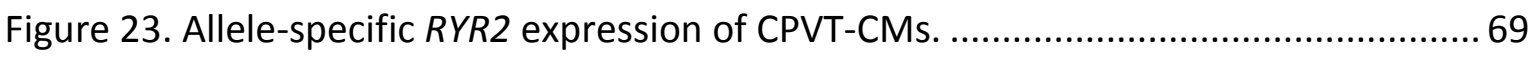

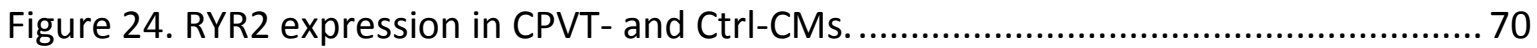

Figure 25. Phospho-RYR2 expression in CPVT- and Ctrl-CMs........................................... 71

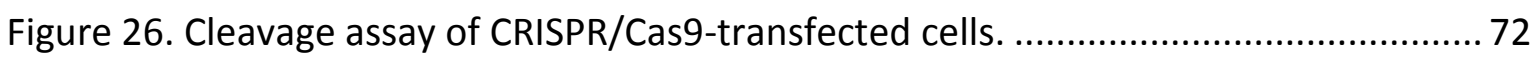

Figure 27. Strategy for the generation of CRISPR/Cas9-targeted hiPSCs......................... 73 
Figure 28. Screening of CRISPR/Cas9-transfected cell clones.......................................... 74

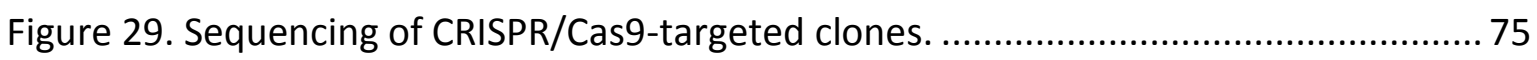

Figure 30. Gene expression of $\mathrm{Ca}^{2+}$ regulating genes in CRISPR/Cas9-edited CMs. ........... 76

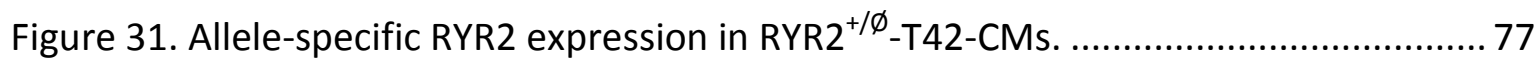

Figure 32. RYR2 expression in CRISPR/Cas9-edited CMs. ................................................ 78

Figure 33. Immunostaining of RYR2 ${ }^{\varnothing / \varnothing}-\mathrm{A} 3-\mathrm{CMs}$ in comparison to CPVTC2.1-CMs............. 79

Figure 34. Analysis of RYR2 protein degradation in CRISPR/Cas9-edited CMs.................. 80

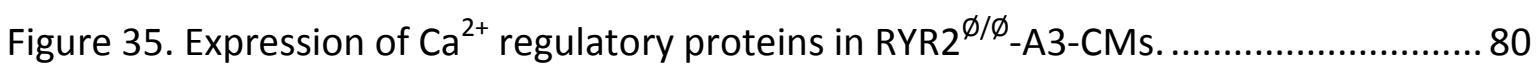

Figure 36. Isoprenaline-induced $\mathrm{Ca}^{2+}$ sparks in CRISPR/Cas9-engineered CMs.................. 81

Figure 37. $\mathrm{Ca}^{2+}$ transients from CRISPR/Cas9-engineered CMs. ...................................... 82 


\subsection{List of Tables}

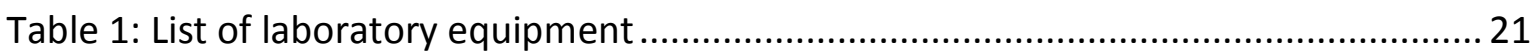

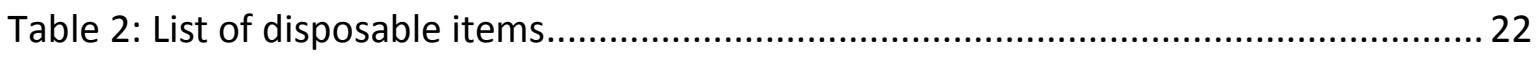

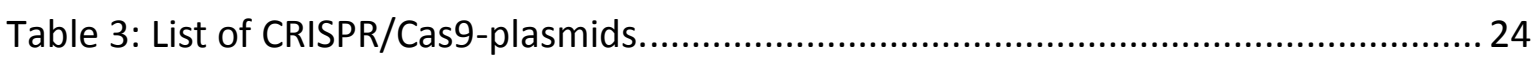

Table 4: List of oligonucleotides for reverse transcription-PCR analysis ........................... 24

Table 5: Single-stranded oligodesoxynucleotides (ssODNs) used for CRISPR/Cas9

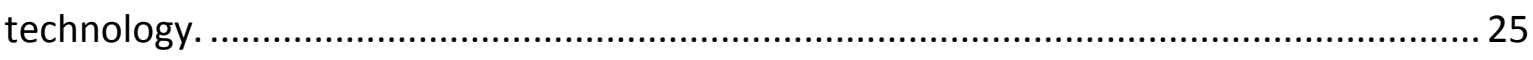

Table 6: List of oligonucleotides used for screening of CRISPR/Cas9-edited clones .......... 26

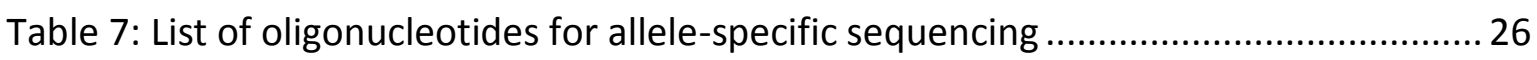



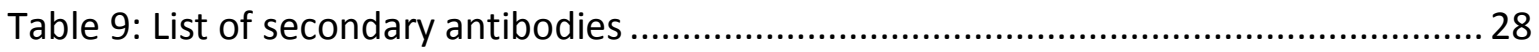

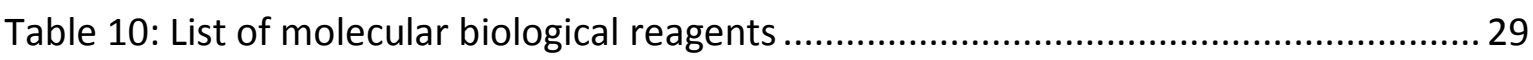

Table 11: List of components for molecular biological methods, and protein analyses .... 31

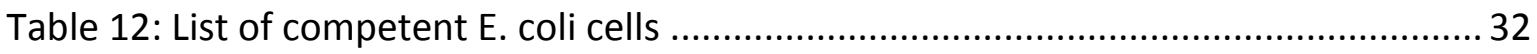

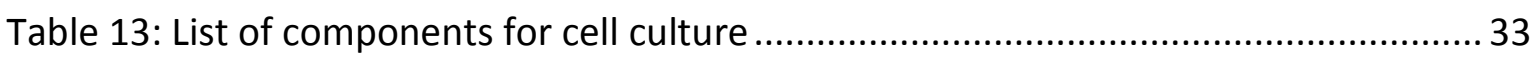





Table 16: hiPSC lines used in this work and their specifications...................................... 36

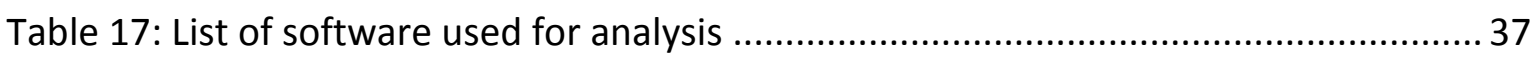

Table 18: List of components for reverse transcription reaction................................... 43

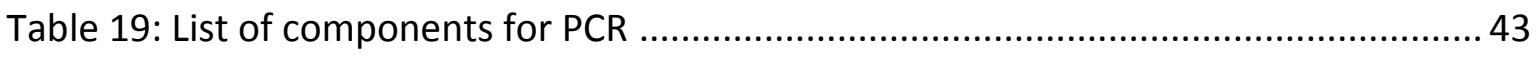

Table 20: PCR for the amplification of CDNA used for allele-specific expression analysis . 46

Table 21: Components for $12 \mathrm{ml}$ separation gel and $7.5 \mathrm{ml}$ stacking gel ......................... 47 


\subsection{Curriculum Vitae}

Personal details

\begin{tabular}{ll}
\hline Name & Sarah Henze \\
Date of birth & $06 / 11 / 1988$ \\
Place of birth & Göttingen, Germany \\
Nationality & German \\
Address & Falkenhorst 12, 34346 Hann. Münden
\end{tabular}

Education

Since Mai 2013 PhD study of Molecular Medicine at the Department of Cardiology and Pneumology, Georg-August University Göttingen

PhD Thesis: Induced pluripotent stem cell-derived cardiomyocytes as model for studying CPVT caused by mutations in RYR2

10/2010 - 03/2013 Master of Science study of "Developmental, Neural and Behavioral Biology" at the Georg-August University Göttingen

Master thesis: Chemical-versus Ligand-induced Heterodimerization of $\beta$-Arrestin and Chemokine Receptors CXCR4/CCR5

10/2007-10/2010 Bachelor of Science, Molecular Biosciences, at the Georg-August-University Göttingen

Bachelor thesis: "Identifizierung der Interaktionspartner der S-Adenosylmethioninsynthetase unter der Verwendung der Tandem-Affinitäts-Aufreinigung in Aspergillus nidulans"

2004-2007

"Berufliches Gymnasium", Specialization: Biotechnology, Witzenhausen

2000-2004

“Werra-Realschule”, Hann. Münden

1994-2000

Primary School "Königshof" and "Orientierungsstufe I", Hann. Münden 